Portland State University

PDXScholar

1986

\title{
A Multidimensional Analysis of Physical Attractiveness in the Formation of First Impressions
}

Jewel Marianna Smith Hunter

Portland State University

Follow this and additional works at: https://pdxscholar.library.pdx.edu/open_access_etds

Part of the Interpersonal and Small Group Communication Commons

Let us know how access to this document benefits you.

\section{Recommended Citation}

Smith Hunter, Jewel Marianna, "A Multidimensional Analysis of Physical Attractiveness in the Formation of First Impressions" (1986). Dissertations and Theses. Paper 3634.

https://doi.org/10.15760/etd.5502

This Thesis is brought to you for free and open access. It has been accepted for inclusion in Dissertations and Theses by an authorized administrator of PDXScholar. Please contact us if we can make this document more accessible: pdxscholar@pdx.edu. 
AN ABSTRACT OF THE THESIS of Jewel Mariannna Smith Hunter for the Master of Science in Speech Communication presented August 25, 1986.

Title: A Multidimensional Analysis of Physical Attractiveness in the Formation of First Impressions.

APPROVED BY MEMBERS OF THE THESIS COMMITTEE:

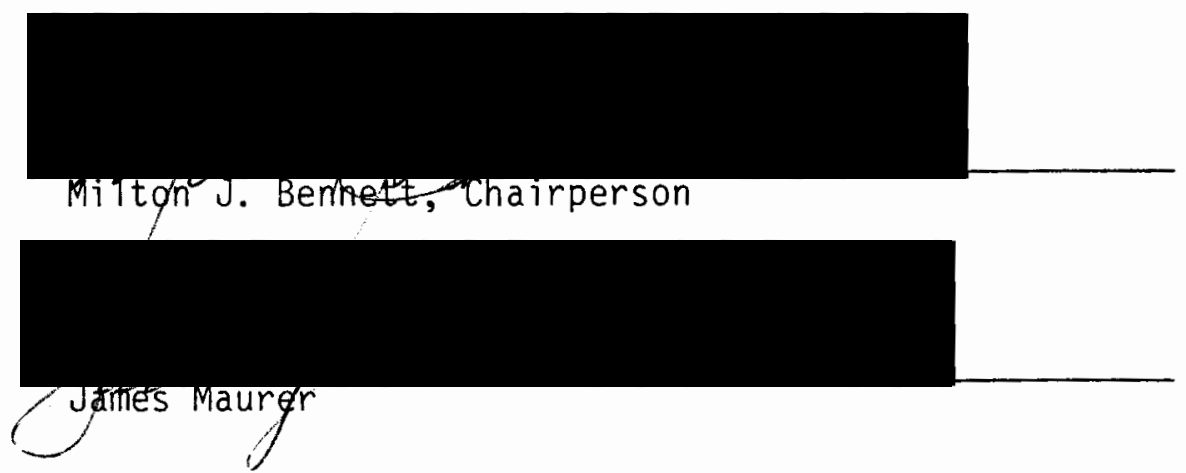

Before any words are spoken, an individual's appearance is his or her first line of nonverbal communication with the rest of the world. Prior research conducted on physical attractiveness has been vague and contradictory and has not assessed its many components. Rather, past investigators have perceived physical attractiveness as a "unidimensional" concept.

Several phases were involved in the task of proving or disproving the hypotheses of the study. The first general phase began with the selection of photographic subjects, progressed into the creation of a set of photographs which were to become the stimuli, and proceeded to the selection of the photo judges, whose evaluations 
would determine the final select group of 18 photographs and their physical attractiveness intensity levels.

Next, an initial questionnaire (OFIQ) was designed and constructed, then tested and retested to ascertain its inter-item reliability. The questionnaire differed from all its predecessors in physical attractiveness research because of its unique multidimensional approach. It was followed by a modified questionnaire (MFIQ) that resulted from statistical analyses of the original version.

The final phase in testing the three hypotheses involved 72 young male and female subjects between the ages of 19 to 24 who rated 18 stimulus photographs using the MFIQ to provide the necessary data for an in-depth statistical analysis of physical attractiveness across age-group and gender.

Statistical analyses used to examine the research hypotheses were descriptive statistics of mean age group comparisons, one-way t-test and analysis of variance on each of the five components defining physical attractiveness. Calculations were performed by a Honeywel1 66/40 computer using the implementation of Statistical Package for Social Sciences.

Hypothesis I asserted that Young Female stimulus photographs would be rated more positively in physical attractiveness than either the Middle-Aged or Older stimulus photographs by subjects in the 19 to 24 age group (young). Results indicated that both young male and female subjects rated Young Female stimulus photographs more 
positively in Physical Attributes and Instrumental Qualities. Young female subjects rated the same two components highly but considered Affect and Behavior Predisposition equally important. Physical Attributes and Instrumental Qualities of the Young Female stimulus photographs were associated with more positive ratings than the Middle-Aged or 01 der stimulus photographs.

The second hypothesis postulated that young subjects would evaluate the Middle-Aged Male as being more physically attractive than either the Young or 01 der Male stimulus photographs. Findings indicated that this age group was seen as less attractive in Physical Attributes but more attractive in Instrumental Qualities. Overall, the multidimensional method of physical attractiveness analysis disproved the hypothesis that Middle-Aged Males would be found more physically attractive than males in the Younger and 01 der groups.

Hypothesis III predicted that the more the person in the stimulus photograph was liked (indicated by a high score in Affect), the higher young subjects of either gender would rate the stimulus photograph in the other four components. Hypothesis III indicated a significant relationship between Affect and the other four components of physical attractiveness. This finding supports the investigator's perspective of examining physical attractiveness multidimensionally rather than as a "unidimensional" concept. 
A MULTIDIMENSIONAL ANALYSIS OF

PHYSICAL ATTRACTIVENESS IN THE

FORMATION OF FIRST IMPRESSIONS

By

Jewel Marianna Smith Hunter

A thesis submitted in partial fulfillment

of the requirements for the degree of

MASTER OF SCIENCE

in

SPEECH COMMUNICATION

Portland State University

1986 
TO THE OFFICE OF GRADUATE STUDIES AND RESEARCH:

The members of the Committee approve the thes is of Jewe1 Marianna Smith Hunter presented on August 25, 1986.

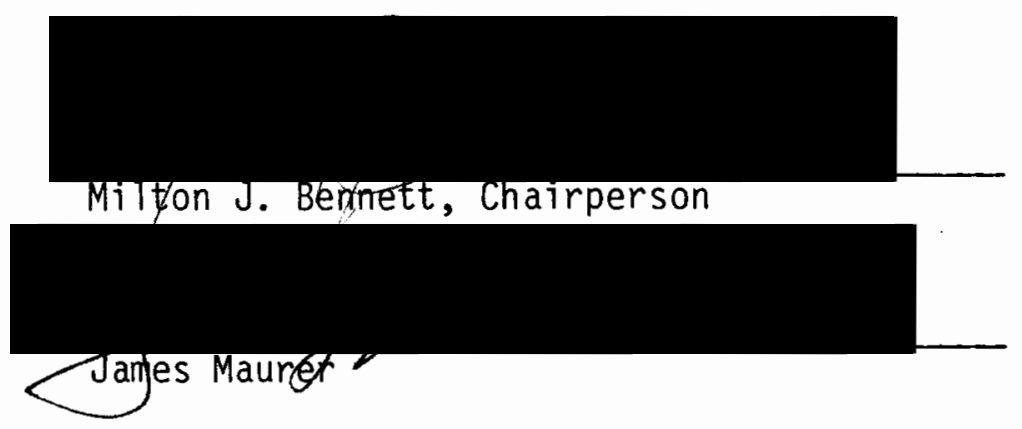

APPROVED:

Theodore G. Grove, Chairperson, Department of Speech Communication Bernard Ross, Acting Dean of Graduate Studies and Research 


\section{ACKNOWLEDGEMENTS}

Acknowiedgements are due many people who have helped to make this study possible as participants, committee members, consultants, family and friends.

First and foremost, I am grateful to Milton Bennett, the chairperson of my committee, for being there when I needed him, for his advice and his help in editing my manuscript.

I appreciated the assistance of Theodore Grove, Head, Speech Communication Department, in providing computer funds and discussing the statistical design of the study.

James Maurer of the Speech Pathology Department and Earle MacCannell of the Sociology Department offered valuable insights during the development of this thesis.

The Institute on Aging at Portland State University helped me to develop my potential as a researcher more fully by employing me and exposing me to a multitude of on-going research projects prior to the beginning of my thesis.

For those too numerous to mention, I thank all those people who participated in this study. Without their help, this study would never have been possible.

I would like to thank all my friends for being there when I needed them to provide a good ear, encouragement and "welcomed 
divertisements". Sandy Carter, I appreciated your help in proofing and editing my "albatross". Marilyn Petersen, thank you for the time you gave me to discuss my study.

I dedicate this thesis to my father and mother, Robert and Faith Smith, whose love, encouragement, emotional and financial support have helped me to achieve the completion of this study and my degrees. 
TABLE OF CONTENTS

PAGE

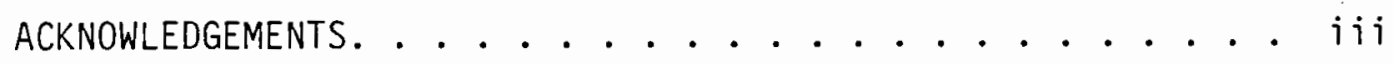

LIST OF TABLES. .............................. vi

LIST OF FIGURES .............................

CHAPTER

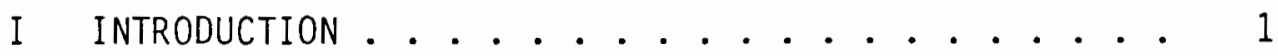

I I Literature REVIEW . . . . . . . . . . . . . . 14

Physical Attractiveness .......... 14

Attitude .............. 19

Application in Social Gerontology. . . . . . 22

II METHODS . . . . . . . . . . . . . . 25

Section I: Photographic Subjects...... 27

Section II: Selection Judges ....... 30

Section III: Questionnaire Judges ...... 35

Section IV: Subjects.......... 110

IV RESULTS. ................... 117

$\checkmark$ SUMMARY AND LIMITATIONS. . . . . . . . . . 141

VI IMPLICATIONS AND APPLICATIONS. . . . . . . 150 SELECTED BIBLIOGRAPHY . . . . . . . . . . . 156 APPENDICES

A Recruitment Notices............ 161

B Consent Forms............. 166

C Thank You Letters. . . . . . . . . . 173

D Questionnaires ............ 177

E Questionnaire Development. ......... 217

F Computer Code Sheets............ 219 


\section{APPENDICES}

G Selection Judges . . . . . . . . . . 238

H Stimulus Photographs . . . . . . . . . . 276

I Subjects . . . . . . . . . . . . . 280 


\section{LIST OF TABLES}

Page

TABLE

I Test-Retest Descriptive Statistics on Physical

Attributes Items (OFIQ)..........

II Test-Retest Descriptive Statistics on Instrumental Qualities Items (OFIQ).........

II Test-Retest Descriptive Statistics on Interpersonal Qualities Items (OFIQ)..........

IV Test-Retest Descriptive Statistics on Affect

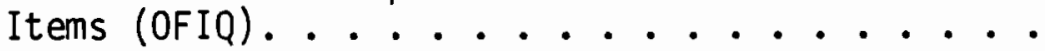

$V$ Test-Retest Descriptive Statistics on Behavior Pre-disposition Items (OFIQ). . . . . . .

VI Rotated Factors Matrix on Physical Attributes Items (OFIQ)............

VII Rotated Factor Matrix on Instrumental Qualities Items (OFIQ).............

VIII Rotated Factor Matrix on Interpersonal Qualities Items (OFIQ)............

IX Rotated Factors Matrix on Affect Items (OFIQ) . .

$X$ Rotated Factors Matrix on Behavior Predisposition Items (OFIQ)............

$X I$ Test-Retest Pearson $r$ Correlation Coefficients on Physical Attributes Items (OFIQ)........

XII Test-Retest Pearson $r$ Correlation Coefficients on Instrumental Qualities Items (OFIQ) ......

XIII Test-Retest Pearson $r$ Correlation Coefficients on Interpersonal Qualities Items (OFIQ)...... 
viii

Page

XIV Test-Retest Pearson $r$ Correlation Coefficients on Affect Items (OFIQ).............

XV Test-Retest Pearson $r$ Correlation Coefficients on Behavior Predisposition Items (OFIQ). . . . .

XVI Test-Retest Discrimination Indices on Physical Attributes Items (OFIQ)............

XVII Test-Retest Discrimination Indices on Instrumental Qualities Items (OFIQ)........

XVIII Test-Retest Discrimination Indices on Interpersonal Items (OFIQ)...........

XIX Test-Retest Discrimination Indices on Affect Items (OF IQ)................

XX Test-Retest Discrimination Indices on Behavior Predisposition Items (OF IQ)..........

XXI Descriptive Statistics on Modified First Impression Questionnaire (MFIQ) Items .........

XXII Discrimination Indices on the Modified InterPersonal First Impression Questionnaire (MFIQ) Items

XXIII Rotated Factors Matrix on Modified First Impression Questionnaire (MFIQ) Items.

XXIV Young Male Subjects Rating Young, Middle-Aged, 01d Female Stimulus Photographs . . . . . . . . .

XXV Young Female Subjects Rating Young, Middle-Aged, 01d Female Stimulus Photographs ... . . . . .

XXVI Young Male Subjects Rating Young, Middle-Aged, Old Female Stimulus Photographs .........

XXVII Young Female Subjects Rating Young, Middle-Aged, 01d Female Stimulus Photographs..........

XXVIII Young Male Subjects Rating Young, Middle-Aged, Old Female Stimulus Photographs 
Page

XXIX Young Female Subjects Rating Young, Middle-Aged, OId

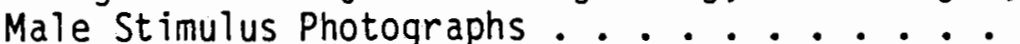

XXX Young Male Subjects Rating Young, Middle-Aged, Old Male Stimulus Photographs. . . . . . . . . .

XXXI Young Female Subjects Rating Young, Middle-Aged, Old Male Stimulus Photographs...........

XXXII Pearson $r$ Correlation Coefficients on the Affect

Component Associated with the Four Other Components.

XXXIII Descriptive Statistics of Young, Middle-Aged, 01d

Selection Judges Ranking/Rating Young Stimulus

Photographs' Physical Attractiveness .......

XXXIV Descriptive Statistics of Young, Middle-Aged, 01d

Selection Judges Ranking/Rating Middle-Aged Stimulus Photographs' Physical Attractiveness ........

$X X X V$ Descriptive Statistics of Young, Middle-Aged, 01d

Selection Judges Ranking/Rating 01d Stimulus

Photographs' Physical Attractiveness . . . . . .

XXXVI Descriptive Statistics of Young Middie-Aged, 01d Male

Selection Judges Ranking/Rating Young Stimulus

Photographs' Physical Attractiveness .......

XXXVII Descriptive Statistics of Young, Middle-Aged, 01d Male Selection Judges Ranking/Rating Middle-Aged Stimulus Photographs' Physical Attractiveness ........

XXXVIII Descriptive Statistics of Young, Middle-Aged, Old Male Selection Judges Ranking/Rating 0ld Stimulus Photographs' Physical Attractiveness. . . . . . . .

XXXIX Descriptive Statistics of Young, Middle-Aged, 0ld Female Selection Judges Ranking/Rating Young Stimulus Photographs' Physical Attractiveness . . . . . .

$X L$ Descriptive Statistics of Young, Middle-Aged, 0ld Female Selection Judges Ranking/Rating Middle-Aged Stimulus Photographs' Physical Attractiveness. . .

XLI Descriptive Statistics of Young, Middle-Aged, 01d Female Selection Judges Ranking/Rating 01d Stimulus Photographs' Physical Attractiveness . . . . . . . 
XLII Group Mean, Standard Deviation and Range of Young, Middle-Aged, 01d, Selection Judges Rating Male and Female Young, Middle-Aged and old Stimulus

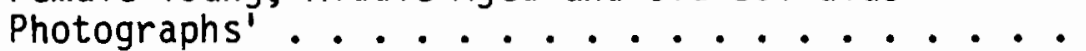

XLIII Group Mean, Standard Deviation and Range of Young, Middle-Aged, 01d Male Selection Judges Rating Male and Female Young, Middle-Aged and old Stimulus Photographs' ............ 274

XLIV Group Mean, Standard Deviation of Young, Middle-Aged, 0ld, Female Selection Judges Rating Young, Middle-Aged and 01d Stimulus Photographs' . . . . 275 


\section{LIST OF FIGURES}

Page

FIGURE

1. Levels of Paired Relatedness Theory ..... 5

2. A Schematic Conception of Attitudes...... 21

3. Original Number of Photographs Representing Stimuli Gender and Age Group. . . . . . . . 30

4. Distributions of Means before Paring. . . . . 33

5. Distributions of Means after Paring ...... 33

6. Matrix Representing Stimulus Photographs Physical Attractiveness Intensity Levels .......

7. Items on the Original First Impression Questionnaire

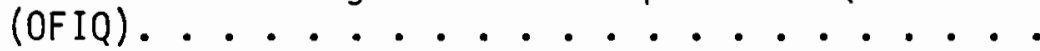

8. Items on the Modified First Impression Questionnaire

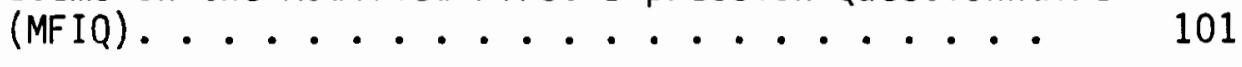

9. Matrix Used in Creating Six Photo Orders. . . . 112

10. Statistical Analysis Used on the Honeywell $66 / 40$ Computer to Validate the OFIQ and MFIQ. . . . .

11. Content Analysis of Selection Judges Tape-Recorded Responses Based upon 2 Questions Asked After Ranking the Stimulus Photographs..........

12. Photo Order 1 Physical Attractiveness Intensity Levels, Age Group, and Sex.........

13. Photo Order 2 Physical Attractiveness Intensity Levels, Age Group, and Sex.........

14. Photo Order 3 Physical Attractiveness Intensity Levels, Age Group, and Sex.........

15. Photo Order 4 Physical Attractiveness Intensity Levels, Age Group, and Sex......... 
16. Photo Order 5 Physical Attractiveness Intensity Levels, Age Group, and Sex..........

17. Photo Order 6 Physical Attractiveness Intensity Levels, Age Group and Sex........... 


\section{CHAPTER I}

\section{INTRODUCTION}

Across gender and across time, physical attractiveness has been a factor in nonverbal communication between human beings. From the nursery to the nursing home, individuals sustain a lively and unwavering interest in both their own looks and the physical attractiveness of others. But what is meant by the words "physically attractive"? In studies conducted to date, researchers have failed to settle upon a standard definition. Hard to measure and even harder to deny, first impressions of physical attractiveness play a very influential role whenever strangers judge strangers.

Physical appearance, if judged by the never-ending barrage from the advertising industry, is the paramount factor in nonverbal interpersonal communication. Even looked at from a calmer, more objective perspective, it is difficult or impossible to make a case against the importance of physical appearance in the shaping of expectations that we place upon unknown others and they upon us. Before any words are spoken, an individual's looks are his or her first form of nonverbal communication with the rest of the world, and the impressions conveyed in that first glance are powerful and lasting. 
All human communication which lies outside the realm of the spoken or written word is referred to as nonverbal communication (Knapp, 1980). A tremendous amount of information other than what is given to us in written or verbal symbols is transmitted through this nonverbal behavior. Given a contradiction between verbal and nonverbal communication, people rely upon nonverbal cues 65 percent of the time (Tabor, 1970; and Birdwhiste11, 1970).

Numerous researchers have broken nonverbal communication into different typologies (Knapp, 1980; Ekman and Friesen, 1969, 1972; Ekman, 1976; Knapp, Wiemann and Daly, 1978; Siegman and Feldstein, 1978; Weiner, Devoe, Rubinow and Geller, 1972; and Burgoon and Saine, 1978). How the categories are defined varies from author to author. However, the most accepted typology is Knapp's set of seven categories of nonverbal communication: (1) body motion; (2) phsyical characteristics; (3) touching; (4) paralanguage; (5) proxemics; (6) artifacts; and (7) environment.

This study deals with his "physical characteristics" category. Knapp holds that physical appearance on first meeting with a stranger determines whether or not that the stranger will want to know you.

Physical attractiveness plays an important role in persuading and/or manipulating others in relationships from nursery school on through courtship and in the job market, but prior research findings dealing with physical attractiveness have been vague and contradictory. Although Chapter II will review the literature on 
physical attractiveness and describe some of the vagueness in this area, the investigator will touch here upon some of the more common definitions of "attraction."

Marlowe and Gregen (1969) define attraction as "...those instances in which a person responds in an emotionally positive way to another", but they admit, in the next sentence, "such a definition can be considered orienting only in a grossly general and imprecise way...".

Hendrick and Hendrick (1983) define attraction as:

"...an orientation toward or away from a person that may be described as having valence (positive, neutral or negative). The orientation consists of a cognitive structure of beliefs and knowledge about the person, the affect felt and expressed toward him or her, and behavior tendencies to approach or avoid this person" ( $p, 10)$.

These later authors' reference to a cognitive structure, affect and behavior tendencies describes what the layman would call an "attitude".

Attraction is considered by Hendrick and Hendrick to be a special type of attitude towards another person or an object, and, in fact, most research in the area of attraction has dealt with feelings a person has experienced about a stranger in a photograph. Most findings have been derived from superficial relationships created in a laboratory. This type of research creates and studies a "person object relationship". "Person - object relationship" refers to the initial awareness that another person exists besides yourself (Hendrick \& Hendrick, 1983). 
The evaluating person is asked to make a positive or negative statement about a person (referred to here as the object) in a photograph, based merely on physical appearance. We all do this on a conscious or subconscious level with people we meet or see in newspapers, magazines and television. The evaluating person has little or no information about Person B except their physical characteristics. When a new face is encountered, we generalize our past experience in relationships, remembering people who communicated nonverbal impressions similar to those of the new person we are looking at. We project old messages and accumulated stereotypes onto the people we encounter daily. When a person meets a stranger, he/she will evaluate first their physical appearance and then their verbal skills. The person will determine from this scanty amount of information if he/she would like to continue a relationship with the "object" person. This study deals with the person - object relationship (see Figure 1), the first level in Levinger and Snoek's theory (1972) of "Levels of Paired Relatedness".

The second level in the three-part theory is referred to as "surface contact", with limited interaction. This stage can be defined as person - object "interaction". Notice; it is person object "interaction"--not a person - object "relationship"--and the emphasis is on what actually takes place in surface contact situations. For example, a stranger asks to borrow a pencil and the pencil-owner responds by passing the pencil.

The third stage, mutuality, includes open communication, self disclosure, commitment and symmetry. This is the level at which 
0. Zero contact
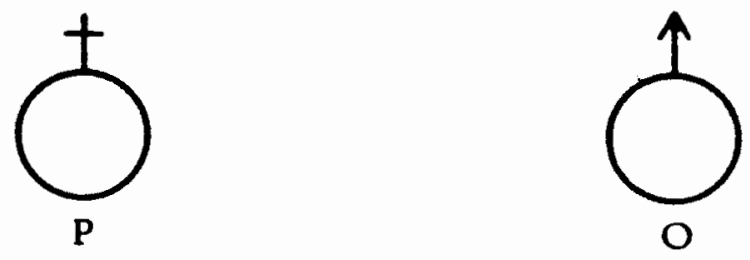

1. Awareness
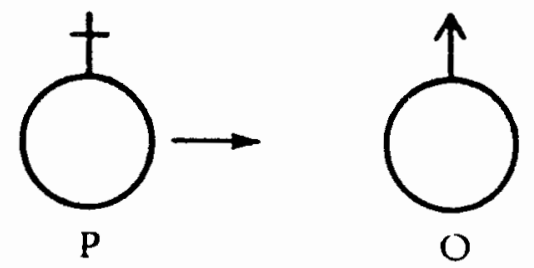

2. Surface contact

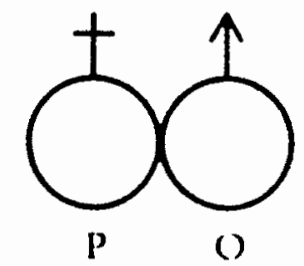

3. Mutuality

a. Minor intersection

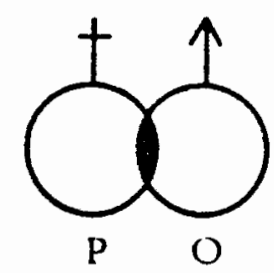

b. Moderate intersection

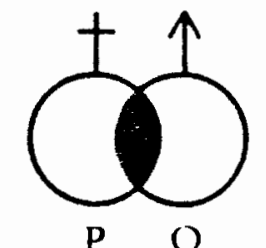

c. Major intersection

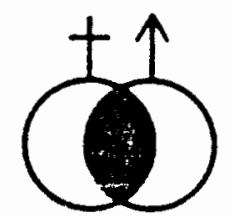

Figure 1. Leveis of Paired Relatedness Theory. Based on Attraction in Relationship: A New Look at Interpersonal Attraction (Levinger and Snoek, 1972). Copyright. Reprinted by permission. 
both people have shared knowledge of each other. The level of involvement in the third stage is indicated by the shaded area of the two circles in Figure 1.

This study attempts to cast light on the nonverbal components of communication (Knapp) inherent in the person - object relationship. It does so by scrutinizing those attitudes that individuals call upon daily when forming first impressions of strangers. Its major contribution may be its two-pronged perspective; a view that looks at not just those pretty young females, but also at males and at the generational width and breadth of "America's over-25 population".

\section{STATEMENT OF HYPOTHESES}

The hypotheses of this study are the following:

(1) The Young Female stimulus photograph will be rated more positively in physical attractiveness than either the Middle-Aged or 01 der stimulus photographs by subjects in the 19 to 24 year old age group, (Young).

(2) Young subjects will evaluate the Middle-Aged Male in the stimulus photographs as being more physically attractive than either the Young or 01d Male stimulus photographs.

(3) The more the person in the stimulus photograph is liked (indicated by a high score in Affect), the higher young subjects of either gender will rate the stimuius photograph in the other four components. 
OPERATIONAL DEFINITIONS

The following operational definitions were used throughout this investigation. The definitions appear in the order in which they are first introduced in the text of the study.

\section{Physical Attractiveness}

Hendrick and Hendrick's definition of physical attractiveness was based on an "attitude" that consisted of a cognitive, affective, and behavioral structure. Most investigators have defined an "attitude" as having these three components. But physical attractiveness in this investigation refers to an attitude or feeling toward another person, whether positive, neutral, or negative. The investigator defined physical attractiveness multidimensionally with five analyzable components: (1) physical attributes; (2) instrumental qualities; (3) interpersonal qualities; (4) affect; and (5) behavior predisposition. The following definitions describe those five components.

\section{Physical Attributes}

The Physical Attributes Items on both questionnaires referred to external physical characteristics such as eyes, mouth, face shape, hair, and so forth. They were derived from the judges' selection based on tape-recorded opinions of what physical characteristics they found very physically attractive or very physically unattractive. 
These comments $c$ an be found in Appendix $G$ and arranged in Figure 11, which shows the full spectrum of responses across age and gender.

\section{Instrumental Qualities}

The Instrumental Qualities component referred to the presumed non-interactive qualities of the photo subjects that is the nonsocial aspects of their personal character. It would include internal qualities such as warmth, enthusiasm, sophistication, or kindness. Instrumental qualities are a cognitive component.

\section{Interpersonal Qualities}

The Interpersonal Qualities component on the questionnaire reflected the anticipated sociability of the participant towards the stimulus photographs in an imagined working situation. Qualities such as cooperation, pleasantness, and consideration are the social aspects of personal character: that is, the ability to get along with others. They may also be considered cognitive in character.

\section{Affect}

The Affect component sought to measure how much stimuius photographs were liked or disliked. This component defined the amount of "liking" by means of a scale ranging from polarities of "Strongly Agree" (7), to "Strongly Disagree" (1).

Behavior Predisposition

The Behavioral Component referred to an individual tendency to approach or avoid a particular situation or person. It can be 
defined as the intentions contained within a person's overt behavior, either verbalized or shown nonverbally, toward another person. These items represented a variety of self-perceptions about communicative tendencies and guesses people may make about their potential relationships. In this study, Behavior Predisposition items measured the judges' and subject's projected behaviors toward the stimulus photographs.

Participants in the Study and the Populations

Photo judges. The investigator created an initial group of 48 photographs from which this study's final set of 18 stimulus photographs would be selected. The task of the photo judges was to achieve standardization of photographic subjects' facial expression. Ten college-educated Caucasian participants were recruited to select a photograph out of each set that maintained consistency with facial expressions in all 48 photographs.

Selection judges. A description of the selection judges can be found in Chapter III. The tasks of the judges were to pare down the number of photographs to be used in the study; to help eliminate any investigator bias in stimulus photograph selection; and to develop a control for physical attractiveness by providing the data which would enable the investigator to create three intensity levels of attraction.

Stimulus photographs (subjects). Stimulus photographs consisted of $18(5 \times 7$ inches) colored photographs showing only the 
full-face and necks of Caucasian, college-educated individuals who were unknown to the rest of the participants. The age classifications of the stimulus photographs were Young (19-24), Middle-Aged (42-47) and 01d (65-70 years). The people in the photographs represented three intensity levels of physical attractiveness: high, medium, and low. Each age group and both genders are represented in the photos at all three intensities of attractiveness.

A preliminary part of this study reduced the original 48 photographs to the final 18 used to address the hypotheses.

Questionnaire judges. The questionnaire judges were instrumental in modifying the original multi-dimensional questionnaire. Their feedback was essential because the data they provided was used by the investigator to pare down the 105 items on the Original Questionnaire, reducing them to the final 30 items selected for the Modified version. This set of judges was not part of the population sampled as subjects, previous judges, or stimulus photographs. It was a separate group of people who were individually tested and retested to validate all items on the Original Questionnaire.

Subjects. The actual study subjects consisted of 72 "young" participants between the ages of 19 and 24 years. There were 36 males and 36 females within this group. The subjects' task was to address the initial research questions and hypotheses of this investigation using the Modified First Impression Questionnaire (referred to as MFIQ). 
Gerontology

Gerontology refers to the study of the aging process. Gerontologists examine the biological, psychological, sociological and economic concerns and needs of the elderly. Gerontology is both an applied and an academic discipline. Most gerontologists agree that aging begins at birth and continues until death, in a lifelong process, but, in general, gerontological researchers deal with people who are 65 and older.

This study touches on a discipline called "Social Gerontology", which deals with the social and cultural factors that effect the aging process. The term "Social Gerontology", although coined in 1950, has only recently become a subspecialty of gerontology. When this study looks at its subjects' evaluations of the nonverbal messages conveyed by the faces of the elderly, it crosses into the realm of social gerontology. The social implications of physical attractiveness and/or its decline among the "older" population are many.

\section{Age ism}

Ageism refers to the process of systematically stereotyping and discriminating against people because they are "old". Negative perceptions of the aged have arisen from myths and stereotypes similar to those involved in racism or sexism, and may certainly involve the changed physical characteristics of older generations. 


\section{Cohort Effect}

The social changes an individual experiences throughout his life are referred to as the "cohort effect". A cohort may aiso refer to a member of a group of people who are not the same chronological age but who do share experiential history (Ryder, 1965, 1968 and Cain, 1967, 1968). A birth cohort consists of people born at the same time in history who have shared many of the same life experiences (Hendricks and Hendricks, 1977; Cain, 1979).

\section{BRIEF OVERVIEW OF THE STUDY}

Several phases were involved in the task of proving or disproving the hypotheses of the study. The first general phase began with the selection of photographic subjects, progressed into the creation of a set of photographs which were to become the stimuli, and proceeded to the selection of the photo judges, whose evaluations would determine the final select group of 18 photographs and their physical attractiveness intensity levels.

Next, an initial questionnaire was designed and constructed, then tested and retested to ascertain its inter-item reliability. The first questionnaire differed from all its predecessors in physical attractiveness research because of its unique multidimensional approach. It was followed by a modified questionnaire that resulted from statistical analys is of the original version.

The final phase in testing the three hypotheses involved 72 subjects who, by completing the modified questionnaire, provided 
the necessary data for an in-depth statistical analysis of physical attractiveness across age-group and gender as perceived by subjects in the 19-24 year old age group.

The statistics thus obtained are intended to cast light upon the subject of physical attractiveness in a manner less subjective and more scientific than prior approaches. 


\section{LITERATURE REVIEW}

In reviewing the enormous amount of literature on physical attractiveness, it is soon apparent that younger females are more physically attractive, desirable and likeable than other groups in the general population (Miller, 1970). However, little supportive attention has been given to physical attractiveness across age groups, an omission that tends to make the above statement rather inconclusive. In addition, the research that has dealt with physical attractiveness in rating someone's physical appearance has been very "subjective" rather than "objective". To say that a person is very physically attractive may mean something to the individual who says it but does not say anything about what makes that person appear that way.

This research study examines the elusive quality of physical attraction using five components that combine to define physical attractiveness more exactly. The researcher's goal has been to learn--empirically and objectively--what makes a young, middle-aged or older person physically "attractive" or "unattractive" to a young person in the 19 to 24 year old age group, in part by the use of carefully selected photographs. 
The importance of physical attractiveness as a factor in person perception has been demonstrated as early as nursery school. Lerner and Geller (1969) for example, found that preschoolers were already expressing an aversion to the body types of their chubby classmates. Dion and Berscheid (1972) found that unattractive nursery school boys were liked less than attractive ones and were characterized as being more aggressive and antisocial. In addition, attractive children were seen as being more independent than unattractive children.

The strong relationship between physical attractiveness and popularity continues through grammar and high school into young adulthood. A common finding in studies of dating among college students is that, in general, attractive dating partners are preferred over unattractive ones (Berscheid, Dion, Walster and Walster, 1971). Also, it seems to be the case that physical attractiveness is more important to men than to women. Contrary to this finding, Murstein (1972) found physical attractiveness operates with exchange market rules. In other words, individuals with equal market values of physical attractiveness are more likely to associate in an intimate relationship. Huston (1973) found that a man's rating of his own attractiveness determined his perceived chances of acceptance by a potential date. Those who rated themselves as being highly attractive perceived their chances to be better than did those who saw themselves as being unattractive. (Ironically, Murstein's (1972) research with young adults and Cavior's (1970) research with grammar and high school students has 
shown that the correlation between self-ratings of physical attractiveness and the opinions of outside judges is quite low. It would be interesting to test whether attractiveness self-rating accuracy improves in old age, or whether the correlation between self-ratings for external judges remain low. Perhaps the wisdom which is said to come with advanced age overcomes the vanity or humility of youth.)

Apparently, judges share a set of learned social norms for physical attractiveness, as demonstrated in young adults (Murstein, 1972), grammar students and high school students (Cavior and Dokeck, 1971). A prominent aspect of these social norms is that female faces and adolescent faces are rated the highest in attractiveness by judges ranging from seven years of age to adul thood (Cross and Cross, 1971). According to Udry (1965), most people have a common standard when judging what features are considered physically attractive within their own culture.

Along with social norms for what is attractive, people develop physical attractiveness stereotypes which, in part, determine their behavior toward others. Dion, Berscheid, and Walster (1972) showed that attractive people were more likely to be expected to possess such socially desirable characteristics as sexual responsiveness, modesty, and kindness. These stereotypes are developing as early as nursery school (Dion and Bersheid, 1972) and persist into adulthood. Due perhaps to the stereotyping behaviors of others, physical attractiveness can have important consequences on the developing self-concept. Secord and Jourard (1953) and Rosen and Ross (1968), 
for example, found a positive relationship between satisfaction with physical characteristics and satisfaction with non-physical characteristics, and Kaat and Davis (1970) found that attractive women considered themselves better dates, more feminine, more self-confident, and more likeable than did women of medium or low attractiveness. Kagan (1964) cites evidence of the same phenomenon in adolescents.

Berscheid, Walster and Campbel1 (1972) found that the more attractive a middle-aged female was judged to be from college photographs of 20 years earlier, the less happy and well-adjusted she was in her current middle years of life. Contrary to this prior finding, an "adaptation level" theory explanation (Helson, 1964; Thibaut and Kelly, 1965) found that women who were "attractive" in college have greater happiness than women who were "unattractive." This explanation fails to take into account current physical attractiveness.

If, for example, physical attractiveness wanes as one approaches old age, the self-esteem of previously attractive people may deteriorate more than that of previously unattractive people, since the previously attractive had more to lose to begin with. This hypothesis could have been examined by Berscheid, Walster, and Campbel1 (1972) had they obtained data on current attractiveness as well as on college-age attractiveness.

A complication arises in the above hypothesis, however, as indicated by Miller's (1970) finding that physical attractiveness loses its importance as a factor in impression formation when the 
level of attractiveness is low. It may be the case that as physical attractiveness decreases with old age, its importance decreases also. Miller's (1970) procedure could be used with older stimulus persons to determine whether this is so.

Changes in the other potential measurements of attractiveness may be evident in old age even if physical attractiveness does not decrease in importance. Attractiveness norms for older stimulus persons will probably differ from those for young adults; differences might also be found between older and younger judges of attractiveness. Both of these effects could be found using the procedure of Cross and Cross (1971) with older stimulus persons and other judges included in the design.

Changes could also be expected in physical attractiveness stereotypes when older stimulus persons are involved. For example, attractiveness might no longer indicate a "sexually responsive" person, while it might now indicate a "healthy" person (or some other stereotypical, socially desirable characteristics of older persons). It would be interesting to determine whether older judges would share the same physical attractiveness stereotypes about themselves as younger judges would; this could be done using the procedure of Dion, Berscheid, and Walster (1972).

The question still remains; "What is physical attractiveness?" Most investigators have defined physical attractiveness as a "positive attitude," (Berscheid and Walster, 1977; Hendrick and Hendrick, 1983; and Marlowe and Gregen, 1969) leading logically to the next question raised by the investigator: "What is a Positive 
Attitude?" The following section on attitudes discusses and defines what an attitude is. The questionnaire used in this study was designed by the investigator based upon the definition of an attitude.

\section{ATTITUDE}

According to Berscheid and Walster (1974), an attitude reflects a person's readiness to respond toward an object or person in a positive or negative manner. Katz and Scotland (1959) define an attitude as: "an individual's tendency or predisposition to evaluate an object or the symbol of that object in a certain way."

Zimbardo, Ebbesen, and Maslach (1977) indicate that an attitude is an internal state expressed overtiy in verbal or nonverbal behavior. A verbalized attitude about a person or object is an opinion.

Most investigators have defined an attitude as having three components: (1) cognitive, (2) affective and (3) behavioral (Berscheid and Walster, 1974; Zimbardo, Ebbesen and Maslach, 1977 and Hendrick and Hendrick, 1983). The cognitive component of an attitude consists of factual knowledge of the person or object being evaluated. It is the person's supposed personal beliefs, thoughts or ideas which are weighed by the evaluator on an associative basis. For example, a person evaluating another person in a photograph may say, "This person is kind", based upon prior experience and contact with someone he/she may have encountered in earlier life who looked similar to the person in the photograph. 
The cognitive component in this study was broken down into two parts; (1) Instrumental Qualities and (2) Interpersonal Qualities (refer to Figure 2). The other three components of the multidimensional approach were: Physical Attributes--that is, the external physical characteristics of a person; the Affective component--that is, the amount of "liking someone" (ranging from polarities of strongly agree, 7 to strongly disagree, 1); and the Behavioral component--that is, the intention of the person's overt behavior, either verbalized or as an action towards another person.

The Behavioral component was measured in the Original first Impression Questionnaire (referred to hereafter as $O F I Q$ ), in question such as Item 68: "If I were with this person in a social conversation, I would direct the course of it." In other words, the Behavioral Component of an attitude refers to the individual's behavioral tendency to approach or avoid a particular situation. Figure 2, a schematic diagram illustrating the three components of an attitude (Rosenberg and Hovland, 1960; Zimbardo, Ebbesen, and Maslach, 1977) was used in defining physical attractiveness in this investigation. The preceeding material can be illustrated in this diagramatic figure on attitudes.

Most researchers have assumed that physical attractiveness is an unidimensional variable (Huston, 1973). A unidimensional approach denies other factors that may influence a person's perception in evaluating physical attractiveness. The unidimensional view does not recognize the fact that one's attitude toward an individual may be extremely complex, or that we may feel different 


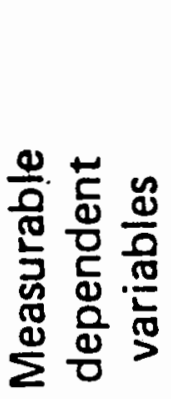

号 $\frac{0}{\frac{\Phi}{2}}$

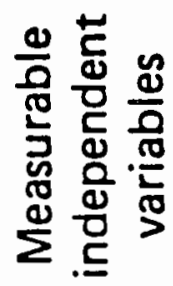

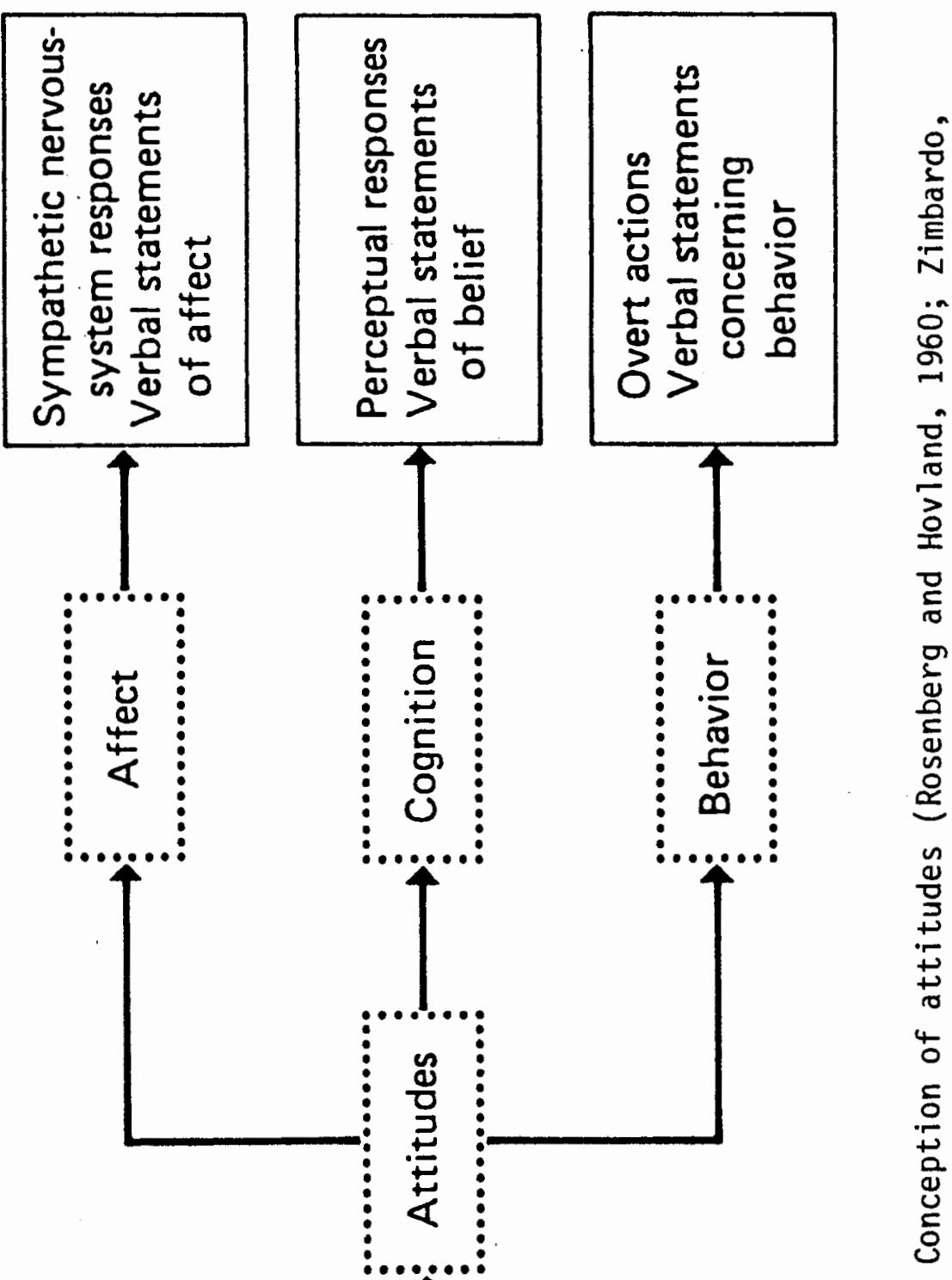

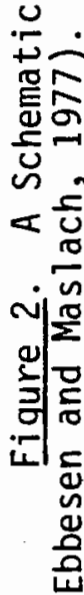


kinds of attraction for others (Berscheid and Walster, 1974). The investigator of this study believes and has shown that there are more components to physical attractiveness than external qualities which are merely "skin deep".

APPLICATIONS IN SOCIAL GERONTOLOGY

Most of the prior research conducted on physical attractiveness has compared relationships between child and child, adolescent and adolescent, or young adult and young adult. There appears to be little research on physical attractiveness across generational lines or strictly among the "old" (Adams and Huston, 1975; Johnson, 1984). Few studies have dealt with physical attractiveness in the middle-aged years (Adams and Huston, 1975; Johnson, 1984). It may be the case that as physical attractiveness decreases with old age, its importance decreases also. It would be interesting to determine whether older subjects would share the same physical attractiveness stereotypes about themselves as young people. Is physically attractiveness effected by "cohort differences" or by a person's "perceived age"? Given the evidence to date, it seems reasonable to suggest that all individuals may suffer dissonance when external physical changes occurring in their life cycles collide with their long-standing inner perception of their physical appearance. As physical features change, many individuals enter a "crisis period". It may be suggested that it is during this crisis period when he or she may be most susceptible to the negative socio-psychological 
after-effects of having internalized society's norms of physical attractiveness.

Due to a lack of information, individuals attribute characteristics to others merely on first impressions of a person's physical appearance in order to minimize ambiguity and to gain social control of the situation.

Sontag (1980) has suggested that modern urbanized society has emphasized youth as the ultimate age group of physical attractiveness. With our ever-increasing age groups of middle-aged and older people whose life-spans have been extended by modernization of medication, sanitation, and nutrition (Hendricks and Hendricks, 1977), the older generation will soon out-number its younger "cohort".

In the past two generations, the situation of older people in the United States has changed drastically. There are more older people living now than at any other time in our history and their numbers are growing faster than any other age group. In 1900 only four percent of the population was over the age of 65 . By 1950 the figure had risen to 8.1 percent (Hendricks and Hendricks, 1977). Today the current older population is between 9.1 and 9.4 percent (Bureau of Census, 1984), and in the year 2000, the Bureau of Census (1984) has projected it at 24.9 percent. Will the new masses of older Americans try to maintain a "youthful image" by washing away that gray in their hair or will there be a reverse effect of younger people attempting to look old by putting that gray back in their hair? Advertising plays an influential role in determining cultural 
norms for physical attractiveness among the young. Research by Brown (1982) shows that middle-age has been overlooked for a lack of demographic data and a means of determining an accurate definition. Do older and middle-aged people perceive physical attractiveness differently within their own age groups?

This study attempts to broaden the literature on Physical Attractiveness by adding the well-weathered planks of middle-aged and older aged men and women. But much work remains to be done. It is quite possible that as the older majority surfaces, cultural norms of physical attractiveness may change drastically. 


\title{
CHAPTER II I
}

\author{
METHODS
}

\section{Introduction}

The procedures presented in this chapter address the methods used in answering the research questions. Due to the quantity of material and the complexity of this study, the methods material has been separated into four phases:

Phase I, involving creating the photos of people in different age groups which would be used as stimulus materials.

Phase II, dealing with selection judges to rate and rank stimulus photographs according to their physical attractiveness.

Phase III, describing how the questionnaire was developed based on the responses in Phase II, and also describing a different group of judges who reduced the number of $i$ tems on the questionnaire.

Phase IV, describing how subjects rated the stimulus materials by completing the questionnaire to test the hypotheses of the study.

Al though there were different phases in the methodology of the study, there were some procedures common to all of them. These are described in the following pages. 
Selection Criteria for All Phases

The ethnic, socio-economic and educational backgrounds of all participants in the study were taken into consideration in order to control those variables. Comparability of background was established through use of a socio-economic status questionnaire (hereafter referred to as SES) which was administered to all participants of each phase of the study. The SES was a modified version of Dillman's Mail and Telephone Surveys (1978). Eight items appeared on the SES, asking standard questions pertaining to sex, age, income level, level of educational status, student's status (current), occupation and address (see Appendix D).

The results of this survey allowed the investigator to select participants who were similar in regard to the items. An exception was in regard to sex, where equal numbers of men and women were selected for each phase.

\section{Recruitment}

Participants were recruited from Portland area colleges, senior centers and the Adult Learning Center at Portland State University. Presentations about the study were made to interested participants at those locations. In order to obtain names and phone numbers of people willing to participate in this study, sign-up sheets were provided. The investigator scheduled appointments on campus at Portland State University to interview all participants. All potential 
participants were given a standardized two paragraph description of the task, location and required time for participation: See Appendix A.

\section{Prel iminary Forms}

The investigator was present while a consent form was signed and was able to answer questions pertinent to participation.

A second item each person received was a "Note on Anonymity," assuring participants that all obtained information would be identified by a code (see Appendix B). Next, an SES questionnaire was given to each participant. After all the items on the questionnaire were completed, the participant was ready to begin the assigned task. Standardized verbal descriptions of relevant phases of the study were given to all participants.

Within a week of the judges' and subjects' participation, a "Thank You" letter was mailed (see Appendix $C$ ). Participants were al so promised an abstract of the report upon completion of the study.

The following four sections address each phase of the study's methodology, respectively.

\section{SECTION I}

Section I describes how the photographs were created. These photographs, referred to as stimulus materials, were used throughout the study. This section describes the photographic subjects, procedures and the standardizing of the facial expression in the photographs. 
Creating Stimulus Photographs: Photographic Procedures

Age group of photographic subjects. Photographic subjects

consisted of three age groups: Young (19-24), Middle-Aged (42-47) and $01 \mathrm{~d}(65-70)$. There were sixteen participants in each age group (eight males and eight females), for a total of 48 photographic subjects. These age groups, divided by intervals of 16 years, were arbitrarily selected by the investigator in the interest of creating groups in which physical characteristics of photographic subjects were clearly distinct and identifiable.

Photo session. An effort was made to have a similar room, lighting, and background at each college campus and the senior center. The photographs were taken with a Mamika C-330 camera with flash equipment.

The posture of the participants was held constant. All the stimulus photographs were of the head and shoulder area with facial emotional expressiveness ranging from passive to smiling. A professional photographer took three portraits of each subject (referred to as a set).

Standardizing Expression of Photos

The investigator needed to control the facial expressiveness of the photographic subjects to eliminate extremes of smiling or frowning. The purpose of this control was to eliminate other variables besides basic physical appearance. Photo judges examined three photographs of each person and selected one picture. The 
selected photograph was to be comparable in facial expression to the stimulus photographs of the other subjects.

The procedures below were used to achieve standardization of expression.

Photo judges. Ten college-educated Caucasian participants (five males and five females) were recruited in the same manner as described for the recruitment of the photo subjects. The ages of the photo judges were not controlled. Preliminary forms (Consent Form, SES, A Note of Anonymity) had to be completed prior to beginning the task. Data sheets were provided for all judges to record their photographic choices.

Photo selection. The photo judge was to select a photograph out of each set that maintained consistency with the facial expressions in all 48 sets. The selected photograph was recorded by number on the back of the photograph and recorded on the data sheet. This process was repeated with each set until all 48 photographs had been selected. The final photo product consisted of 48 single-shot photographs.

\section{Final Photo Product}

Size and format. All stimulus photographs were $5^{\prime \prime} \times 7^{\prime \prime}$, colored photographs. They were placed in five-and-half by eightand-half, off-white mats, showing the full-face portraits. A threeinch, press-on letter was placed on the left-hand side to identify the photo. Figure 3 on the following pages summarizes data on the stimulus photographs. 
CHRONOLOGICAL

AGE

Young (19-24 years)

Middle-Aged (42-47 years)

$01 \mathrm{~d}(65-70$ years $)$
MALES

8

8

8
TOTAL NUMBER OF PHOTOGRAPHS

FEMALES

8

16

8

16

8

16

Number of Stimuti, $\mathrm{N}=48$

Figure 3. Original number of photographs representing stimulus photographs gender and age group.

\section{SECTION II}

Section II describes the final selection process of the 18 photographs to be used throughout the study. The pictures used in the first phase are the same pictures used in this phase. The selection process was aimed at selecting a representative sample of physical attractivenes across age groups. All three age groups were included in this phase to determine what was physically attractive to each group.

Selection Judges

The judges were 18 participants; three males and three females within each age group, for a total of six young, six middle-aged and six old judges.

Presentation of Stimulus Photographs to Selection Judges

One male and one female selection judge in each age category of young, middle-aged, and old composed what the investigator termed a "set." The first set of judges examined the 19-24 year old (Young) 
stimulus photographs; the second set of the same judges' age group examined the 42-47 (Middle-Aged) stimulus photographs and the third set evaluated the 65-70 year old (01d) stimulus photographs.

Selection Judges Evaluating Stimulus Photographs

The outcome of this process was that each of the 48 stimulus photographs was examined three times, once by one set of selection judges from each of the three age categories. In other words, every age group of stimulus photographs was examined by every age group of the selection judges.

Gender of stimulus materials. Photographic subjects were presented to the selection judges with females first, then males. The purpose of maintaining this order of presentation was to eliminate any uncontrolled order-effect presentation.

Procedures Selection Judges Used in Evaluating Stimulus Photographs

In order to evaluate the stimulus photographs' physical attractiveness, each judge was assigned the tasks of rating and ranking the pictures.

Rating. Physical attractiveness of photographic subjects was rated by each selection judge on a seven point scale ranging from "Very Physically Attractive (1)," to "Very Physically Unattractive (7)." (A copy of the scale can be found in Appendix D). This type of scale and the standard seven points were used throughout the study to assure internal consistency. 
Ranking. Each judge was asked to rank the 16 photographs using a hierarchy (see Appendix D). The investigator gave the following instructions for ranking the stimulus photographs:

Rank the same 16 photographs of who is most physically attractive to most physically unattractive spreading them out on the table. Again, females are first to be ranked and then males.

The investigator recorded the stimulus photographs numbers in their ranked order on an observer sheet while identifying the participating selection judge with a number (refer to Appendix D).

Selection judges ranked the stimulus photographs to make comparisons of all the pictures' physical attractiveness in that particular age group rather than evaluating the pictures on an individual basis, which occurred in the rating procedure. Verbal comments made by the selection judges were tape-recorded and transcribed to be used later as part of the questionnaire development (refer to Section III).

Paring. The range of physical attractiveness of pictures in each age group was pared down to create comparable end points for physical attractiveness across age groups as illustrated in Figure 4 and Figure 5 on the following page.

Another effect of paring the stimulus photographs was to eliminate extremes of high and low physical attractiveness while maintaining a range of intensities within each age group.

The paring was accomplished by eliminating the extreme rating and ranking means that fell outside the defined "window" within 

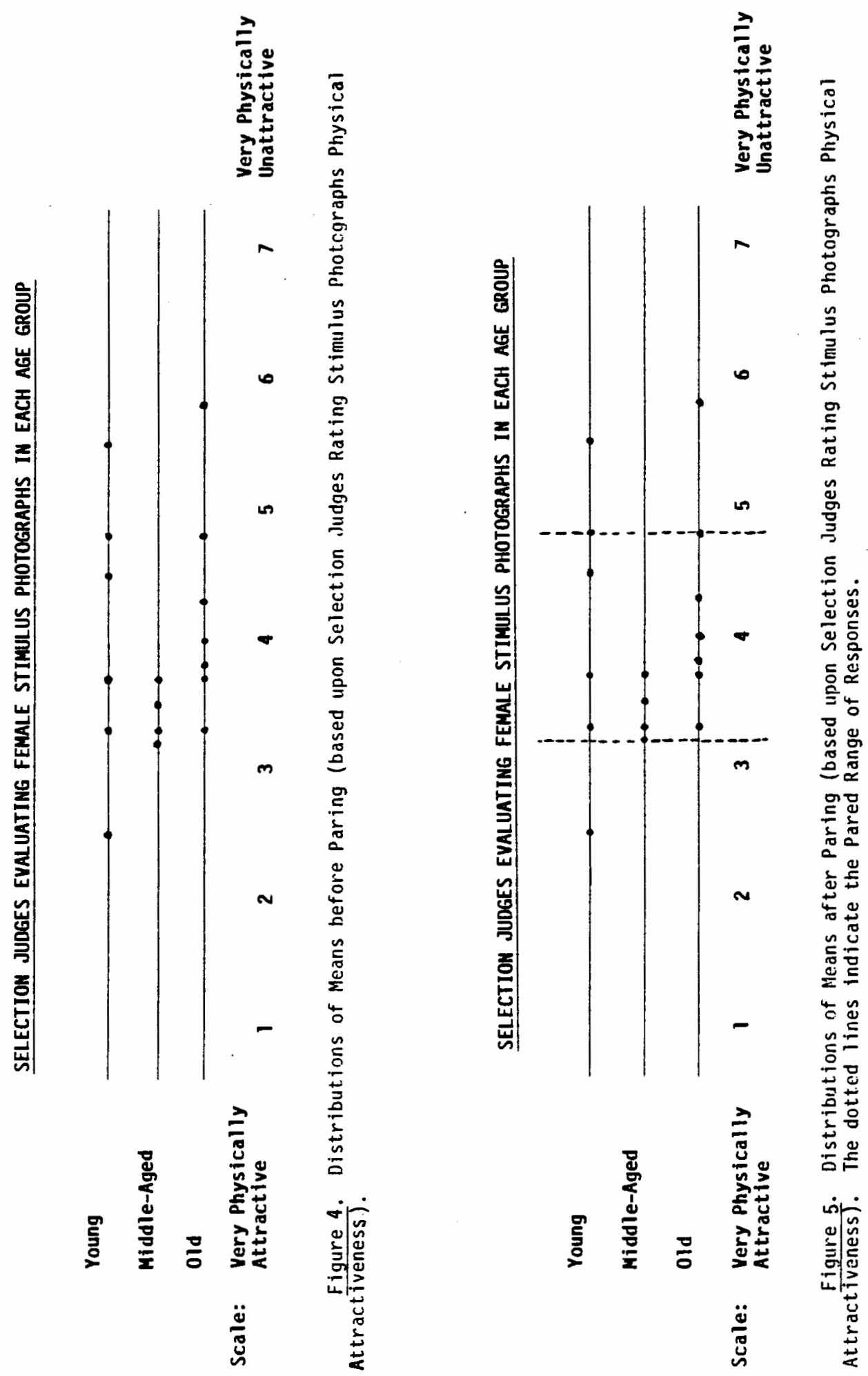
each age group continuum. In paring the photographs down to their final number, preference was given to a larger standardization which indicated larger variability in judgements of physical attractiveness.

The statistics used in reducing the number of photographs were hand-computed descriptive statistics consisting of the mean and standard deviation as shown in Table XXXIII through Table XLI (see Appendix G).

Table XLII through Table XLIV reflect the group means, standard deviations and ranges of the selection judges rating stimulus photographs. In summary, the overall group means, standard deviations and ranges of the selection judges rating stimulus photographs can be seen on Table XLII (Appendix G) with all three age groups broken down into male selection judges, female selection judges and total (a combination of male and female selection judges). The stimulus photographs had means of: Young Males 4.59, Females 3.91, and Total 4.29; Middle-Aged Males 3.63, Females 3.46 and Total 3.58; and 01 der Males 4.08, Females 4.36 and Total 4.26. Selection judges range for rating Male photographs were from 2 to 5 , Female 2 to 7 , and Total 2 to 7. The stimulus photographs standard deviation based upon the selection judges in all three age groups was for male selection judges 1.36 to 1.38; female selection judges, 1.24 to 1.77 , and Total, 1.25 to 1.56 .

The photographs were selected based upon the means that fell inside the "window" within each age continuum. So, all selected 
photographs would be comparable across age group in physical attractiveness. In summary, these findings indicated wide variability of opinion. The selection judges' evaluations showed an amount of deviation that would indicate that their selections of stimulus photographs were similar to those which might be expected of the general public.

\section{SECTION III}

Section III describes how the questionnaire was developed, tested, and retest. It first describes development and construction of the questionnaire (creation of a matrix for presenting the stimulus photographs). Next, it looks at selection of questionnaire judges, presentation of the stimulus photographs to questionnaire judges, testing and retesting of the questionnaire and interpretation of statistical analyses.

\section{Questionnaire Judges}

The questionnaire judges were 12 participants: there were six males and six females within the young and old age group.

Questionnaire Development and Construction

Questionnaire development and construction was based on the idea explained earlier that physical attractiveness is a positive attitude with multidimensional components. The questionnaire developed in this phase broke down "a positive attitude" into five components containing (1) physical attributes, (2) internal 
qualities (described later as instrumental qualities), (3) interpersonal qualities, (4) affect and (5) behavior predisposition. The physical attractiveness questionnaire was called "Original First Impression Questionnaire" (referred to hereafter as OFIQ). OFIQ contained a total of 105 items. A description of how the components were developed follows.

Development of the Five Components

Physical Attribute items were based upon the tape-recorded responses (Phase II) of the selection judges evaluating the person's physical attractiveness in the photograph. Items referring to "Instrumental Qualities", "Interpersonal Qualities", "Affect" and "Behavior Predisposition" are components that constitute an attitude, (Rosenberg and Hovland, 1960), as explained in Chapter II. This was how the five components on the questionnaire were created. How the items were developed under each component will be discussed below.

\section{Physical Attributes}

Physical Attributes on the questionnaire were item numbered and labeled at the end points, Very Physically Attractive (7), to Very Physically Unattractive (1).

The physical attributes items on the questionnaire were based upon tape-recorded responses of the selection judges given during Phase II. After the selection judges rated and ranked the photographs within each age and gender, they were tape-recorded and asked the following two questions: 
(1) What is it about that person that you find very physically attractive?

(2) What is it about that person that you find very physically unattractive?

Categories of the tape-recorded responses were transcribed and grouped according to general characteristics such as "Face Shape" for comments like an oval face and a nice face (see Appendix G). There were two repeated items phrased differently for internal reliability; Item (1) Physical Appearance and Item (22): This person is: Very Physically Attractive to Very Physically Unattractive. Item 22 was used throughout the study as a validation measure to check that the responses to the stimulus photographs' physical attractiveness intensity levels were consistently perceived by questionnaire judges and subjects.

\section{Cognition}

Instrumental qualities. Instrumental items focused on internal qualities of the person in the photograph such as warmth, enthusiasm and kindness. As shown in Appendix D, Questionnaires: OFIQ, the instrumental qualities on the questionnaire can be found in Section II, items $23,25,27,29,32,33$ and 35 (a total of 7 items). Instrumental items were written as, "This person appears

_." ranging from positive (7) to negative (1) describing a given trait or characteristic such as independence versus dependence and open-mindedness versus closed-mindedness. Instrumental components of physical attractiveness items were taken from a pre-existing semantic 
differential scale (Petersen, 1976) and modified in format only to fit the design of the questionnaire addressed in this study. Interpersonal qualities. Interpersonal components on the questionnaire referred to the feelings of sociability felt by the questionnaire judges towards the stimulus photographs. These components were found in Section II, items $24,26,28,30,32,34$, and 36 (a total of seven items). The items appeared in the same format on the questionnaire as the instrumental component.

Interpersonal components of physical attractiveness items were taken from a pre-exisiting semantic differential scales (Petersen, 1976) and modified to fit the design of the questionnaire.

\section{Affect}

Affect components on the questionnaire referred to the absolute measuring of "liking." In other words, the affective scale measured how much the questionnaire judges or subjects would like or dislike the person in the photograph.

Pre-existing scales on the Affect component did not measure it absolutely but rather indirectly such as "I would like to go to a movie with you." Obviously, this item did not measure affect directly, as does, for example, the phrase "I like you," which does not leave any doubt of how one person feels towards another. For this reason, the investigator created a list of synonyms for expressing "liking" (affect). Affective items can be found in the 
questionnaire, Section III, items $39,42,45,48,51,54,57,60,63$, $66,69,72,75,78,81,84,87$, and 90 (a total of 18 items).

These items were phrased on the questionnaire, "I think that I would personally the stimulus," was followed by a seven point scale labeled, "Strongly Agree (7)" to "Strongly Disagree (1)."

Behavior

Behavior Predisposition. Behavior Predisposition measured the intent of the questionnaire judges' or subjects' behavior towards the stimulus photograph. These items on the questionnaire (see Appendix D) can be found in Section III, items $37,38,40,41,43,44$, $46,47,49,50,52,53,55,56,58,59,61,62,64,65,67,68,70$, $71,73,74,76,77,79,80,82,83,85,86,88,89,91,92,93$ and 94 ( a total of 40 items). These $i$ tems represented a variety of self-perceptions about communicative tendencies and different ways people feel about each other. The question format occurred in the same manner for the Behavior Predisposition as it did for the Affect items.

Items $37,43,44,46,47,50,55,59,64,67,70,77,80$ and 85 on the questionnaire were modified to fit this research from Kidwell and Both (1977). Kidwell and Both had tested and retested these items in their questionnaire to a population sample of adults ranging from 18 to 85 years old, across age groups.

Items $38,40,41,49,52,53,56,58,61,62,65,68,71,73$, $74,75,79,82,83,86,88,89,91,92,93$ and 94 on the OFIQ were 
modified items from Predisposition toward Verbal Behavior, Mortensen, Arnston and Lustig (1977). The items on this questionnaire had already been tested and retested with undergraduate students.

\section{Extraneous Items}

The fourth section of the questionnaire included a combination of items from the components of Instrumental Qualities, Interpersonal Qualities, and Affect. Instrumental items in this section of the questionnaire were items 96,98 and 102. Five Interpersonal items appeared in this part of the questionnaire. They were 95, 97, 99, $100,101,104$ and 105 .

Presentation of items on the questionnaire. Physical attribute items were presented in an order so that not one similar characteristic appeared together one right after another. For example, an item that followed "eyes" was not one like "eyebrows" but rather "hair." In this manner, the questionnaire judges were forced to cognitively evaluate the stimulus photographs without automatically responding with a set response.

Instrumental-Interpersonal items were randomized separately and then reordered in this pattern: Instrumental/Interpersonal/ Instrumental.

Affect and Behavior Predisposition items were presented in the order of every two items on Behavior Predisposition to every one item on Affect through the end of the section. Again, similar items were separate so that an automatic response would not occur. 
Miscellaneous items on Instrumental, Interpersonal and Affect components were included at the end of the questionnaire and included those items taken from the content analyses of the tape-recorded responses from selection judges (see Appendix G).

The Instrumental and Interpersonal items not included in the Miscellaneous Section already had been tested and retested by Petersen across age groups.

Section IV contains items derived from the selection judges' tape-recorded responses. These items were put at the end of the questionnaire to differentiate them from items selected from Petersen's study.

Creating a Matrix with Physical Attractiveness Intensity Levels for the Stimulus Photographs

Physical attractiveness was a controlled variable by assigning the stimulus photograph one of three intensity levels: high, medium or low. Based upon Section II statistical analyses, the intensity levels had already been established. This was based upon the means and the larger standard deviations.

A mathematical matrix was designed with which each judge evaluated the stimulus photographs so that the same intensity levels did not occur consecutively.

The number assigned to each photograph was (3) high, (2) medium, or (1) low based upon the descriptive statistics in Phase II.

Photo orders were created to account for any possible order effect that might have changed the questionnaire judges' way of responding to the photographs. An "order effect" is seen by the 
investigator as the way in which an evaluating participant may respond to the serial presentation of stimulus photographs. For example, if a questionnaire judge looks first at an older person and then at a younger person in a photograph, it may effect how he/she evaluates the latter's characteristics towards the positive. Whereas, if the same questionnaire judge looks first at a younger person and then at an older person in a picture, it may effect the judge's response towards the negative. Encompassing all possible combinations of photographs in all three age groups and physical attractiveness intensity levels may help to eliminate this kind of bias.

Figure 6 shows the three photo orders with the intensity levels of the stimulus photographs given to the questionnaire judges.

\section{STIMULUS PHOTOGRAPHS}

\begin{tabular}{ccc}
\hline $\begin{array}{l}\text { PHOTO } \\
\text { ORDER 1 }\end{array}$ & $\begin{array}{c}\text { PHOTO } \\
\text { ORDER 2 }\end{array}$ & $\begin{array}{c}\text { PHOTO } \\
\text { ORDER 3 }\end{array}$ \\
\hline 1 & 2 & 3 \\
2 & 3 & 2 \\
3 & 1 & 1 \\
1 & 2 & 3 \\
2 & 3 & 2 \\
3 & 1 & 1
\end{tabular}

Number of stimuli with intensity levels within each Photo Order, $\mathrm{N}=6$. Total Number of stimuli, included in all three Photo Orders, $N=18$.

Figure 6. Matrix representing stimulus photographs physical attractiveness intensity level: (3) high, (2) medium and (1) low. 
Presentation of Stimulus Photographs to Questionnaire Judges

Three photo orders were presented to the questionnaire judges. Each questionnaire judge was presented with one age group of stimulus photographs (Young, Middle-Aged or $01 \mathrm{~d}$ ). Judges were presented with the same photo order at the test and retest of the items on the questionnaire. There was a two-week time lapse in between judges' evaluations to determine the internal reliability of the items on the questionnaire.

A total of 18 photographs were used in this phase of the study with six pictures in each age group (Young, Middle-Aged and 01d). Three males and three females served as the stimuli to each age group, creating a total of six pictures that each questionnaire judge evaluated.

Overview of Data Analyses Used to Reduce the Number of Items on the Questionnaire

The five components defining physical attractiveness were independently assessed. A pool of 105 items was initially generated on the Original First Impression Questionnaire (referred to hereafter as (FIQ). Based upon the data analyses below, the number of items on the questionnaire was reduced to 30 . This new version was called Modified First Impression Questionnaire (known as MFIQ). A copy of both questionnaires, OFIQ and MFIQ can be found in Appendix $D$. Figure 7 , on the following page, shows the total number of items within each component used on the Original and Modified versions of the questionnaire. 


\begin{tabular}{|c|c|c|c|}
\hline Component & Section & Identification & $\begin{array}{l}\text { Total No. } \\
\text { of Items }\end{array}$ \\
\hline Physical Attributes & 1 & $\begin{array}{l}1^{\star}, 2,3,4,5^{\star}, 6^{\star} \\
7,8,9^{\star}, 10,11,12^{\star} \\
13,14,15^{\star}, 16, * 17 \\
18^{\star}, 19,20,21^{\star}, 22^{\star}\end{array}$ & 22 \\
\hline \multirow[t]{2}{*}{ Instrumental Qualities } & 2 & $\begin{array}{l}23,25^{\star}, 27^{\star}, 29,31^{\star} \\
33^{\star}, 35^{\star}\end{array}$ & \\
\hline & 4 & $96,98,102$ & 10 \\
\hline \multirow[t]{2}{*}{ Interpersonal Qualities } & 2 & $\begin{array}{l}24^{\star}, 26,28^{\star}, 30,32^{\star} \\
34^{\star}, 36^{\star}\end{array}$ & \\
\hline & 4 & $\begin{array}{l}95,97,99,100,101 \\
104,105\end{array}$ & 14 \\
\hline \multirow[t]{2}{*}{ Affect } & 3 & $\begin{array}{l}39,42^{\star}, 45^{\star}, 48,51, \\
54^{\star}, 57^{*}, 60^{\star}, 63,66, \\
69,72,75,78,81,84 \\
87,90^{\star}\end{array}$ & \\
\hline & 4 & 103 & 19 \\
\hline $\begin{array}{l}\text { Behavior } \\
\text { Predisposition }\end{array}$ & 3 & $\begin{array}{l}37^{\star}, 38,40,41,43, \\
44^{\star}, 46,47,49,50 \\
52,53,55,56,58,59 \star \\
61,62,64,65,67 \star, 68, \\
70,71,73,74,76,77^{\star}, \\
79,80,82,83,85,86, \\
88,89,91,92,93,94\end{array}$ & , \\
\hline
\end{tabular}

Number of items, $\mathrm{N}=105$

*The asterisk symbol indicates the selected items used on the Modified First Impression Questionnaire (MFIQ). $(O F I Q)$.

Figure 7. Items on the Original First Impression Questionnaire 
The statistics analyzed by the Honeywell 66/40 computer using the Statistical Package for Social Scientist (Nie, Hull, Jenkins, Steinbrenner and Bent, 1970) can be found in Appendix E. For the purposes of this series of analyses, all variables were left in their original state. The Statistical analyses used in paring down the number of items on the questionnaire were: descriptive statistics, reliabilities, factor analysis and Pearson $r$ correlation. For a more detailed computer statistical outline of the "unit of analyses" in reducing the number of $i$ tems on the questionnaire, refer to Appendix $E$.

After the 105 items on the OFIQ were reduced to 30 items on the Modified version, the questionnaire was again analyzed statistical1y. The statistics used on the MFIQ were descriptive statistics, reliabilities and factor analyses.

\section{DESCRIPTIVE STATISTICS}

Tables I through $V$ provide summaries of the means and standard deviations of the OFIQ. All items are included under one each of the five components: (1) Physical Attributes, (2) Instrumental

Qualities, (3) Interpersonal Qualities, (4) Affect and (5) Behavior Predisposition.

Observations totalled 144 , consisting of 72 test and 72 retest. When an asterisk appears beside a given item on any table, it indicates that the item was selected for the MFIQ. 
The data gathered from the interviews were analyzed with test, retest and total tabulation across variables of age and sex of stimulus photographs. The standard deviations of the total (combination of test and retest) were examined separately to select only those items with a large standard deviation score, to allow for more variability in opinion.

If judgements were normally distributed about the "true value", the end category distributions were curtailed, thus displacing the mean values toward the middle of the category range. Items at the means at either end of the continuum were eliminated. The items finally chosen were selected because they fell within all age groups.

\section{Physical Attributes}

Table I illustrates the means and standard deviation computation for the test, retest and total. Physical Attribute Items 1 through 22 reveal that all the items had fairly large standard deviations. Out of the 22 items in this component, the items with standard deviations of 1.39 to 1.66 were potential items for the final questionnaire. Those ten selected items to be used on the modified questionnaire were numbers $1,5,6,12,15,16,18,20,21$, 22. It can be seen by inspecting Table I that these selected items may have had comparable means to other items on the table. However, their standard deviations were larger, allowing more variability in opinion. 
TABLE I

TEST-RETEST DESCRIPTIVE STATISTICS ON

PHYSICAL ATTRIBUTES ITEMS (OF IQ)

\begin{tabular}{|c|c|c|c|c|c|c|}
\hline & \multicolumn{2}{|c|}{ Test } & \multicolumn{2}{|r|}{ Retest } & \multicolumn{2}{|c|}{ Total 1} \\
\hline Item & Mean & $\begin{array}{l}\text { Standard } \\
\text { Deviation }\end{array}$ & Mean & $\begin{array}{l}\text { Standard } \\
\text { Deviation }\end{array}$ & Mean & $\begin{array}{l}\text { Standard } \\
\text { Deviation }\end{array}$ \\
\hline
\end{tabular}

Section I

*1. Physical

Appearance

4.25

1.46

4.23

1.57

4.24

1.51

2. Nose

4.43

1.28

4.48

1.30

4.53

1.28

3. Face Shape

4.54

1.24

4.45

1.40

4.50

1.32

4. Eyebrows

4.47

1.39

4.40

1.41

4.44

1.40

*5. Facial Grooming

*6. Smile

4.79

1.39

4.68

4.56

1.52

7. Weight

4.18

1.22

8. Teeth

4.64

*9. Hairstyle

3.94

1.42

4.47

1.53

4.74

1.46

4.14

1.57

4.52

1.54

4.40

1.20

4.16

1.63

3.99

1.46

4.52

1.21

10. Personal

11. Chin

Hygiene

4.93

1.28

4.76

1.50

3.96

1.44

1.56

*12. Eyes

4.62

1.25

4.50

1.36

4.84

1.32

13. Posture

4.75

1.45

4.64

1.21

4.56

1.22

14. Mouth

4.52

1.19

4.38

1.53

4.69

1.48

*15. Facial

4.53

1.39

4.36

1.06

4.45

1.13

1.44

4.45

1.42

Expression

$4.72 \quad 1.52$

4.52

1.66

4.62

1.59

$\star 16$. Skin

4.72

1.44

4.53

1.54

4.63

1.49

17. Ears

4.39

1.46

4.36

1.33

4.38

1.39

18. Health

4.75

1.48

4.46

1.44

4.61

1.46

19. Neck

4.57

1.23

4.39

1.24

4.49

1.23

20. Hair

4.34

1.66

4.26

1.44

4.30

1.56

Groomed

5.06

1.49

4.88

1.48

4.97

1.48

22. Very Phy.

Attractive

Very Phy.

Unattractive

4.39

$.41 \quad 4.42$

1.56

4.41

1.48

Number of total observations, $N=144$, each test/retest consisted of $\mathrm{N}=72$.

*The asterisk symbol indicates the selected items used on the Modified First Impression Questionnaire (MFIQ).

Note: Each mean and standard deviation was rounded off to the nearest hundredth. 
Cognitive

Instrumental Qualities. Table II shows the mean "overall impression" on the Instrumental Qualities component found in Section II, items $23,25,27,29,31,33,35$ and in Section IV, items 96,98 and 102 (a total of ten items). The standard deviation of the Instrumental Qualities component was slightly smaller than that of the Physical Attribute component. Items with a standard deviation of 1.16 to 1.39 were selected as potential items. Items $25,27,31,33$ and 35 (a total of five items) were selected to be used on the MFIQ. Interpersonal Qualities. Table III, shows the means and standard deviations of the test, retest, and total items on the Interpersonal Qualities component. The items categorized as Interpersonal Behavior were found in Section II, items $24,26,28$, $30,32,34,36$ and in Section IV, items 95, 97, 99, 100, 101, 104 and 105 on the OFIQ. These means and standard deviations were comparable to the Instrumental Qualities component. Still, the Physical Attribute component in general had larger standard deviations and means. As previously stated, the larger standard deviations $(1.11$ to 1.43) were the "priority" items picked for potential use. Items with extreme means were not selected. The selected items for the Interpersonal Qualities component were $24,28,32,34$ and 36 (a total of five items) on the Modified questionnaire. 


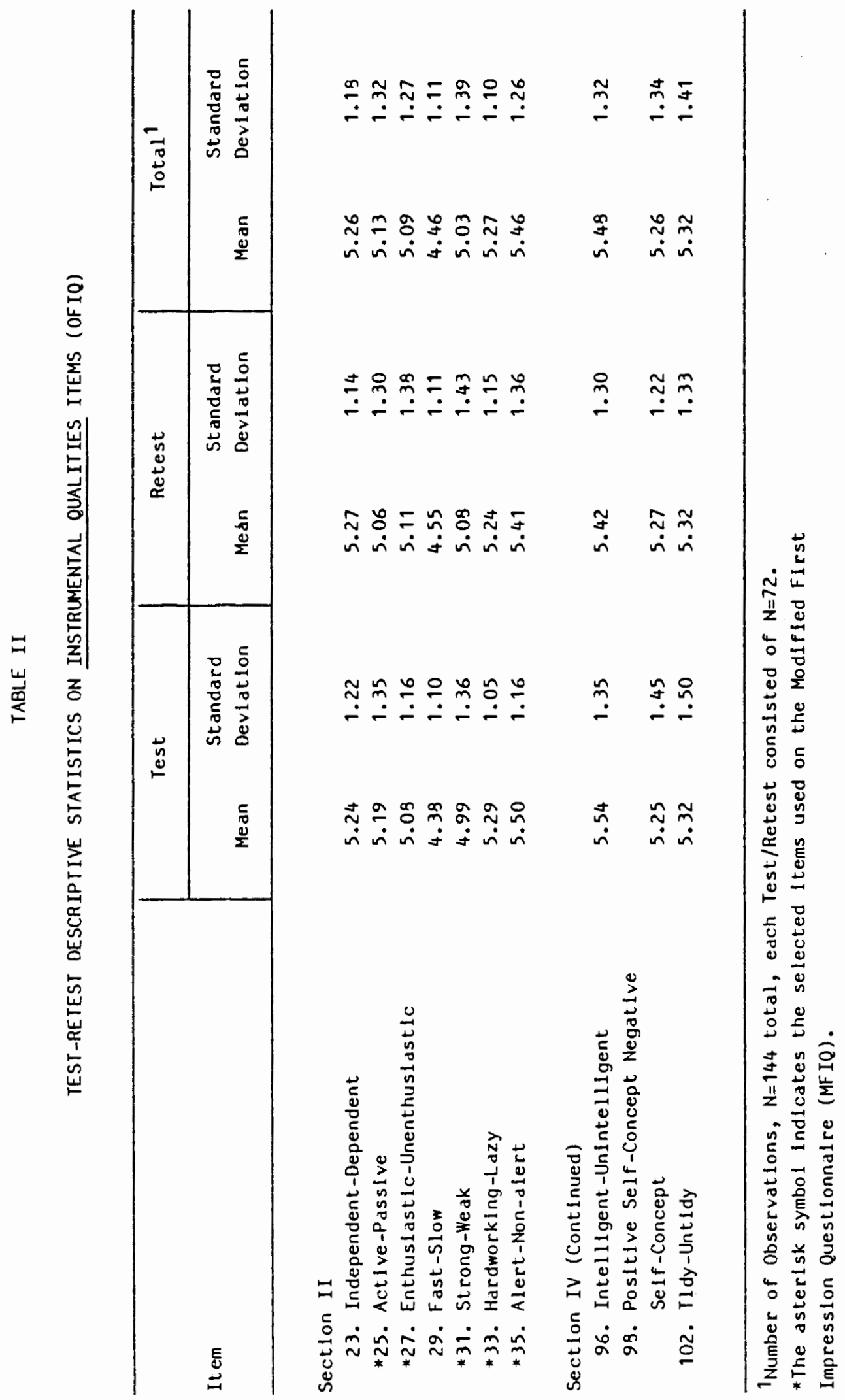




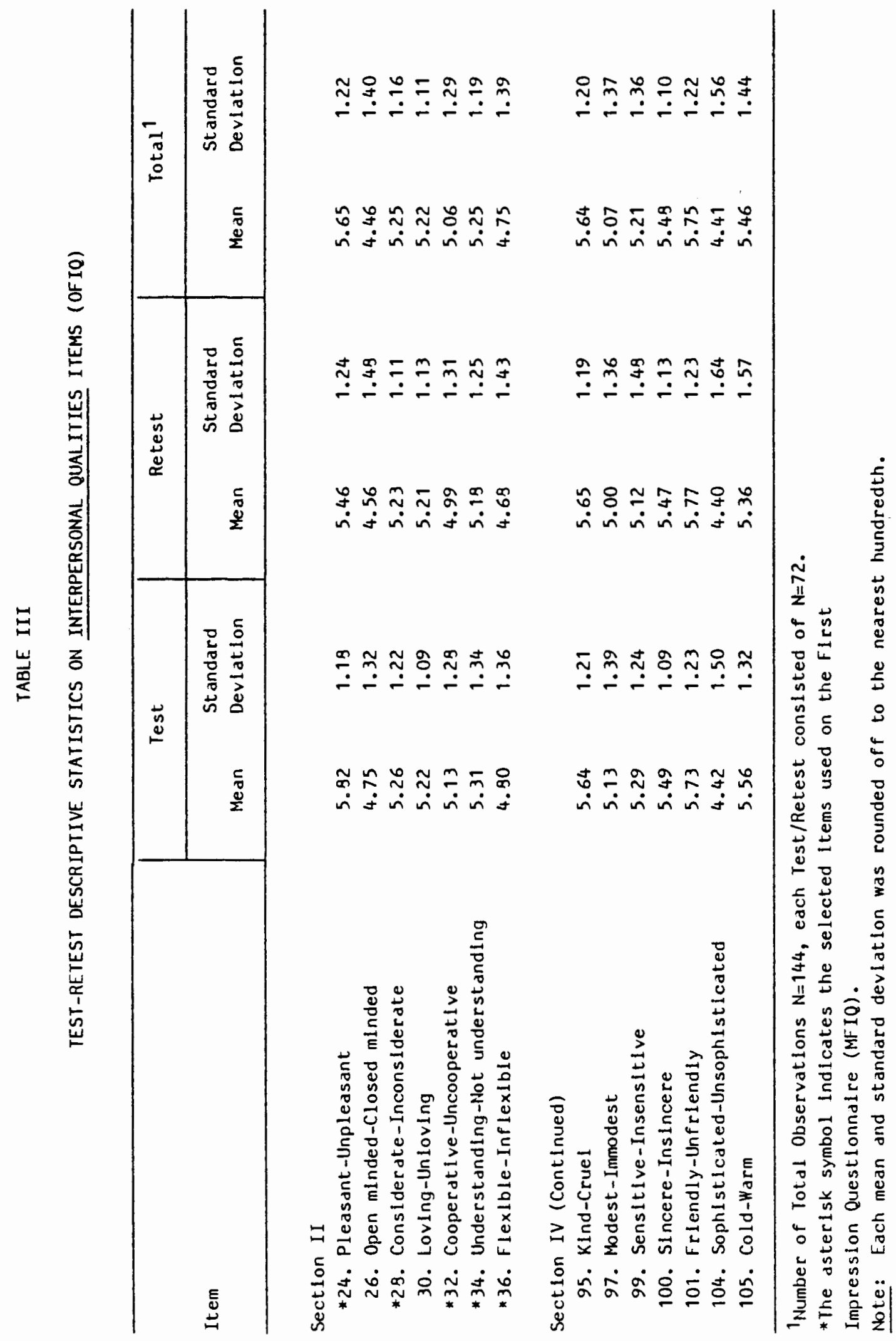




\section{Affect}

Table IV shows the Affect component consisting of test, retest and total mean and standard deviations. The 19 Affect items can be found in Section II I on the Questionnaire, numbered $39,42,45,48$, $51,54,57,60,63,66,69,72,75,78,81,84,87,90$ and in Section IV, numbered 103.

Items with standard deviations of 1.44 to 1.76 were chosen as potential items to be used on the MFIQ. The six items used on the MFIQ were $42,45,54,60,66$ and 69.

Generally speaking, Affect items had consistently larger standard deviations than did the Physical Attribute and Behavior Predisposition component items. The other two components, Instrumental and Interpersonal Qualities, had smaller standard deviations indicating a greater consensus of opinion on any given item. The means on the Affect component were equivalent to the Physical Attributes, Instrumental Qualities, and Interpersonal Qualities.

Behavior

Behavior Predisposition. Behavior Predisposition items can be seen on Table V. The items can be found in Section III on the OFIQ numbered $37,38,40,41,43,44,46,47,49,50,52,53,55,56,58$, $59,61,62,64,65,67,68,70,71,73,74,76,77,79,80,82,83$, $85,86,88,89,91,92,93$, and 94 (a total of 40 items). 


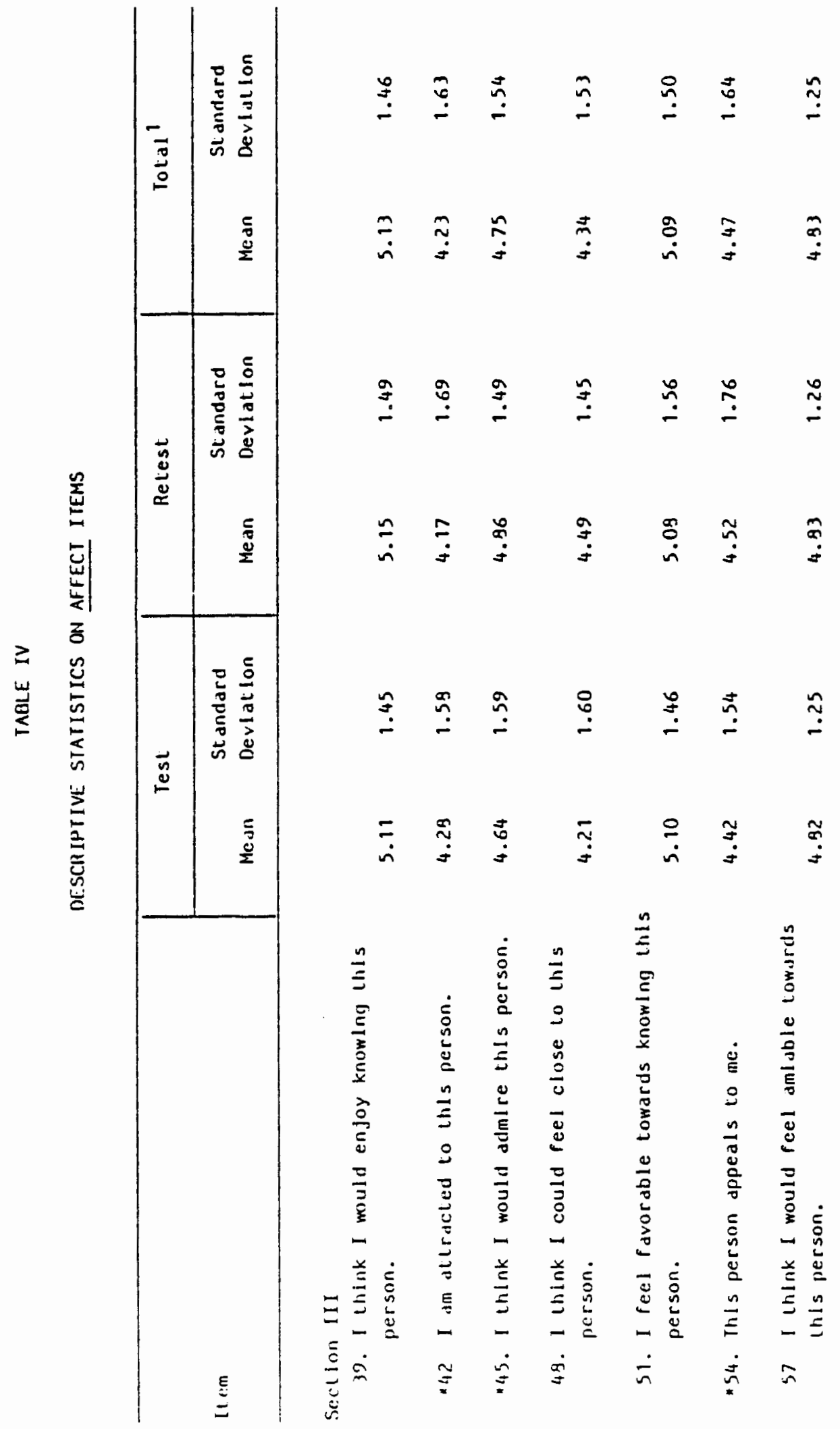




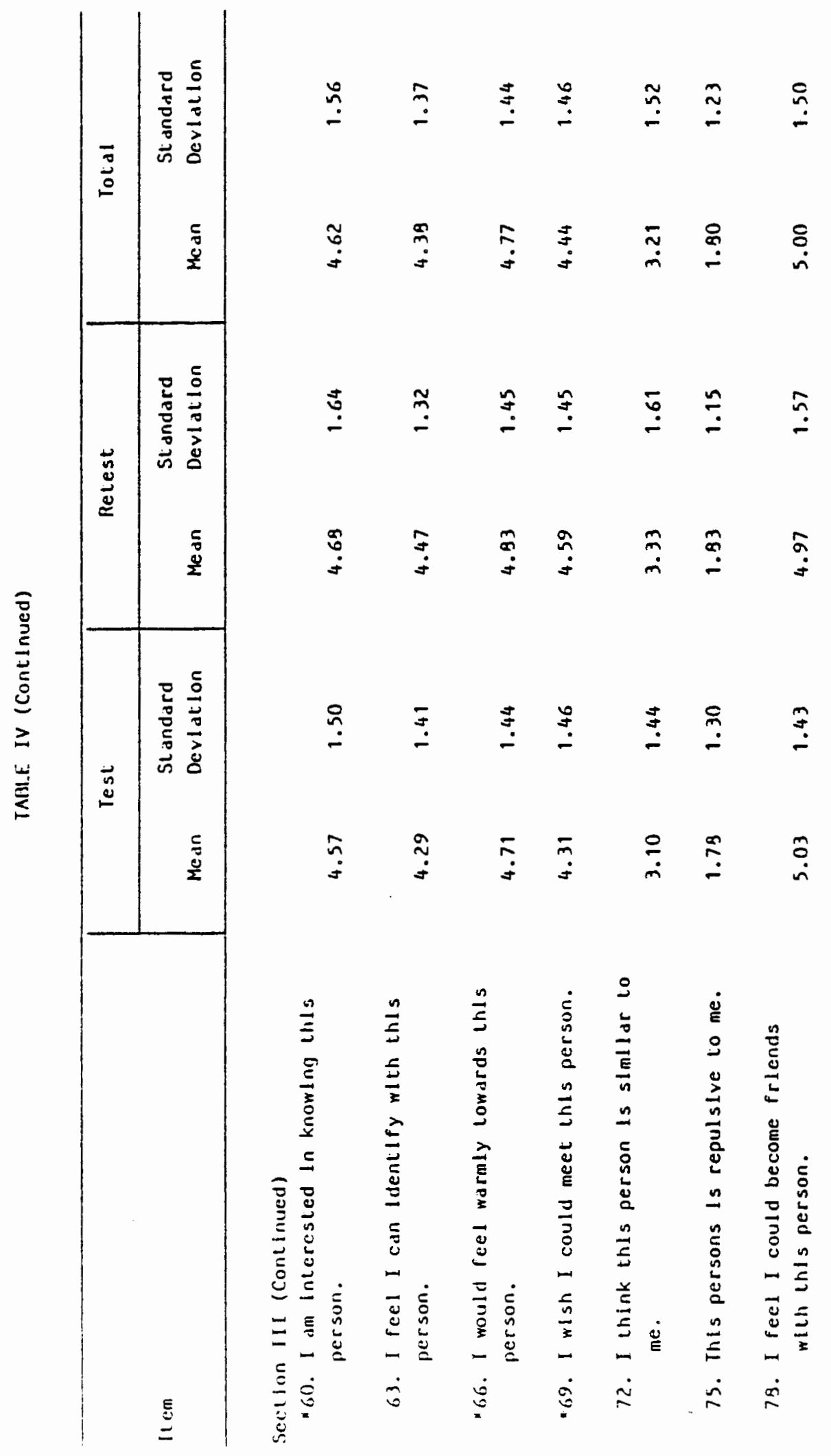




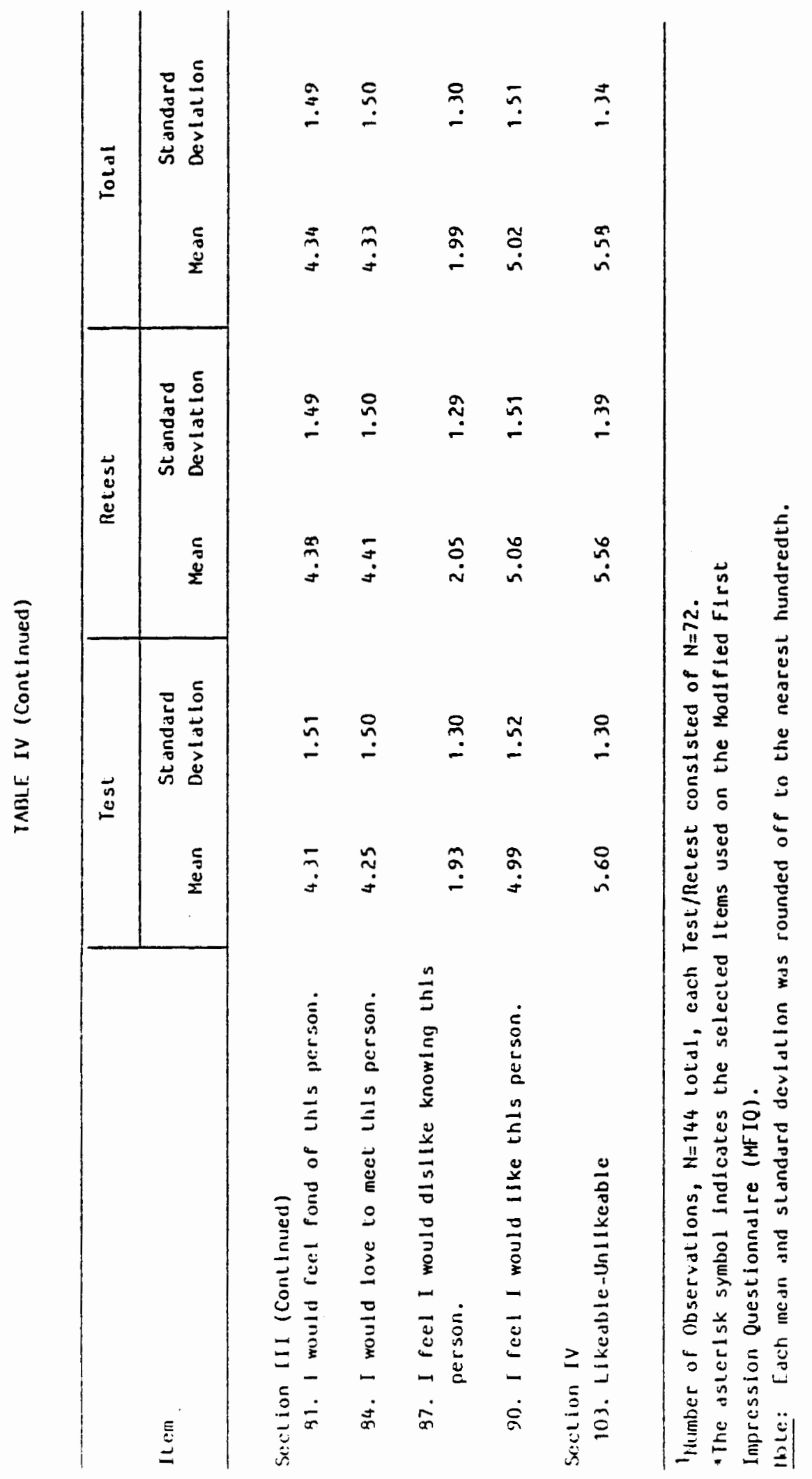




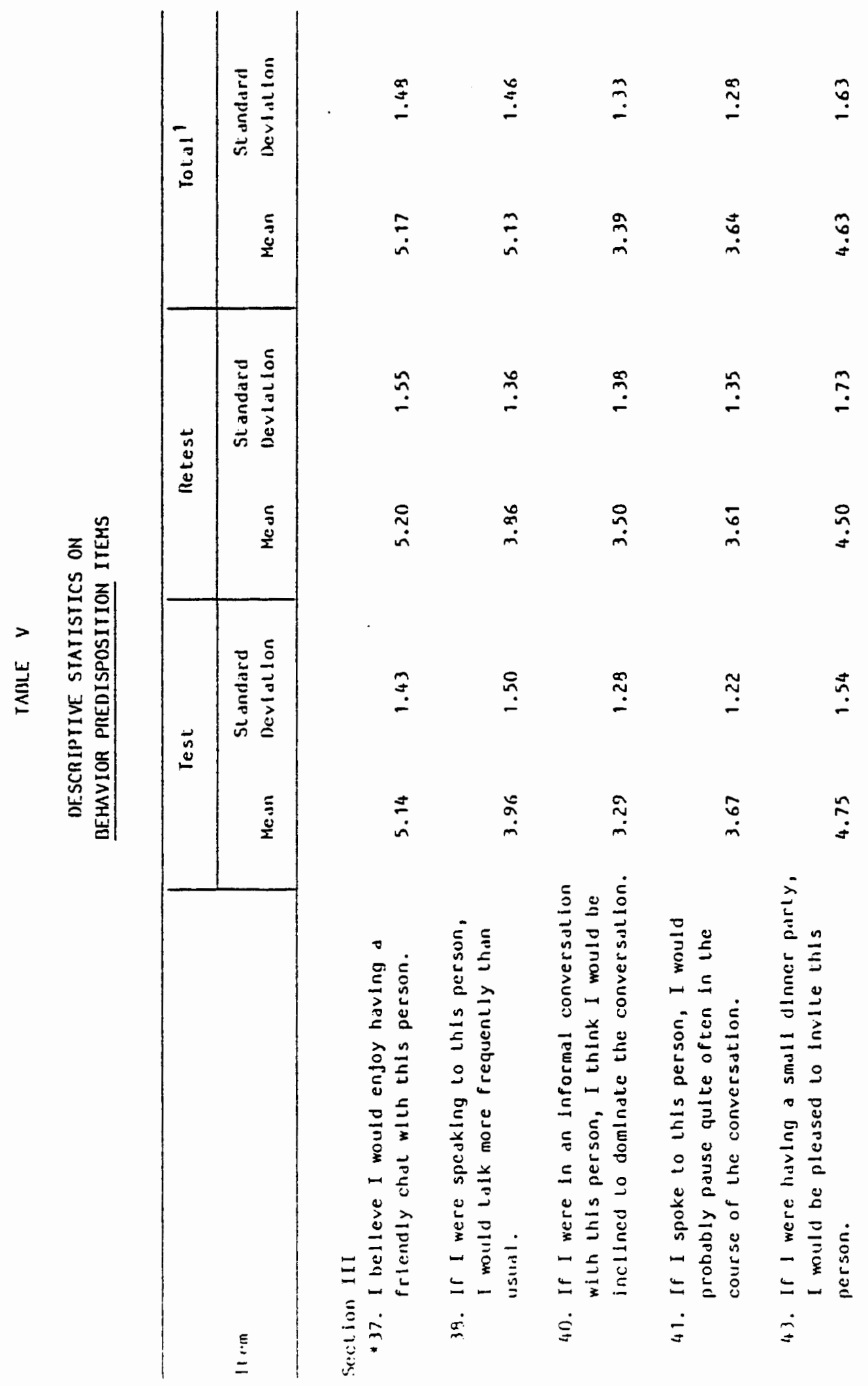




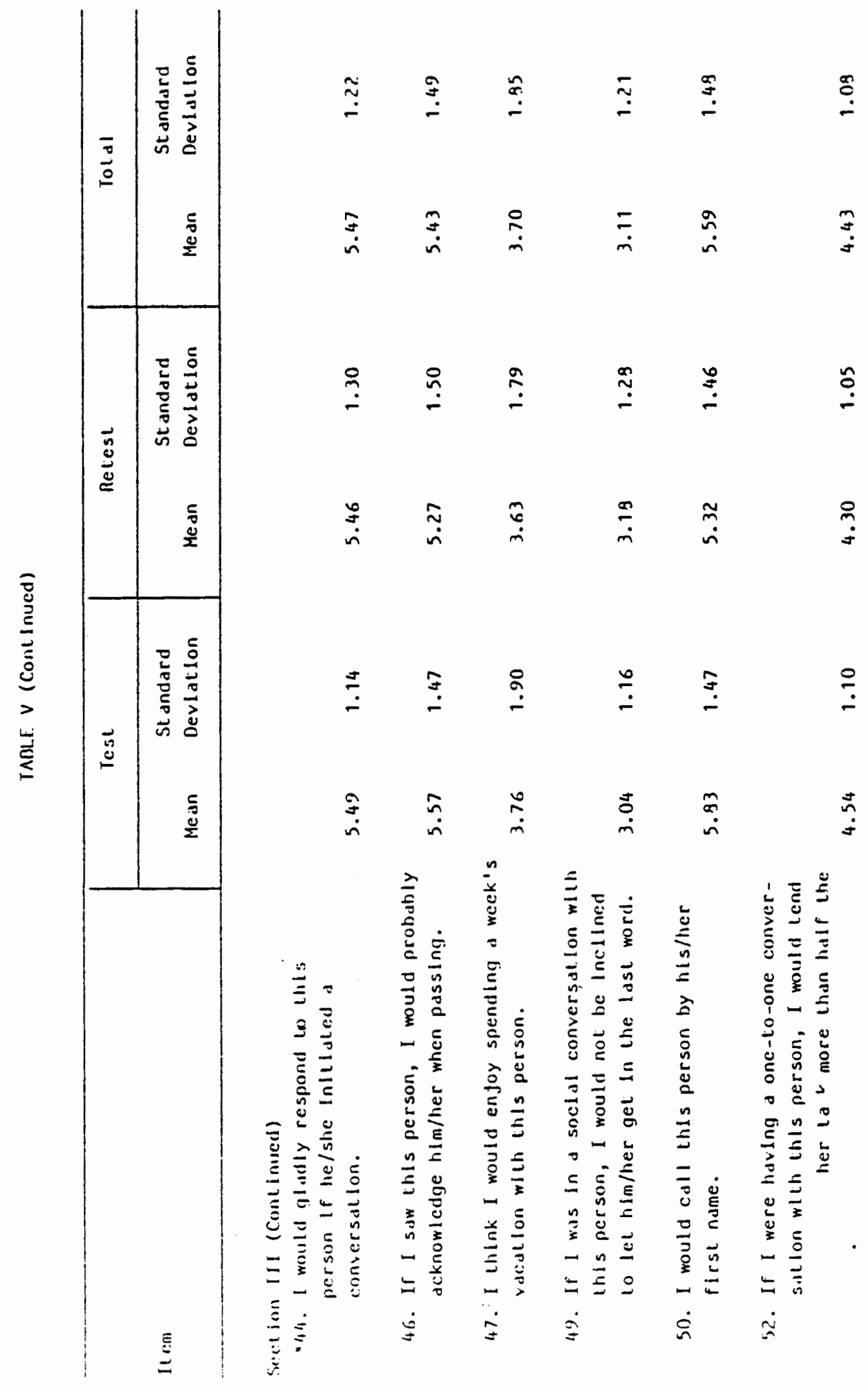




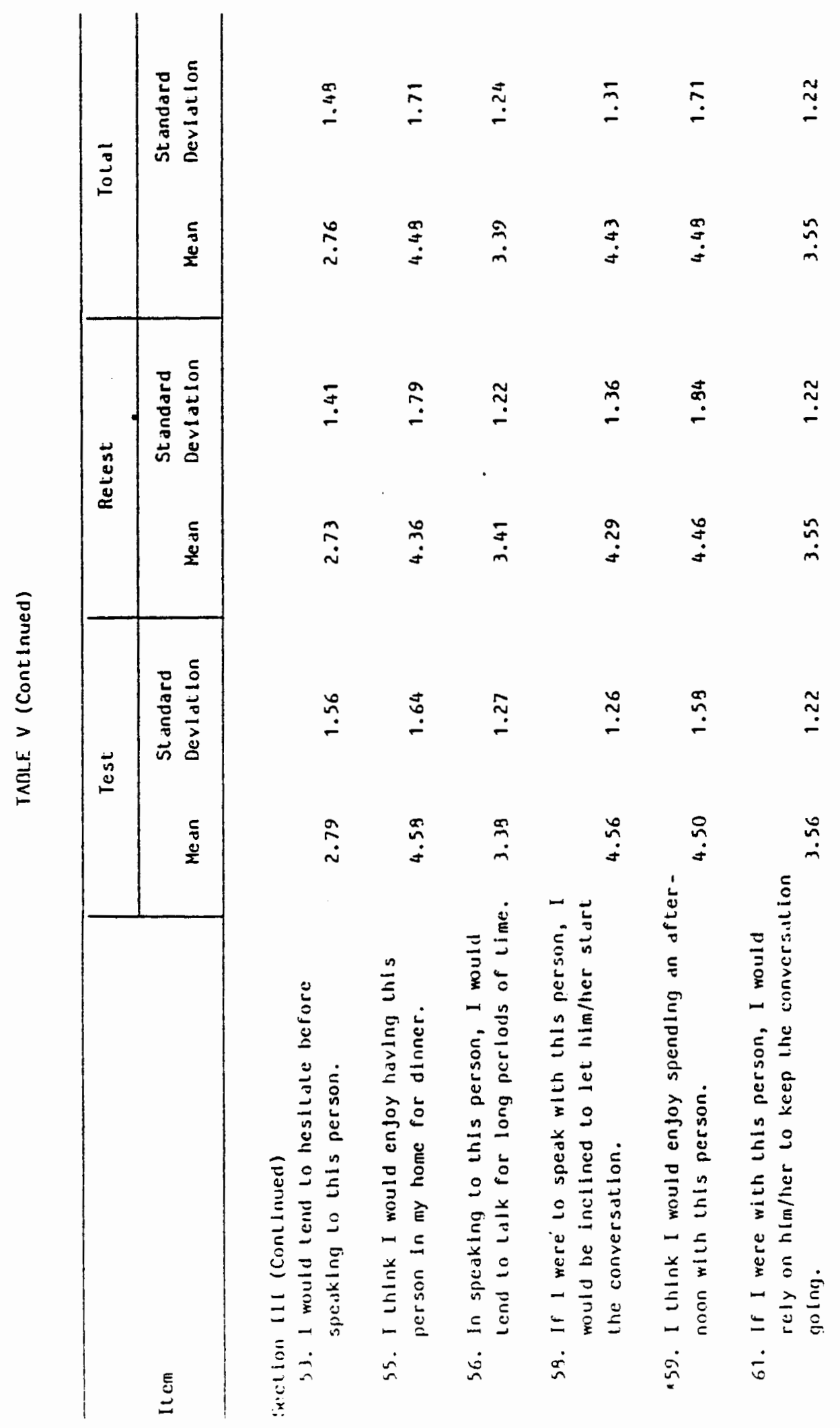




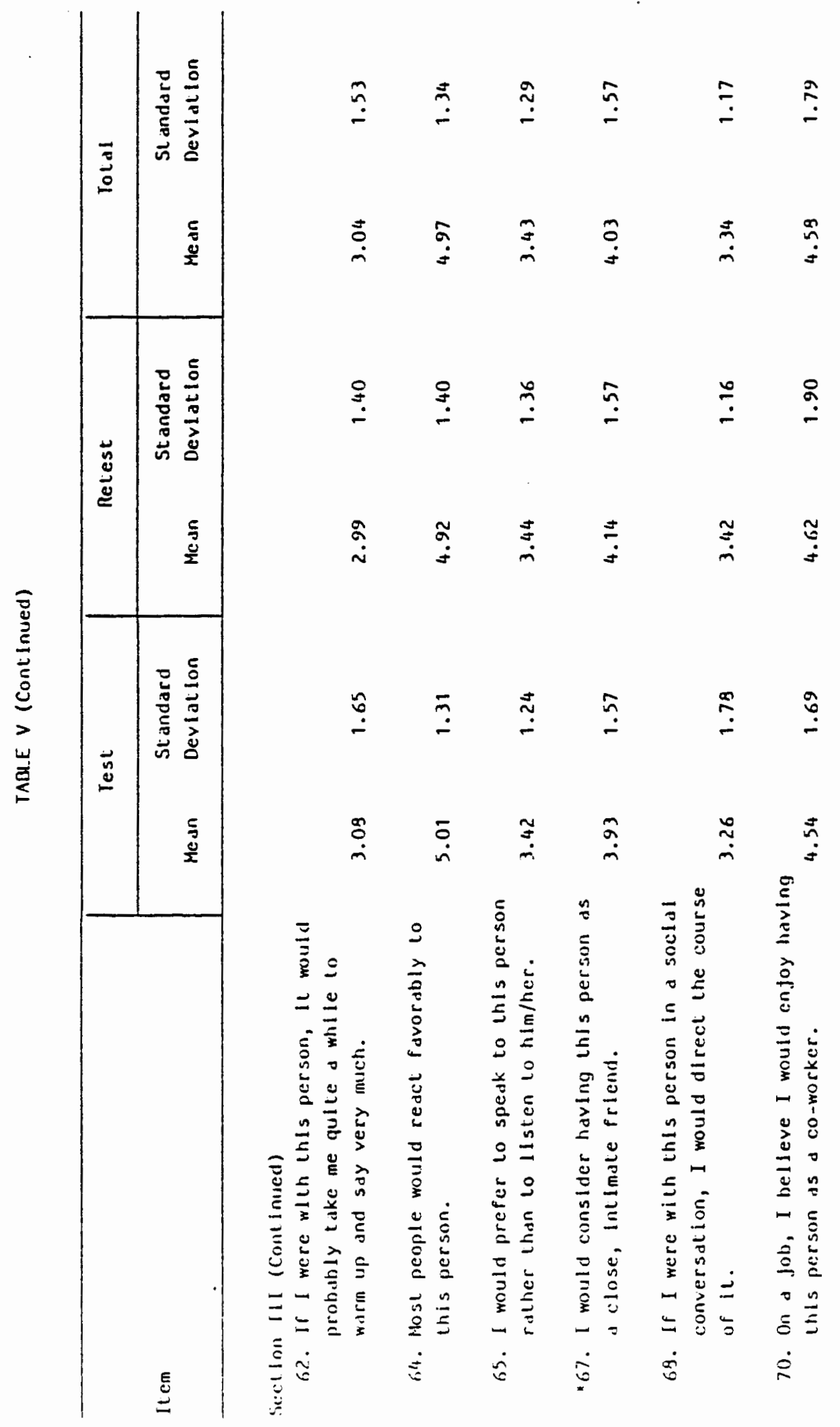




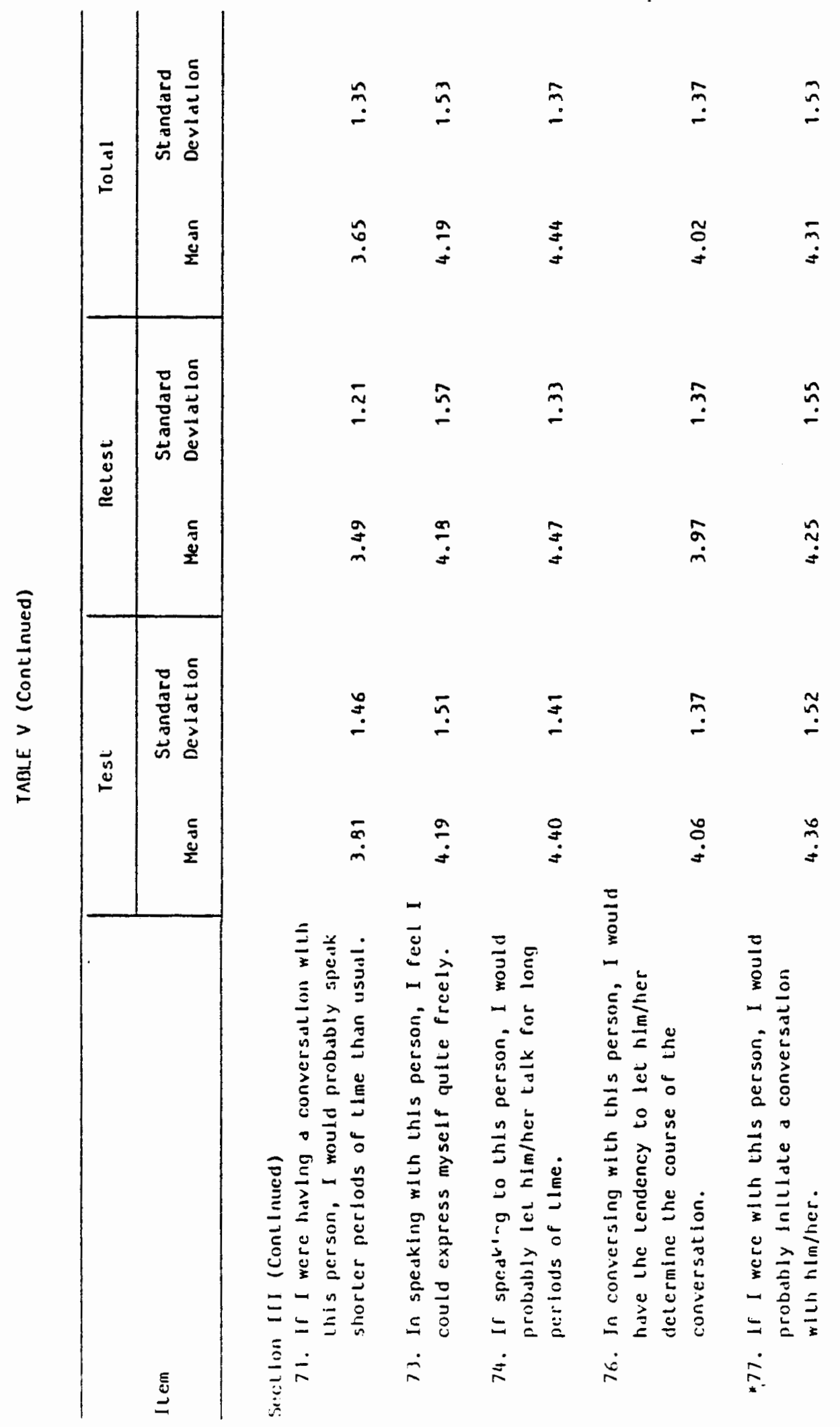




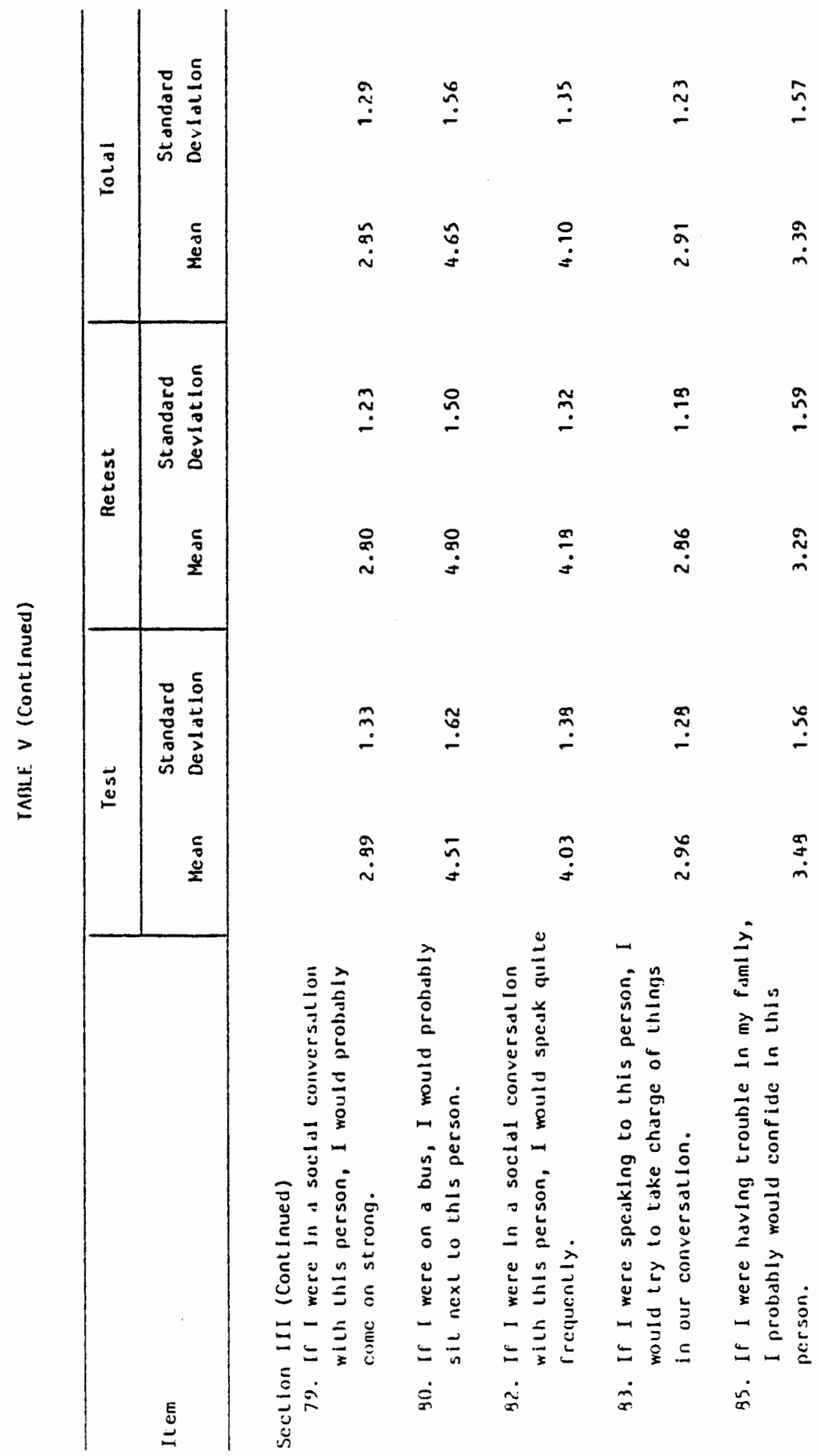




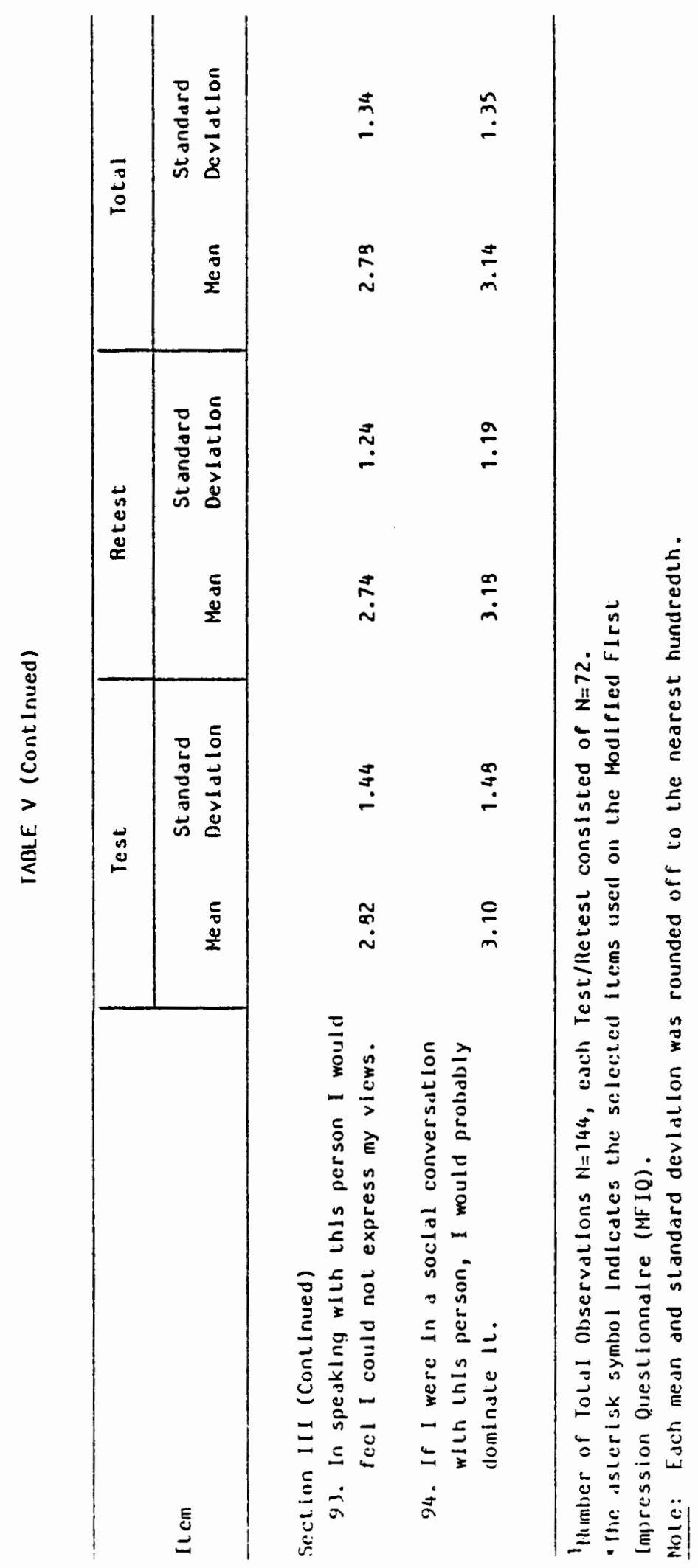


As seen on Table $V$, the means do not appear within a consistent range but fluctuate either more towards "Strongly Agree" or "Strongly Disagree." The five items selected for potential use on the MFIQ ranged with a standard deviation of 1.14 to 1.84. Selected items were number $37,44,59,67$ and 77 .

It appears that the other components had means that fell generally within the neutral range. Behavior Predisposition standard deviation computations were generally larger than the Physical Attribute and Affect components indicating more variability in opinion.

\section{FACTOR ANALYSES}

Table VI through IX provides a summary of the factor analyses done on the OFIQ. As indicated on the table, the items were included under one of the five components.

Factor analysis was another test used on the OFIQ which contributed to the final reduction of 105 items to the 30 items on the final, Modified version. The PA 1 factoring method (principal without iteration) was used on the five components defining physical attractiveness. In this method, as described in the Statistical Package for Social Sciences (Nie et al., 1970), the main diagonal of the correlation matrix that extracts exact mathematical transformation of original variables was not altered. PA 1 does not require any assumptions about the general structure of the variables. As indicated on Table VI, Rotated Factor Matrix on Physical Attribute 
Items, the column entries are called "loadings" and interpreted like correlation coefficients (i.e, the loading range from -1.0 to +1.0 ), indicating the degree and direction of association of the components with the factors. For example, Physical Appearance on Factor $A$ is -.86 and the association between the Physical Attribute and Factor A is negative. The percent of the variance of the item shared with a Factor is equal to the squared factor loading multiplied by 100 . In other words, the percent of the variance of Physical Appearance held in common with Factor $A$ is equal to $(-.822)(100)$ or 74 percent.

The common factor variance of a scale is called its "communality." The communality of a scale equals the sum of its squared factor loadings. In other words, the communality in the Physical Appearance example is equal to $(-.862)+(-.162)+(-.052)+(-.062)$ or .77 . This means that 77 percent of the variance of the item, "Physical Appearance" has been accounted for by the four factors, leaving 23 percent of the variance unaccounted for by the common factors.

Tables VI through IX reflect the factor analysis done on the five components determining physical attractiveness. Items with the largest loadings were considered "potential" items to be used on the MFIQ al though other computer statistics were considered in the selection process. On all the tables, the asterisk symbol beside the item number indicates the selected items used on the modified version of the questionnaire. The brackets on the tables indicate if the item has a factor loading greater than or equal to .50 . 


\section{Physical Attributes}

Twenty of the 22 Physical Attribute items had communalities with high correlations ranging from .64 to .82 . Only 18 of the items had loadings greater than .58 on one of the factors and less than -.38 on the remaining factors. Table VI contains the factor loadings for each of the 22 items on Physical Attributes. All but three of the items, 8, 13 and 17, had low loadings which may be explained by the inability of the judges to see and judge the stimulus photographs' teeth and posture. Again, the stimulus photographs did not show the weight, posture, teeth, ears, and so forth of their subjects.

Item 22, Very Physically Attractive to Very Physically Unattractive had the highest factor loading of .82. As stated earlier, this item has been used throughout the study.

\section{Cognitive}

Instrumental qualities. Table VII reflects the Rotated Factor Matrix on Instrumental items, showing all the items had loadings greater than or equal to .60 . Six of the ten items had factor loadings greater than .70 and four of the items had loadings for all the items indicating high factor loadings. Thus, all ten items were considered "good items" for the MFIQ. Other computed statistics were also taken into consideration to determine the selected items for the Modified version of the questionnaire. 


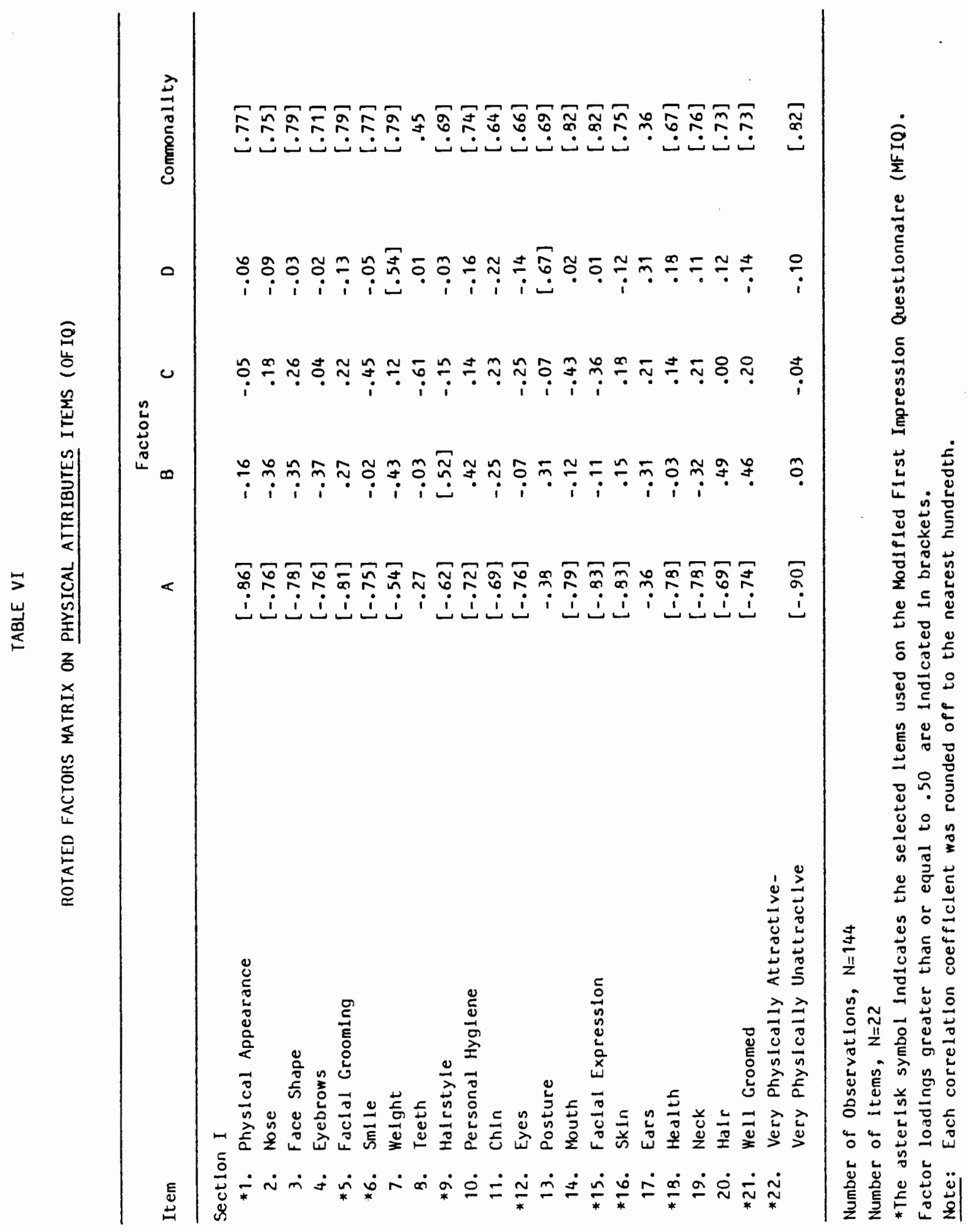




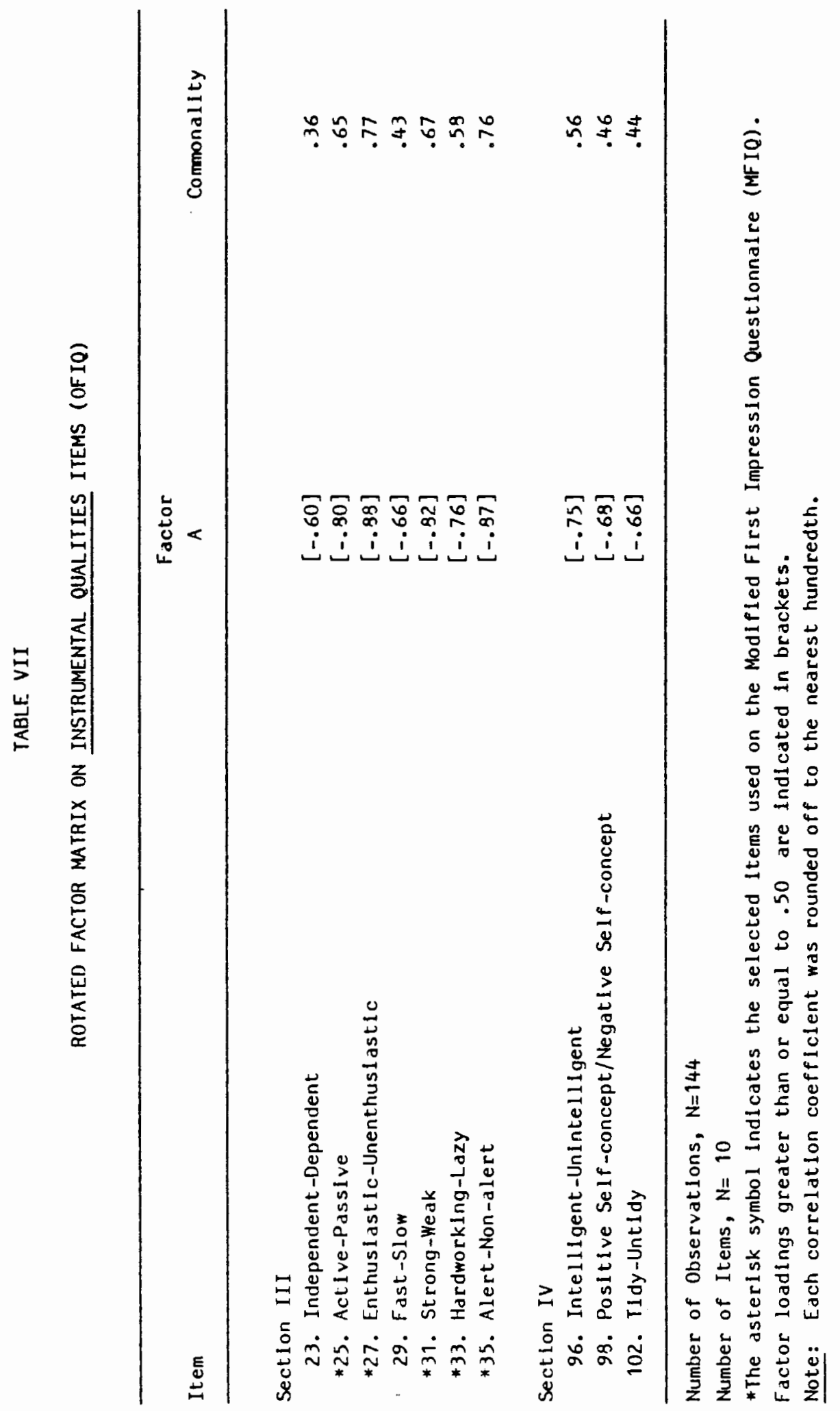


Seven of the ten Instrumental Qualities items had communalities with high correlations ranging from .56 to .77 . The lowest correlation on Table VII, Item 23, "Independent versus Dependent," was .36. This correlation was low because of the one-dimensional quality of the photographs used as stimulus materials. The low communality correlations may prove that a picture is not necessarily worth a thousand words.

Interpersonal Qualities. The Rotated Factor Matrix on Interpersonal Quality Items are shown in Table VIII. Thirteen of the 14 items on the Interpersonal component had factor loadings greater than or equal to .64 . Item 104, "Sophisticated versus Unsophisticated" had an extreme low factor loading of .35. This item may have been low because our culture has not defined a stereotype relationship between sophistication in facial appearance.

As indicated in Table VIII, all the communality correlations were high except item 104, "Sophisticated versus Unsophisticated," with a correlation of .36. Overall, the Interpersonal Qualities items reflect "high" factor loadings and communalities.

\section{Affect}

The Affect component consisted of 19 items with high factor loading and communalities, as seen in Table IX. Fifteen of the 19 items had high factor loadings greater than or equal to .67. The lowest loadings were items $63(.58), 72(.59)$, and $75(.58)$, considered moderately high correlations. 


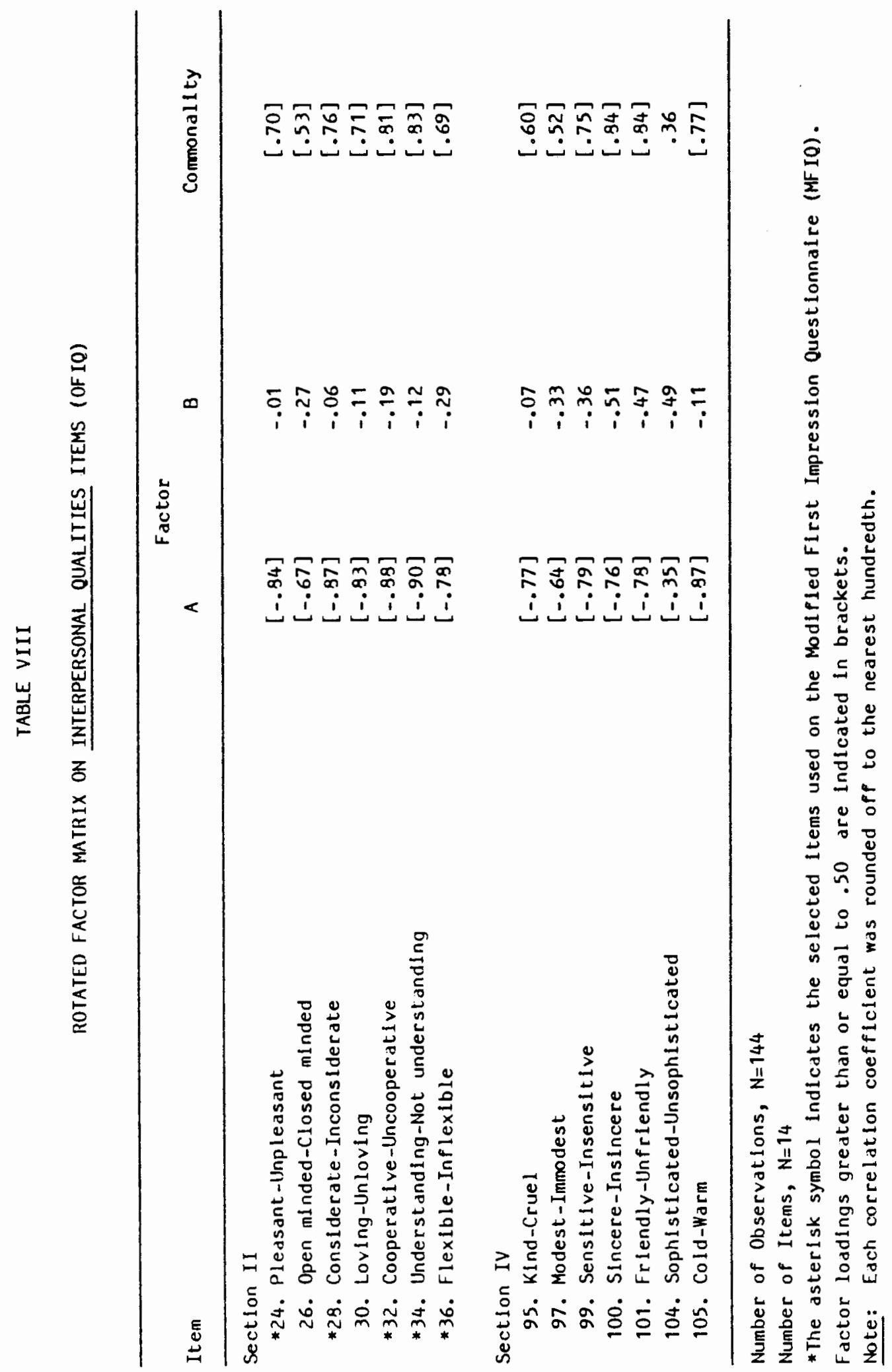




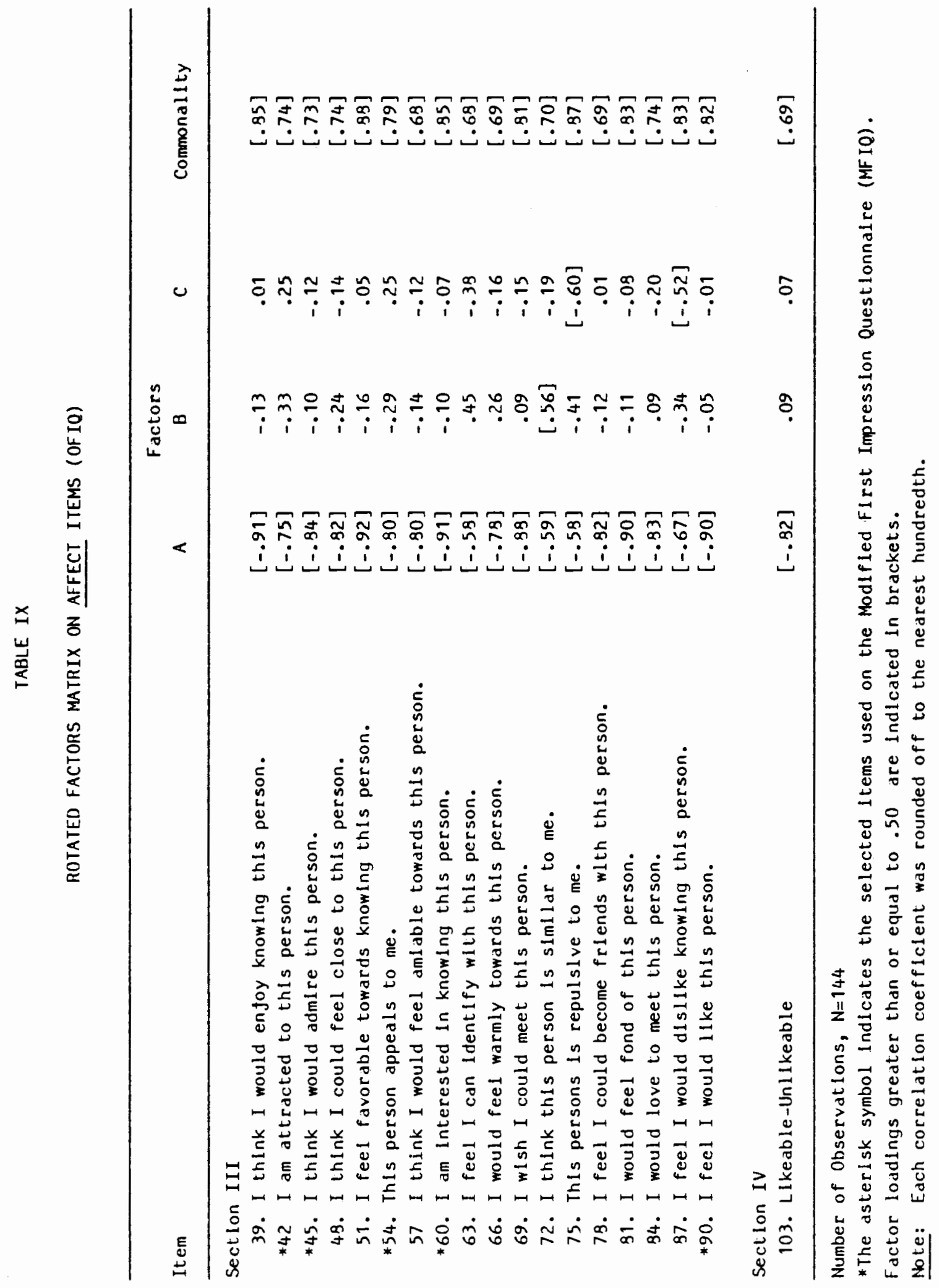


All of the items had high communalities correlations and were considered "potential" items to be used on the Modified questionnaire.

Behavior

Behavior predisposition. Table $X$ summarizes the Rotated Factors Matrix on Behavior Predisposition items. Twenty-three of the 40 items had factor loadings greater than or equal to .50. Item 37, "I believe I would enjoy having a friendly chat with this person" had a correlation of .90 .

All the communality correlations ranged from .54 to .85 . These items were considered moderately high to high correlations. Neither the factor loadings nor the communality correlations were consistently as high among the other items as were the four other components defining physical attractiveness (i.e., Physical Attributes, Instrumental Qualities, Interpersonal Qualities and Affect).

\section{PEARSON $r$ CORRELATION}

Pearson $r$ correlation coefficients determined the degree of association between the test and retest within each component defining physical attractiveness in this study. As such, it measured reliability, the "strength" of individual items, and thus contributed to the overall item selection process.

As reflected in Table XI through XV there appeared overall to be moderately high correlations on most of the items within the 


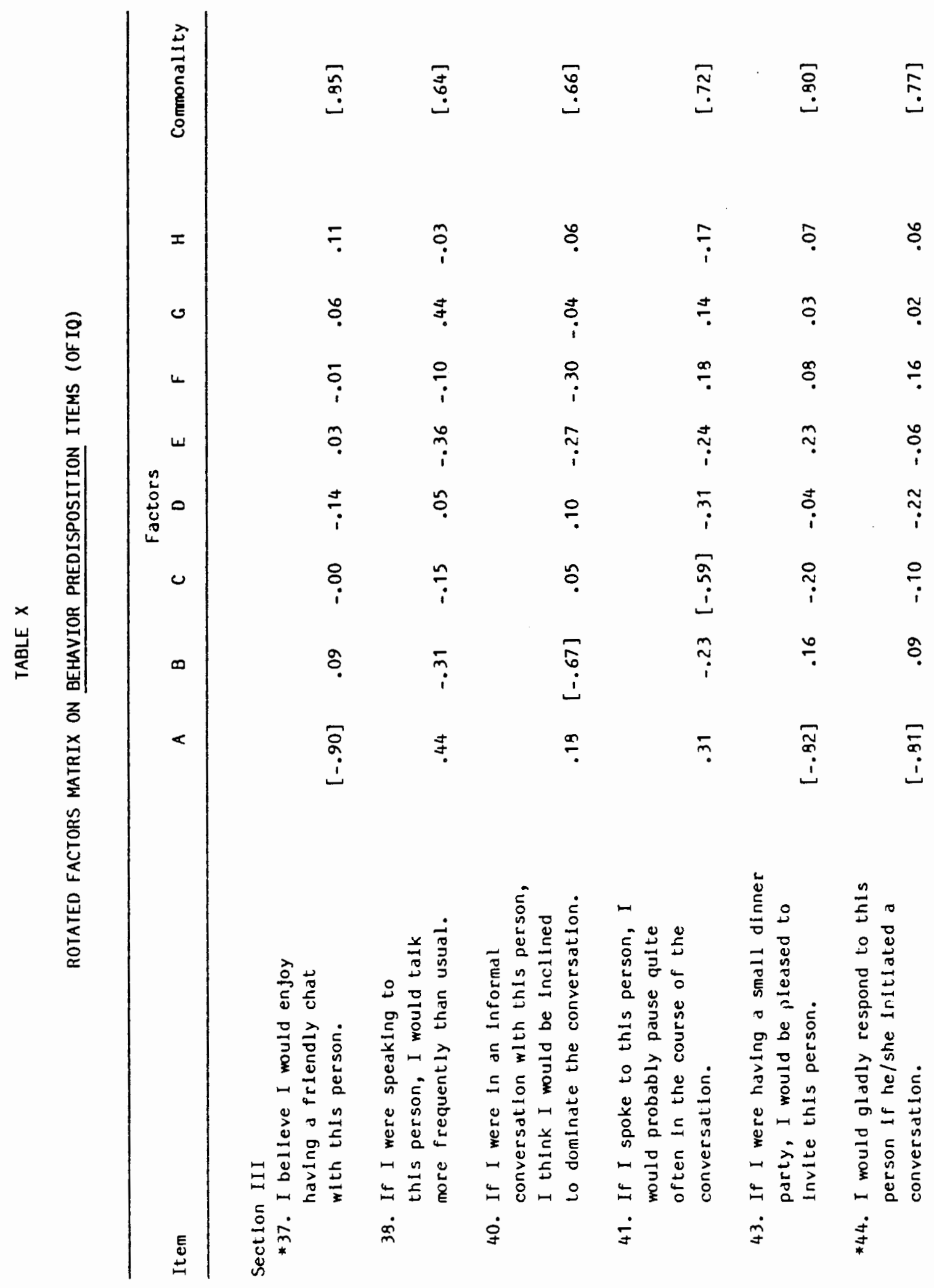




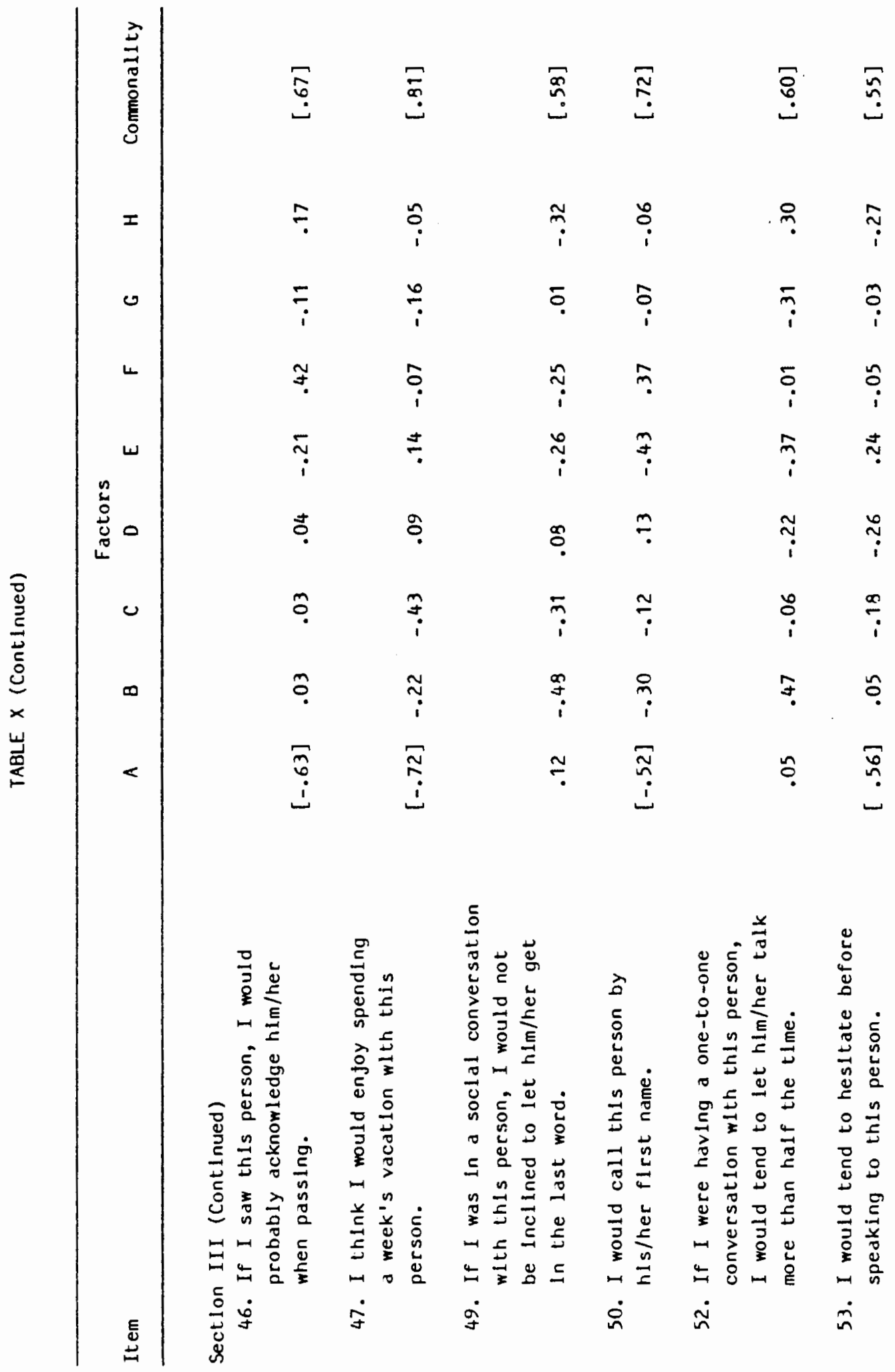




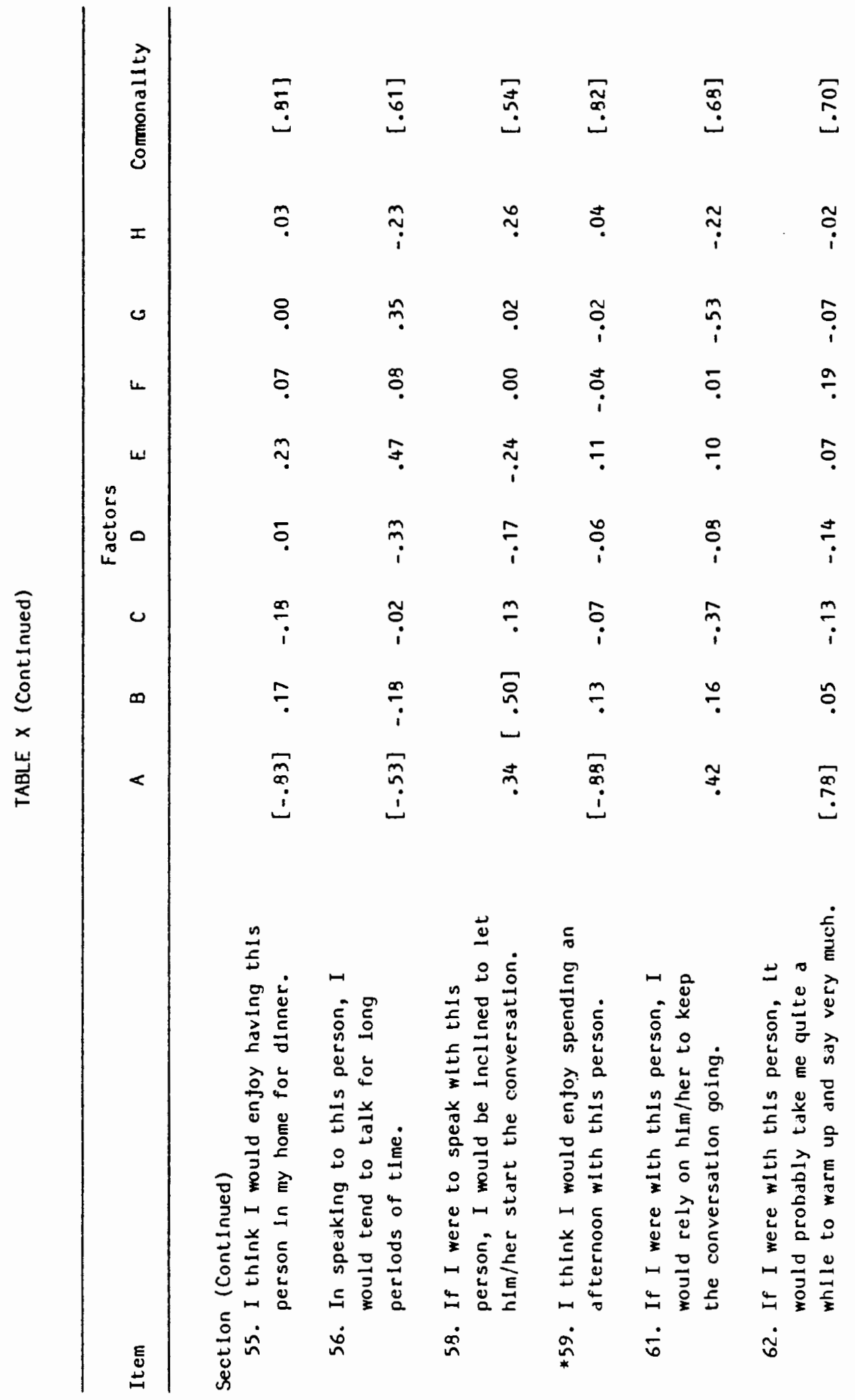




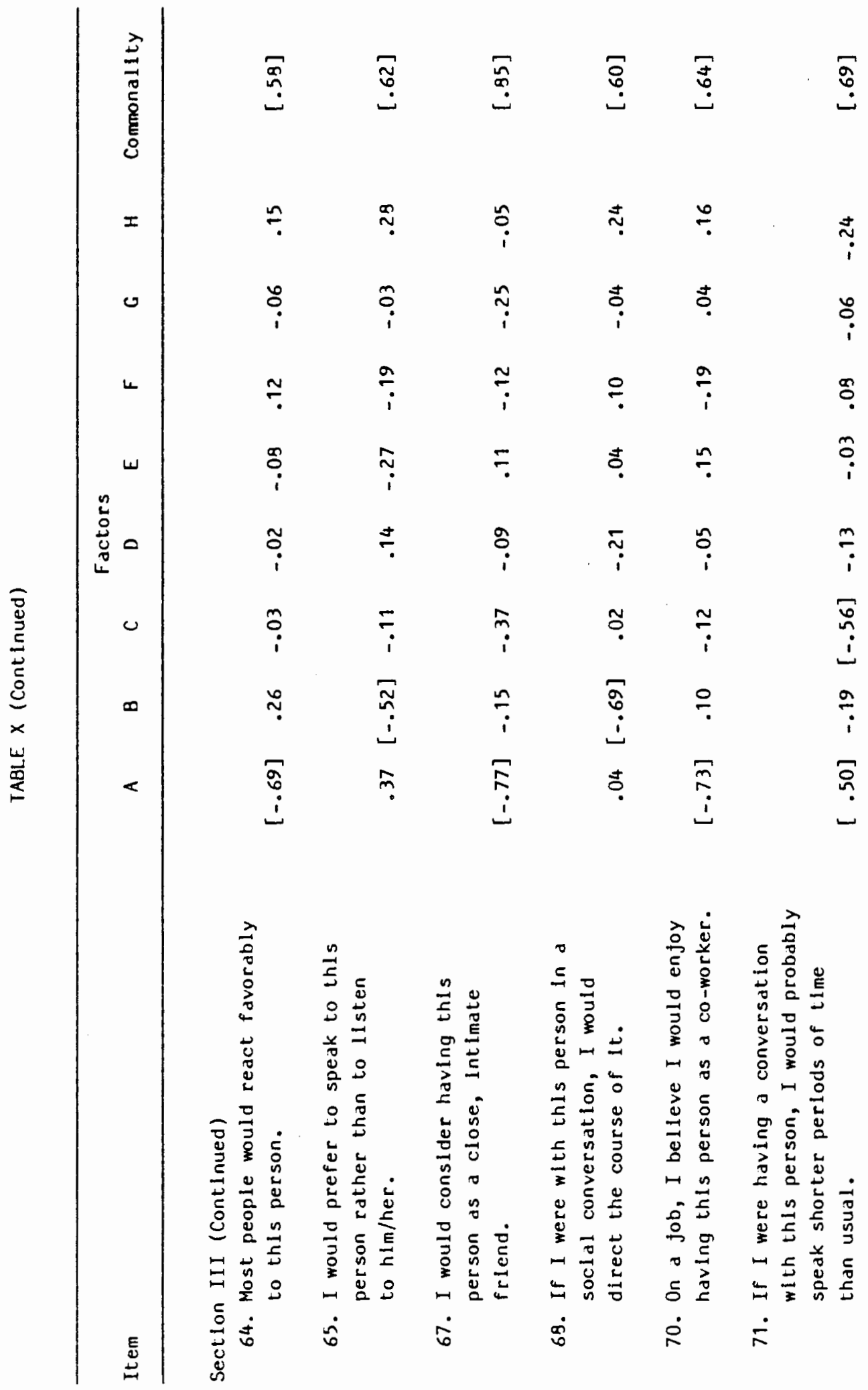




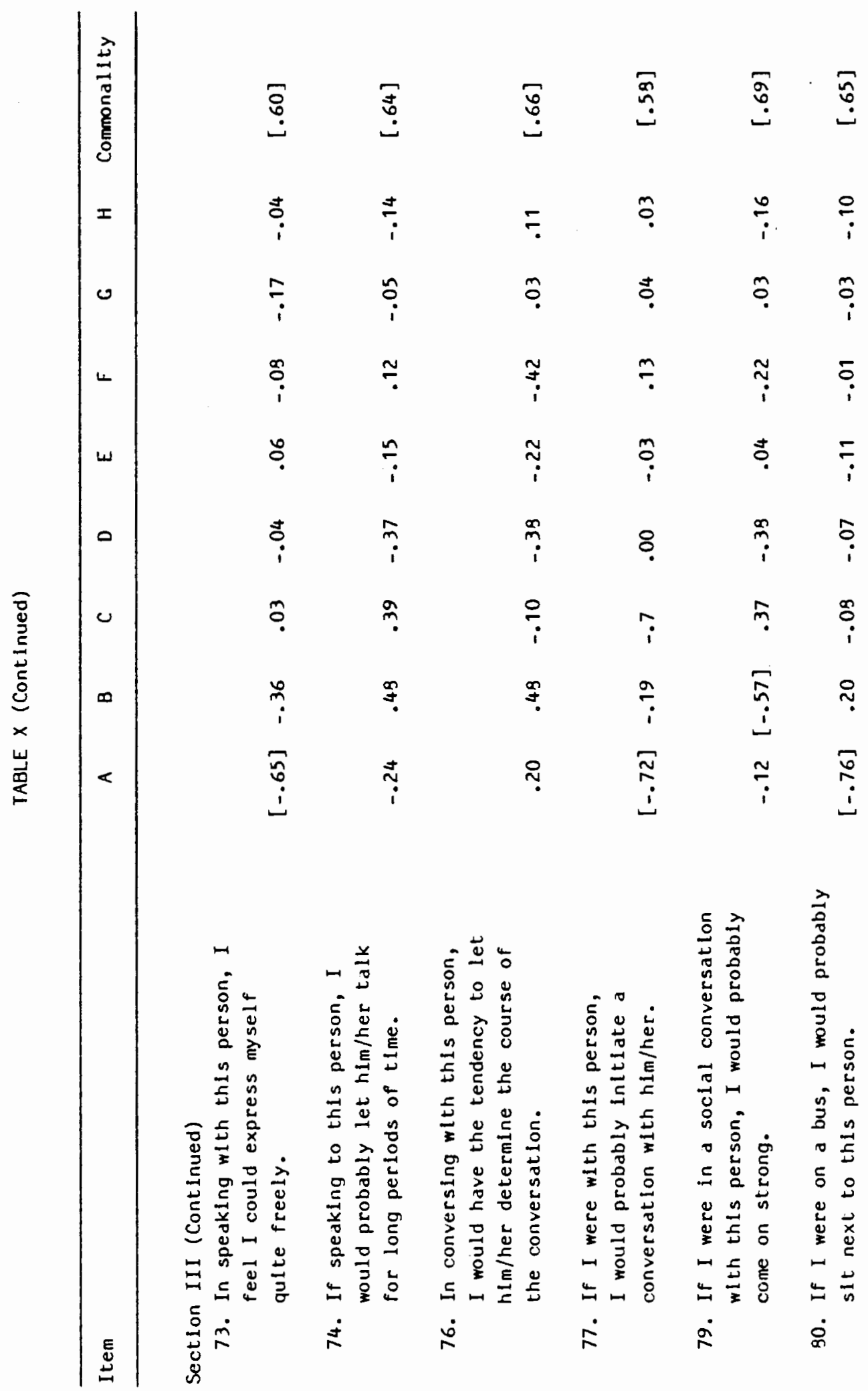




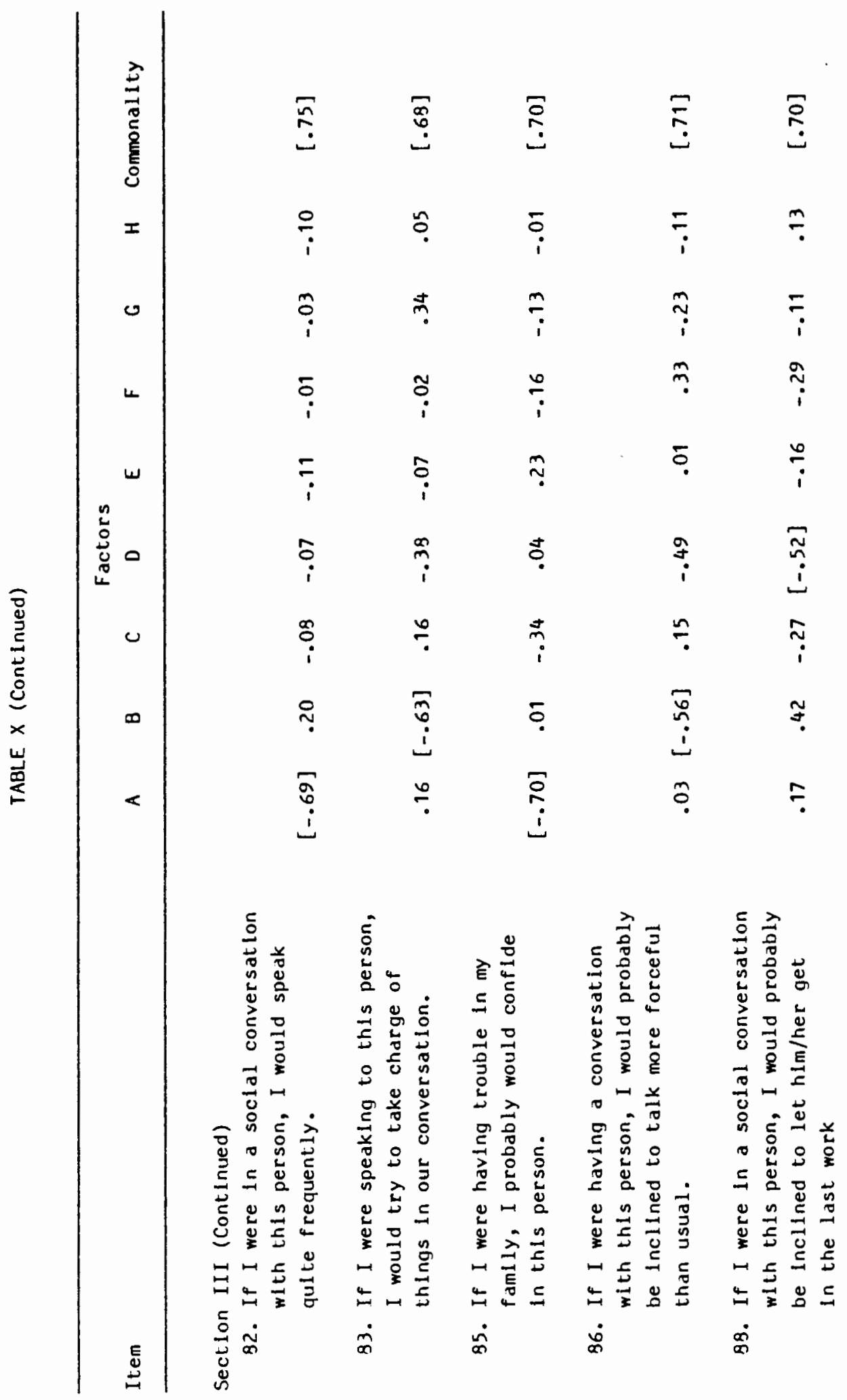




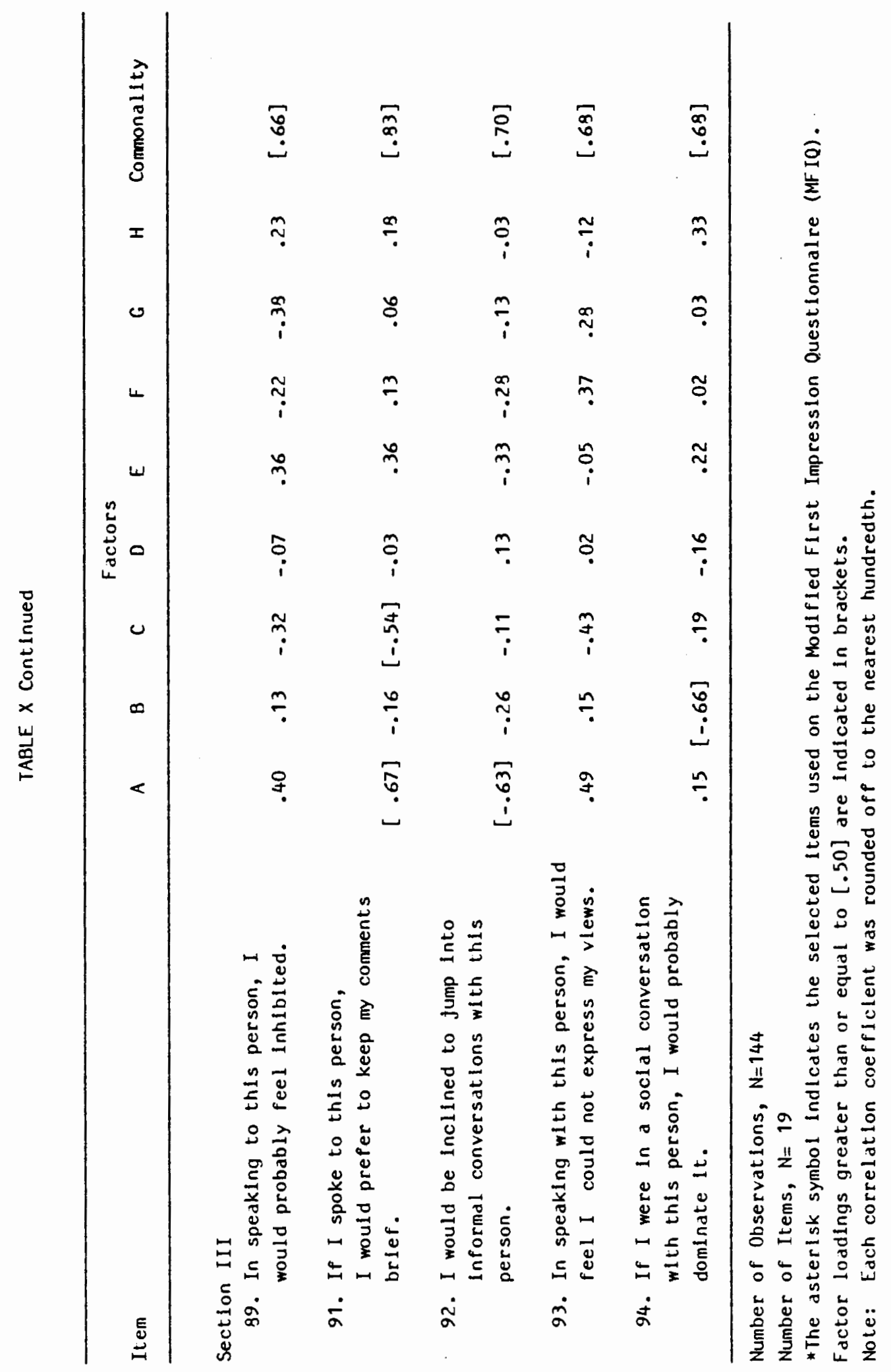


five components at the $p>.05$ level of significance. Each

computation on the Table reflects the degree of association between a given item on the test and retest. This is discussed in greater detail below.

\section{Physical Attributes}

Summarized in Table XI, Physical Attribute items appear to have moderate to high Pearson $r$ correlation coefficients between the test and retest. It is interesting to note the high correlation of Item 20, Hair $(r=.61)$ and the lower correlation coefficient Item 9, Hairstyle $(r=.56)$. The discrepancy between these two items, Hair and Hairstyle was somewhat incongruent. Nevertheless, a possible account for the incongruence of the questionnaire judges' responses to Hair and Hairstyle may be found in semantics. Item 1, Physical Appearance had a moderately high correlation coefficient of $r=.60$ while item (22) Very Physically Attractive to Very Physically Unattractive had a high degree of association, $r=.72$. The only two items that had an extremely low correlation coefficient associated with the test and retest were Item 7 , Weight $(r=.40)$ and Item 13 , Posture $(r=.40)$, possibly the result of what was shown in the photograph (i.e., full-face, neck-up photographs). The selected items for the Modified First Impression Questionnaire were 1, 5, 6, 9, 12, 15, 16, 18, 21 and 22 (a total of ten items), ranging from moderately high to high correlation coefficients of $r=.56$ to $r=.72$; see Table XI. 
TABLE XI

TEST-RETEST PEARSON $r$ CORRELATION COEFFICIENTS ON

PHYSICAL ATTRIBUTES ITEMS (OFIQ)

\begin{tabular}{ll} 
Item & $\underline{r}$ \\
\hline & \\
Section I & \\
*1. Physical Appearance & .60 \\
2. Nose & .58 \\
3. Face Shape & .62 \\
4. Eyebrows & .69 \\
*5. Facial Grooming & .69 \\
*6. Smile & .58 \\
7. Weight & .40 \\
8. Teeth & .64 \\
*9. Hairstyle & .56 \\
10. Personal Hygiene & .48 \\
11. Chin & .56 \\
*12. Eyes & .58 \\
13. Posture & .36 \\
14. Mouth & .60 \\
*15. Facial Expression & .67 \\
*16. Skin & .67 \\
17. Ears & .80 \\
18. Heaith & .58 \\
19. Neck & .62 \\
20. Hair & .61 \\
21. Well Groomed & .60 \\
22. Very Physically Attractive- & \\
Very Physically Unattractive & .72
\end{tabular}

$\mathrm{p}<.05$ level of significance

Number of observations, $N=72$

*The asterisk symbol indicates the selected items on the Modified

First Impression Questionnaire (MFIQ).

Note: Each Pearson $r$ Correlation Coefficient was rounded off to the nearest hundredth. 


\section{Cognitive}

Instrumental qualities. Table XII presents all the

Instrumental items Pearson $r$ correlational coefficient degree of association between the test and retest. Item 23, "Independent-Dependent," had the lowest correlation coefficient of $r=.34$ while item 35, "Alert--Non-alert," at $r=.68$, had the highest degree of association between the test and retest. The items selected for the Modified questionnaire were $25,27,31,33$ and 35 . These items' Pearson $r$ correlation coefficient ranged from $r=.47$ to $r=.68$. Items with a high coefficient were sometimes not selected for the Modified questionnaire since other statistical computations besides Pearson $r$ were considered in the decision-making process. It is interesting to note that the overall Instrumental Qualities correlation coefficients were not as high as Physical Attribute items.

Interpersonal qualities. As indicated on Table XIII, Interpersonal Component Pearson $r$ Correlation Coefficients reveal that al 1 the items had fairly high correlations between the test and retest. Item 100, "Friendly--Unfriendly" had the lowest Pearson $r$ coefficient, $r=.33$, while Item 24, "Pleasant--Unpleasant," ( $r=.56)$ was the highest correlation coefficient between the test and retest. It should be noted that both the Instrumental Quality and Interpersonal Quality component items have lower correlations than Physical Attribute component items. The selected items on the Interpersonal component were $24,28,32,34,36$ (a total of 5 items) to be used on the Modified questionnaire (see Table XIII). 
TEST-RETEST PEARSON $r$ CORRELATION COEFF ICIENTS ON INSTRUMENTAL QUALITIES ITEMS (OFIQ)

Section II

23. Independent-Dependent

*25. Active-Passive

*27. Enthusiastic-Unenthusiastic

29. Fast-Slow

*31. Strong-Weak

*33. Amb i tious-hardworking/Unambitious-l azy

*35. Alert-Non-alert .34 .64 .65 .46 .53

Section IV

96. Intell igent-Un intell igent .57

98. Positive Self-Concept/Negative Self-Concept 102. Tidy-Untidy

$p<.05$ levels of significance.

Number of observations, $\mathrm{N}=72$

*The asterisk symbol indicates the selected items used on the Modified First Impression Questionnaire (MFIQ). Note: Each Pearson $r$ correlation coefficient was rounded off to the nearest hundredth. 
TABLE XIII

TEST-RETEST PEARSON $r$ CORRELATION COEFFICIENTS ON INTERPERSONAL QUALITIES ITEMS (OFIQ)

Section II

*24. Pleasant-Unpleasant

.56

26. Open minded-Closed minded

.51

*28. Considerate-Inconsiderate

.51

30. Loving-Unloving

.43

*32. Cooperative-Uncooperative

*34. Understanding-Not understanding

*36. Flexible-Inflexible

Section IV

95. Kind-Cruel

97. Modest-Immodest

99. Sensitive-Insensitive

100. Sincere-Insincere

.45

101. Friendly-Unfriendly

104. Sophisticated-Unsophisticated

. .42

105. Cold-Warm

$p<.05$ levels of significance. Number of observations, $\mathrm{N}=72$

*The asterisk symbol indicates the selected items used on the Modified First Impression Questionnaire (MFIQ). Note: Each Pearson $r$ correlation coefficient was rounded off to the nearest hundredth. 
The correlational coefficients of these items ranged from $r=.51$ to $r=.56$ at $p>.05$ levels of significance.

\section{$\underline{\text { Affect }}$}

As reflected in Table XIV, the Affect items' Pearson $r$ Correlation Coefficients were all moderately high between the test and retest. It can be seen on Table XIV that the overall Pearson $r$ correlation coefficients were moderately high to high in comparison to the Instrumental and Interpersonal Quality component. Item 63 had. the lowest correlation $r=.30$, while Item 69 had the highest coefficient of $r=.69$. The asterisk symbol on the table indicates the selected items used on the Modified questionnaire were items 42,45 , 54, 60 and 90 (a total of five items) with correlation coefficents ranging from $r=.57$ to $r=.69$.

\section{Behavior}

Behavior predisposition. Overall, Pearson $r$ correlation coefficients for Behavior Predisposition items as seen on Table XV, were not as high as Physical Attributes or Affect component coefficients. As previously indicated, the Instrumental Qualities, Interpersonal Qualities and Behavior Predisposition items had a lower degree of association between the test and retest. Neverthless, the items of these three components still ranged from Moderate to high in their coefficients. The five Behavior Predisposition items selected for the Modified First Impression Questionnaire were 37, 44, 59, 67 and 77 . In these selected items, Pearson $r$ correlation ranged from $r=.52$ to $r=.74$ as shown on Table XV. 
TABLE XIV

TEST-RETEST PEARSON $r$ CORRELATION COEFFICIENTS ON AFFECT ITEMS (OFIQ)

\begin{tabular}{|c|c|}
\hline Item & $r$ \\
\hline $\begin{array}{l}\text { Section II } \\
\text { 39. I think I would enjoy knowing this person. } \\
\text { *42 I am attracted to this person. } \\
\text { *45. I think I would admire this person. } \\
\text { 48. I think I could feel close to this person. } \\
\text { 51. I feel favorable towards knowing this person. } \\
\text { *54. This person appeals to me. } \\
\text { 57. I think I would feel amiable towards this person. } \\
\text { *60. I am interested in knowing this person. } \\
\text { 63. I feel I can identify with this person. } \\
\text { 66. I would feel warmly towards this person. } \\
\text { 69. I wish I could meet this person. } \\
\text { 72. I think this person is similar to me. } \\
\text { 75. This persons is repul sive to me. } \\
\text { 78. I feel I could become friends with this person. } \\
\text { 81. I would feel fond of this person. } \\
\text { 84. I would love to meet this person. } \\
\text { 87. I feel I would dislike knowing this person. } \\
\text { *90. I feel I would like this person. }\end{array}$ & $\begin{array}{l}.68 \\
.69 \\
.64 \\
.46 \\
.71 \\
.65 \\
.67 \\
.66 \\
.30 \\
.38 \\
.52 \\
.44 \\
.39 \\
.56 \\
.65 \\
.45 \\
.55 \\
.57\end{array}$ \\
\hline $\begin{array}{l}\text { Section IV } \\
\text { 103. Likeable-Unlikeable }\end{array}$ & .55 \\
\hline
\end{tabular}

Number of observations, $\mathrm{N}=72$

*The asterisk symbol indicates the selected items used on the Modified First Impression Questionnaire (MFIQ).

Note: Each Pearson $r$ correlation coefficient was rounded off to the nearest hundredth. 
TABLE XV

TEST-RETEST PEARSON $r$ CORRELATION COEFFICIENTS ON BEHAVIOR PREDISPOSITION ITEMS (OF IQ)

Item

$r$

Section III

*37. I believe I would enjoy having a

friendly chat with this person.

38. If I were speaking to this person,

I would talk more frequently than usual.

40. If I were in an informal conversation

with this person, I think I would be

inclined to dominate the conversation.

41. If I spoke to this person, I would probably pause quite often in the course of the conversation.

43. If I were having a small dinner party, I would be pleased to invite this person.

*44. I would gladiy respond to this person if he/she initiated a conversation.

46. If I saw this person, I would probably acknowledge him/her when passing.

47. I think I would enjoy spending a week's vacation with this person.

49. If I was in a social conversation with

this person, I would not be inclined

to let $\mathrm{him} / \mathrm{her}$ get in the last word.

50. I would call this person by his/her first name. $\quad .58$

52. If I were having a one-to-one conversation with this person, I would tend to let him/her talk more than half the time. 
TABLE XV (Continued)

Item

Section III (Continued)

53. I would tend to hesitate before speaking to this person.

55. I think I would enjoy having this person

in my home for dinner.

56. In speaking to this person, I would tend to talk for long periods of time.

58. If I were to speak with this person, I would be inclined to let him/her start the conversation.

*59. I think I would enjoy spending an afternoon with this person.

61. If I were with this person, I would rely on him/her to keep the conversation going.

62. If I were with this person, it would probably take me quite a while to warm up and say very much.

64. Most people would react favorably to this person.

65. I would prefer to speak to this person rather than to listen to him/her.

*67. I would consider having this person as a close, intimate friend.

68. If I were with this person in a social conversation, I would direct the course of it.

70. On a job, I believe I would enjoy having

this person as a co-worker.

71. If I were having a conversation with this person, I would probably speak shorter periods of time than usual.

73. In speaking with this person, I feel I

could express myself quite freely.

74. If speaking to this person, I would probably let him/her talk for long periods of time. 
TABLE XV (Continued)

Item

Section I I (Continued)

76. In conversing with this person, I would have the tendency to let him/her determine the course of the conversation.

*77. If I were with this person, I would probably initiate a conversation with him/her.

79. If I were in a social conversation with this person, I would probably come on strong.

80. If I were on a bus, I would probably sit next to this person.

82. If I were in a social conversation with this person, I would speak quite frequently.

83. If I were speaking to this person, I would try to take charge of things in our conversation.

85. If I were having trouble in my family, I probably would confide in this person.

86. If I were having a conversation with this person, I would probably be inclined to talk more forceful than usual.

88. If I were in a social conversation with this person, I would probably be inclined to let $\mathrm{nim} /$ her get in the last word.

89. In speaking to this person, I would probably feel inhibited.

91. If I spoke to this person, I would prefer to keep my comments brief.

92. I would be inclined to jump into informal conversations with this person.

93. In speaking with this person, I would feel I could not express my views. 
TABLE XV (Continued)

Item

$r$

Section II (Continued)

94. If I were in a social conversation with this person, I would probably dominate it.

Number of observations, $N=72$

*The astrisk symbol indicates the selected items used on the Modified First Impression Questionnaire (MFIQ).

Note: Each Pearson $r$ correlation coefficient was rounded off to the nearest hundredth. 


\section{RELIABILITY}

Reliability was used to assess how consistent the responses to each item on the 105 item questionnaire were between the test and the retest. The reliability of each item refers to how accurate the "true score" was in the population sampled in this study. So, if there was no error in measuring a given item, the reliability coefficient would be "one." According to Williams (1974) reliability was defined as the "external and internal consistency of measurement." Would the results be the same if the item on the questionnaire were tested and retested again under the same conditions? In other words, reliability is the "consistency" between the test and retest of each item on the OFIQ.

Tables XVI through XX, Discrimination Indices on each of the five components defining physical attractiveness multidimensional$1 y$, show the corrected item-total correlation and the alpha. The Model-split specification was used to determine how stable the responses were to the test and retest over time (Nie et al., 1970). Items were selected based upon a high corrected item-total correlation reliability and then compared to the correlational matrix as to how high that item was in comparison to the others. Alpha is the most widely used reliability coefficient. The asterisk symbols beside the item number on each of the tables represent the selected items used on the MFIQ. The specific process of selection based on reliability is described below. 
Physical attributes. Table XVI reflects the Discrimination Indices on Physical Attribute items. Nineteen of the 22 items had a corrected item-total correlation greater than or equal to .50. Three of the items had extremely low correlations: Item 8, teeth (.24); Item 14, posture (.36); and Item 17, ears (.33). Perhaps the reason why these items had such low correlations was that it was difficult for the judges to see the teeth, posture or ears of the people in the photograph.

All of the 22 items had extremely high alpha correlations ranging from .94 to .95 .

\section{Cognitive}

Instrumental qualities. An inspection of the Table XVII, Discrimination Indices on Instrumental Quality Items shows the high correlations on the corrected item-total and alpha correlations. The corrected item-total correlations ranged from .53 to .82; moderatley high to high coefficients.

All the items had high alpha coefficients indicating potential items for the MFIQ.

Interpersonal qualities. Table XVIII summarizes the corrected item-total correlation and alpha coefficients on the Interpersonal component. Thirteen of the 14 items had high correlations ranging from .58 to .87 . However, there was one item with an extremely low correlation of .31: "Sophisticated versus Unsophisticated." Again, all the alphas' correlation coefficients were extremely high. 
TEST-RETEST DISCRIMINATION INDICES ON

PHYSICAL ATTRIBUTES ITEMS (OFIQ)

\begin{tabular}{|c|c|c|c|}
\hline \multicolumn{2}{|l|}{ Item } & $\begin{array}{l}\text { Corrected Item- } \\
\text { Total Correlation }\end{array}$ & Alpha \\
\hline $\begin{array}{r}\text { Sectio } \\
\star 1 . \\
2 . \\
3 . \\
4 . \\
\star 5 . \\
\star 6 . \\
7 . \\
8 . \\
\star 9 . \\
10 . \\
11 . \\
\star 12 . \\
13 . \\
14 . \\
\star 15 . \\
\star 16 . \\
17 . \\
\star 18 . \\
19 . \\
20 . \\
\star 21 . \\
\star 22 .\end{array}$ & $\begin{array}{l}\text { I } \\
\text { Physical Appearance } \\
\text { Nose } \\
\text { Face Shape } \\
\text { Eyebrows } \\
\text { Facial Grooming } \\
\text { Smile } \\
\text { Weight } \\
\text { Teeth } \\
\text { Hairstyle } \\
\text { Personal hygiene } \\
\text { Chin } \\
\text { Eyes } \\
\text { Posture } \\
\text { Mouth } \\
\text { Facial expression } \\
\text { Skin } \\
\text { Ears } \\
\text { Health } \\
\text { Neck } \\
\text { Hair } \\
\text { Well groomed } \\
\text { Very Physically Attractive- } \\
\text { Very Physicall Unattractive }\end{array}$ & $\begin{array}{l}.82 \\
.71 \\
.72 \\
.71 \\
.76 \\
.72 \\
.50 \\
.24 \\
.60 \\
.70 \\
.63 \\
.73 \\
.36 \\
.76 \\
.80 \\
.79 \\
.33 \\
.75 \\
.73 \\
.67 \\
.70\end{array}$ & $\begin{array}{l}.94 \\
.94 \\
.94 \\
.94 \\
.94 \\
.94 \\
.95 \\
.95 \\
.94 \\
.94 \\
.94 \\
.94 \\
.95 \\
.94 \\
.94 \\
.94 \\
.95 \\
.94 \\
.94 \\
.94 \\
.94\end{array}$ \\
\hline
\end{tabular}

Number of observations, $\mathrm{N}=144$

Number of items, $\mathrm{N}=22$ Physical Attributes

*The asterisk symbol indicates the selected items used on the Modified First Impression Questionnaire (MFIQ).

Note: Each correlation coefficient was rounded off to the nearest hundredth. 


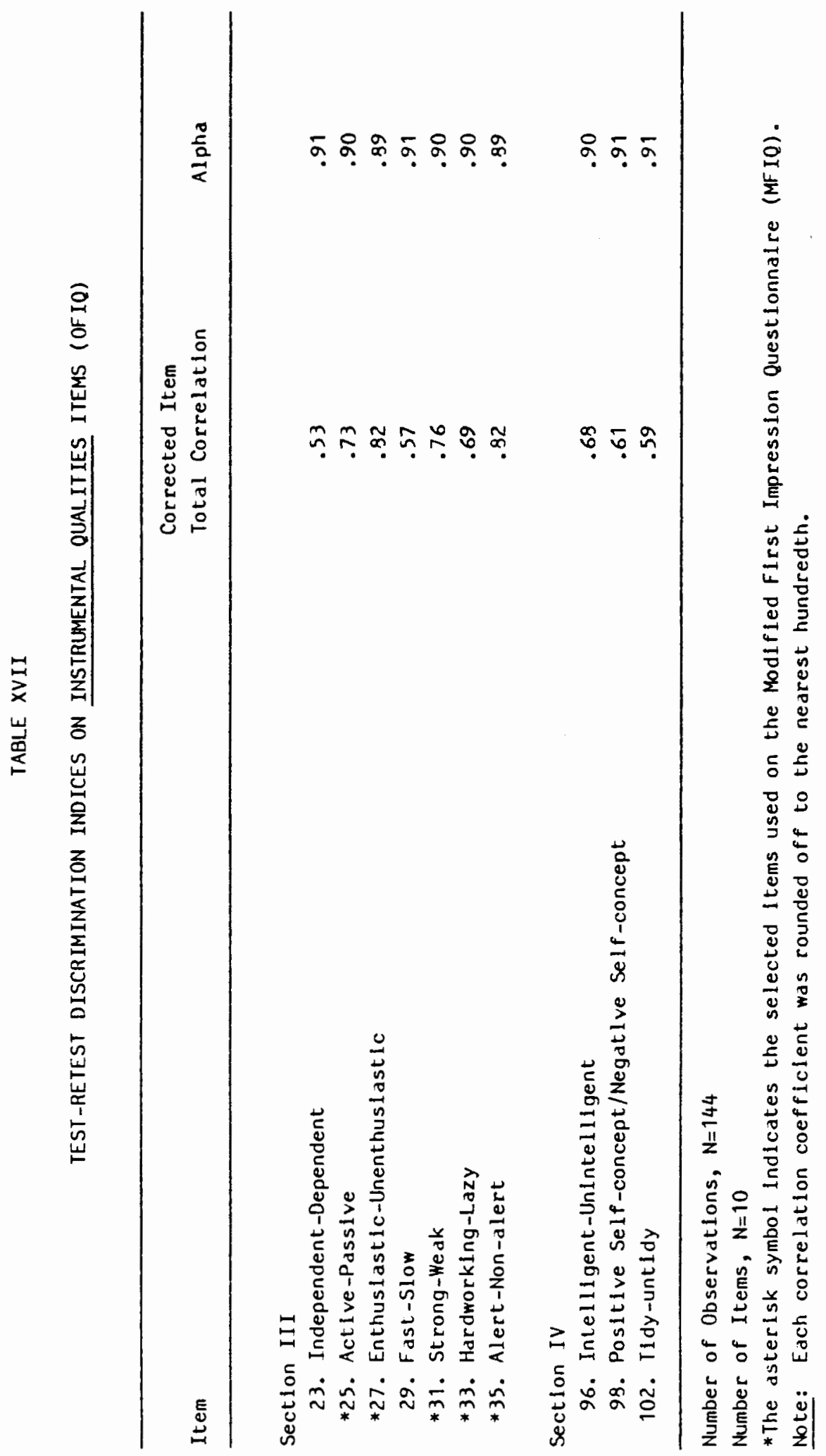




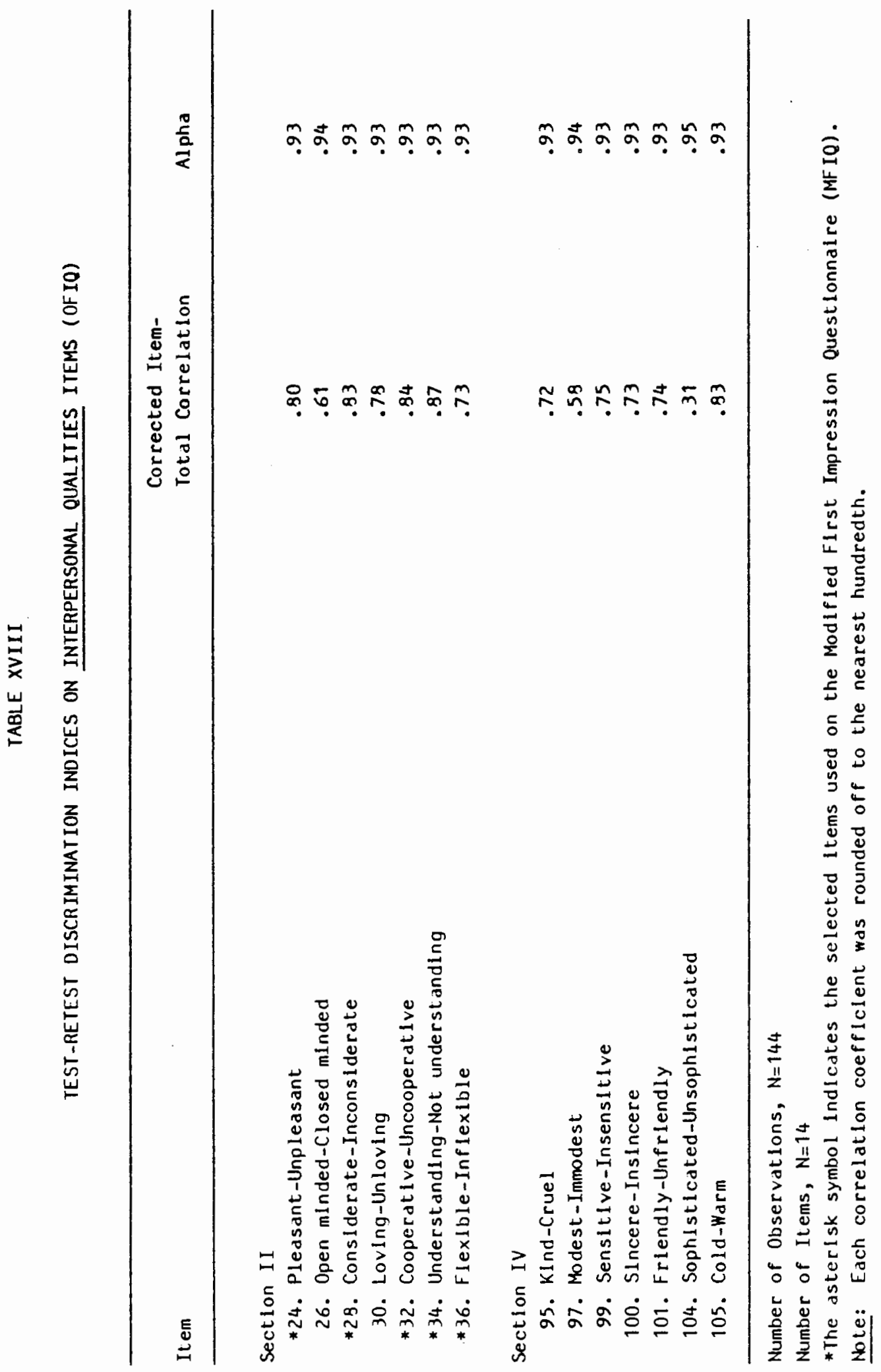




\section{Affect}

As indicated on Table XIX, Discrimination Indices on Affect items ranged from moderately high to high corrected item-total correlations of .53 to .90 .

The alpha correlations for all the items were extremely high except for Item 87, "I feel I would dislike knowing this person," with .60 . Generally speaking, all the items were considered as potential items to be selected for the MFIQ.

Behavior

Behavior Predisposition. Table $X X$ shows the Discrimination Indices on Behavior Predispositon items with corrected item-total correlation and alpha coefficients. Fourteen of the 40 Behavior Predisposition items had high corrected item-total correlation items had high factor loadings greater than or equal to .53 .

All the Behavior Predisposition items had high alpha correlation coefficients. The items with both the high corrected item-total correlation and alpha were considered to be valid items for use on the questionnaire. 


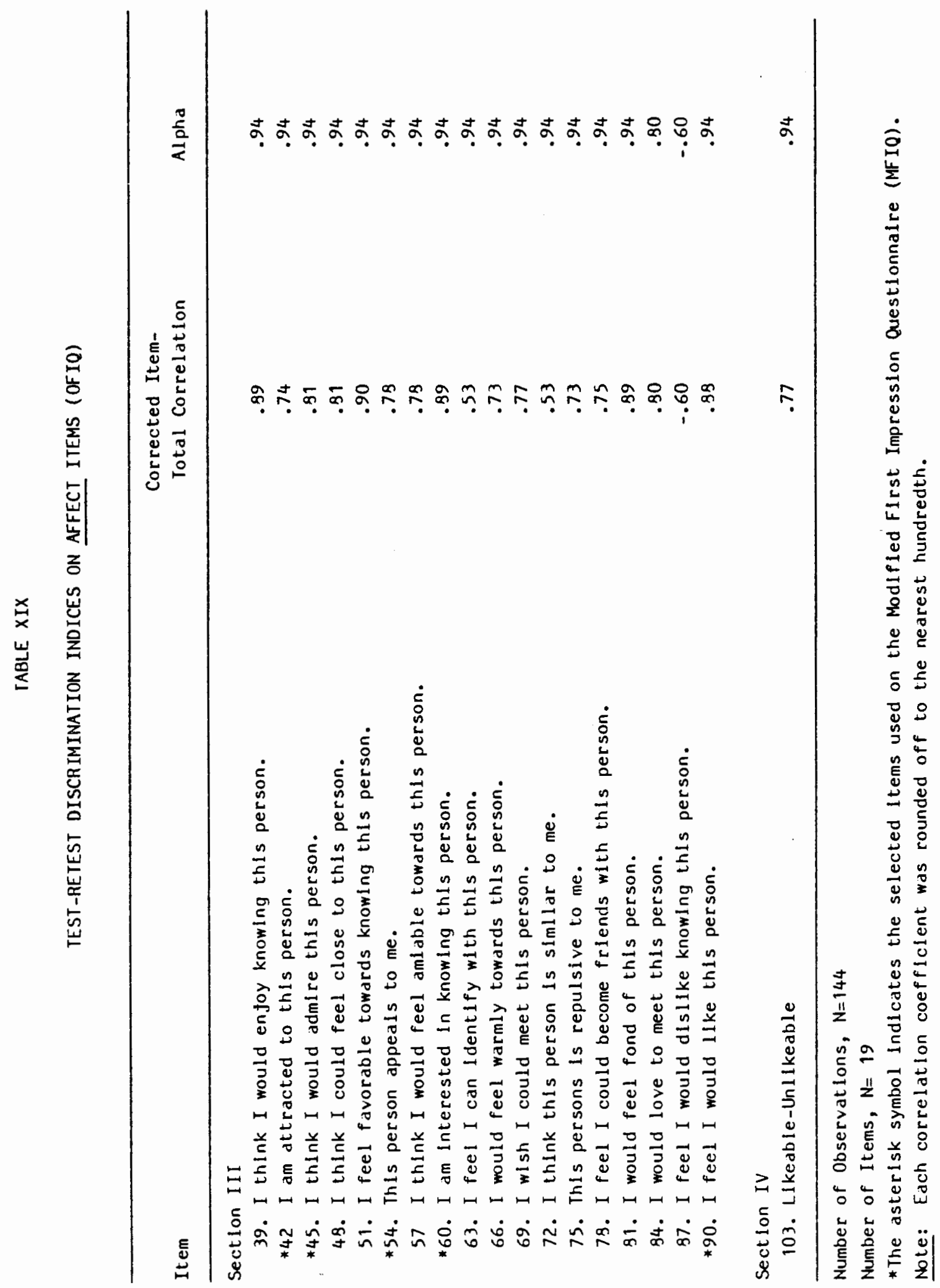




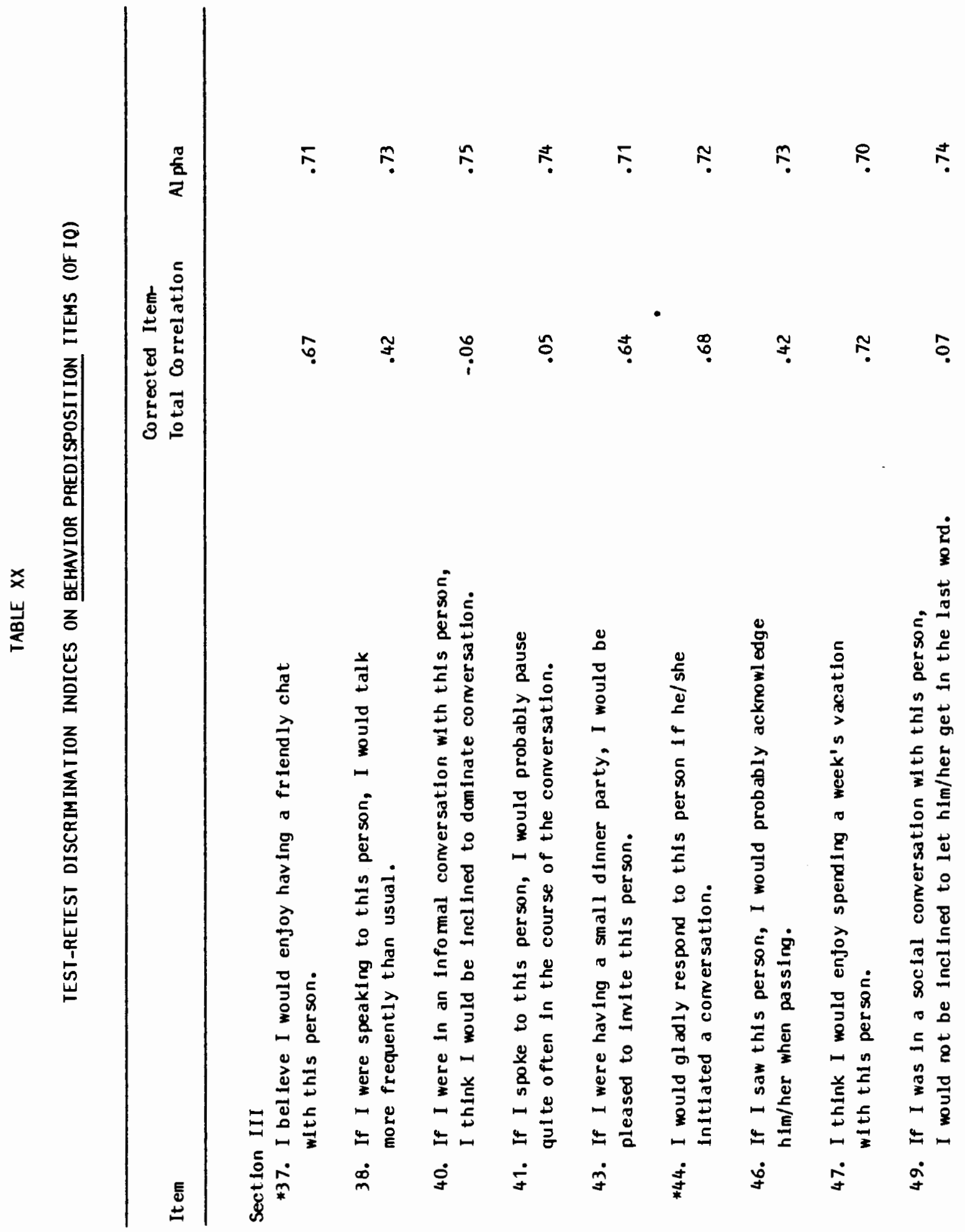




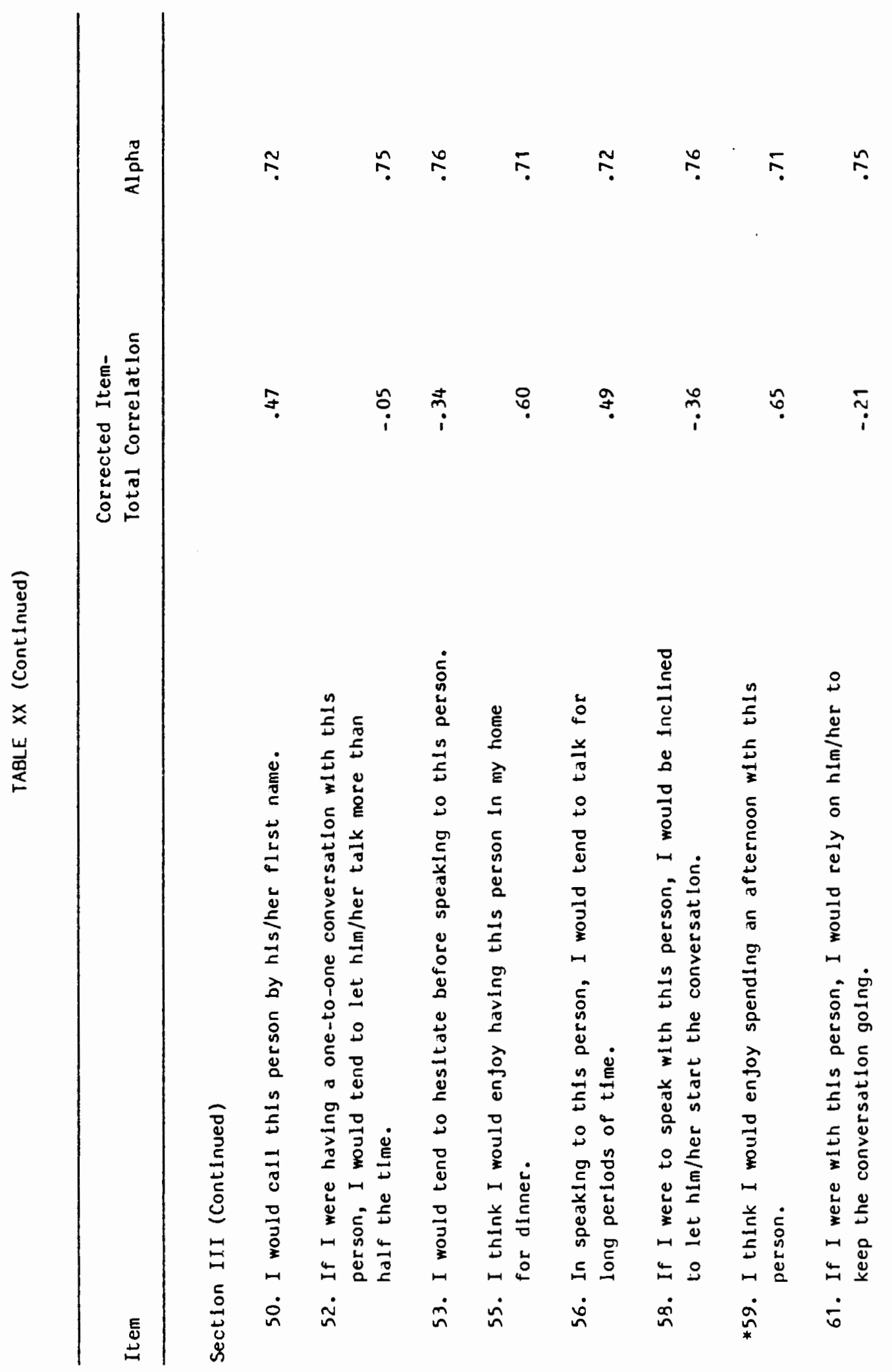




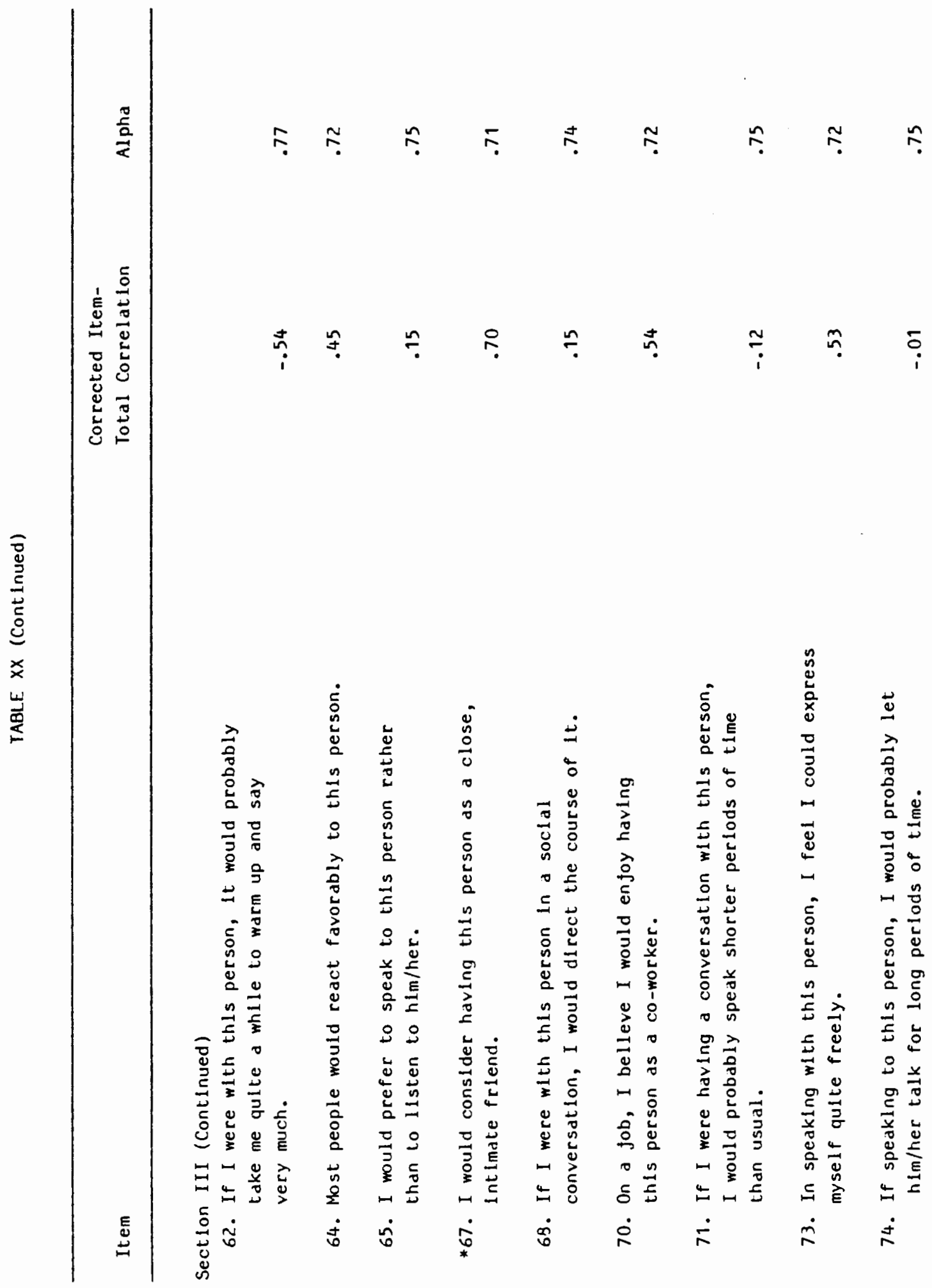




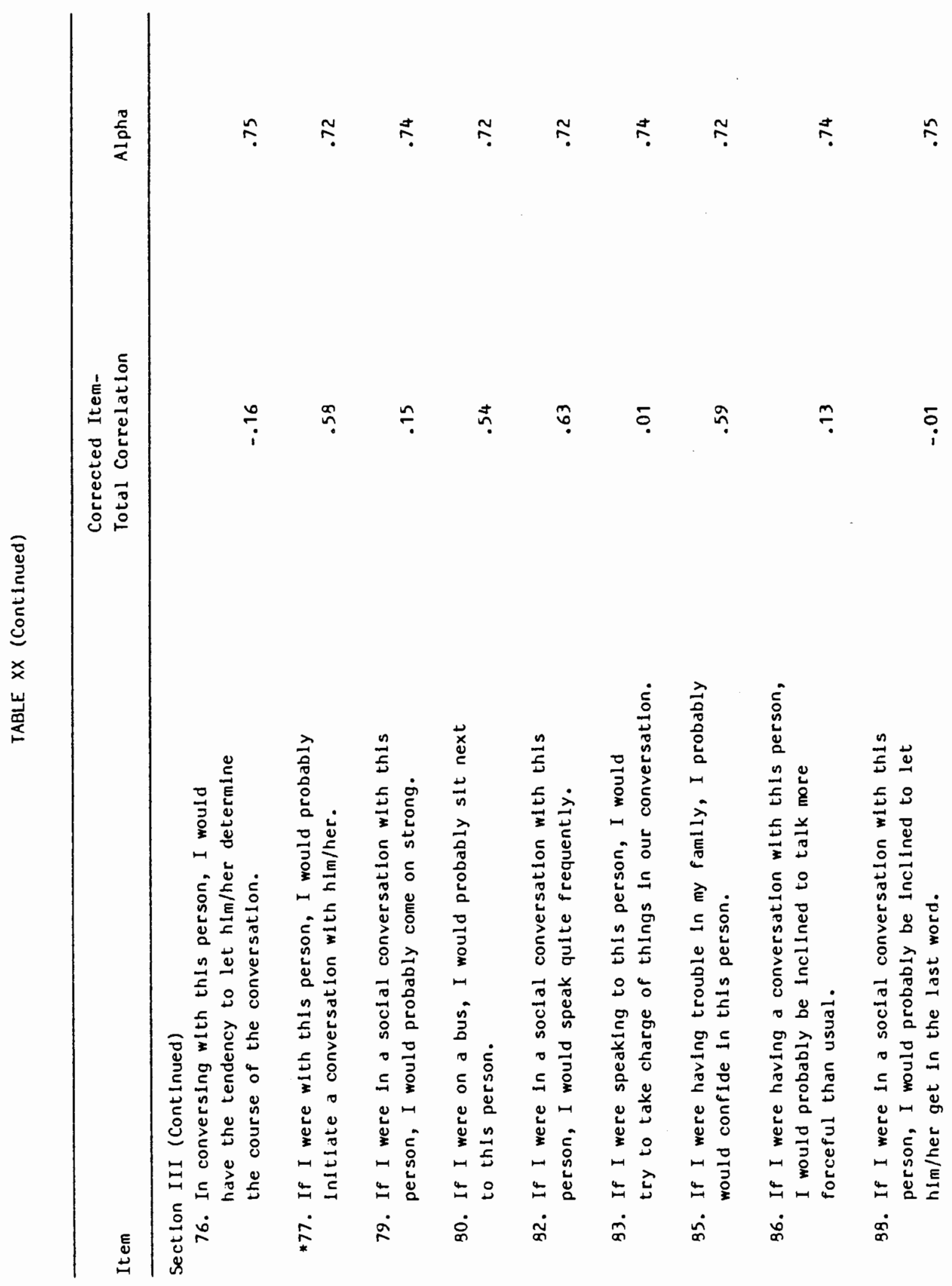




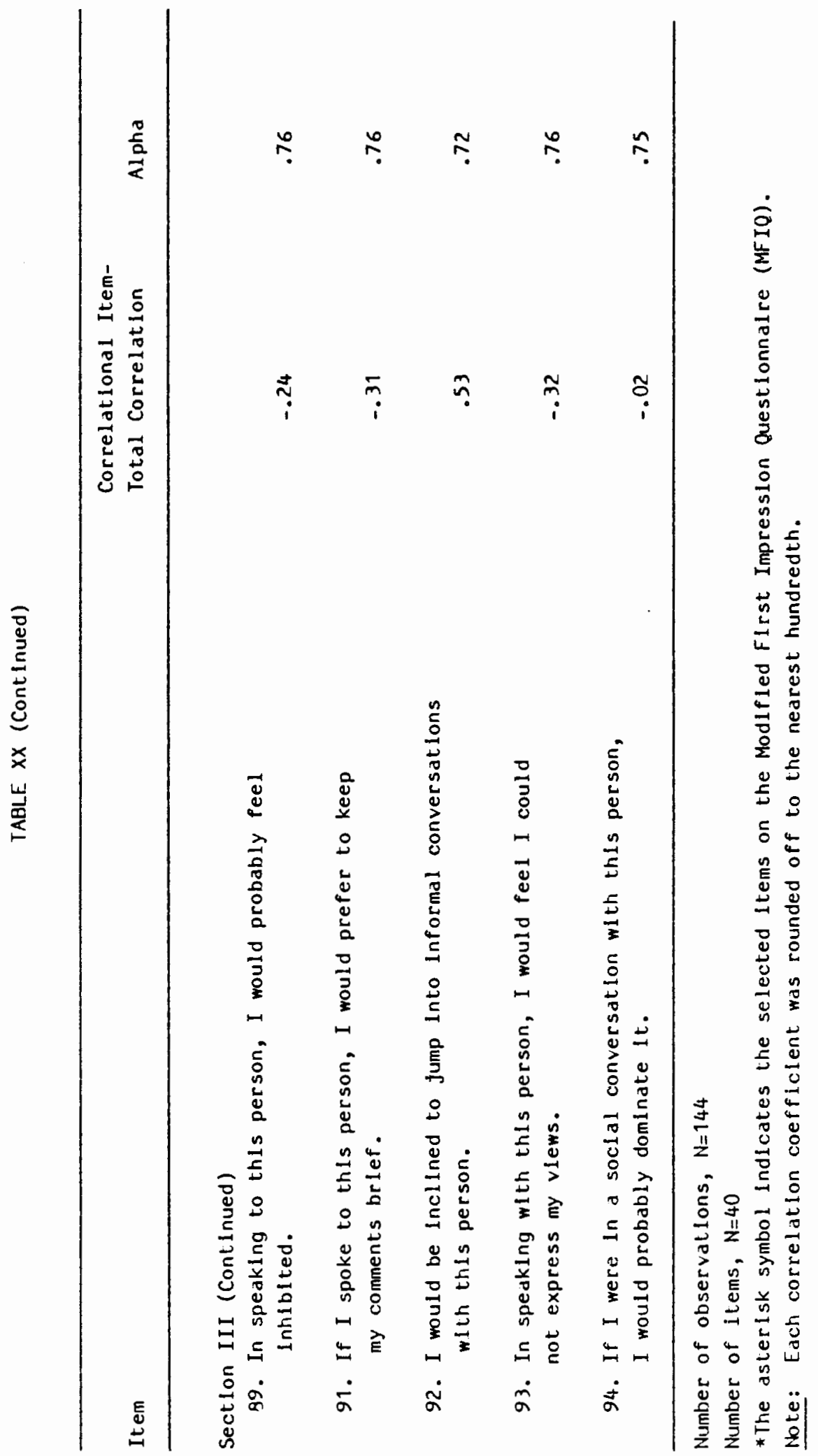


FINAL SELECTION OF ITEMS FOR MFIQ

Introduction

After the 105 items on the Original questionnaire had been pared down to 30 items on the Modified version, the revised questionnaire was again statistically analyzed. The investigator used descriptive statistics, reliability and factor analyses on all of the items on the Modified version of the questionnaire to determine the correlations among the "selected items." The selected items from all five components defining physical attractiveness were constructed into a questionnaire called the Modified First Impression Questionnaire (MFIQ). The MFIQ (see Appendix D) was used in Section IV of this study. Figure 8 , below, reflects the selected items within each component used on the MFIQ.

\begin{tabular}{lll}
\hline $\begin{array}{c}\text { Questionnaire } \\
\text { Section }\end{array}$ & Item & Total No.Component \\
& of Items
\end{tabular}

(1) Physical Attributes

1

$1,2,3,4,5,6$, $7,8,9,10$

(2) Instrumental

(3) Interpersonal

(4) Affect

(5) Behavior

Predisposition
2

2

3

3
$11,13,15,17,19$

$12,14,16,18,20$

$21,23,25,27,29$

$22,24,26,28,30$
10 5 5 5

Number of items, $N=30$

Figure 8. Items on the Modified Physical Attractiveness Interpersonal First Impression Questionnaire. 


\section{DESCRIPTIVE STATISTICS}

Table XXI summarizes the Descriptive Statistics on the Modified First Impression Questionnaire Items, including all five components. As indicated on the table, means and standard deviations were statistically computed under one of the five components categorizing (1) Physical Attributes; (2) Instrumental Qualities; (3) Interpersonal Qualities; (4) Affect and (5) Behavior Predisposition. Generally, all the items had means between the range 3 to 4 except Items 14, 26, 29 and 30. The overall means reflect the judgements' normal distribution. The mean values were toward the middle of the category range, rather than the extremes. It appears from Table XXI that Physical Attributes, Instrumental Qualities and Interpersonal Qualities means consistently range 3.60 to 5.05 whereas on the other two components, Affect and Behavior Predisposition were generally lower, ranging from 2.00 to 4.88 . The standard deviation on all five components were fairly large allowing for less consensus of opinion on a given item. However, Item 30, "I think I would enjoy spending an afternoon with this person," (Behavior Predisposition component) had an extremely small standard deviation of $S D=.05$.

In summary, the selected items based upon these descriptive statistics were carefully chosen.

Reliability of MFIQ

Table XXII, Discrimination Indices on the five components 
TABLE XXI

\section{DESCRIPTIVE STATISTICS ON MODIFIED FIRST IMPRESSION QUESTIONNAIRE (MFIQ) ITEMS}

\section{Physical Attributes}

Section I

1. Physical Appearance

3.65

1.28

2. Hair

3.67

1.53

3. Facial Expression

4.02

1.49

4. Health

4.15

1.34

5. Eyes

6. Well Groomed

4.09

1.38

4.65

1.41

7. Skin

4.21

1.50

8. Smile

4.11

1.58

9. Facial Grooming

10. Very Physically Attractive-

4.47

1.38 Very Physically Unattractive

3.60

1.34

Instrumental Qualities

Section II

11. Active-Passive

4.33

1.43

13. Enthusiastic-Unenthusiastic

4.73

1.36

15. Strong-Weak

4.37

1.35

17. Ambitious, hardworkingUnambitious-lazy

4.88

1.27

19. Alert-Nonalert

4.82

1.29

Interpersonal Qualities

Section II

12. Considerate-Inconsiderate

4.98

1.20

14. Pleasant-Unpleasant

5.05

1.27

16. Flexible-Inflexible

4.40

1.27

18. Understanding-Not understanding

4.85

1.23

20. Cooperative-Uncooperative

4.93

1.19 
TABLE XXI (Continued)

Component

Item

Mean Standard Deviation

\section{Affect}

Section III

21. I feel I would like this person.

4.19

1.43

23. This person appeals to me.

3.47

1.50

25. I think I would admire this

3.31

1.46

27. I am interested in knowing this person.

3.02

1.47

29. I am attracted to this person.

2.84

1.53

\section{Behavior Predisposition}

Section III

22. I believe I would enjoy having

a friendly chat with this person.
4.17
1.54

24. If I were with this person, I would probably initiate a conversation with him/her.

26. I would consider having this

$3.41 \quad 1.63$
person as a close, initimate friend.

2.89

28. I would gladly respond to this person if he/she initiated a conversation.

$4.88 \quad 1.43$

30. I think I would enjoy spending an afternoon with this person.

2 .05

Number of observations, $\mathrm{N}=1,296$

Note: The mean and standard deviation was rounded off to the nearest hundredth. 
DISCRIMINATION INDICES ON THE MODIFIED INTERPERSONAL FIRST IMPRESSION QUESTIONNAIRE (MFIQ) ITEMS

\begin{tabular}{ll}
\hline Component & Corrected Item \\
Item & Total Correlation Alpha \\
\hline
\end{tabular}

Physical Attributes

Section I

1. Physical Appearance

.79

.92

2. Hair

3. Facial Expression

.72

.93

4. Health

.77

.92

5. Eyes

.75

.92

6. Well Groomed

.68

.93

7. Skin

.69

.93

8. Smile

.68

.93

9. Facial Grooming

10. Very Physically Attractive-

.74

.92

.70

.93

Very Physically Unattractive

Instrumental Qualities

Section II

11. Active-Passive

13. Enthusiastic-Unenthusiastic

.69

.84

.74

.82

15. Strong-Weak

.60

.86

17. Ambitious, hardworkingUnambitious-lazy

.68

.84

19. Alert-Nonalert

.73

.83

Interpersonal Qualities

Section II

12. Considerate-Inconsiderate $\quad .79$

.88

13. Pleasant-Unpleasant

.80

.87

15. Flexible-Inflexible

.62

.91

18. Understanding-Not understanding

20. Cooperative-Uncooperative

.81

.87

.77

.89

\section{Affect}

Section III

21. I feel I would like this person.

23. This person appeals to me.

25. I think I would admire this person.

27. I am interested in knowing this person.

29. I am attracted to this person. 
TABLE XXII (Continued)

Component

Item
Corrected Item

Total Correlation

Alpha

Behavior Predisposition

Section III

22. I believe I would enjoy having a friendly chat with this person.

.82

24. If I were with this person, I would probably initiate a conversation with $\mathrm{him} / \mathrm{her}$.

.70 .88

26. I would consider having this person as a close, intimate friend.

28. I would gladiy respond to this person if he/she initiated a conversation

30. I think I would enjoy spending an afternoon with this person.

Number of observations, $N=1,296$

Note: Each correlation coefficient was rounded off to the nearest hundredth. 
defining physical attractiveness, provides a summary of the reliabilities of the Modified First Impression Questionnaire. The corrected item-total correlational coefficients for all the items were high, ranging from .60 to .88 . Additionally, the alpha coefficients for all the items among the five components were extremely high.

\section{Factor Analysis of MFIQ}

As reflected in Table XXIII, Rotated Factor Matrix on MFIQ Items had high factor loadings and communality correlations. Instrumental Quality component, Item 15, and Interpersonal Quality component, Item 16, had low communalities correlation of .41 and .43. Other than that, all the items among the five components were high.

Summary

Based upon the statistical analysis, the Modified questionnaire had a total of 30 items. Figure 8 (p. 101) shows the number of items, sections' identification numbers, and total number of items within each of the five components defining physical attractiveness on the MFIQ.

MFIQ was divided up into three sections containing (1) Physical Attributes, (2) a combination of both Instrumental and Interpersonal Qualities and (3) a combination of Affect and Behavior Predisposition.

Format of items. The question format on the MFIQ remained the same as the OFIQ. 
TABLE XXIII

ROTATED FACTOR MATRIX ON MODIFIED FIRST IMPRESSION

QUESTIONNAIRE (MFIQ) ITEMS

Component

Item
Factor

A

Commonal ity

Physical Attributes

Section I

1. Physical Appearance

2. Hair

3. Facial Expression

4. Health

5. Eyes

6. Well Groomed

7. Skin

8. Smile

9. Facial Grooming

10. Very Physically AttractiveVery Physically Unattractive

$[-.82]$

$[-.76]$

$[-.80]$

$[-.78]$

$[-.70]$

$[-.71]$

$[-.71]$

$[-.77]$

[-.72]

$[-.84]$
[.68]

$[.57]$

$[.65]$

$[.61]$

$[.50]$

[.51]

$[.50]$

[.59]

$[.51]$

[.71]

Instrumental Qualities

Section II

11. Active-Passive

13. Enthusiastic-Unenthusiastic

15. Strong Weak

17. Ambitious, hardwork-unambitious lazy

19. Alert-Nonalert

[-.74]

$[-.82]$

$[-.64]$

$[-.75]$

$[-.81]$
$[.55]$

$[.68]$

.41

[.56]

$[.66]$

\section{Interpersonal Qualities}

Section II

12. Considerate-Inconsiderate

14. Pleasant-Unpleasant

16. Flexible-Inflexible

18. Understanding-Not understanding

20. Cooperative-Uncooperative

$[-.84]$
$[-.85]$
$[-.65]$
$[-.87]$
$[-.83]$

$[.72]$

$[.73]$

.43

$[.75]$

$[.68]$

\section{Affect}

\section{Section III}

21. I feel I would like this person.

23. This person appeals to me.

25. I think I would admire this person.

$\begin{array}{ll}{[-.83]} & {[.68]} \\ {[-.92]} & {[.85]} \\ {[-.82]} & {[.67]}\end{array}$


TABLE XXIII (Continued)

Component

Item
Factor

A

Commonality

Affect (continued)

27. I am interested in knowing this person.

29. I am attracted to this person.

Behavior Predisposition

Section III

22. I believe I would enjoy having a friendly chat with this person.

$[-.88] \quad[.77]$

24. If I were with this person, I would probably initiate a conversation with him/her.

$[-.75]$

26. I would consider having this person as a close, intimate friend.

$[-.83]$

28. I would gladly respond to this person if he/she initiated a conversation.

30. I think I would enjoy spending an afternoon with this person.

$[-.87]$

$[.76]$

Number of observations, $\mathrm{N}=1,296$

Factor loadings greater than or equal to .50 are indicated in brackets.

*The asterisk symbol indicates the selected items used on the Modified First Impression Questionnaire (MFIQ).

Note: Each correlation coefficient was rounded off to the nearest hundredth. 
Presentation of items on the questionnaire. The order in which the items were presented on the questionnaire will be discussed briefly to indicate any variations that differed from the OFIQ.

In all sections, the similar items were separated so that the subjects were forced to cognitively evaluate the photographs without automatically responding. Physical Attributes was a section in itself describing personal physical characteristics. All the Instrumental and Interpersonal Qualities components were reordered into the followng pattern: Instrumental/Interpersonal/Instrumental. Affect and Behavior Predisposition items were also randomized in Section II to alternate.

The newly created 30 item questionnaire known as the MFIQ was used in the next section to address the asserted hypotheses of this study, as discussed in Section IV.

\section{SECTION IV}

Section IV describes how subjects rated the stimulus photographs by completing the questionnaire to test the hypotheses of the study. The photographs are the same pictures used in Sections II and III. The questionnaire used in this part of the study was the MFIQ developed and constructed in Section III. In order to test the hypotheses, a matrix with physical attractiveness intensity levels was created to develop six photo orders that included both degrees of physical attractiveness and age groups. The re-mainder of this section will discuss the subjects, procedures and data preparation 
used in analyzing the responses of the subjects as they evaluated the physical attractiveness of the stimulus photographs.

Matrix with Physical Attractiveness Intensity Levels for Stimulus Photographs

Section III described how a matrix was created with physical attractiveness intensity levels for the stimulus photographs in order to control for an "order effect." Intensity levels of high, medium, and low had already been established for the photographs in Section II. In Section III, numbers were assigned to these photographs indicating the degree of physical attractiveness by: (1) low, (2) medium or (3) high. Additionally, a matrix was designed to include all three levels of physical attractiveness intensity levels in which the questionnaire judges were presented with one age group of the stimulus photographs. The pictures used in Section IV were the same ones with the attached numerical value describing each photographs' physical attractiveness intensity levels.

Based upon the physical attractiveness intensity levels used in Section III, the photographs in all three photo orders were reassigned arbitrary numbers of 1 through 18 . This included all three age groups from Section III combined to create the photo orders for Section IV. The numbers were merely a convenience for the investigator and no one else saw them. The numbers were reassigned to the photographs for the purposes of creating six photo 
orders although the physical attractiveness intensity level of the photograph remained the same as determined in Section II and was assigned a numerical value of high, medium, or low in Section III.

\section{Subjects}

The subjects consisted of 72 participants between the ages of 19-24 years old (Young). There were 36 male and 36 female subjects. The mean age was $20.47(S D=1.64)$ for the total of 72 subjects; 36 male subjects' mean age was $20.33(S D=1.65)$ and 36 female subjects' mean age was $20.60(S D=1.65)$.

\section{Presentation of Stimulus Photographs to Subjects}

The presentation of photographs to the subjects was standardized into six photo orders so that each subject received only one of them. Figure 9 illustrated the photo orders used in this part of the study.

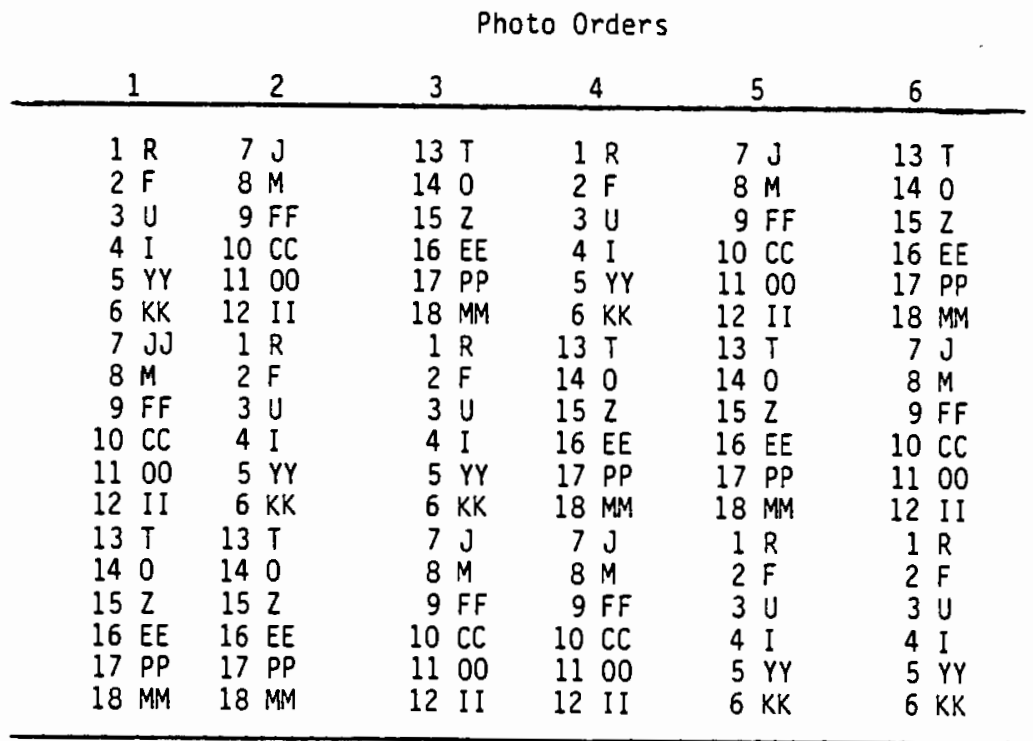

Number of Stimuli, $N=18$

Figure 15. Matrix used in creating six photo orders. 
Appendix $H$ contains the stimulus photographs of all three age groups. Figures 12 through 17 (see Appendix I) show the six photo orders with their identifiable physical attractiveness intensity levels beside the letter representing each picture.

Procedures Subjects used in Evaluating Stimulus Photographs' Physical Attractiveness.

The investigator met with each subject at an assigned room on campus, Portland State University, at scheduled times and days. The investigator introduced herself and provided the subject with the necessary forms.

Forms. The subject was given a Consent Form, Note on Anonymity and SES (See Appendix $B$ and D) to complete. Upon completion of these forms, the subject was given a total of 18 questionnaires with which to evaluate 18 stimulus photographs in assigned photo order. The stimulus photographs were contained in a notebook that the subject did not have any visual contact with until the preliminary forms had been completed.

MFIQ. Section III discussed how the MFIQ was created and described the format of the items. Subjects were given examples of the instructions for each of the three types of items that differed in format, as seen in Appendix $D$.

At the conclusion of the interview, the subject was informed that upon his/her request, he/she would receive a copy of the abstract report of the research findings. The subjects were personally thanked for participating in the study, and a thank-you letter was mailed to them within a week of their participation. 
DATA PREPARATION

The computed data analysis and results are discussed in Chapter IV, which addresses each hypothesis. The following paragraphs review only the preparation of data for analysis.

The data preparation to use a computer program called the Statistical Package for Social Scientists (referred hereafter as SPSS) by Nie et al. (1970). The statistics used in this study for Section III were: frequency runs, descriptive statistics, reliabilities factor analysis, Pearson $r$ correlations and for Section IV were: one-way t-tests and analysis of variance. This part of the planning was a separate matter even though later it was included in the process of data preparation.

The coding for both the OFIQ and the MFIQ involved first laying out a "code sheet." A code sheet is used primarily by the investigator to encode information from questionnaires into a machinereadable medium so that analysis can be accomplished. This new medium will enable the investigator to key punch computer cards or input responses from the questionnaires into a computer terminal. It would be cumbersome to write out all the responses on the questionnaire for each participant. So, a coding scheme was devised by listing the questionnaire $i$ tems and assigning numeric values to each response. Appendix $H$ contains the coding sheets for both the OFIQ and the MFIQ. The code sheets include a column number and the question number beside each item.

Numerical values were assigned to the ratings by coding each scale as follows: 
Attractive

or

Strongly Agree

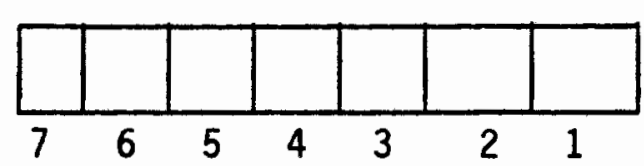

Unattractive

or

Strongly Disagree

The assignment of numbers was such that the positive characteristics on both questionnaires were assigned larger numbers and negative characteristics smaller numbers. In this manner, the investigator could "eyeball" the numbers and immediately know the larger numbers on the computer printout would indicate that the subjects evaluated the stimulus photographs more positively in physical attractiveness. This method avoids cumbersome negative numbers and possible misinterpretation.

If an item was left blank, missed or not applicable it was called "missing data" and coded on the computer as " 0 ". The missing data was extremely low due to the investigator being present at each interview. Therefore, it did not significantly effect the interpretation of the results.

A total score for the subjects rating the stimuli was computed for each of the five components consisting of Physical Attributes, Instrumental Qualities, Interpersonal Qualities, Affect and Behavior Predisposition. This was done by adding each item number chosen by the subject within the component for each of the pictures. This procedure is explained in greater detail in Chapter IV.

Variables on the questionnaire could be coded into one of two types for input into the SPSS system: numeric or alphanumeric. 
Numeric variables were selected by the investigator since alphanumeric has a limited number of statistical procedures, extra processing time is required and there is some awkwardness in referencing the manipulated information on the questionnaire. Some basic knowledge of the SPSS program is important when one decides how to layout data.

Computer cards were selected to input responses into the computer. Cards were chosen by the investigator instead of using the computer terminal in order to have a copy of the data and because it is less expensive to run the program. If there is a power shortage while using a computer terminal, all inputed data will be lost unless there is a back-up disk. The investigator duplicated a second copy of the data key punched on computer cards.

Fortran sheets were used to code the variables prior to keypunching the computer cards. A Fortran sheet is basically a spread sheet with 80 columns (spaces) across the page, in rows. Once the data has been coded and punched, it may be input into the SPSS directly from the punched cards.

Some of the cards define and describe the data as it is entered into the system; others allow the user to control the operation of the statistical program and yet others enable the user to recode and transform the data in the file.

The next chapter describes the statistical analysis used to answer the hypotheses in this study. 


\section{CHAPTER IV}

\section{RESULTS}

This chapter reports the findings of the research questions and hypotheses of this study. The tabular presentation of all analytical results is provided in Tables XXIV through XXXII. The three hypotheses addressed in this thesis are directional. Therefore, all hypothesis testing comparisons following initial F-tests are of an "a priori" nature and would be computed regardless of analysis of variance results.

A total score was computed for each of the five components, defining physical attractiveness multidmensionally by adding each item number chosen by the subject within that component for each of the stimulus photographs.

The statistical analyses used to examine the research questions and hypotheses were descriptive statistics of mean age group comparisons, one-way t-test and analysis of variance on each of the five components. The investigator wrote the programs to be used in the statistical analysis. The calculations were performed by a Honeywell level $66 / 40$ computer using the implementation of Statistical Package for Social Sciences (referred to as SPSS). Each hypothesis will be stated prior to reporting the results of its statistical analysis. 


\section{HYPOTHESIS I}

Hypothesis I asserts that the Young Female stimuli will be rated more positively in physical attractiveness than either the Middle-Aged or 01der stimulus photographs by subjects in the 19 to 24 year old age group, (Young).

In order to answer Hypothesis I in this study, some necessary statistical analyses had to be selected, computed and interpreted to determine the findings. This is discussed below.

\section{Descriptive Statistics}

Table XXIV and XXV present the mean comparisons of age groups, standard error and one-way t-test. The table indicates each component, showing the statistical computation to answer Hypothesis I. In order to see a comparison of the statistical computations based upon the subjects' evaluations of the stimulus photographs, young male and young female subjects' responses were examined independently. To address the first hypothesis, contrasted age groups of the stimulus photographs were isolated in order to differentiate the computations more easily, based upon the subjects' responses.

Young male subjects rating young, middle-age, old female stimulus photographs. Table XXIV shows how young male subjects rate Young/01d Female stimulus photographs. Statistical results indicate the following computations for each component: Physical Attributes $t(320)=1.044, p>.05$, (significant); Instrumental Qualities $t(321)=2.022, p=.044$; Interpersonal Qual ities $t(321)=2.294, p=.022$; 
TABLE XXIV •

YOUNG MALE SUBJECTS RATING YOUNG, MIDDLE-AGED, OLD FEMALE STIMULUS PHOTOGRAPHS

\begin{tabular}{|c|c|c|c|c|c|}
\hline$\frac{\text { Component }}{\text { Comparisons }}$ & Mean & $\begin{array}{c}\text { Standard } \\
\text { Error }\end{array}$ & DF & t Value & $\mathrm{p}$ \\
\hline \multicolumn{6}{|l|}{ Physical Attributes } \\
\hline $\begin{array}{l}\text { Young/ } \\
\text { old }\end{array}$ & $\begin{array}{l}43.6481 \\
40.3084\end{array}$ & 11.4609 & 320 & 1.044 & .297 \\
\hline $\begin{array}{l}\text { Middle/ } \\
\text { old }\end{array}$ & $\begin{array}{l}42.0926 \\
40.3084\end{array}$ & 10.1802 & 320 & 1.195 & .233 \\
\hline $\begin{array}{l}\text { Young/ } \\
\text { Middle }\end{array}$ & $\begin{array}{l}40.3084 \\
42.0926\end{array}$ & 11.1697 & 320 & 2.236 & .026 \\
\hline \multicolumn{6}{|c|}{ Instrumental Qualities } \\
\hline $\begin{array}{l}\text { Young/ } \\
\text { old }\end{array}$ & $\begin{array}{l}-24.3056 \\
23.3458\end{array}$ & 4.1094 & 321 & 2.022 & .044 \\
\hline $\begin{array}{l}\text { Middle/ } \\
01 \mathrm{~d}\end{array}$ & $\begin{array}{l}22.9633 \\
23.3458\end{array}$ & 5.6403 & 321 & .575 & .566 \\
\hline $\begin{array}{l}\text { Young/ } \\
\text { Middle }\end{array}$ & $\begin{array}{l}23.3458 \\
22.9633\end{array}$ & 4.7921 & 321 & 1.439 & .151 \\
\hline \multicolumn{6}{|c|}{ Interpersonal Qualities } \\
\hline $\begin{array}{l}\text { Young/ } \\
\text { old }\end{array}$ & $\begin{array}{l}26.3148 \\
26.5421\end{array}$ & 3.4333 & 321 & 2.294 & .022 \\
\hline $\begin{array}{l}\text { Middle/ } \\
\text { old }\end{array}$ & $\begin{array}{l}24.9908 \\
26.5421\end{array}$ & 4.7616 & 321 & 2.681 & .008 \\
\hline $\begin{array}{l}\text { Young/ } \\
\text { Middle }\end{array}$ & $\begin{array}{l}26.5421 \\
24.9908\end{array}$ & 4.4410 & 321 & .392 & .695 \\
\hline \multicolumn{6}{|l|}{ Affect } \\
\hline $\begin{array}{l}\text { Young/ } \\
\text { old }\end{array}$ & $\begin{array}{l}19.2222 \\
17.5514\end{array}$ & 6.4755 & 321 & 2.778 & .006 \\
\hline $\begin{array}{l}\text { Middle/ } \\
\text { old }\end{array}$ & $\begin{array}{l}16.8716 \\
17.5514\end{array}$ & 6.0140 & 321 & .801 & .423 \\
\hline $\begin{array}{l}\text { Young/ } \\
\text { Middle }\end{array}$ & $\begin{array}{l}17.5514 \\
16.8716\end{array}$ & 6.2029 & 321 & 1.965 & .050 \\
\hline \multicolumn{6}{|c|}{ Behavior Predisposition } \\
\hline $\begin{array}{l}\text { Young/ } \\
\text { old }\end{array}$ & $\begin{array}{l}20.8981 \\
18.6168\end{array}$ & 6.3569 & 321 & 3.767 & .000 \\
\hline $\begin{array}{l}\text { Middle/ } \\
\text { old }\end{array}$ & $\begin{array}{l}17.8807 \\
18.6168\end{array}$ & 5.7199 & 321 & .917 & .360 \\
\hline $\begin{array}{l}\text { Young/ } \\
\text { Middle }\end{array}$ & $\begin{array}{l}18.6168 \\
17.8807\end{array}$ & 5.5925 & 321 & 2.835 & .005 \\
\hline
\end{tabular}

Number of observations, $\mathrm{N}=72$

$p<.05$ NS

$p>.05 \mathrm{~S}$ 
Affect $t(321)=2.778, p=.006$, and Behavior Predisposition $t(321)=3.767, p=.0000$. The Middle/01d significant interaction effects had the following t-values: Physical Attributes $t(320)=1.195$, Instrumental Qualities $t(321)=.575$; Affect $6(321)=.801$; and Behavior Predisposition $t(321)=.917$. The last age group comparison of the stimulus photographs were young male subjects, rating Middle-Aged Female pictures with Physical Attributes $t(320)=2.236, p=.026$; Instrumental Qualities $t(321)=1.439, p>.05$, (significant); Interpersonal Qualities $t(321)=.392, p>.05$, (significant); Affect $t(321)=1.965, p>.050$ (significant); and Behavior Predisposition $t(321)=2.835, p>.005$, (significant).

Young Female subjects rating young, middle-aged, old female stimulus photographs. Table XXV summarizes the mean comparisons between age groups, standard error and $t$-value of each component. Young female subjects rating Female stimulus photographs by contrasting age group comparisons show the Young/01d age group computations with $t$-values of the following: Physical Attributes $t(321)=1.677$, $\mathrm{p}=.095$; Instrumental Qualities $\mathrm{t}(321)=1.401, \mathrm{p}>.05$ (significant); Interpersonal Qualities $t(321)=2.294, p=.022$; Affect $t(320)=.071$, p >.05 (significant); and Behavior Predisposition $t(321)=.927$, $p>.05$ (significant). As reflected on Table XXV, Middle-Aged/01 d age group comparisons of the Female photographs showed t-values on Physical Attributes $t(321)=.619, p>.05$ (significant); Instrumental Qualities $t(321)=1.038, p>.05$ (significant); Interpersonal Qualities $t(320)=2.566, p=.011$; Affect $t(320)=1.846, p>.05$ (significant); and Behavior Predisposition $t(321)=1.505, p>.05$ (significant). 
TABLE XXV

YOUNG FEMALE SUBJECTS RATING YOUNG, MIDDLE-AGED, OLD FEMALE STIMULUS PHOTOGRAPHS

\begin{tabular}{|c|c|c|c|c|c|}
\hline$\frac{\text { Component }}{\text { Comparisons }}$ & Mean & $\begin{array}{c}\text { Standard } \\
\text { Error }\end{array}$ & DF & t value & $\mathbf{p}$ \\
\hline \multicolumn{6}{|c|}{ Physical Attributes } \\
\hline $\begin{array}{l}\text { Young/ } \\
\text { 01d }\end{array}$ & $\begin{array}{l}42.6759 \\
44.3704\end{array}$ & 12.3915 & 321 & 1.677 & .095 \\
\hline $\begin{array}{l}\text { Middle/ } \\
\text { 0ld }\end{array}$ & $\begin{array}{l}45.3611 \\
44.3704\end{array}$ & 11.7437 & 321 & .619 & .357 \\
\hline $\begin{array}{l}\text { Young/ } \\
\text { Middle }\end{array}$ & $\begin{array}{l}44.3704 \\
\cdot 5.3611\end{array}$ & 11.1301 & 321 & 1.058 & .291 \\
\hline \multicolumn{6}{|c|}{ Instrumental Qualities } \\
\hline $\begin{array}{l}\text { Young/ } \\
\text { O1d }\end{array}$ & $\begin{array}{r}24.1759 \\
23.9074\end{array}$ & 5.1679 & 321 & 1.401 & .162 \\
\hline $\begin{array}{l}\text { Middle/ } \\
\text { old }\end{array}$ & $\begin{array}{l}23.1389 \\
23.9074\end{array}$ & 6.0473 & 321 & 1.038 & .300 \\
\hline $\begin{array}{l}\text { Young/ } \\
\text { Middle }\end{array}$ & $\begin{array}{l}23.9074 \\
23.1389\end{array}$ & 5.0447 & 321 & .363 & .717 \\
\hline \multicolumn{6}{|c|}{ Interpersonal Qualities } \\
\hline $\begin{array}{l}\text { Young/ } \\
\text { 01d }\end{array}$ & $\begin{array}{l}25.8519 \\
26.7037\end{array}$ & 4.5258 & 320 & 1.276 & .203 \\
\hline $\begin{array}{l}\text { Middle/ } \\
\text { old }\end{array}$ & $\begin{array}{l}25.0093 \\
26.7037\end{array}$ & 5.0628 & 320 & 2.566 & .011 \\
\hline $\begin{array}{l}\text { Young/ } \\
\text { Middle }\end{array}$ & $\begin{array}{l}26.7037 \\
25.0093\end{array}$ & 4.9185 & 320 & 1.293 & .197 \\
\hline \multicolumn{6}{|l|}{ Affect } \\
\hline $\begin{array}{l}\text { Young/ } \\
\text { 01d }\end{array}$ & $\begin{array}{l}17.7315 \\
19.3645\end{array}$ & 6.4643 & 320 & .071 & .9444 \\
\hline $\begin{array}{l}\text { Middle/ } \\
\text { old }\end{array}$ & $\begin{array}{l}17.6667 \\
19.3645\end{array}$ & 7.1290 & 320 & 1.846 & .066 \\
\hline $\begin{array}{l}\text { Young/ } \\
\text { Middle }\end{array}$ & $\begin{array}{l}19.3645 \\
17.6667\end{array}$ & 6.6167 & 320 & 1.775 & .077 \\
\hline \multicolumn{6}{|c|}{ Behavior Predisposition } \\
\hline $\begin{array}{l}\text { Young/ } \\
\text { old }\end{array}$ & $\begin{array}{l}20.3241 \\
20.8611\end{array}$ & $\begin{array}{l}6.7031 \\
6.5858\end{array}$ & 321 & .927 & .355 \\
\hline $\begin{array}{l}\text { Middle/ } \\
\text { 0ld }\end{array}$ & $\begin{array}{l}19.4630 \\
20.8611\end{array}$ & $\begin{array}{l}7.1831 \\
6.5858\end{array}$ & 321 & 1.505 & .133 \\
\hline $\begin{array}{l}\text { Young/ } \\
\text { Middle }\end{array}$ & $\begin{array}{l}20.8611 \\
19.4630\end{array}$ & 6.5858 & 321 & .578 & .564 \\
\hline
\end{tabular}

Number of observations, $\mathrm{N}=72$

$\mathrm{p}<.05 \mathrm{NS}$

$p>.05 \mathrm{~S}$ 
Discussion. In summary, Physical Attributes and Instrumental Qualities components of Physical Attractiveness were mutually agreed upon by both young male and young female subjects in rating Young Female stimulus more positively than either Middle-Aged or $01 \mathrm{der}$ stimuli.

The last age group comparison (Young/Middle-Aged photographs) had t-values that proved significant when examining: Physical Attributes $t(321)=1.058, p>.05$; Instrumental Qualities $t(321)=.363$, p >.05; Interpersonal Qualities $t(320)=1.293, p>.05$; Affect $t(320)=1.775, p>.05 ;$ and Behavior Predisposition $t(321)=.578$, $\mathrm{p}>.05$.

Table XXIV and XXV, reflecting standard error computed on Physical Attributes, appeared to indicate more variability in opinion by the subjects than the other four components. It appears that the standard error showed greater agreement when the subjects evaluated the Young Female stimulus photographs more positively than the Middle-Aged and $01 \mathrm{~d}$ categories.

In mean comparisons by contrasted age groups, the young subjects rated the Young and Middle-Aged photographs more positively in Physical Attributes, whereas the $01 \mathrm{~d}$ stimuli were not rated as high. Contrasting age group means on the four other components (Instrumental Qualities, Interpersonal Qualities, Affect and Behavior Predisposition), the Young and 01d stimulus photographs were rated higher on each of these components compared to the Middle-Aged stimuli as seen on Table XXIV and XXV. In other words, first impression of the Young stimulus photographs' physical attractiveness 
was consistently rated higher on the five components defining physical attractiveness multidimensionally.

The next consistent finding in comparing means was that the oldest people in the stimulus photographs were rated higher than the Middle-Aged photographs in four of the components defining physical attractiveness (Instrumental Qualities, Interpersonal Qualities, Affect and Behavior Predisposition), but were not rated very positively on Physical Attributes.

Analysis of Variance

Tables XXVI and XXVII summarize young male and young female subjects' ratings of female stimuli photographs in all three age groups. A repeated measure, analys is of variance procedure was performed to assess the significance of difference among the five components defined as physical attractiveness.

Findings provide partial support for the first hypothesis. $F$ ratios were significant at the .05 level of significance for Physical Attributes and Instrumental Qualities of the person in the photograph, whereas Interpersonal Qualities, Affect and Behavior Predisposition were not significant.

Young male Subjects rating young, middle-aged, old female stimulus photographs. As reflected on Table XXVI, young subjects rated stimulus photographs positively in all five components across all three age groups. The $F$ ratios for the five components were: Physical Attributes $F(320)=.083, p>.05$ (significant); Instrumental Qualities $F(321)=.12, p>.05$ (significant); Interpersonal Qualities 
TABLE XXVI

YOUNG MALE SUBJECTS RATING YOUNG, MIDDLE-AGED, OLD FEMALE STIMULUS PHOTOGRAPHS

\section{Component}

Source of Variance

SS

df

MS

$\mathrm{F}$

p

Physical Attributes

$\begin{array}{lrrrrr}\text { Between Groups } & 600.3226 & 2 & 330.1613 & 2.503 & 0.0834 \\ \text { Within Groups } & 38368.5266 & 320 & 119.9016 & & \\ \text { Total } & 38968.8491 & 322 & & & \end{array}$

Instrumental Qualities

$\begin{array}{lrrrrr}\text { Between Groups } & 103.5798 & 2 & 51.7899 & 2.166 & 0.1164 \\ \text { Within Groups } & 7676.9755 & 321 & 23.9158 & & \\ \text { Total } & 7780.5553 & 323 & & & \end{array}$

Interpersonal Qualities

$\begin{array}{lrrrrr}\text { Between Groups } & 152.1523 & 2 & 76.0761 & 4.210 & 0.0157 \\ \text { Within Groups } & 5800.8479 & 321 & 18.0712 & & \\ \text { Total } & 5953.0002 & 323 & & & \end{array}$

Affect

$\begin{array}{lrrrrr}\text { Between Groups } & 316.9700 & 2 & 153.4850 & 4.079 & 0.0178 \\ \text { Within Groups } & 12471.3359 & 321 & 38.8515 & & \\ \text { Total } & 17788.3059 & 323 & & & \end{array}$

Behavior Predisposition

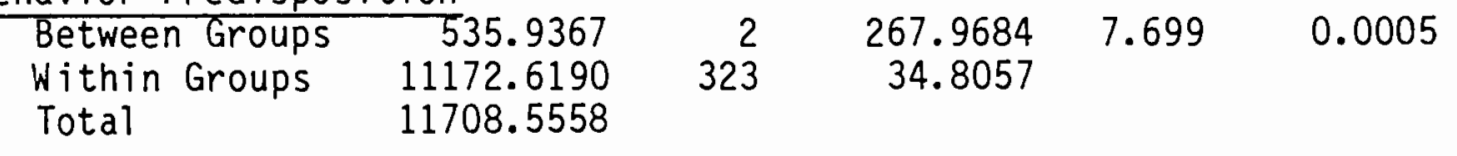

p 3.38 
$F(321)=.02, p<.05$ (not significant); and Behavior Predisposition $F(321)=.0005, p<.05$ (not significant).

Young female subjects rating young, middle-aged, old female

stimulus photographs. Table XXVII indicates young female subjects' ratings of Female stimulus photographs using an analysis of variance to assess the significance of difference among the five components. Examination of this analysis reveals Physical Attributes $F(321)=.24$, $p>.05$ (significant); Instrumental Qualities $F(321)=.35, p>.05$ (significant); Interpersonal Qualities $F(320)=.038, p<.05$ (not significant); Affect $F(320)=.11, p>.05$ (significant); and Behavior Predisposition $F(321)=.32, p>.05$ (significant).

Significant interactions between subjects' gender and physical attractiveness of Young Female Stimuli were found for the components of Physical Attributes among young male subjects $F(322)=2.503, p 3.38$ and young female subjects $F(323)=1.438, \mathrm{p} 3.38$ and the Instrumental Qualities component among young male subjects $F(323)=2.166$. $p 3.38$ and young female subjects $F(323)=1.058, p 3.38$.

Additionally, significant interactions were found between young female subjects and the three other components defined as Physical Attributes $F(323)=1.438, p 3.38$; Interpersonal Qualities $F(322)=3.293, p 3.38$; Affect $F(322)=2.185, p 3.38$; and Behavior Predisposition $F(323)=1.152, p 3.38$.

In contrast to the above data, the young male subjects' ratings showed no significant difference in Interpersonal Qualities $F(323)=4.210, p 3.38$, Affect $F(323)=4.379, p 3.38$ and Behavior Predisposition $F(323)=7.699, p 3.38$. 
TABLE XXVII

YOUNG FEMALE SUBJECTS RATING YOUNG, MIDDLE-AGED, OLD FEMALE STIMULUS PHOTOGAPHS

\section{Component}

Source of Variance

SS

df

MS

$\mathrm{F}$

$\mathrm{p}$

Physical Attributes

$\begin{array}{lrrrrr}\text { Between Groups } & 398.2677 & 2 & 199.1339 & 1.438 & 0.2388 \\ \text { Within Groups } & 44441.7596 & 321 & 138.4478 & & \\ \text { Total } & 44840.0273 & 323 & & & \end{array}$

Instrumental Qualities

$\begin{array}{lrrrrr}\text { Between Groups } & 62.5741 & 2 & 31.2870 & 1.058 & 0.3484 \\ \text { Within Groups } & 9493.6483 & 321 & 29.5752 & & \\ \text { Total } & 9556.2224 & 323 & & & \end{array}$

Interpersonal Qualities

$\begin{array}{lrrrrr}\text { Between Groups } & \text { I54.3097 } & 2 & 77.1548 & 3.293 & 0.0384 \\ \text { Within Groups } & 7497.1389 & 321 & 23.4286 & & \\ \text { Total } & 7651.4485 & 322 & & & \end{array}$

Affect

$\begin{array}{lrrrrr}\text { Between Groups } & 198.6901 & 2 & 99.3450 & 2.185 & 0.1142 \\ \text { Within Groups } & 14549.9980 & 320 & 45.4687 & & \\ \text { Total } & 14748.6881 & 322 & & & \end{array}$

Behavior Predisposition

$\begin{array}{lrrrrr}\text { Between Groups } & \text { To7.4501 } & 2 & 53.7251 & 1.152 & 0.3173 \\ \text { Within Groups } & 14969.4260 & 321 & 46.6337 & & \\ \text { Total } & 15076.8761 & 323 & & & \end{array}$

p 3.38 
Summary. In summary, data gathered from young male subjects rated the Female stimulus photographs higher in the components of Physical Attributes and Instrumental Qualities. Young female subjects rated the same two components higher but considered Affect and Behavior Predisposition equally important. Physical Attributes and Instrumental Qualities of the Young Female stimulus photographs were associated with more positive ratings than the Middle-Aged or 0ld stimulus photographs.

\section{HYPOTHESIS II}

Hypothesis II asserts that young subjects will evaluate the Middle-Aged Male in the stimulus photographs as being more physically attractive than either the Young or 0ld Male stimulus photographs.

\section{Descriptive Statistics}

Results of analyses are reflected in Tables XXVIII and XXIX. They provide a summary of mean comparisons, standard error, and t-values as in Hypothesis I. The table reflects each component defining physical attractiveness.

To simplify for the reader, the Tables and text discussion of the statistical computations once again have been broken into male and female subjects who rated Male stimulus photographs in all three age groups.

Young Male subjects rating young, middle-aged, old male

stimulus photographs. Table XXVIII shows the mean comparisons, standard error and $t$-values among the five components. The 


\section{TABLE XXVIII}

YOUNG MALE SUBJECTS RATING YOUNG, MIDDLE-AGED, OLD MALE STIMULUS PHOTOGRAPHS

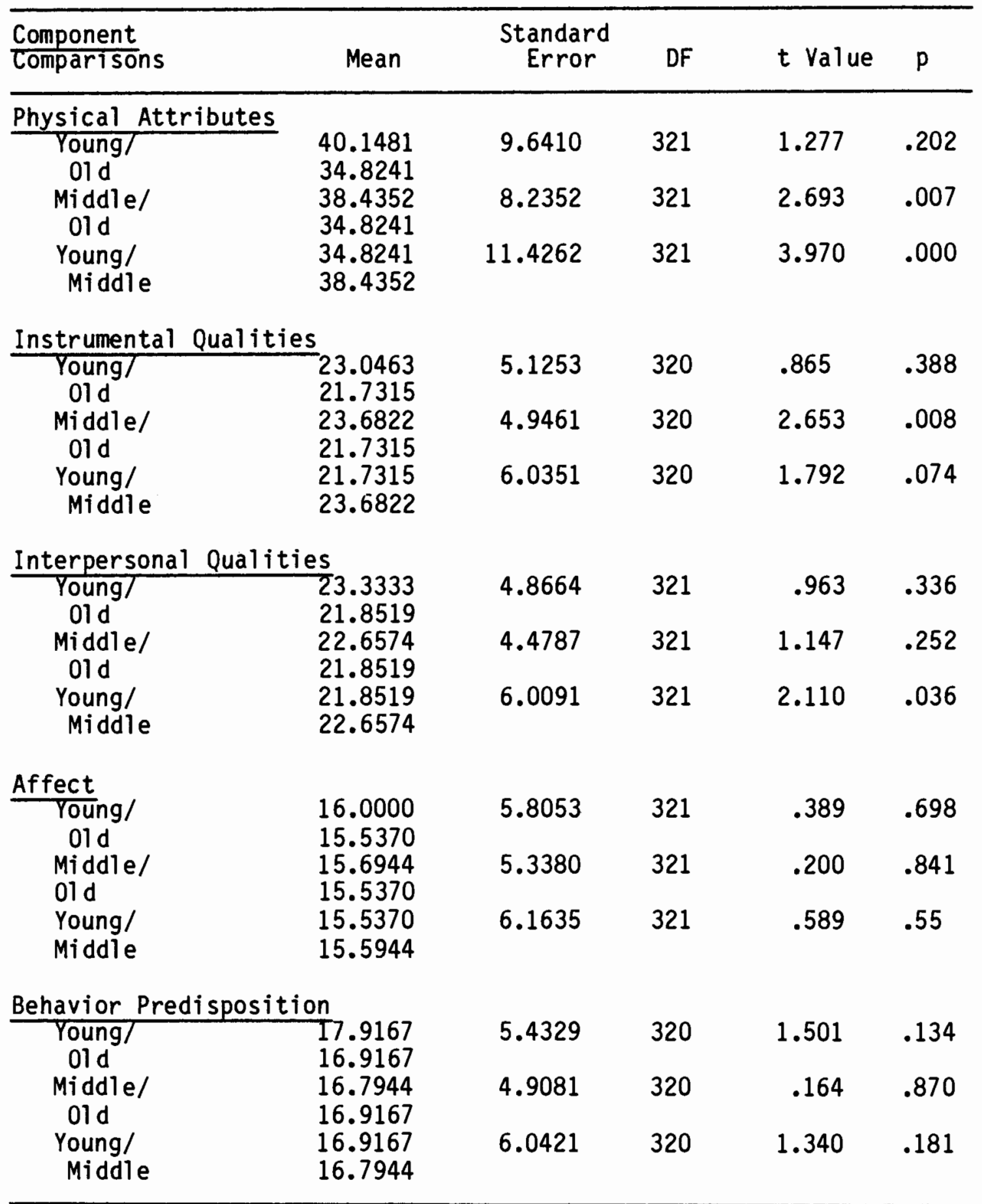

Number of observations, $\mathrm{N}=72$

$p<.05$ NS

$p>.05 \mathrm{~S}$ 
significant t-values for Young/0ld stimuli for the five components of physical attractiveness were: Physical Attributes $t(321)=1.277$, p >.05; Instrumental Qualities $t(320)=.865, p>.05$; Interpersonal Qualities $t(321)=.963, p>.05$; Affect $t(321)=.389, p>.05$; and Behavior Predisposition $t(320)=1.501, p>.05$.

Table XXVII, shows comparisons of Middle-Aged/0ld stimulus photographs rated by young male subjects: Physical Attributes $t(321)=2.693, p=.007$; Instrumental Qualities $t(320)=2.653, p=.008$; Interpersonal Qualities $t(321)=1.147, p>.05$ (significant); Affect $t(321)=.200, p>.05$ (significant); and Behavior Predisposition $t(320)=.164, p>.05$ (significant).

The last comparison of male subjects rating Male stimuli covered Young/Middle-Aged stimulus photographs. The t-values were the following: Physical Attributes $t(321)=3.970, p=.000$; Instrumental Qualities $t(320)=1.792, p>.05$ (significant); Interpersonal Qualities $t(321)=2.110, p>.05$ (significant); Affect $t(321)=.589$, p>.05 (significant); and Behavior Predisposition $t(320)=1.340, p>.05$ (significant).

Young Female subjects rating young, middle-aged, old male stimulus photographs. Table XXIX provides a summary of the young female subjects' ratings of Male stimulus photographs. It illustrates mean comparisons, standard error and t-values in the contrasted age groups. The data presented shows young female subjects' ratings of Young/01d Male stimulus photographs findings on the five components defining physical attractiveness: Physical Attributes $t(321)=4.008, p=.000$; Instrumental Qualities $t(320)=.513$, 
TABLE XXIX

YOUNG FEMALE SUBJECTS RATING YOUNG, MIDDLE-AGED, OLD

MALE STIMULUS PHOTOGRAPHS

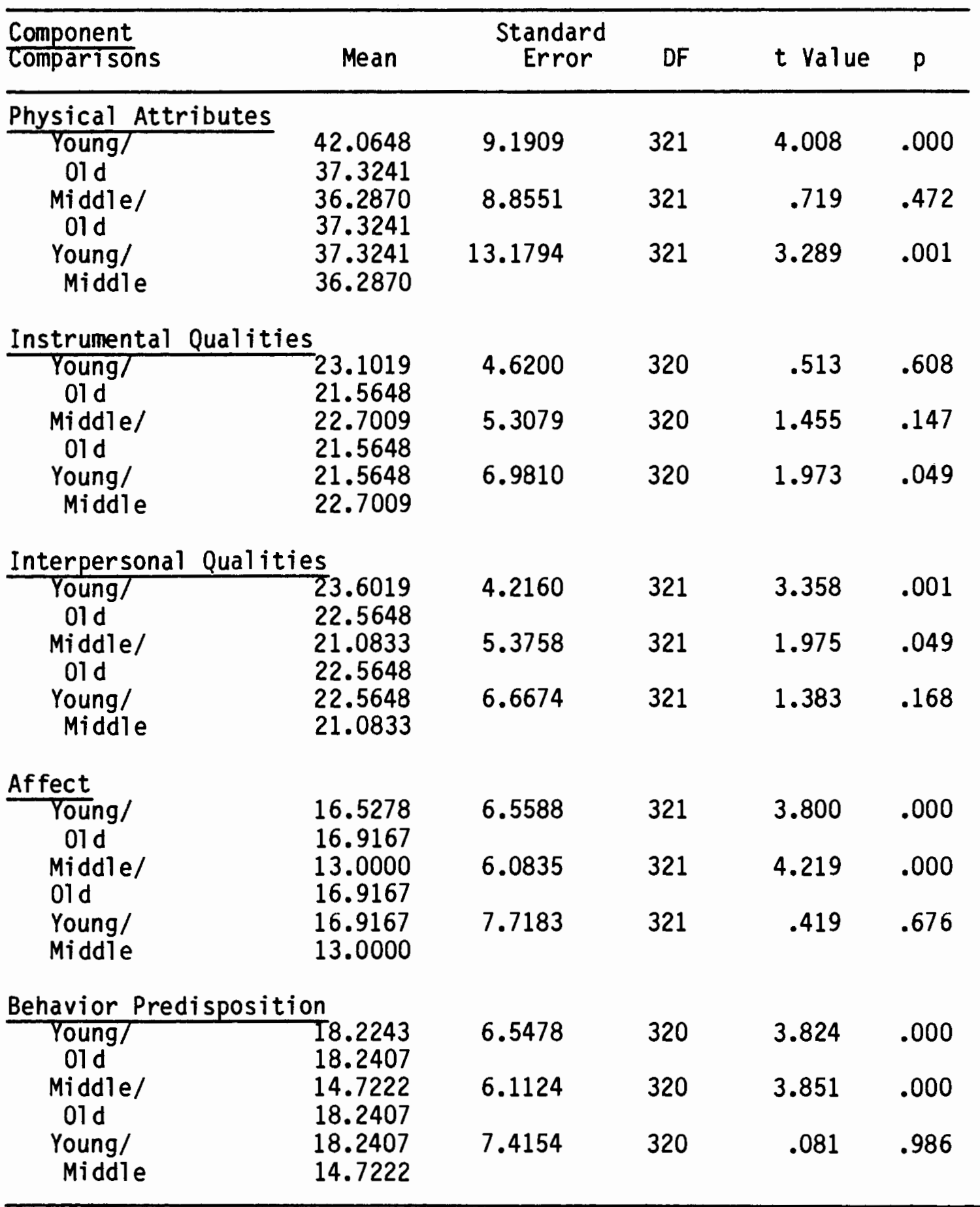

Number of observations, $N=72$

$p<.05$ NS

$p>.05 \mathrm{~S}$ 
$p>.05$ (significant); Interpersonal Qualities $t(321)=3.358, p=.001$; Affect $t(321)=3.800, p=.000$; and Behavior Predisposition $t(320)=3.824, p=.000$.

The subjects' ratings of the Middle/0ld stimulus photographs was Physical Attributes $\mathrm{t}(321)=.719, \mathrm{p}>.05$ (significant); Instrumental Qualities $t(320)=1.455, p>.05$ (significant); Interpersonal Qualities $t(321)=1.975, p=.49$; Affect $t(321)=4.219$, $p=.000$ and Behavior Predisposition $t(320)=3.824, p=.000$.

The last age group comparison on this Table was young female subjects rating Young/Middle-Aged Female stimulus photographs on the following components: Physical Attributes $t(321)=3.289, p=.001$; Instrumental Qualities $t(320)=1.973, p=.049$; Interpersonal Qualities $t(321)=1.383, p>.05$ (signficant); Affect $t(321)=.419, p>.05$ (significant); and Behavior Predisposition $t(320)=.018, p>.05$ (significant).

Discussion. The t-values for Physical Attributes, Instrumental Qualities and Interpersonal Qualities indicate agreement in perceiving the Middle-Aged Male photos more positively than either the Young or $01 \mathrm{~d}$ Male stimulus photographs. Behavior Predispositon t-value did not show agreement in rating the Middle-Aged Male stimulus photographs more positively than either the Young or $01 d$. In summary, the only case where the age group comparisions did not agree in rating Middle-Aged Male stimulus photographs more positively was in the comparison of Young/Middle-Aged Male stimulus photographs.

As summarized on Tables XXVIII and XXIX, the standard error computed on Physical Attributes showed greater variability in opinion 
than the other foor components (Instrumental Qualities, Interpersonal Qualities, Affect and Behavior Predisposition), which showed a greater consensus in rating the Middle-Aged Male stimuli.

In contrasting the means of each age group, the young male subjects rated the Young Males stimulus photographs more positively than the 01 der age group in Physical Attributes. The Middle-Aged Male stimulus photographs were rated higher than the young ones in that component of physical attractiveness. In comparison of the means where young female subjects rated Male stimulus photographs in each category, the Young Male stimulus photographs were rated higher than the 01d Male stimulus photographs in Physical Attributes. It is interesting to note that the young female subjects rated the Young Male stimulus photographs higher than the $01 d$ ones on Physical Attributes and the Middle-Aged Male stimulus photographs were rated lower than the 01 der males.

Young female subjects rated the Young Male stimulus photographs higher than the Middle-Aged Male stimulus photographs. Examination of Tables XVIII and XXIX indicates that young male and female subjects rated the Young Male stimulus photographs higher in Physical Attributes than the Middle-Aged Male stimulus photographs. The Middle-Aged Male stimulus photographs rated slightly lower in this component and $01 d$ Male stimulus photographs were significantly lower in comparisons to the other two age groups.

The Instrumental Qualities component of physical attractiveness mean comparisons within age groups was rated consistently higher by both the young male and young female subjects. In comparison of the 
Young and old stimulus photographs age groups' means, the Young were rated slightly higher than the $01 d$ by both young male and young female subjects. In contrasting the means of both the Young versus Middle-Aged stimulus photographs, the Middle-Aged Males were rated higher than the young ones. It can be inferred from this data that Middle-Aged Male stimulus photographs are rated more positively on the Instrumental Qualities component of physical attractiveness.

The next consistent finding was that young male subjects rated the Young Males more positively in Interpersonal Qualities, with the rest of the age groups falling into descending order, Middle-Aged, then $01 \mathrm{~d}$. The young female subjects rated the Middle-Aged Males lower on Interpersonal Qualities than either the Young or the $01 \mathrm{~d}$.

It is interesting to note that on the Affective component, the young male subjects rated alt three age groups of stimulus photographs as hardly having any difference within contrasted means. In other words, the young male subjects did not see any great differences in Affect between Young, Middle-Aged or 0ld stimulus photographs. Perceived age did not make a difference to the subjects in rating stimulus photographs on the degree they liked the male in all three age groups (Affect component).

Young female subjects rated the Young and $01 \mathrm{~d}$ Male stimulus photographs consistently higher than the Middle-Aged stimulus photographs. Contrary to the male subjects, the young female subjects appeared to have liked the Middle-Aged Males less than either the Young or 01d Male stimulus photographs. 
The next comparison was of the means of the Behavior Predisposition component. These findings almost replicated the findings of the Affect component. Again, the young male subjects rated all three age categories about the same whereas young female subjects rated the Young and 0ld Male stimulus photographs more positively than the Middle-Aged Male stimulus photographs.

It can be postulated that the difference between the young male and young female subjects' responses in both Affect and Behavior Predisposition components were perceptual differences in rating MiddleAged Males, possibly based on society's stereotyping through parents and the mass media.

\section{Analysis of Variance}

A repeated measures analysis of variance procedure was performed to assess the difference among the five components defined as physical attractiveness. Results of the analysis are summarized in Tables XXX through XXXI.

$F$ ratios were not significant at the .05 level of significance for young male subjects rating Physical Attributes of Middle-Aged Male stimulus photographs, whereas the inverse relationship was found at the .05 level of alpha for young female subjects rating MiddleAged Male stimulus photographs in Physical Attributes. The other four components were not significant at the .05 level. Young female subjects consistently rated the Middle-Aged male lower, al though not significantiy lower at the .05 level in all four components. This finding implies that young male subjects do not rate Middle-Aged Male 
stimulus photographs positively in Physical Attribute whereas young female subjects rate the Middle-Aged Male stimulus photographs more positively. It appears that young male subjects rated Middle-Aged males more positively in internal qualities, (Instrumental, Interpersonal, Affect and Behavior Predisposition) than did young female subjects. It is suggested from this finding that young female subjects perceive merely the external Physical Attributes of the MiddleAged Male stimulus photographs.

Young male subjects rating young, middle-aged male and old stimulus photographs. As indicated on Table $X X X$, the $F$ test results of young male subjects rating Male stimuli on the five components were: Physical Attributes $F(321)=.0003, p<.05$ (not significant); Instrumental Qualities $F(320)=.0267, p<.05$ (not significant); Interpersonal Qualities $F(321)=.1090, p>.05$ (significant); Affect $F(321)=.8360, p>.05$, (significant); and Behavior Predisposition $F(320)=.2583, p>.05,($ significant).

Young female subjects rating young, middle-aged and old male stimulus photographs. Table XXXI reflects the analysis of variance on young female subjects rating male stimulus photographs among the five components defined as physical attractiveness. Findings as reflected in the test results were: Physical Attributes $F(321)=.0001, p<.05$, (not significant); Instrumental Qualities $F(320)=.1248, \quad p>.05, \quad$ (significant); Interpersonal Qualities $F(321)=.0037, p<.05$, (not significant); Affect $F(321)=.0000, p<.05$, (not significant); and Behavior Predisposition $F(320)=.0001, p<.05$, (not significant). 
TABLE XXX

YOUNG MALE SUBJECTS RATING YOUNG, MIDDLE-AGED, OLD

MALE STIMULUS PHOTOGRAPHS

\begin{tabular}{|c|c|c|c|c|c|}
\hline $\begin{array}{l}\text { Component } \\
\text { Source of Variance }\end{array}$ & SS & $d f$ & MS & $F$ & $p$ \\
\hline \multicolumn{6}{|l|}{ Physical Attributes } \\
\hline $\begin{array}{l}\text { Between Groups } \\
\text { Within Groups } \\
\text { Total }\end{array}$ & $\begin{array}{r}1595.5226 \\
31171.8333 \\
32767.3560\end{array}$ & $\begin{array}{r}2 \\
321 \\
323\end{array}$ & $\begin{array}{r}797.7613 \\
97.1085\end{array}$ & 8.215 & 0.0003 \\
\hline \multicolumn{6}{|c|}{ Instrumental Qualities } \\
\hline $\begin{array}{l}\text { Between Groups } \\
\text { Within Groups } \\
\text { Total }\end{array}$ & $\begin{array}{r}213.0454 \\
9301.1780 \\
9514.2234\end{array}$ & $\begin{array}{r}2 \\
320 \\
322\end{array}$ & $\begin{array}{r}106.5227 \\
29.0662\end{array}$ & 3.665 & 0.0267 \\
\hline \multicolumn{6}{|c|}{ Interpersonal Qualities } \\
\hline $\begin{array}{l}\text { Between Groups } \\
\text { Within Groups } \\
\text { Total }\end{array}$ & $\begin{array}{l}118.8216 \\
8543.9537 \\
8662.7753\end{array}$ & $\begin{array}{r}2 \\
321 \\
323\end{array}$ & $\begin{array}{l}59.4108 \\
26.6167\end{array}$ & 2.232 & 0.1090 \\
\hline \multicolumn{6}{|l|}{ Affect } \\
\hline $\begin{array}{l}\text { Between Groups } \\
\text { Within Groups } \\
\text { Total }\end{array}$ & $\begin{array}{r}11.9693 \\
10719.7684 \\
10731.7378\end{array}$ & $\begin{array}{r}2 \\
321 \\
323\end{array}$ & $\begin{array}{r}5.9847 \\
33.3949\end{array}$ & 0.179 & 0.8360 \\
\hline \multicolumn{6}{|c|}{ Behavior Predisposition } \\
\hline $\begin{array}{l}\text { Between Groups } \\
\text { Within Groups } \\
\text { Total }\end{array}$ & $\begin{array}{r}81.7085 \\
9617.9765 \\
9699.6849\end{array}$ & $\begin{array}{r}2 \\
320 \\
322\end{array}$ & $\begin{array}{l}40.8542 \\
30.0562\end{array}$ & 1.359 & 0.2583 \\
\hline
\end{tabular}

p 3.38 
TABLE XXXI

YOUNG FEMALE SUBJECTS RATING YOUNG, MIDDLE-AGED, OLD

MALE STIMULUS PHOTOGRAPHS

\section{Component}

Source of Variance

SS $\quad d f$

MS

$F$

p

Physical Attributes

$\begin{array}{lrrrrr}\text { Between Groups } & 2049.5823 & 2 & 1024.7912 & 9.134 & 0.0001 \\ \text { Within Groups } & 36014.3053 & 321 & 112.1941 & & \\ \text { Total } & 38063.8877 & 323 & & & \end{array}$

Instrumental Qualities

$\begin{array}{lrrrrr}\text { Between Groups } & 137.2433 & 2 & 68.6216 & 2.094 & 0.1248 \\ \text { Within Groups } & 10484.8559 & 320 & 32.7652 & & \\ \text { Total } & 10622.0991 & 322 & & & \end{array}$

Interpersonal Qualities

$\begin{array}{lrrrrr}\text { Between Groups } & 346.0735 & 2 & 173.0368 & 5.697 & 0.0037 \\ \text { Within Groups } & 9750.6759 & 320 & 30.3759 & & \\ \text { Total } & 10096.7494 & 322 & & & \end{array}$

Affect

\begin{tabular}{|c|c|c|c|c|}
\hline $\begin{array}{l}\text { Between Groups } \\
\text { Within Groups } \\
\text { Total }\end{array}$ & $\begin{array}{r}1005.7224 \\
14937.1667 \\
15942.8890\end{array}$ & $\begin{array}{r}2 \\
321 \\
323\end{array}$ & $\begin{array}{r}502.8612 \\
46.5332\end{array}$ & 10.806 \\
\hline
\end{tabular}

Behavior Predisposition

\begin{tabular}{|c|c|c|c|c|}
\hline $\begin{array}{l}\text { Between Groups } \\
\text { Within Groups } \\
\text { Total }\end{array}$ & $\begin{array}{r}885.8576 \\
14426.0244 \\
15311.8821\end{array}$ & $\begin{array}{r}2 \\
320 \\
322\end{array}$ & $\begin{array}{r}442.9288 \\
45.0813\end{array}$ & 9.825 \\
\hline
\end{tabular}

p 3.38 
Discussion. The findings discussed above provide partial support for the second hypothesis. In performing the analysis of variance procedure on the five components, a significant interaction between gender and physical attractiveness in the Middle-Aged Male stimulus photographs was discovered in the Instrumental Qualities component. The findings were male subjects $F(322)=3.665, p 3.38$ and female subjects $F(322)=2.094, p 3.38$.

The other four components of physical attractiveness were significant for the male subjects rating the Middle-Aged Male stimulus photographs on the following: Physical Attributes $F(323)=8.215, p 3.38$; Interpersonal Qualities $F(323)=2.32, p 3.38$ and Behavior Predisposition $F(322)=1.359, p$ 3.38. The Affect component of male subjects rating male stimuli was not significant with an $F(323)=.179, p 3.38$.

Interestingly, male subjects rated the Middle-Aged Male more positively than the female in terms of physical attractiveness. No significant interactions were found in female subjects rating the Middle-Aged Male stimulus photographs on Physical Attributes $F(323)=9.134, p 3.38$; Interpersonal Qualities $F(323)=5.697, p 3.38$, Affect $F(323)=10.806, p 3.38$; and Behavior Predisposition $F(322)=9.825, p 3.38$.

\section{HYPOTHESIS III}

Hypothesis III asserts that the more the person in the stimulus photograph is liked (indicated by a high score in Affect), the higher 
young subjects of either gender will rate the stimulus photograph in the other four components.

\section{Pearson $r$ Correlation}

A Pearson $r$ correlation determined the degree of association between Affect and the other four components of physical attractiveness. As reflected on Table XXXII, there appeared to be a moderately high correlation in Physical Attributes $(r=.69)$, Interpersonal Qualities $(r=.59)$, and Instrumental Qualities $(r=.55)$ associated with the Affect component of physical attractiveness at the .001 level of significance. Behavior Predisposition $(r=.91)$ had an extremely high correlation with Affect at the significance level of .001. In other words, the more a person was rated positively in Physical Attributes, Instrumental Qualities, Interpersonal Qualities, and Behavior Predisposition, the more the person would be liked regardless of age.

Summary. Hypothesis III asserted that a high rating in Affect would be mirrored high ratings of the young male and female subjects evaluations in the other four components of physical attractiveness. This hypothesis proved through its highly significant correlations that the five components are an integral part of defining physical attractiveness. 
TABLE XXXII

PEARSON $r$ CORRELATION COEFFICIENTS ON THE AFFECT

COMPONENT ASSOCIATED WITH THE FOUR OTHER COMPONENTS

Component

Physical Attributes

Instrumental Qualities

Interpersonal Qualities

Behavior Predisposition
Pearson $r$ Correlation

.69

.55

.59

.91

$P<.001$

Number of observation, $\mathrm{N}=1,294$

Note: Each Pearson $r$ correlation coefficient was rounded off to the nearest hundredth. 
CHAPTER V

SUMMARY AND LIMITATIONS OF THE STUDY

Introduction

This chapter presents an overview of the thesis prior to discussing the shortcomings of the study. The limitations of the study will indicate problems that may have affected the results of the research design. These are discussed below.

\section{SUMMARY}

The following hypotheses were addressed in this study:

I The Young Female stimuli will be rated more positively in physical attractiveness than either the Middle-Aged or older stimulus photographs by subjects in the 19 to 24 year old age group, (Young).

II Young Subjects will evaluate the Middle-Aged Male in the stimulus photographs as being more physically attractive than either the Young or old Male stimulus photographs.

III The more the person in the stimulus photograph is liked (indicated by a high score in Affect), the higher young subjects of either gender will rate the stimulus photograph in the other four components.

Hypothesis I. Analytic results indicated that both young male and young female subjects rated Young Female stimulus photographs more positively than either Middle-Aged or 0ld Females with respect to the Physical Attributes and Instrumental Qualities components of physical attractiveness. In addition, female subjects rated Young 
female stimuli highest on the components of Affect and Behavior Predisposition.

Hypothesis II. The findings provide partial support for the second hypothesis. Results indicated the t-values for Physical Attributes, Instrumental Qualities and Interpersonal Qualities show agreement in young people perceiving the Middle-Aged Male more positively than either the Young or 0ld Male Stimuli. In contrasting the means for each age group, the male subjects rated the Young Male stimuli higher than the 01d Male group in Physical Attributes whereas the Middle-Aged Males were rated higher than the Young ones in the component of physical attractiveness.

In comparison of the means where young female subjects rated Male stimuli in each category, the Young stimuli were rated higher than the 01d age group in Physical Attributes. In summary, the young female subjects rated the Young Male stimuli higher than the Middle-Aged Male stimuli.

EXamination of Table XXIX and XXX indicates that both young male and young female subjects rated Young Male stimuli higher in Physical Attributes, with the Middle-Aged Male rated slightly lower in this component and 01d Male stimuli still significantly lower in comparison to the other two age groups. It can be inferred from this data that Middle-Aged Male stimuli were rated more positively on the Instrumental Qualities component of physical attractiveness.

The next consistent finding was the young male subjects rated the Young Males more positively in Interpersonal Behavior. Middle-Aged stimulus photographs were rated next highest, and 01d 
were lowest. The young female subjects rated the Middle-Aged Male stimuli lower in Interpersonal Qualities than either the Young or $01 \mathrm{~d}$.

It is interesting to note that on the Affective component, the young male subjects rated all three age groups of stimuli similarly. In contrast to the young male subjects, the young female subjects appeared to have liked (affect) the Middle-Aged Male stimuli less than either the Young or old stimuli.

The next comparison of the means for Behavior Predisposition was almost an exact replication of the findings of the Affect component. Judging by the differences between the young male and young female subjects' responses, it can be postulated that based upon our gender conditioning in society, both Affective and Behavior Predisposition components were perceived differently in relationship to Middle-Aged Male stimuli.

A very interesting finding of the study was that young male subjects rated Middle-Aged Males more positively in internal qualities (such as Instrumental Qualities, Interpersonal Qualities, Affect, and Behavior Predisposition) than did young female subjects. The findings suggest that young female subjects perceive merely the external Physical Attributes of the Middle-Aged stimuli.

Hypothesis III. The results indicated a significant relationship between Affect and the other four components of physical attractiveness. The more a person was rated positively by young subjects in Physical Attributes, Instrumental Qualities, Interpersonal Qualities and Behavior Predisposition, the more the person would be liked regardless of age. There was a high degree of 
association between Affect and Physical Attributes, Instrumental Qualities, Interpersonal Qualities and Behavior Predisposition. This finding supports the investigator's perspective of examining physical attractiveness multidimensionally rather than as a "unidimensional" concept. Physical attractiveness has many components, each of which can be assessed at different levels.

\section{LIMITATIONS OF THE STUDY}

Shortcomings exist in any research design, no matter how many precautions or how much forethought an investigator exerts in attempting to avoid them. The limitations of this study included sample size and homogeneity of the sample. The fact that the situation for judging was artificial may have created a barrier in how some participants responded in the study. Some participants indicated familiarity with some of the faces of the stimulus photographs. This may have affected how these subjects responded. Also, some indicated a reluctance to evaluate the stimulus photographs with positive or negative evaluations for fear that the information might become "public knowledge." All of these shortcomings of the study are discussed below in greater detail.

\section{Overall Size of the Study}

This study was a composite of three research studies rolled into one. In addition to the main research hypothesis, two other aspects were addressed. The first one was creating the standardization of photo orders consisting of three physical attractiveness intensity levels. The second one was constructing, 
testing and retesting a questionnaire of physical attractiveness called the OFIQ. This thesis could very well have examined merely one part of this study rather than all three. As a consequence, the investigator was forced to extend the length of time taken to complete the study. Because people's opinions change, the element of "time" could have affected the results of the study. What is considered physically attractive to a person is subjective, not objective, and may vary from year to year.

Sample Size and Homogeneity

Generalizability of the study was limited by the fact that the entire population sampled consisted of Caucasian and college-educated or college-enrolled individuals between the ages of 19 to 24 .

Young Caucasian and college-educated people do not make up the entire world. Therefore, the population sampled was not representative except for that specific group of people. Furthermore, the number of people sampled (interviewed) was small in Sections I, II, III, and IV (refer to Chapter III).

Section I: The total number of stimulus photographs was reduced from 48 to 18 . There were three males and three females representing high, medium and low physical attractiveness intensity levels. There was one photograph from each age group that represented very physically attractive (high), neutral (medium) and very physically unattractive (low). The number of stimulus photographs was too few in each intensity level to be conclusive about the findings of this study, but the results of this study are heuristic for futher research. 
Section II: A total number of 18 selection judges, consisting of three males and three females within each age group, helped to reduce the number of stimulus photographs. The total number of selection judges are few. This may have caused a lack of representativeness in the results of the study. In order to have a small representative sample, the number of participants should be larger.

Section III: Six young and six old questionnaire judges rated one of three photo orders using the OFIQ. In this phase, the main unit of analysis was developing the questionnaire, testing, retesting and modifying it. Even though the questionnaire judges were few, the number of $i$ tems on the OFIQ totalled 105 items, which was a representative number of items sampled.

As indicated earlier, the make up of the six questionnaire judges omitted the Middle-Aged group, so items selected for the questionnaire may not reflect all age groups, because a person may evaluate his or her own age group differently than he or she would another age group.

Section IV: Seventy-two subjects represented the Young group. The extent to which the Middle-Aged and 01 der age group were represented for evaluating the stimulus photographs remains unknown. The generalizability of the findings based upon the younger age group of subjects is probably not the same as for the middle-aged and older subjects. It has been suggested by some geronotologists that younger and older adults are more similar to each other than middle-aged adults due to "cohort" or "life stage" differences (Hendricks and Hendricks, 1977; and Cain, 1975, 1968, 1967). The use of middle-aged 
subjects, employing the same procedures as indicated in this study with younger subjects, might be significant in this field of research, as would using middle-aged and 01 der subjects for comparison with the younger subjects' evaluations of stimulus photographs' physical attractiveness.

Context of the Situation

The standardized environment at Portland State University may have affected the responses of the participants evaluating the stimuli by helping them perceive the judging situation as "not real" or a "superficial relationship" created in a laboratory setting. One of the major criticisms about physical attractiveness and interpersonal relationships research is that they do not exist in isolation but are influenced by the context of the situation such as school, work or play.

Additionally, the campus setting where the participants were interviewed by the investigator might have been uncomfortable or distracting. This may have varied their responses to some degree. All speech communication, whether it is intrapersonal, interpersonal or in groups, is influenced by the physical setting and social context in which it occurs. The context of the situation remains unaccounted for except by the investigator's attempt to standardized the environment. The investigator controlled the environment by standardizing the testing room and presenting pre-test instructions that did not vary. 
Participants' Reluctance to Respond

Some participants expressed a reluctance to describe positive and negative physical characteristics of the person in the photograph. Participants were asked the following two questions:

(1) What is it about that person you find very physically attractive?

(2) What is it about that person you find very physically unattractive?

The investigator assured each participant that all information obtained on the tape-recordings would be confidential. The responses of the selection judges may still have been biased by this reluctance. The younger judges were more "verbal" and "outspoken" of the stimuli's physical characteristics whereas the middle-aged and older judges were much more reserved in giving their opinions. Some of the middle-aged and older participants expressed concern that their positive or negative remarks might get back to the person in the photograph or become public knowledge.

Many older participants said they were afraid to comment about the person's negative Physical Attributes, feeling it might be held or used against the person in the photograph. The older judges expressed particular discomfort at being critical of the stimulus physical appearance, for fear that if they were in reverse roles, the person in the photograph would do the same to them.

This appeared to be a comment frequently mentioned by the older age group rather than the younger or middle-aged group. Gerontological researchers have indicated that attitudes toward the 
aged are deeply rooted in one's own self-image (Kahana, 1970). Perhaps, because the young have difficulty visualizing themselves as old, the younger age group were more out-spoken in identifying negative physical characteristics of the stimuli.

Participant being Familiar with the Faces of the Stimulus Photographs

Another consideration is that all the participants may have different evaluations of the stimulus physical attractiveness depending on whether he/she thinks the person looks "familiar" or "strange." Particular precaution was taken by the investigator to avoid "familiarity" of the stimulus. The investigator had a question on each questionnaire that read: "Do you know or have you seen this person in the photograph before?" Even though this safety measure was taken, some participants remarked after the interview was completed that a given stimulus looked like a person they knew. In this respect, all bias was not totally eliminated but could be included as part of the "subjective opinion" in this study. 


\section{CHAPTER VI}

\section{IMPLICATIONS AND APPLICATIONS}

This chapter presents the thesis' analytical results and their generalizability to applications in the "real world." It will address the findings and apply them to potential real-life problems.

Prior research has found that younger females appear more physically attractive, desirable, and likeable. Little research has been done examining physical attractiveness across generational groups in an attempt to make the above statements more conclusive. Prior studies are very subjective in nature, not anchoring down any concrete variables that assess physical attractiveness. This research study virtually stands alone in examining physical attractiveness multidimensionally in order to learn--empirically and objectively--what makes a young, middle-aged or older person physically attractive.

Most of the prior research conducted on physical attractiveness has not assessed its many components. Rather, past investigators have perceived physical attractiveness as a "unidimensional" concept. This study was significant because of the development and construction of the questionnaire. Prior researchers have not examined physical attractiveness multidimensionally with a comprehensive questionnaire based upon an "attitude", (see Chapter I, p. 21). 
Another reason this study is a significant contribution to the literature of physical attractiveness is that it attempts to devise a systematic order for the presentation of the stimuli. Earlier studies have examined stimulus photographs' physical attractiveness with high, medium, or low intensity levels (Nordholm, 1980; Stroebe, Insko, Thompson and Layton, 1971; Miller, 1970 and Dion, Berscheid, and Walster, 1972) but few studies have controlled the given order of the stimuli by their intensity level using a mathematical matrix. In this study, a mathematical matrix was "divided" into six photo orders using the combination of the three photo orders from Section III (refer to Chapter III). The matrix was used to create physical attractiveness intensity levels based upon the selection judges' evaluations of the stimulus photographs' physical attractiveness.

The methodological procedures of prior studies on physical attractiveness need to be re-evaluated in order to address apparent contradictions in their findings. Currentiy no one has examined the reliability of "photographs" versus "live subjects" as stimuli. The inadequate research methods in this area have helped accumulate an enormous amount of subjective information that lacks the scientific approach.

Many studies have used photographs as stimulus materials in less scientific ways, to assess physical attractiveness or behavior attraction (Bryne, London, and Reeves, 1968; Dion, Berscheid and Walster, 1972; Miller, 1970; Perrin, 1921 and Krebs and Adinolfi, 
1975). Other studies (Brislin and Lewis, 1968; Bryne, Ervin and Lamberth, 1970 and Walster, Aronson, Abrahams, and Rottman, 1966) have allowed subjects short contact in an artificial situation (such as a computer date) with their stimulus materials (i.e., a real person) and almost all the studies have restricted their research to members of the opposite sex (in comparison to people of the same sex). It would also be interesting to speculate upon how individuals perceive physical attractiveness from a homosexual perspective. Additionally, future research might examine physical attractiveness ratings when subjects and stimulus are paired in terms of similar physical attractiveness. Such pairings could have significant effects upon the subjects' first impressions of the stimulus photographs' attractiveness. In other words, would a physically attractive person perceive another physically attractive person differently than an average looking person evaluating a physically attractive person? This would be an interesting question to address in a future study.

Few studies have controlled or manipulated both the subjects' and the stimuli's physical attractiveness intensity levels. Hendrick and Taylor (1968) and Stroebe, Insko, Thompson and Layton (1971) have manipulated attitude similarity using only a survey instrument, rather than using two different sets of judges to validate the stimulus photographs' physical attractiveness ratings, as this investigator did. 
Another methodological question that could be examined would be whether or not there is a significant effect if a scale of seven to one is used as compared to a scale of one to seven. Currently, this question has not been explored. It may or may have a profound effect upon interpretation of previous research conducted on physical attractiveness.

According to Butler (1975) ageism refers to the "process of systematically sterotyping and discriminating against people because they are old." This study was significant for addressing physical attractiveness across age groups, in a youth-oriented society that practices ageism. The subtle stereotypes in American mass media are influential in supporting and maintaining ageist attitudes.

Advertising also sets cultural norms of attractiveness that condition even small children to know innately what is and is not considered physically attractive.

Early on physical attractiveness is a very strong influence on first impressions and the ensuing expectations we place upon strangers. Stereotypes of ageism and attractiveness go hand in hand in the visual mass media to the extent that individuals'evaluations of physical attractiveness are often influenced by one's video idols. We tend to determine a person's age from his or her physical appearance. Ironically, many older people are trying to stay younger-looking while many teenagers are in a hurry to grow up 
and look older. Chronological age has been judged by looks in the past with some degree of accuracy but now, with so many clever cosmetic practices, it is very easy to disguise one's age.

According to recent studies (Baum and Boxley, 1983 and Markides and Boldt, 1983), older people perceive themselves as being younger than their actual chronological age. Society loads the label "old" with negative stereotypes. Prior studies have shown that people age 65 and older consider themselves "middle-aged," not "07d," despite the fact that society defines this particular passage of life (Markides \& Boldt, 1983 and Baum \& Boxley, 1983) as "retirement age". Perhaps in time, with the increased older population and the extended average life expectancy, American society will change its chronological definition of "old age." If attitudes toward the elderly are to be improved or changed, a significant effort needs to be made to re-educate all age groups of the population and to avoid treating the aged as a select population but rather to look at old people as individuals. If how we feel individually about a person effects how we relate or interact with them, then society's view of the elderly must effect the life style of the aged.

Other minority groups have found that re-education through the mass media can help to overcome cultural prejudices. In recent years, the media coverage for the women's movement has played a significant part in making people more aware of the cultural roles 
they once accepted without question and to confront issues such as equal pay. The phrase "black is beautiful" shows how re-education through the media can help to overcome negative self-images. It will be many years to come before we hear jingoism such as "gray is great" or "wrinkle power." Some of the myths about aging need to be brought to the attention of our society.

Al though this study spawned more questions than it answered, we can better understand our own subtle non-verbal communication cues by looking at others. By examining current literature and the findings of this study, we may understand how our attitudes about physical attractiveness and first impressions have developed. This increased awareness may help us to remove some of the classic stereotypes about attractiveness that prevail in our society today; stereotypes that will become less and less acceptable to the increasingly gray population of tomorrow. 
Adams, G.R. \& Huston, T.C. (1975). Social perception of middle-aged persons varying in physical attractiveness. Developmental Psychology, 1975, 11(5), 657-658.

Baum, S.K. \& Boxley, R.L. (1983). Age identification in the elderly. The Gerontologist, 23(5), 532-537.

Berscheid, E. \& Walster, E.H. (1969). Interpersonal attraction. Reading, MA: Addison-Wesley Publishing Company.

Berscheid, E. \& Walster, E.H. (1978). Interpersonal Attraction. (2nd ed.). Reading, MA: Addison-Wesley Publishing Company.

Berscheid, E. \& Walster, E. (1974). Physical attractiveness. In $L$. Berkowitz (Eds.), Advances in experimental social psychology (Vol. 7). New York: Academic Press.

Berscheid, E., Walster, E., \& Campbell, R. (1972). Grow old along with me. Mimeograph copies from the authors.

Berscheid, E., Dion, K.K., Walster, G. and Walster, G.W. (1971). Physical Attractiveness and dating choice: a test of the matching hypothesis. Journal of Experimental Social Psychology, $\underline{7}, 173-189$.

Birdwhistell, R.L. (1970). Kinesics and context. Philadephia: University of Pennsylvania Press.

Brown, J. K. (1982). Cross-cultural perspectives on middle-aged women. Current Anthropology, 23(2), 143-156.

Brislin, R.W. and Lewis, S.A. (1968). Dating and physical attractiveness: Replicated. Psychological Reports 22, 976.

Bryne, D., London, 0. \& Reeves, K. (1968). The effects of physical attractiveness, sex, and attitude similarity on interpersonal attraction and personality. Journal of Personality, 36 , 259271.

Bryne, D., Ervin, C.R. \& Lamberth, J. (1970). Continuity between the experimental study of attraction and "real life" computer dating. Journal of Personality and Social Psychology, 16 157-165. 
Burgoon, J.K. \& Saine, T. (1978). The Unspoken Dialogue. Boston, MA: Houghton-Mifflin.

Butler, R.N. (1975). Why survive? Being old in America. New York: Harper.

Cain, L.D. Jr. (1968). Aging and the character of our times. Gerontologist, $8,250-258$.

Cain, L.D. Jr. (1967). Age status and generational phenomena: the new old people in contemporary America. Gerontologist, 2, 250-258.

Cain, L.D. Jr., \& Wilson, K.B. (1979). Implementation of the age discrimination act of 1975 . Revised version of a paper presented to the annual meeting of the Gerontological Society, Washington, D.C.

Cavior, N. (1970). Physical attractiveness, perceived attitude similarity, and interpersonal attraction among fifth and eleventh grade boys and girls. Doctoral dissertation, University of Houston.

Cavior, N. \& Dokeck, P.R. (1971). Physical attractiveness selfconcept: a test of Mead's hypothesis. Proceedings of the 79th Annual Convention of the American Psychological Association, $\underline{6}$, 319-320.

Cross, J.F. \& Cross, J. (1971). Age, sex, race and the perception of facial beauty. Developmental Psychology, $5,433-439$.

Dillman, Don A. (1978). Mail and telephone surveys: The total design method. New York: John Wiley and Sons, Inc.

Dion, K.K., \& Berscheid, E. (1974). Physical attractiveness and peer perception among children. Sociometry, 37(1), 1-12.

Dion, K. Berscheid, E. \& Walster, E. (1972). What is beautiful is good. Journal of Personality \& Social Psychology, 24 (3), 285290.

Ekman, P. \& Friesen, W.V. (1969). The repertoire of nonverbal behavior: Categories, origins, usage and coding. Semiotica, 1 , 49-98.

Ekman, P. \& Friesen, W.V. (1972). Hand movements. Journal of Communication, 22, 353-374.

Ekman, P. (1976). Movements with precise meanings. Journal of Communication, 26, 14-26. 
Helson, H. (1964). Adaptation-level theory: an experimental and systematic approach to behavior. New York: Harper.

Hendrick, C. \& Hendrick S. (1983). Liking, loving, and relating. Monterey, CA: Brooks/College Publishing Co.

Hendricks J. \& Hendricks, C.D. (1977). Aging in mass society: myths and realities. Cambridge, MA: Winthrop Publishers, Inc.

Huston, T. L. (1973). Ambiguity of acceptance, social desirability and dating choice. Journal of Experimental Social Psychology, $\underline{9}(1), 32-42$.

Johnson, D. (1984). Attribution, attractiveness, stereotypes and the elderly. Developmental Psychology, 20(6), 1168-1172.

Kaat G.R. \& Davis, K.E. (1970). The dynamics of sexual behavior of college students. Journal of Marriage and the Family, 32 , 390-399.

Kagan, J. (1964). Acquisition and significance of sex role identity. In M. Hoffman and L. Hoffman (Eds.) Review of Child Development Research, (Vol. 1). New York: RussetT Sage.

Kahana, B. (1970). 01d age seen negatively by old as we 11 as young: Geriatric Focus, 9, 1113.

Katz, D. \& Scotland, E. (1959). A preliminary statement to a theory of attitude structure and change. In Skoch (Ed.), Psychology: A study of a science (Vol 3). New York: McGraw-HiT1, 19-59.

Knapp, M.L. (1980). Essentials of nonverbal communication. New York: Hoit, Rinehart and Winston.

Knapp, M.L. (1972). Nonverbal communication in human interaction. New York: Holt, Rinehart and Winston, Inc.

Knapp, M.L., Wiemann, J.M. \& Daiy, J.A. (1978). Nonverbal communication: issues and appraisal. Human Communication Research, 4, 271-280.

Krebs, D. and Andinolfi, A. (1975). Physical attractiveness, social relations, and personality style. Journal of Personality and Social Psychology $31(2), 245-253$.

Lerner, R.M. \& Geller, E. (1969). Body build identification, preference and aversion in children. Developmental Psychology, 1 , 456-462.

Levinger, G. \& Snoek, J. D. (1972). Attraction in relationships: a new look at interpersonal attraction. Morristown, New Jersey: General Learning Press. 
Markides, K.S. \& Boldt, J.S. (1983). Change in subjective age among the elderly: a longitudinal analysis. The Gerontologist, $\underline{23}(4), 422-427$.

Marlowe, D. \& Gergen K.J. (1969) Personality in interactions. In G. Lindzey \& E. Aronson (Eds.), Handbook of Social Psychology (Vol. 3), Reading, MA: Addison-Wesley.

Miller, A. (1970). Social perception of internal-external control. Perceptual \& Motor Skills, 30, 103-109.

Miller, A.G. (1970). Role of physical attractiveness in impression formation. Psychonomic Science, 19(4), 241-242.

Mortensen, C.D., Arnston, P.H. \& Lustig, M. (1977). The measurement of verbal predispositions: scale development and application. Human Communication Research, $\underline{3}(2), 146-158$.

Murstein, B.I. (1972). Physical attractiveness and marital choice. Journal of Personality and social Psychology, 22(1), 8-12.

Nie, N.H., Hull, C.H., Jenkins, J.G., Steinbrenner, K. \& Bent, D. H. (1970). SPSS (Statistical package for the social sciences) (2nd ed). New York: McGraw-Hi il Book.Company.

Nordholm, L:A. (1980). Beautiful Patients are good patients: evidence for the physical attractiveness stereotype in first impressions of patients. Social Science \& Medicine, 14(1), 81-83.

Perrin, F. (1921). Physical attractiveness and repulsiveness, Journal of Experimental Psychology, 4, 203-217.

Petersen, M. (1976). Aging and the semantic differential. Unpublished thesis, Portland State University.

Rosen, G.M. \& Ross, A.0. (1968). Relationship of body image to self-concept. Journal of Consulting and Clinical Psychology, $\underline{3}(1), 100$.

Rosenberg, M.J. \& Hovland, C. (1960). Cognitive, affective, and behavioral components of attitudes. In C.I. Hovland \& M.J. Rosenberg (Eds.) Attitude Organization and Change. New Haven, Yale University Press.

Ryder, N.B. (1968). Cohort analysis. In D. L. Sills (Ed.), International Encyclopedia of the Social Sciences (Vol. 2). New York: Free Press.

Ryder, N.B. (1965). The cohort as a concept in the study of social change. American Sociological Review, 30, 843-861. 
Secord, P.F. \& Jourard, S.M. (1953). The appraisal of body-cathexis: body-cathexis and the self. Journal of Consulting Psychology, 17, 343-348.

Siegman, A.W. \& Feldstein, S. (1978). Nonverbal behavior and communication. Hillsdale; N.J.: Lawrence Erlbaum.

Sontag, A.T. (1980). The double standard of aging. In Annual Editions: Aging (2nd ed.) Guilford, CT: Dushkin Publishing Group, Inc.

Stroebe, W., Insko, C.A., Thompson, V.D. \& Layton, B.D. (1971). Effects of physical attractiveness, attitude similarity, and sex on various interpersonal attraction. Journal of Personality and Social Psychology, 18, 79-91.

Tabor, E. (1970). Decoding of consistent and inconsistent attitudes in communication. Doctoral dissertation, Illinois Institute of Technology.

Thibaut, J.W. \& Kelly H.H. (1965). The social psychology of groups. New York: Wiley.

Udry, R.J. (1965). Structural correlates of feminine beauty preferences in Britain and the United States: A comparison. Sociology Social Research, 49 (3), 330-342.

Walster, E., Aronson, V., Abrahams, D. \& Rottman, L. (1966). Importance of physical attractiveness in dating behavior. Journal of Personality \& Social Psychology, 4 (5), 508-516.

Weiner, M., Devoe, J., Rubinow, S. \& Geller, J. (1972). Nonverbal behavior \& nonverbal communication. Psychological Review, 79, 185-214.

Williams, F. (1974). Reasoning with statistics. (2nd ed.). NY: Holt, Rinehart and Winston.

Zimbardo, P.G., Ebbesen, E.B. \& Maslach C. (1977). Influencing attitudes and changing behavior: an introduction to method, theory, and applications of social control and personal power. (2nd ed). Reading, MA: Addison Westey Publishing Company. 
APPENDIX A

RECRUITMENT NOTICES 
Recruitment Notice: Stimuli

\section{SIGN - UP SHEET}

Jewel Hunter is doing a $\mathrm{class}$ project on Interpersonal Communication and needs you to volunteer to have a $5 \times 7$ color snap shot taken. The photographs will be used to do research on first impressions people have of each other. The ages needed for this study are the following: 1) $19-24,2) \quad 42-47$, and 3) 65-70 years old. She will contact you to arrange a specific time for you to have your picture taken by a professional photographer. All the photographs will be taken at Portland Community College, Sylvania Campus, Instructional Material Production Center, CC (College Center), Room A8.

All the information in this study will be strictly confidential and your name will not appear on any of the findings. Upon the completion of this study, you will receive a copy of the abstract report of the research findings you have helped make possible by participating. In addition, you will receive a free $5 \times 7$ color photograph of yourself upon request. Your efforts and participation in this project are greatly appreciated. If you are interested, please sign up below and she will contact you as soon as possible. Thank you.

PLEASE PRINT!

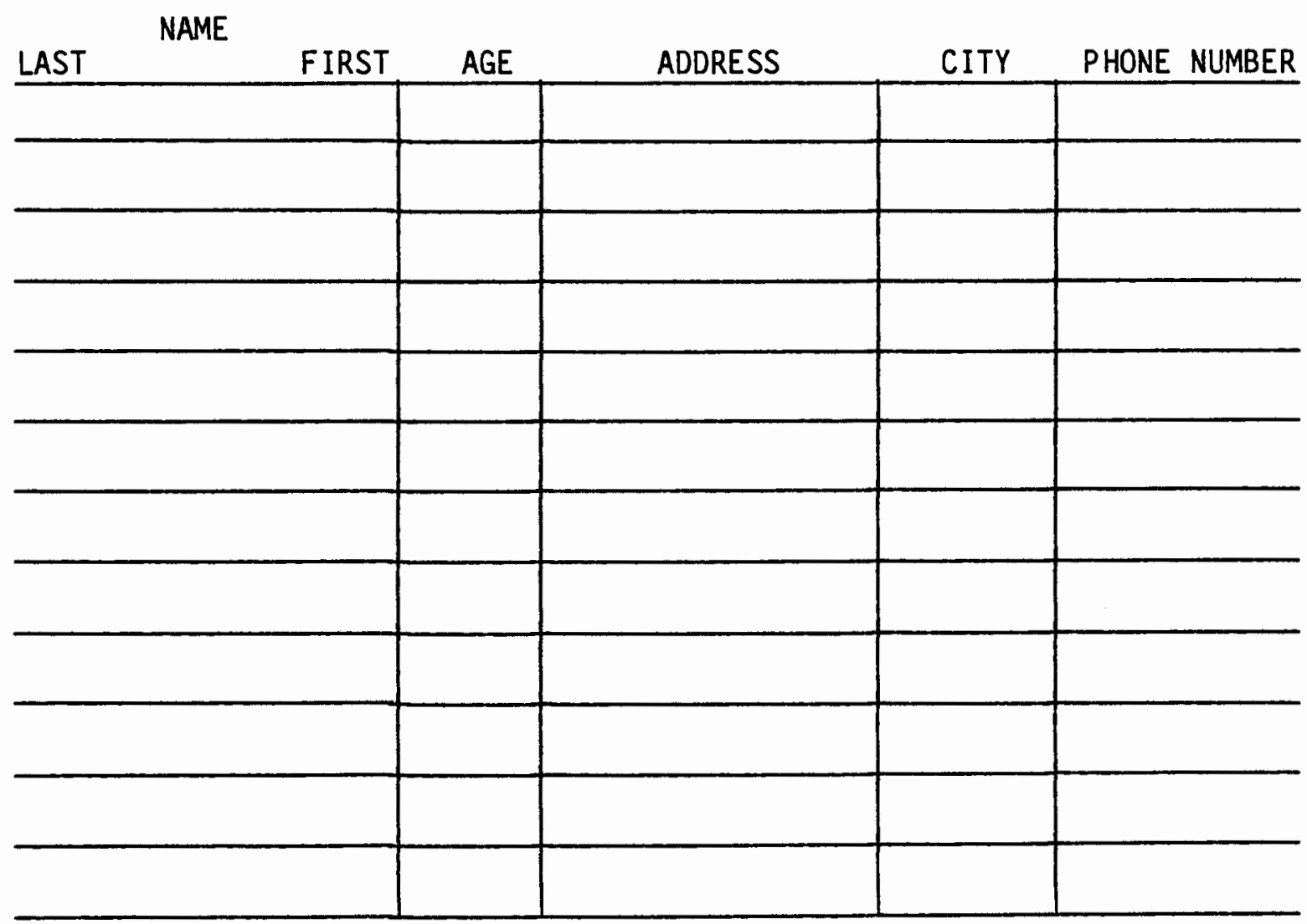




\section{RECRUITMENT NOTICE: PHOTO AND SELECTION JUDGES \\ FIRST IMPRESSIONS ARE THE MOST IMPORTANT AND LASTING}

Jewel Hunter is doing a class project on "Interpersonal Communication" and needs you to volunteer to give your impression of the person in a number of photographs. Your efforts and participation in this project are very valuable and would be greatly appreciated. This project can not take place without your help!

The findings of this study must be gathered before the end of Spring term. Your participation is needed for a MAXIMUM OF ONE HOUR. She will contact you when the exact time and location on campus at Portland State University has been determined.

If you are interested in participating in this study please give your name, address and phone number to Judy Pond at the Adult Learning Center, Cramer Hall 124 (229-4739). All the information in this study wil be held strictly confidential and your name will not appear on any of the findings.

If you are not interested but know of someone who might be, please let them know about this study and either give their names and phone numbers to Judy Pond or have them call at the above number.

Thank you. 
RECRUITMENT NOTICE: QUESTIONNAIRE JUDGES

'INTERPERSONAL COMMUNICATION'

Jewel Hunter is doing a thesis on "A Multidimensional Analysis of Physical Attractiveness in the Formation of First Impressions" and needs you to volunteer to give your first impression of the person in a number of photographs. If you are between the ages of 19 to 24 years and have the maximum of one hour to contribute to a worthwhile cause, then contact her at 245-5422 to schedule an appointment or to find out more information. Starting today through August 27th appointments and interviews wil be conducted from $8 \mathrm{a} . \mathrm{m}$. to $9 \mathrm{p.m}$. and on week ends at the Institute on Aging, Portland State University. The Institute on Aging is located in the Francis Manor Building, Room 104

614 S.W. Montgomery St. (S.W. Broadway and S.W. Montgomery St.)

For appointment cancellation or rescheduling call 229-3952 and leave a message if she is not there.

Until the study has been completed the purpose of the study will have to remain undisclosed so that the results will not be biased. All information will remain strictly confidential and your name will not appear on any of the findings. Upon completion of the study, she will mail to you a report of the results that you have helped to make possible through your willingness to participate. 
RECRUITMENT NOTICE： SUBJECTS

HELP! HELP! HELP!

Jewel Hunter is a graduate student at Portland State University in the Gerontology and Speech Communication Program. She is doing her thesis on "A Multidimensional Analysis of Physical Attractiveness in the Formation of First Impressions" and needs you to indicate your first impressions of people shown in a series of photographs. The interview takes approximately one hour and she would schedule an interview at this location. Jewel needs 36 men and 36 women to participate in her study to graduate from the Masters of Science Program. This is not a test of you personally, nor will any of these answers be identified as belonging to particular individuals. Your answers will be kept strictly confidential.

You are not obligated to participate in the study. If you are interested in calling her to find out more information regarding what you would be doing as a participant in this study, then contact her at 245-5422 or leave a message at 229-3952. At the front desk, there is a sign-up sheet where you can leave your name and phone number so Jewel Smith can contact you. Upon completion of the study she will mail you a report of the results that you have helped to make possible through your willingness to participate. 
APPENDIX B

CONSENT FORMS 


\section{CONSENT FORM: STIMULI}

I hearby give authorization and grant permission for my photograph to be utilized in the study, "A Multidimensional Analysis of Physical Attractiveness in the Formation of First Impressions," conducted by Jewel Hunter. I understand that the purpose of this study is to learn more about interpersonal communication and first impressions.

I understand that my participation in the study involves being photographed and answering some biographical questions. It has been explained to me that my name and identifying personal information will be kept strictly confidential and that the information collected will be used for research and educational purposes only. The amount of time needed for my participation will be approximately five minutes. Jewel Hunter will be present while the photograph is taken so she can answer any questions I may have pertaining to the study or my participation.

I understand that upon completion of this study I will receive an overview report of the research findings. It is further understood that I can withdraw from participation in this study at any time. I have read and understood the above information and I give my consent to participate in the study.

DATE : NAME (Printed):

SIGNATURE:

I hereby authorize Jewel Hunter to print my photograph in a national research publication. (CHECKONE)

$\square$ YES, I CONSENT.

$\square$ NO, I DO NOT CONSENT.

I give my consent to Jewel Smith and other researchers to use my photograph and biographical information for purposes of non-profit research. (CHECK ONE)

$\square$ YES, I GIVE MY PERMISSION.

NO, I DO NOT GIVE MY PERMISSION 


\section{CONSENT FORM: PHOTO AND SELECTION JUDGES}

I hearby give authorization and grant permission for participating as a judge in this study, "A Multidimensional Analysis of Physical Attractiveness in the Formation of First Impressions" conducted by Jewel Hunter. I understand that the purpose of this study is to learn more about interpersonal communication and first impressions.

I understand that my participation in the study involves completing a biographical questionnaire, a one-item rating scale for each picture and ranking sixteen photographs. I has been explained to me that my name and identifying personal informaion will be kept strictly confidential and that the information collected will be used for research and educational purposes only. The amount of time needed for my participation will be approximately one hour. Jewel Hunter will be present to give me the instructions to assess the sixteen individuals in separate photographs and answer any further questions I may have pertaining to the study or my participation.

I understand that upon completion of this study I will receive a copy of the abstract report of the research findings. It is further understood that I can withdraw from participation in this study at any time. I have read and understood the above information and I agree to participate in the study.

DATE : NAME (Printed):

SIGNATURE : 


\section{CONSENT FORM: QUESTIONNAIRE JUDGES}

I hearby give authorization and grant permission for participating as a questionnaire judge in the study, "A Multidimensional Analysis of Physical Attractiveness in the Formation of First Impressions" conducted by Jewel Hunter. I understand that the purpose of this study is to learn more about interpersonal communication and first impressions.

This study involves me completing two questionnaires. The first one is a brief biographical sketch and the second one is a questionnaire on my first impressions of an individual in a series of photographs. It has been explained to me that my name and identifying personal information will be kept strictly confidential and that the information collected will be used for research and educational purposes only. The amount of time needed for my participation will be approximately one hour. Jewel Hunter will be present to give me the instructions to assess the individual in separate photographs and answer any further questions I may have pertaining to the study or my participation.

I understand that upon completion of this study I will receive a copy of the abstract report of the research findings. It is further understood that I can withdraw from participation in this study at any time. I have read and understood the above information and I agree to participate in the study.

DATE : NAME (Printed): 


\section{CONSENT FORM: SUBJECTS}

I hearby give authorization and grant permission to serve as a subject in the study, "A Multidimensional Analysis of Physical Attractiveness in the Formation of First Impressions" conducted by Jewel Hunter. I understand that the purpose of this study is to learn more about interpersonal communication and first impressions.

This study involves me completing two questionnaires. The first one is a brief biographical sketch and the second one is a questionnaire on my first impressions of an individual in a series of photographs. It has been explained to me that my name and identifying personal information will be kept strictly confidential and that the information collected will be used for research and educational purposes only. The amount of time needed for my participation will be approximately one hour. Jewel Hunter will be present to give me the instructions to assess the individual in separate photographs and answer any further questions I may have pertaining to the study or my participation.

I understand that upon completion of this study I will receive a copy of the abstract report of the research findings. It is further understood that I can withdraw from participation in this study at any time. I have read and understood the above information and I agree to participate in the study.

DATE : NAME (Printed):

SIGNATURE : 
Jewel Hunter

Speech Communication/Gerontology

Institute on Aging

Portl and State University

P.0. Box 751

Portland, OR 97207

(503) 229-3952

A Multidimensional Analysis of Physical Attractiveness in the Formation of First Impression

A Note on Anonymity

A vital concern of the investigator is the importance of anonymity in research. As a participant in this study, you will be assigned an identification number for both your biographical sketch and a questionnaire on your first impression of an individual in a series of photographs. The number assigned to you is to help identify both the demographic information and the interpersonal questionnaire as belonging together in a set. Only you and the researcher will know what your identification number is. Your questionnaires will not be identified at anytime by your name. 
APPENDIX $C$

THANK YOU LETTERS 
THANK YOU LETTER: STIMULI

Re: "A Multidimensional Analysis of Physical Attractiveness in the Formation of First Impressions"

Thank you very much for taking the time to come have your photograph taken and for completing the questionnaire. Your participation was an extremely important contribution to make my study, "A Multidimensional Analysis of Physical Attractiveness in the Formation of First

Impressions". This project could not have taken place without your assistance.

As promised, you will find enclosed with this letter a $5 \times 7$, color photograph of youself. Upon completion of this study, you will receive a copy of the abstract report of my research findings. If you have any questions or would like to receive a more detailed copy of the report, please contact me or leave a message at my work phone number, (292-3952).

In the future, I may be undertaking further research projects related to social psychology and gerontology. If you would be willing to participate in another study, please contact me.

Thank you again for your participation.

Yours sincerely,

Jewel Hunter

Enclosure 
THANK YOU LETTER: PHOTO AND SELECTION JUDGES

Re: "A Multidimensional Analysis of Physical Attractiveness in the Formation of First Impressions"

Thank you very much for taking the time to participate as a judge in this study, "A Multidimensional Analysis of Physical Attractiveness in the Formation of First Impressions". This project could not have taken $\mathrm{place}$ without your assistance.

Upon completion of this study, you will receive a copy of the abstract report of my research findings. If you have any questions or would like to receive a more detailed copy of the report, please contact me or leave a message at my work phone number, (292-3952).

In the future, I may be undertaking further research projects related to social psychology and gerontology. If you would be willing to participate in another study, please contact me.

Thank you again for your participation.

Yours sincerely,

Jewel Hunter 
THANK YOU LETTER: QUESTIONNAIRE JUDGES

Re: "A Multidimensional Analysis of Physical Attractiveness in the Formation of First Impressions"

Thank you very much for taking the time to participate as a questionnaire judge in this study, "A Multidimensional Analysis of Physical Attractiveness in the Formation of First Impressions". This project could not have taken place wi thout your assistance.

Upon completion of this study, you will receive a copy of the abstract report of my research findings. If you have any questions or would like to receive a more detailed copy of the report, please contact me or leave a message at my work phone number, (292-3952).

In the future, I may be undertaking further research projects related to social psychology and gerontology. If you would be willing to participate in another study, please contact me.

Thank you again for your participation.

Yours sincerely,

Jewel Hunter 
THANK YOU LETTER: SUBJECTS Re: "A Multidimensional Analysis of Physical Attractiveness in the
Formation of First Impressions"

Thank you very much for taking the time to participate as a subject in this study, "A Multidimensional Analysis of Physical Attractiveness in the Formation of First Impressions". This project could not have taken place without your assistance.

Upon completion of this study, you will receive a copy of the abstract report of my research findings. If you have any questions or would like to receive a more detailed copy of the report, please contact me or leave a message at my work phone number, (292-3952).

In the future, I may be undertaking further research projects related to social psychology and gerontology. If you would be willing to participate in another study, please contact me.

Thank you again for your participation.

Yours sincerely,

Jewel Hunter 
APPENDIX D

QUESTIONNAIRES 
Q-1. Your Sex. (Circle number of your answer)

$$
\begin{aligned}
& \text { 1. MALE } \\
& \text { 2. FEMALE }
\end{aligned}
$$

Q-2. Your present age: YEARS.

Q-3. Are you presently: (Circle number)

$$
\begin{aligned}
& \text { 1. EMPLOYED FULL TIME } \\
& \text { 2. EMPLOYED PART TIME } \\
& \text { 3. UNEMPLOYED } \\
& \text { 4. RETIRED }
\end{aligned}
$$

Q-4. Please desribe your occupation:

TITLE:

KIND OF WORK DONE:

Circle number of your occupational classification:

1. MANUAL WORKERS/ODD JOBS/JANITORS/HEAVY LABOUR

2. PROTECTIVE SERVICE WORKERS/SEMI-SKILLED WORKERS

3. CLERKS/FIREPERSON/PRACTICAL NURSES/POLICEPERSON/AUTO MECHANICS/BARTENDERS/BUS DRIVERS

4. STUDENT/RETIRED/HOUSE-PERSON

5. SECRETARIAL-RECEPTIONIST/ELECTRICIANS/BOOKKEEPERS/SALESCLERK/ CARPENTER/BUTCHER

6. SOCIAL WORKERS/HIGH OR GRADE SCHOOL TEACHERS/SALESPERSON/ CONTRACTORS/OPTOMETRISTS

7. COLLEGE TEACHERS/TRAINED NURSES/CHIROPRACTOR/UNDERTAKERS/ LIBRARIANS/NEW SPAPER EDITORS/ASSISTANT MANAGERS/ACCOUNTANTS

8. PRESIDENT OF COLLEGE/DOCTORS/DENTISTS/EXECUTIVE ENGINEERS/ JUDGES/SUPERINTENDENTS/CHEMISTS/ARCHITECTS/REGIONAL \& DIVISIONAL MANAGER/CPA'S

If married, please describe your spouse occupation:

TITLE:

KIND OF WORK DONE: 
Circle number of your spouse's occupational classification:

1. MANUAL WORKERS/ODD JOBS/JANITORS/HEAVY LABOUR

2. PROTECTIVE SERVICE WORKERS/SEMI-SKILLED WORKERS

3. CLERKS/F IREPERSON/PRACTICAL NURSES/POLICEPERSON/AUTO MECHANICS/BARTENDERS/BUS DRIVERS

4. STUDENT/RETIRED/HOUSE-PERSON

5. SECRETARIAL-RECEPTIONIST/ELECTRICIANS/BOOKKEEPERS/SALESCLERK/ CARPENTER/BUTCHER

6. SOCIAL WORKERS/HIGH OR GRADE SCHOOL TEACHERS/SALESPERSON/ CONTRACTORS/OPTOMETRISTS

7. COLLEGE TEACHERS/TRAINED NURSES/CHIROPRACTOR/UNDERTAKERS/ LIBRARIANS/NEWSPAPER EDITORS/ASSISTANT MANAGERS/ACCOUNTANTS

8. PRESIDENT OF COLLEGE/DOCTORS/DENTISTS/EXECUTIVE ENGINEERS/ JUDGES/SUPERINTENDENTS/CHEMISTS/ARCHITECTS/REGIONAL \& DIVISIONAL MANAGER/CPA's

Q-5. What was your approximate gross family income from all sources, before taxes, in 1981? (Circle number)

1. LESS THAN $\$ 4,999$

2. 5,000 to 8,999

3. 9,000 to 12,999

4. 13,000 to 16,999

5. 17,000 to 20,999

6. 21,000 to 24,999

7. 25,000 to 28,999

8. OVER $\$ 29,000$

Q-6. Which is the highest level of education that you have completed? (Circle number)

1. NO FORMAL EDUCATION

2. SOME GRADE SCHOOL

3. COMPLETED GRADE SCHOOL

4. SOME HIGH SCHOOL

5. COMPLETED HIGH SCHOOL

6. SOME COLLEGE

7. COMPLETED COLLEGE (Specify Major)

8. SOME GRADUATE WORK

9. A GRADUATE DEGREE

(Specify Degree and Major)

Q-7. Are you presently: (Circle number)

1. NOT A COLLEGE/UNIVERSITY STUDENT

2. ENROLLED PART TIME AS A COLLEGE/UNIVERSITY STUDENT

3. ENROLLED FULL TIME AS A COLLEGE/UNIVERSITY STUDENT

4. NOT ENROLLED AS A COLLEGE/UNIVERSITY STUDENT, BUT WORKING ON THESIS/DISSERTATION. 
Q-8. Check the appropriate box below indicating your sexuality:

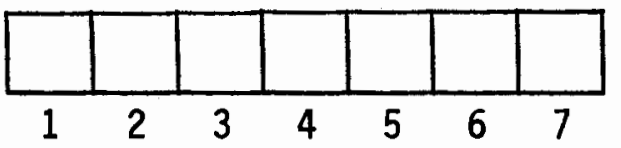

Completely Completely

Homosexual Heterosexual

Q-9. What is your street address?

(Street)

(City) (State)

(Zip)

Phone Number: (Home)

(Work) 
INSTRUCTIONS TO SELECTION JUDGES USING THE SEVEN POINT RATING SCALE TO EVALUATE STIMULUS PHOTOGRAPHS PHYSICAL ATTRACTIVENESS

This study is designed to investigate First Impressions of people. You will be shown a series of photographs consisting of 16 people. There is one statement followed by a seven point scale labeled at the end points, Very Physically Attractive (1) to Very Physically Unattractive (7). You are to answer by checking one of the seven answer choices. As a preliminary precaution, please indicate if you know or have seen this person in the photograph before by checking the appropriate box. After you have responded to the first photograph, you are to turn the photo and slip of paper consisting of the scale over and continue to the next one until all 16 stimulus photographs have been evaluated. Do not be concerned about the amount of time it will take to evaluate them.

Your honest and frank opinion is very important in helping to analyze the results of this study. This is not a test of you personally, nor will any of these answers be identified as belonging to particular individuals. Your answers will be kept strictly confidential.

If you have any questions, please feel free to stop at any time to ask them. This will not effect the results of the study. 
Questionnaires: Selection Judges Seven Point Rating Form to Evaluate Stimulus Photographs Physical Attractiveness.

PHOTO IO

JUDGE ID

IN YOUR OPINION: HOW PHYSICALLY ATTRACTIVE IS THIS PERSON TO YOU? (PTease check the appropriate box below)

VERY PHYSICALLY
ATTRACTIVE

ATTRACTIVE YOU KNOH

DO YOU KNOW OR HAVE YOU SEEN THIS PERSON IN THE PHOTOGRAPH BEFORE?

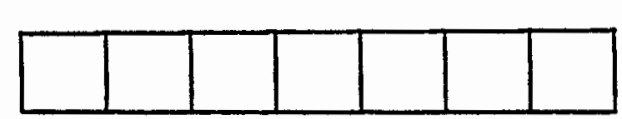

VERY PHYSICALLY UNATTRACTIVE

$\square$ YES $\square$ NO 
SELECTION JUDGE ID

INVESTIGATOR'S OBSERVATION SHEET OF JUDGES

(1) Rated Photos

(2) Ranked Photos

TASK: Rank 16 photographs of who is most physically attractive to least physically attractive spreading them out on the table. Females first and then males. Now pick out the most physically attractive female/male and unattractive and then the most physically unattractive female/male.

MALE STIMULUS PHOTOGRAPHS RANKING ORDER:

VERY PHYSICALLY

ATTRACTIVE

VERY PHYSICALLY UNATTRACTIVE

FEMALE STIMULUS PHOTOGRAPHS RANKING ORDER:

$\begin{array}{ll}\text { VERY PHYSICALLY } & \text { VERY PHYSICALLY } \\ \text { ATTRACTIVE } & \text { UNATTRACTIVE }\end{array}$

OBSERVATIONS: (OF JUDGE'S BEHAVIOR IN THE PROCESS OF RANKING THE PHOTOGRAPHS)

(3) Tape Recorded: What is about that person that you find very physically attractive?

What is about that person that you find least physically attractive?

I know this is really difficult but it is really important that you tell me what it is. 


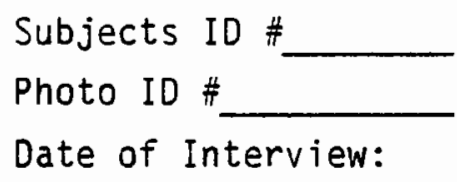

Original First Impression Questionnaire (OFIQ)

\author{
Portland State University \\ Speech Communication \\ Gerontology
}

Do you or have you seen this person in the photograph before?

YES

NO

C1986 Jewe1 M. Smith Hunter. OFIQ was used in "A Multidimensional Analysis of Physical Attractiveness in the Formation of First Impressions," Master of Science Thesis at Portland State University. 


\section{INTERPERSONAL COMMUNICATION STUDY}

This questionnaire on interpersonal communication is designed to investigate first impression of people. You will be shown photographs of eighteen persons. You are to indicate your first impression of these persons with respect to 30 traits and interpersonal characteristics. "Traits" of photographed persons are listed above each positive-negative scale. You are to answer by checking one of the seven answer choices. Be sure and rate all of the traits on the inventory. Do not be concerned about the amount of time it will take to evaluate them.

Your honest and frank opinion is very important in helping to analyze the results of this study. This is not a test of you personally, nor will any of these answers be identified as belonging to particular individuals. Your answers will be kept strictly confidential.

If you have any questions, please feel free to stop at any time to ask them. This will not affect the results of the study. 


\section{INSTRUCTIONS}

This is a pretest to help the researcher construct the main questionnaire to be used in the final study. Your opinion is vital and very important in developing this instrument. Please write down any comments beside any item that may appear unclear to you.

FOR EACH ITEM, PLEASE ANSWER BY CHECKING ONE OF THE 7 ANSWER CHOICES

EX.-01 THIS PERSON'S EYELASHES ARE:

VERY PHYSICALLY

ATTRACTIVE

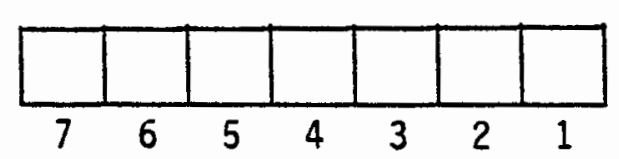

VERY PHYSICALLY

UNATTRACTIVE

EX.-02 I THINK THIS PERSON IS A DULL PERSON:

STRONGLY

AGREE

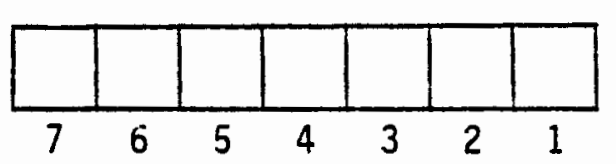

STRONGLY

DISAGREE

EX.-03 THIS PERSON APPEARS:

DECISIVE

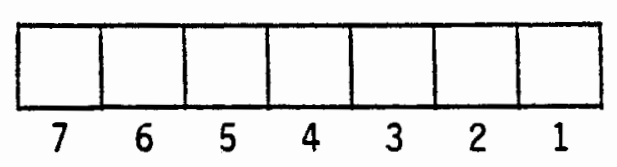

INDECISIVE 
I-1. THIS PERSON'S PHYSICAL APPEARANCE IS:

VERY PHYSICALLY

ATTRACT IVE

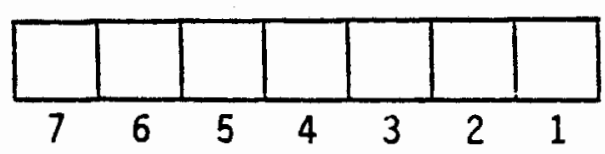

VERY PHYSICALLY UNATTRACTIVE

I-2.

THIS PERSON'S NOSE IS:

VERY PHYSICALLY ATTRACTIVE

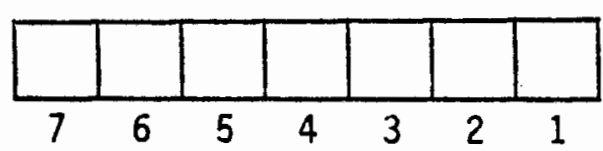

I-3. THIS PERSON'S FACE SHAPE IS:

VERY PHYSICALLY ATTRACTIVE

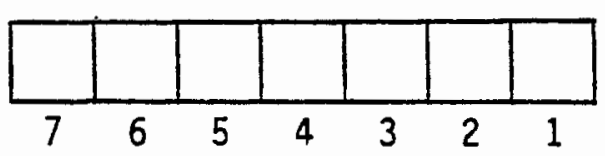

I-4. THIS PERSON'S EYEBROWS ARE:

VERY PHYSICALLY ATTRACTIVE

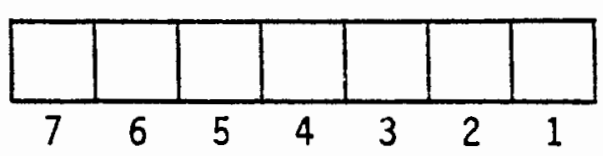

I-5. THIS PERSON'S FACIAL GROOMING IS:

VERY PHYSICALLY ATTRACTIVE

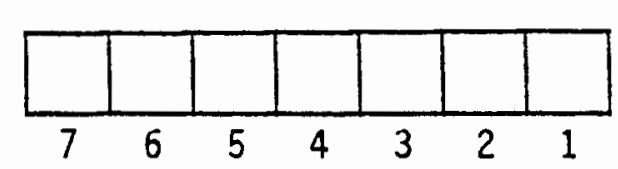

VERY PHYSICALLY UNATTRACTIVE

VERY PHYSICALLY UNATTRACTIVE

VERY PHYSICALLY UNATTRACTIVE

VERY PHYSICALLY UNATTRACTIVE 
I-6. THIS PERSON'S IS SMILE IS:

VERY PHYSICALLY

ATTRACTIVE

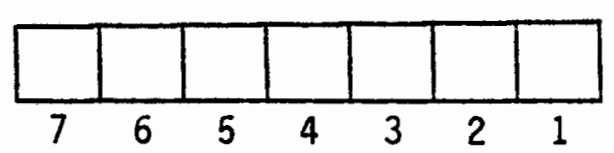

VERY PHYSICALLY

UNATTRACTIVE

I-7. THIS PERSON'S WEIGHT IS:

VERY PHYSICALLY ATTRACTIVE

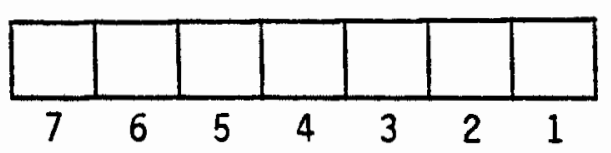

I-8. THIS PERSON'S TEETH ARE:

VERY PHYSICALLY ATTRACTIVE

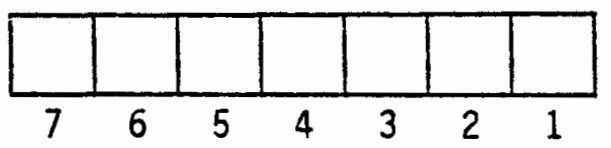

I-9. THIS PERSON'S HAIRSTYLE IS:

VERY PHYSICALLY ATTRACTIVE

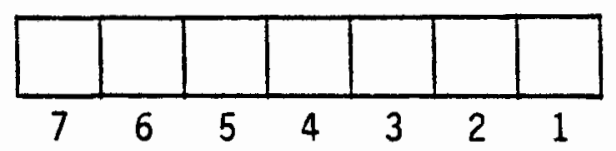

VERY PHYSICALLY UNATTRACTIVE

I-10. THIS PERSON'S PERSOMAL HYGIENE IS:

VERY PHYSICALLY ATTRACTIVE
VERY PHYSICALLY

UNATTRACTIVE
VERY PHYSICALLY UNATTRACTIVE
VERY PHYSICALLY UNATTRACTIVE

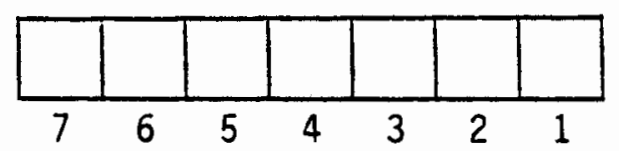


I-11. THIS PERSON'S CHIN IS:

VERY PHYSICALLY ATTRACTIVE

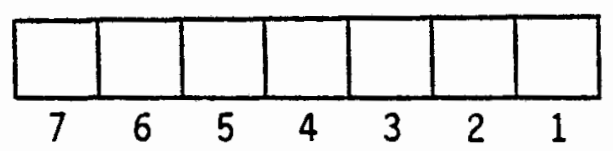

I-12. THIS PERSON'S EYES ARE:

VERY PHYSICALLY ATTRACTIVE

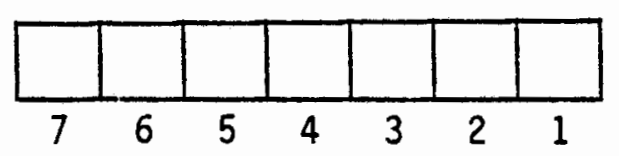

I-13. THIS PERSON'S POSTURE IS:

VERY PHYSICALLY ATTRACTIVE

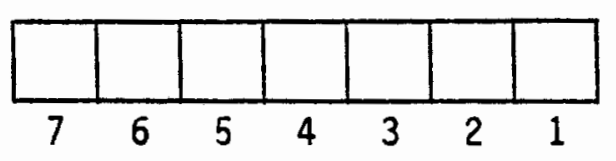

I-14. THIS PERSON'S MOUTH IS:

VERY PHYSICALLY ATTRACTIVE

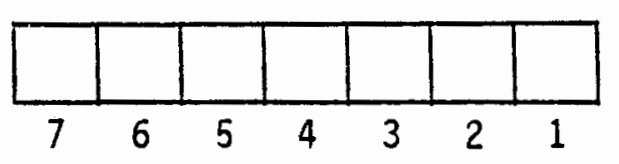

I-15. THIS PERSON'S FACIAL EXPRESSION IS:

VERY PHYSICALLY ATTRACT IVE

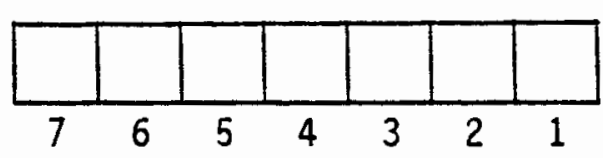

VERY PHYSICALLY UNATTRACTIVE

VERY PHYSICALLY UNATTRACTIVE

VERY PHYSICALLY UNATTRACTIVE

VERY PHYSICALLY UNATTRACTIVE

VERY PHYSICALLY UNATTRACT IVE 
I-16. THIS PERSON'S SKIN IS:

VERY PHYSICALLY ATTRACTIVE

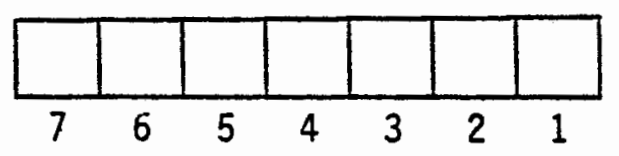

I-17. THIS PERSON'S EARS ARE:

VERY PHYSICALLY ATTRACTIVE

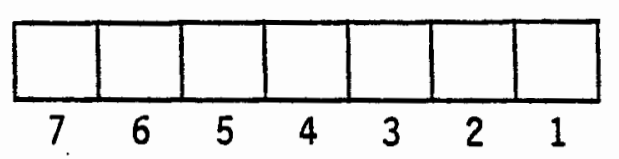

I-18. THIS PERSON'S HEALTH IS:

VERY PHYSICALLY ATTRACTIVE

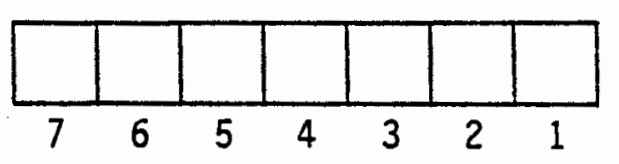

I-19. THIS PERSON'S NECK IS:

VERY PHYSICALLY ATTRACTIVE

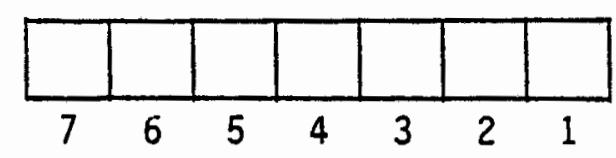

I-20. THIS PERSON'S HAIR IS:

VERY PHYSICALLY ATTRACT IVE

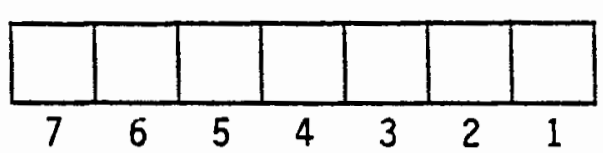

VERY PHYSICALLY UNATTRACTIVE

VERY PHYSICALLY UNATTRACTIVE

VERY PHYSICALLY UNATTRACTIVE

VERY PHYSICALLY UNATTRACTIVE

VERY PHYSICALLY UNATTRACT IVE 
I-21. THIS PERSON IS WELL GROOMED:

STRONGLY AGREE

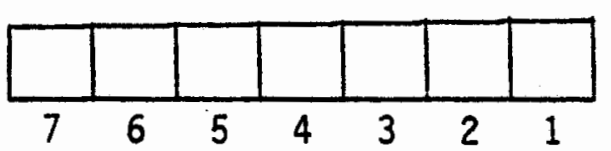

I-22. THIS PERSON IS:

VERY PHYSICALLY ATTRACTIVE

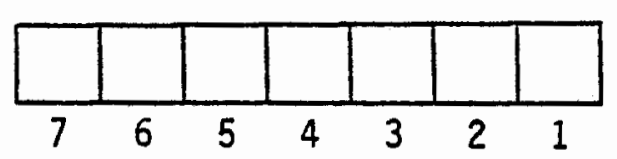

STRONGLY DISAGREE

VERY PHYSICALLY

UNATTRACTIVE

SECTION II.

I would like you to answer how you feel on the following 14 items consisting of descriptive traits (ranging from opposite to opposite) about the person in the photograph.

I-23. THIS PERSON APPEARS:

INDEPENDENT

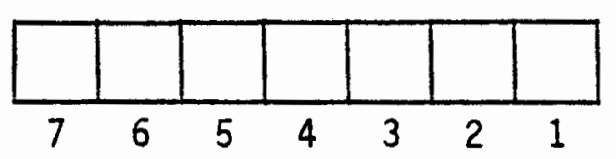

I-24. THIS PERSON APPEARS:

PLEASANT

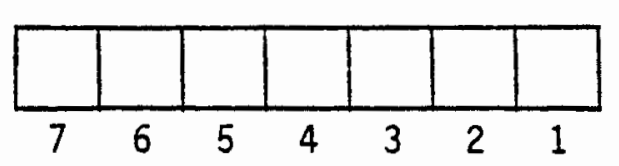

DEPENDENT

\section{UNPLEASANT}


I-25. THIS PERSON APPEARS:

ACTIVE

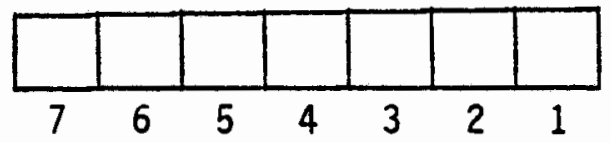

PASSIVE

I-26. THIS PERSON APPEARS:

OPEN MINDED

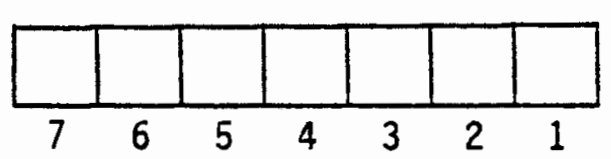

CLOSED MINDED

I-27. THIS PERSON APPEARS:

ENTHUSIASTIC

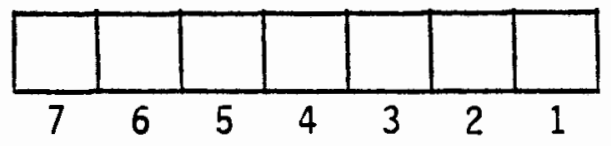

UNENTHUSIASTIC

I-28. THIS PERSON APPEARS:

CONSIDERATE

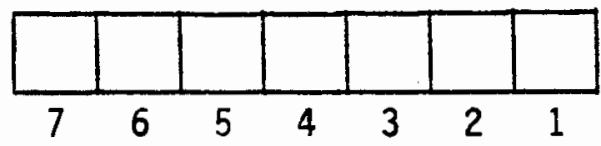

INCONS IDERATE

I-29. THIS PERSON APPEARS:

FAST

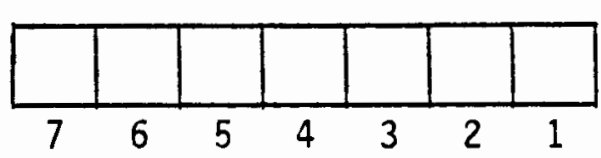

SLOW

I-30. THIS PERSON APPEARS:

LOVING

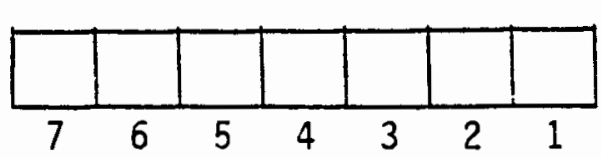

UNLOVING 
I-31. THIS PERSON APPEARS:

STRONG

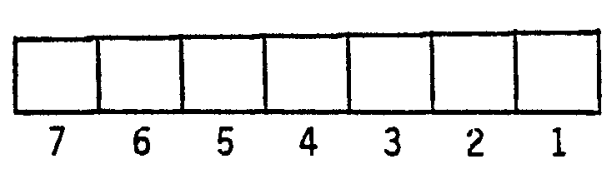

WEAK

I-32. THIS PERSON APPEARS:

COOPERATIVE

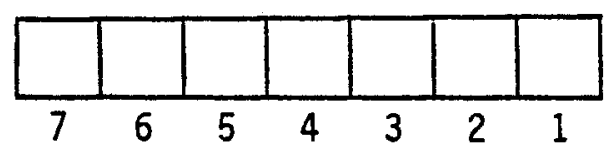

UNCOOPERATIVE

I-33. THIS PERSON APPEARS:

AMBITIOUS

HARDW ORKING

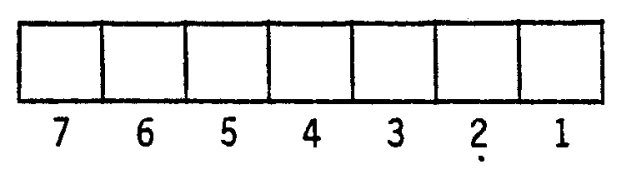

UNAMB ITIOUS,

LAZY

I-34. THIS PERSON APPEARS:

UNDERSTANDING

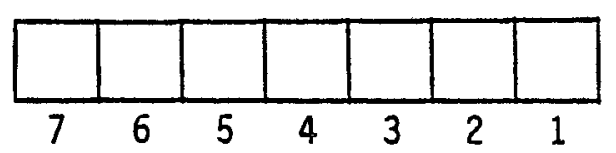

NOT UNDERSTANDING

I-35. THIS PERSON APPEARS:

ALERT

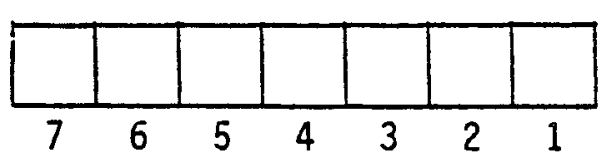

NONALERT 
I-36. THIS PERSON APPEARS:

FLEXIBLE

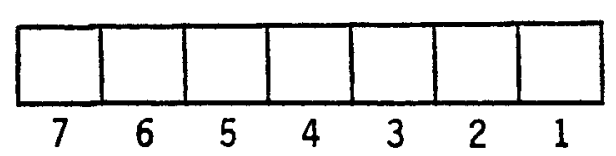

INFLEXIBLE

SECTION III.

Another important part of this study is to learn more about interpersonal behavior. I would like you to indicate your degree of agreement with each of the following statements by checking how you feel.

I-37. I BELIEVE I WOULD ENJOY HAVING A FRIENDLY CHAT WITH THIS PERSON.

STRONGLY AGREE

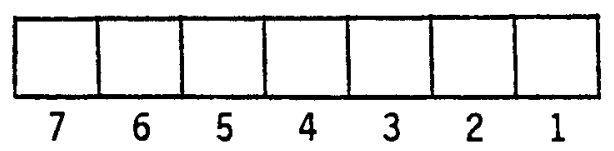

STRONGLY DISAGREE

I-38. IF I WERE SPEAKING TO THIS PERSON, I WOULD TALK MORE FREQUENTLY THAN USUAL.

STRONGLY AGREE

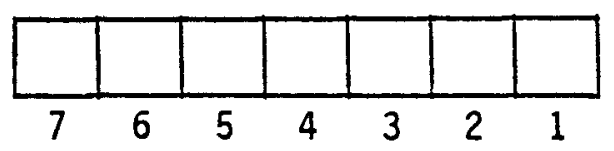

STRONGLY DISAGREE

I-39. I THINK I WOULD ENJOY KNOWING THIS PERSON.

STRONGLY AGREE

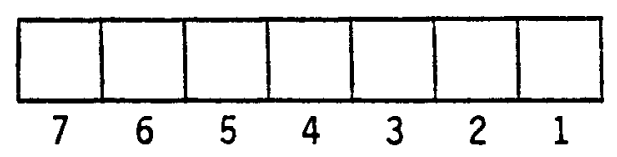

STRONGLY DISAGREE 
I-40. IF I WERE IN AN INFORMAL CONVERSATION WITH THIS PERSON, I THINK I WOULD BE INCLINED TO DOMINATE THE CONVERSATION.

STRONGLY AGREE

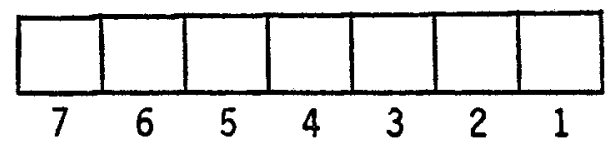

STRONGLY DISAGREE

I-41. IF I SPOKE TO THIS PERSON I WOULD PROBABLY PAUSE QUITE OFTEN IN THE COURSE OF THE CONVERSATION.

STRONGLY AGREE

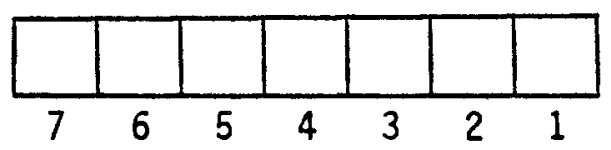

STRONGLY DISAGREE

I-42. I AM ATTRACTED TO THIS PERSON.

STRONGLY AGREE

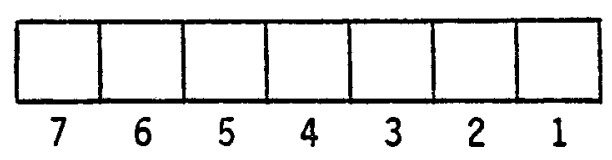

STRONGLY DISAGREE

I-43. IF I WERE HAVING A SMALL DINNER PARTY, I WOULD BE PLEASED TO INVITE THIS PERSON.

STRONGLY AGREE

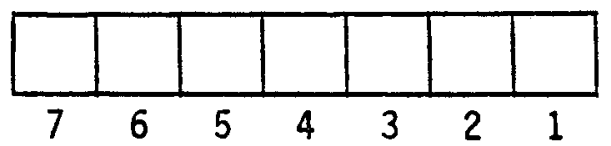

STRONGLY DISAGREE

I-44. I WOULD GLADLY RESPOND TO THIS PERSON IF HE/SHE INITIATED A CONVERSATION.

STRONGLY AGREE

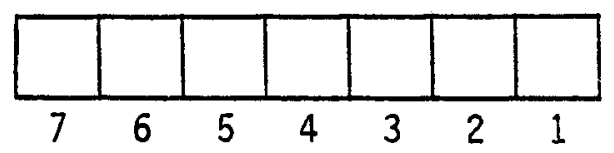

STRONGLY DISAGREE 
I-45. I THINK I WOULD ADMIRE THIS PERSON.

STRONGLY AGREE

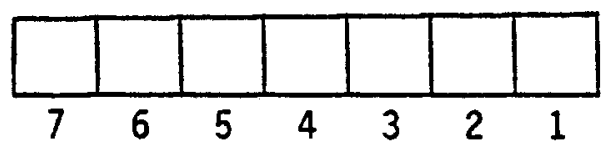

STRONGLY DISAGREE

I-46. IF I SAW THIS PERSON, I WOULD PROBABLY ACKNONLEDGE HIM/HER WHEN PASSING.

STRONGLY AGREE

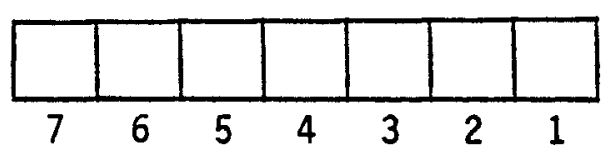

STRONGLY DISAGREE

I-47. I THINK I WOULD ENJOY SPENDING A WEEK'S VACATION WITH THIS PERSON.

STRONGLY AGREE

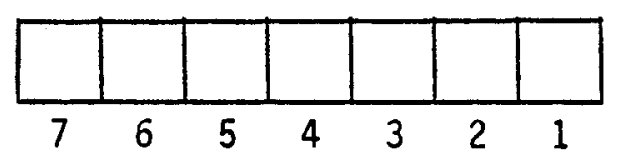

STRONGLY DISAGREE

I-48. I THINK I COULD FEEL CLOSE TO THIS PERSON.

STRONGLY AGREE

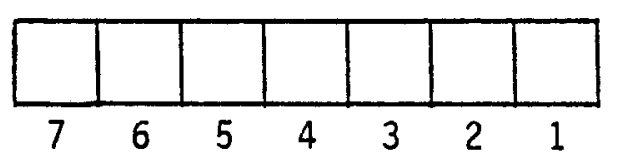

STRONGLY DISAGREE

I-49. IF I WAS IN A SOCIAL CONVERSATION WITH THIS PERSON, I WOULD NOT BE INCLINED TO LET HIM/HER GET IN THE LAST WORD.

STRONGLY AGREE

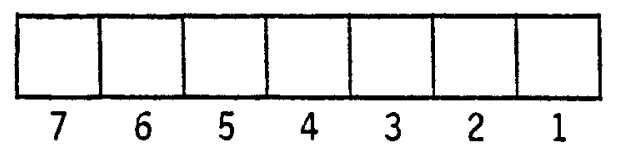

STRONGLY DISAGREE 
I-50. I WOULD CALL THIS PERSON BY HIS/HER FIRST NAME.

STRONGLY AGREE

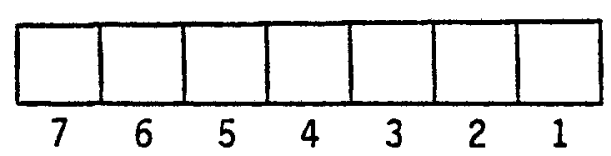

STRONGLY DISAGREE

I-51. I FEEL FAVORABLE TONARDS KNONING THIS PERSON.

STRONGLY AGREE

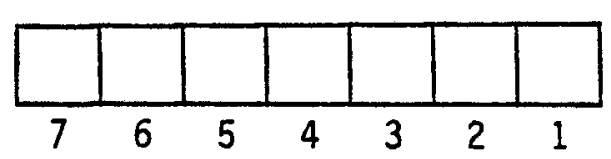

STRONGLY DISAGREE

I-52. IF I WERE HAVING A ONE-TO-ONE CONVERSATION WITH THIS PERSON, I WOULD TEND TO LET HIM/HER TALK MORE THAN HALF THE TIME.

STRONGLY AGREE

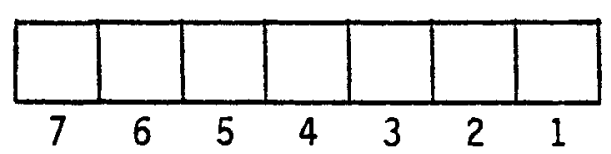

STRONGLY DISAGREE

I-53. I WOULD TEND TO HESITATE BEFORE SPEAKING TO THIS PERSON.

STRONGLY AGREE

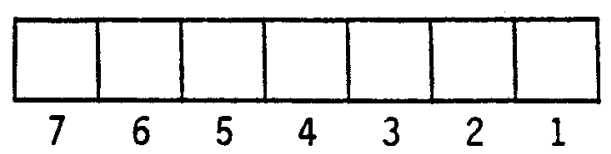

STRONGLY DISAGREE

I-54. THIS PERSON APPEALS TO ME.

STRONGLY AGREE

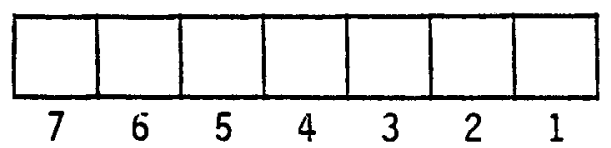

STRONGLY DISAGREE 
I-55. I THINK I WOULD ENJOY INVITING THIS PERSON TO MY HOME FOR DINNER.

STRONGLY AGREE

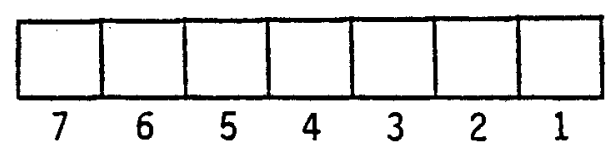

STRONGLY DISAGREE

I-56. IN SPEAKING TO THIS PERSON, I WOULD TEND TO TALK FOR LONG PERIODS OF TIME.

STRONGLY AGREE

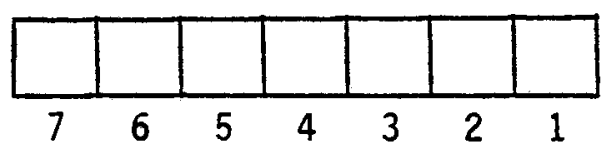

STRONGLY DISAGREE

I-57. I THINK I WOULD FEEL AMIABLE TOWARDS THIS PERSON.

STRONGLY AGREE

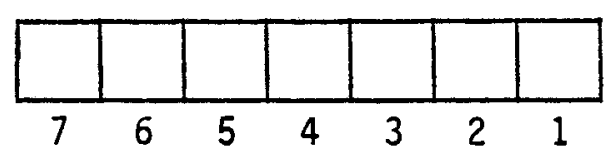

STRONGLY DISAGREE

I-58. IF I WERE TO SPEAK WITH THIS PERSON, I WOULD BE INCLINED TO LET HIM/HER START THE CONVERSATION.

STRONGLY AGREE

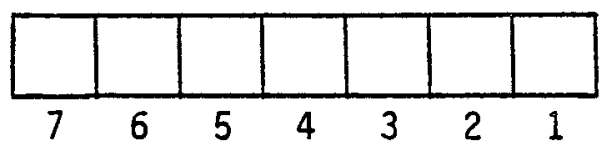

STRONGLY DISAGREE

I-59. I THINK I WOULD ENJOY SPENDING AN AFTERNOON WITH THIS PERSON.

STRONGLY AGREE

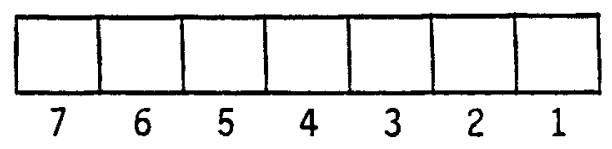

STRONGLY DISAGREE 
I-60. I AM INTERESTED IN KNOWING THIS PERSON.

ȘTRONGLY AGREE

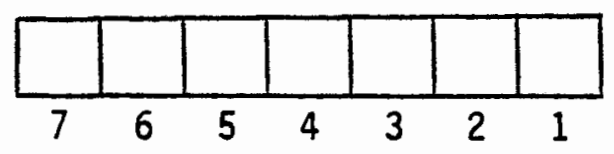

STRONGLY DISAGREE

I-61. IF I WERE WITH THIS PERSON, I WOULD RELY ON HIM/HER TO KEEP THE CONVERSATION GOING.

STRONGLY AGREE

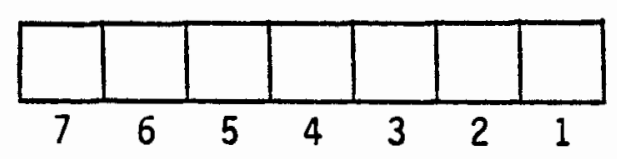

STRONGLY DISAGREE

1-62. IF I WERE WITH THIS PERSON, IT WOULD PROBABLY TAKE ME QUITE A WHILE TO WARM UP AND SAY VERY MUCH.

STRONGLY AGREE

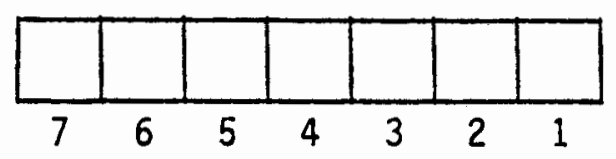

STRONGLY DISAGREE

I-63. I FEEL I CAN IDENTIFY WITH THIS PERSON.

STRONGLY AGREE

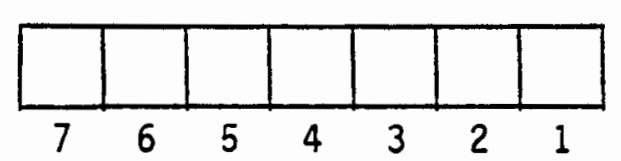

STRONGLY DISAGREE

I-64. MOST PEOPLE WOULD REACT FAVORABLY TO THIS PERSON.

STRONGLY AGREE

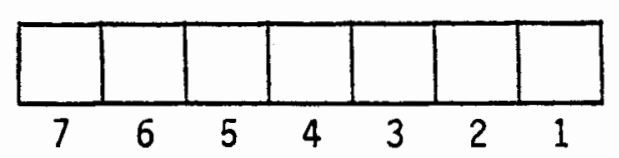

STRONGLY DISAGREE 
I-65. I WOULD PREFER TO SPEAK TO THIS PERSON RATHER THAN TO LISTEN TO HIM/HER.

STRONGLY AGREE

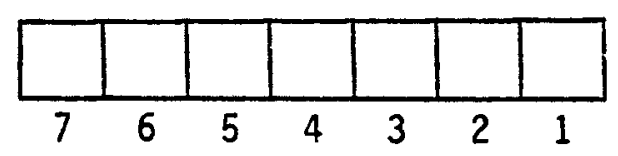

STRONGLY DISAGREE

I-66. I WOULD FEEL WARMLY TONARDS THIS PERSON.

STRONGLY AGREE

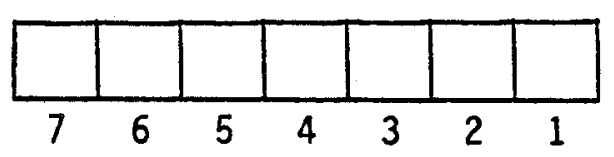

STRONGLY DISAGREE

I-67. I WOULD CONSIDER HAVING THIS PERSON AS A CLOSE, INTIMATE FRIEND.

STRONGLY AGREE

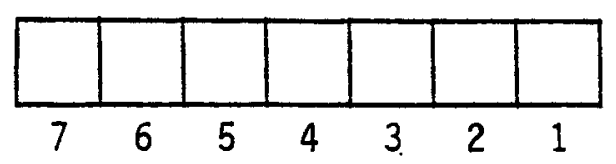

STRONGLY DISAGREE

I-68. IF I WERE WITH THIS PERSON IN A SOCIAL CONVERSATION, I WOULD DIRECT THE COURSE OF IT.

STRONGLY AGREE

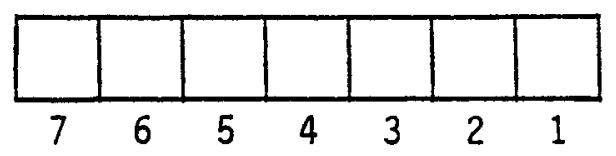

I-69. I WISH I COULD MEET THIS PERSON.

STRONGLY AGREE

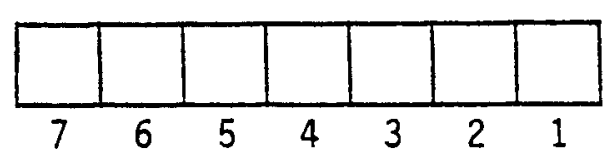

STRONGLY DISAGREE

STRONGLY DISAGREE 
I-70. ON A JOB, I BELIEVE I WOULD ENJOY HAVING THIS PERSON AS A COW ORKER.

STRONGLY AGREE

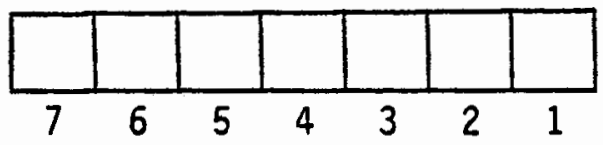

STRONGLY OISAGREE

I-71. IF I WERE HAVING A CONVERSATION WITH THIS PERSON, I WOULD PROBABLY SPEAK SHORTER PERIODS OF TIME THAN USUAL.

STRONGLY AGREE

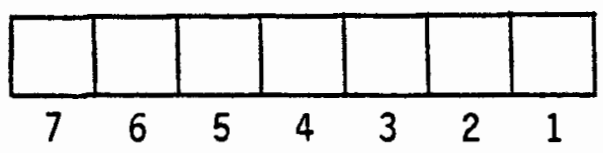

STRONGLY DISAGREE

I-72. I THINK THIS PERSON IS SIMILAR TO ME.

STRONGLY AGREE

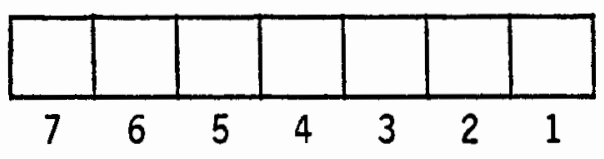

STRONGLY DISAGREE

I-73. IN SPEAKING WITH THIS PERSON, I FEEL I COULD EXPRESS MYSELF QUITE FREELY.

STRONGLY AGREE

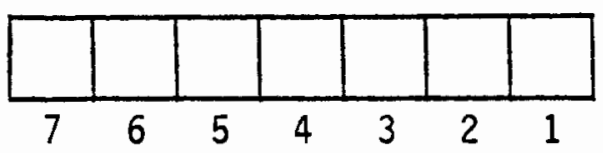

STRONGLY DISAGREE

I-74. IF SPEAKING TO THIS PERSON, I WOULD PROBABLY LET HIM/HER TALK FOR LONG PERIODS OF TIME.

STRONGLY AGREE

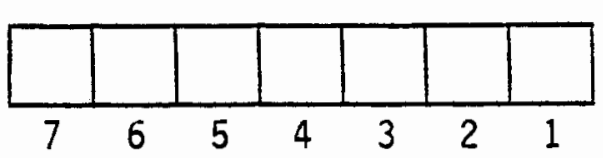

STRONGLY DISAGREE 
I-75. THIS PERSON IS REPULSIVE TO ME.

STRONGLY AGREE

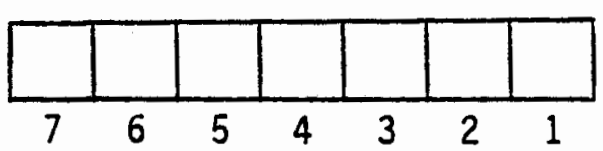

STRONGLY DISAGREE

I-76. IN CONVERSING WITH THIS PERSON, I WOULD HAVE THE TENDENCY TO LET HIM/HER DETERMINE THE COURSE OF THE CONVERSATION.

STRONGLY AGREE

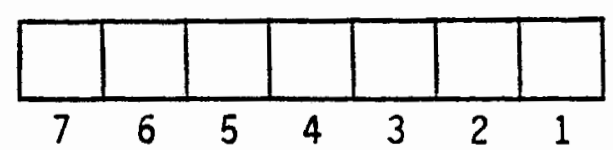

STRONGLY DISAGREE

I-77. IF I WERE WITH THIS PERSON, I WOULD PROBABLY INITIATE A CONVERSATION WITH HIM/HER.

STRONGLY AGREE

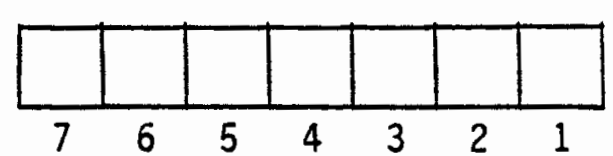

STRONGLY DISAGREE

I-78. I FEEL I COULD BECOME FRIENDS WITH THIS PERSON.

STRONGLY AGREE

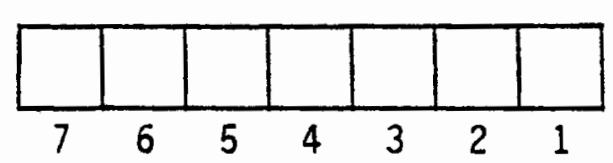

STRONGLY DISAGREE

I-79. IF I WERE IN A SOCIAL CONVERSATION WITH THIS PERSON, I WOULD PROBABLY COME ON STRONG.

STRONGLY AGREE

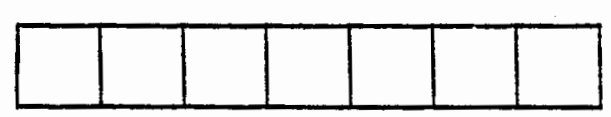

STRONGLY DISAGREF. 
I-80. IF I WERE ON A BUS, I WOULD PROBABLY SIT NEXT TO THIS PERSON.

STRONGLY AGREE

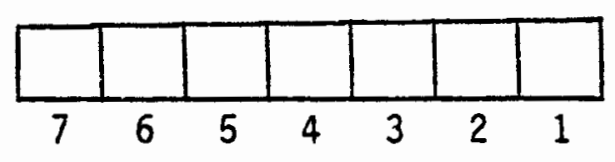

STRONGLY DISAGREE

I-81. I WOULD FEEL FOND OF THIS PERSON.

STRONGLY AGREE

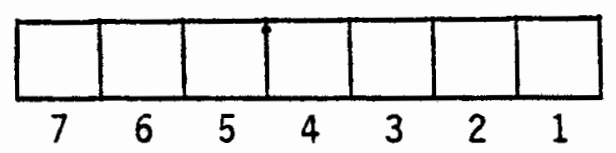

STRONGLY DISAGREE

I-82. IF I WERE IN A SOCIAL CONVERSATION WITH THIS PERSON, I WOULD SPEAK QUITE FREQUENTLY.

STRONGLY AGREE

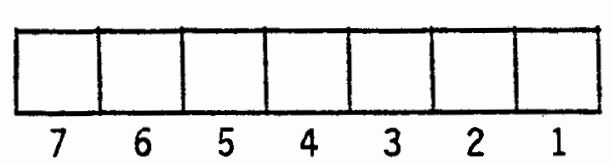

STRONGLY DISAGREE

I-83. IF I WERE SPEAKING TO THIS PERSON, I WOULD TRY TO TAKE CHARGE OF THINGS IN OUR CONVERSATION.

STRONGLY AGREE

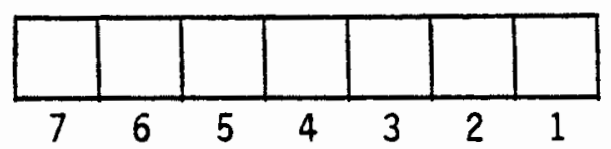

STRONGLY DISAGREE

I-84. I WOULD LOVE TO MEET THIS PERSON.

STRONGLY AGREE

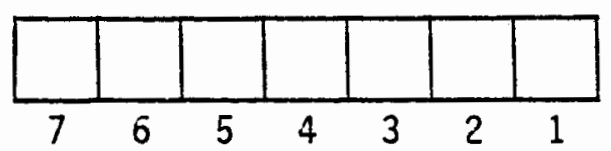

STRONGLY DISAGREE 
I-85. IF I WERE HAVING TROUBLE IN MY FAMILY, I PROBABLY WOULD CONFIDE IN THIS PERSON.

STRONGLY AGREE

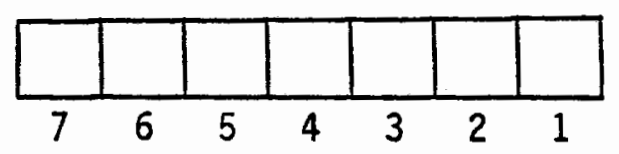

STRONGLY DISAGREE

I-86. IF I WERE HAVING A CONVERSATION WITH THIS PERSON, I WOULD PROBABLY BE INCLINED TO TALK MORE FORCEFUL THAN USUAL.

STRONGLY AGREE

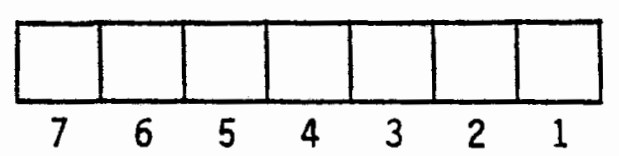

STRONGLY DISAGREE

I-87. I FEEL I WOULD DISLIKE KNOWING THIS PERSON.

STRONGLY AGREE

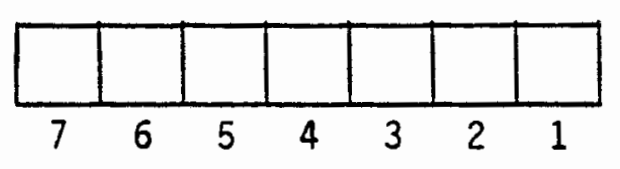

STRONGLY DISAGREE

I-88. IF I WERE IN A SOCIAL CONVERSATION WITH THIS PERSON, I WOULD PROBABLY BE INCLINED TO LET HIM/HER GET IN THE LAST WORD.

STRONGLY AGREE

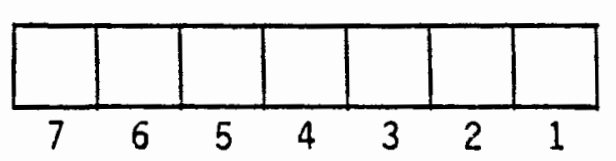

STRONGLY DISAGREE

I-89. IN SPEAKING TO THIS PERSON, I WOULD PROBABLY FEEL INHIBITED.

STRONGLY AGREE

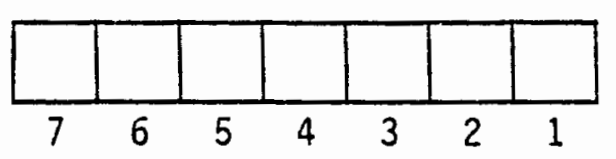

STRONGLY DISAGREE 
I-90. I FEEL I WOULD LIKE THIS PERSON.

STRONGLY AGREE

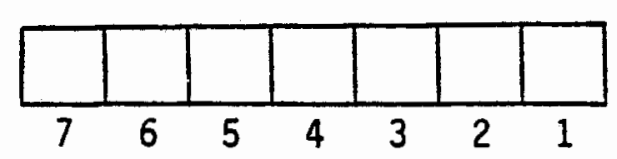

STRONGLY DISAGREE

I-91. IF I SPOKE TO THIS PERSON, I WOULD PREFER TO KEEP MY COMMENTS BRIEF.

STRONGLY AGREE

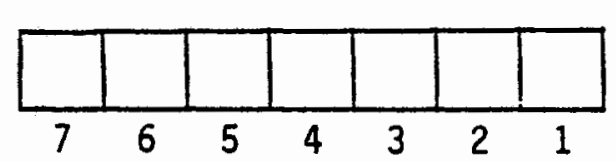

STRONGLY DISAGREE

I-92. I WOULD BE INCLINED TO JUMP INTO INFORMAL CONVERSATION WITH THIS PERSON.

STRONGLY AGREE

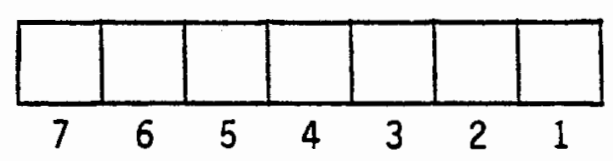

STRONGLY DISAGREE

I-93. IN SPEAKING WITH THIS PERSON, I WOULD FEEL I COULD NOT EXPRESS MY VIEWS.

STRONGLY AGREE

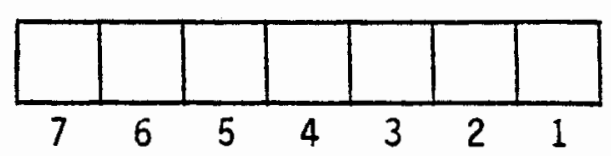

STRONGLY DISAGREE

I-94. IF I WERE IN A SOCIAL CONVERSATION WITH THIS PERSON, I WOULD PROBABLY DDMINATE IT.

STRONGLY AGREE

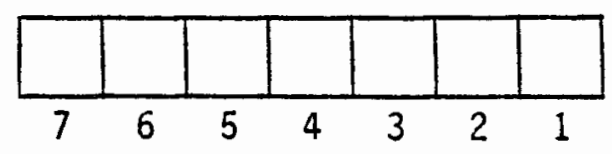

STRONGLY DISAGREE 
SECTION IV.

Finally, I would like you to respond to the last 11 items that deal with descriptive characteristics ranging from opposite to opposite about the person in the photograph.

I-95. THIS PERSON APPEARS:

KIND

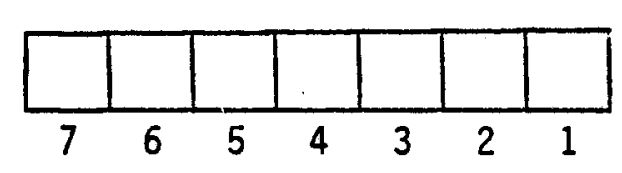

CRUEL

I-96. THIS PERSON APPEARS:

INTELLIGENT

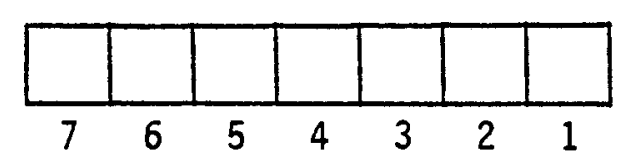

UNINTELLIGENT

I-97. THIS PERSON APPEARS:

MODEST

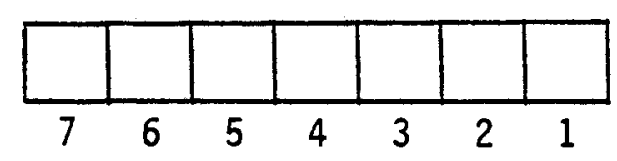

IMMODEST

I-98. THIS PERSON APPEARS:

POSITIVE

SELF-CONCEPT

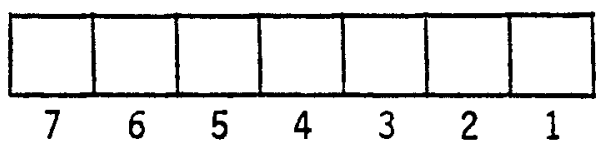

NEGATIVE

SELF-CONCEPT

I-99. THIS PERSON APPEARS:

SENS ITIVE

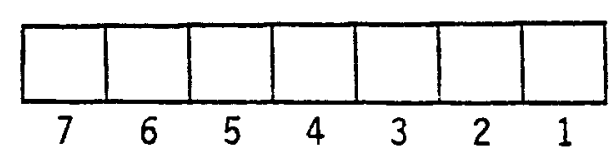

INSENSITIVE 
I-100. THIS PERSON APPEARS:

SINCERE

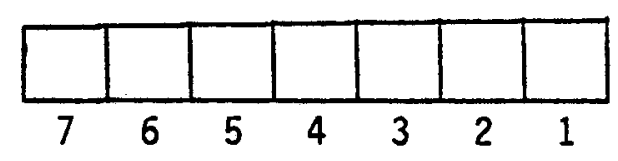

INS INCERE

I-101 THIS PERSON APPEARS:

FRIENDLY

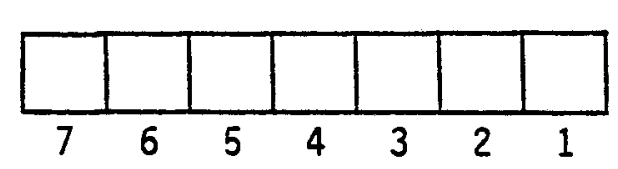

UNFRIENDLY

I-102. THIS PERSON APPEARS:

TIDY

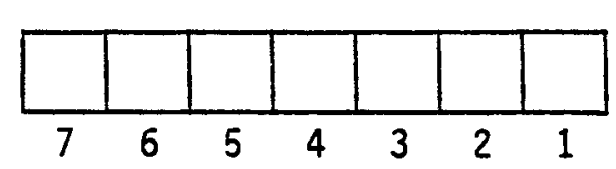

UNTIDY

I-103. THIS PERSON APPEARS:

LIKEABLE

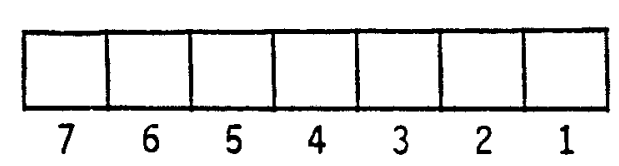

UNLIKEABLE

I-104. THIS PERSON APPEARS:

SOPHISTICATED

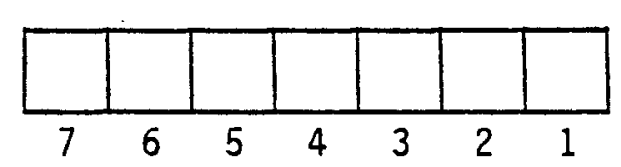

UNSOPHISTICATED

I-105. THIS PERSON APPEARS:

WARM

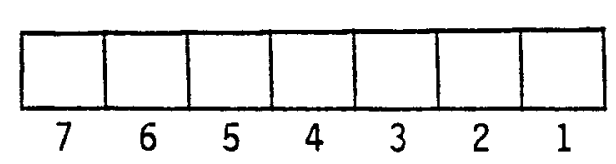

COLD 
Subjects ID $\#$

Photo ID \#

Date of Interview:

Modified First Impression Questionnaire (MFIQ)

\author{
Portland State University \\ Speech Communication \\ Gerontology
}

(91986 Jewe1 M. Smith Hunter. MFIQ was used in "A Multidimensional Analysis of Physical Attractiveness in the Formation of First Impressions," Master of Science Thesis at Portland State University. 
This questionnaire on interpersonal communication is designed to investigate first impression of people. You will be shown photographs of eighteen persons. You are to indicate your first impression of these persons with respect to 30 traits and interpersonal characteristics. "Traits" of photographed persons are listed above each positive-negative scale. You are to answer by checking one of the seven answer choices. Be sure and rate all of the traits on the inventory. Do not be concerned about the amount of time it will take to evaluate them.

Your honest and frank opinion is very important in helping to analyze the results of this study. This is not a test of you personaliy, nor will any of these answers be identified as belonging to particular individuals. Your answers will be kept strictly confidential.

If you have any questions, please feel free to stop at any time to ask them. This will not affect the results of the study. 


\section{INSTRUCTIONS}

FOR EACH ITEM, PLEASE ANSWER BY CHECKING ONE OF THE 7 ANSWER CHOICES

EX.-01 THIS PERSON'S EYELASHES ARE:

VERY PHYSICALLY ATTRACTIVE

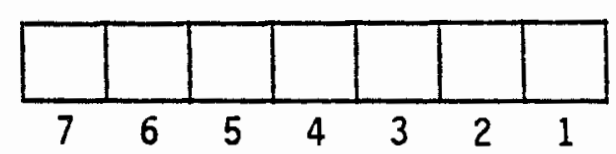

EX.-02 I THINK THIS PERSON IS A. DULL PERSON:

STRONGLY AGREE

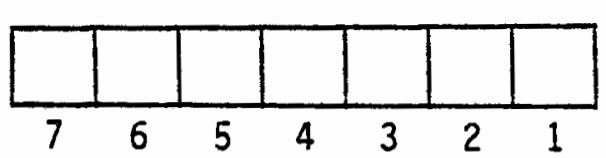

EX.-03 THIS PERSON APPEARS:

DECISIVE

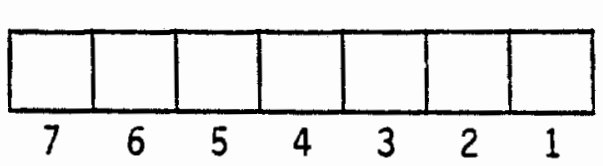

VERY PHYSICALLY UNATTRACT IVE

\section{STRONGLY}

DISAGREE

INDECISIVE 
DO YOU KNOW OR HAVE YOU SEEN THIS PERSON IN THE PHOTOGRAPH BEFORE?

$\square \quad$ YES $\square$ NO

M-1. THIS PERSON'S PHYSICAL APPEARANCE IS:

VERY PHYSICALLY

ATTRACT IVE

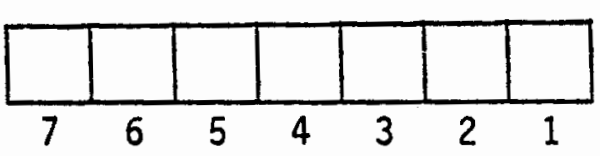

VERY PHYSICALLY

UNATTRACTIVE

M-2. THIS PERSON'S HAIR IS:

VERY PHYSICALLY

ATTRACTIVE

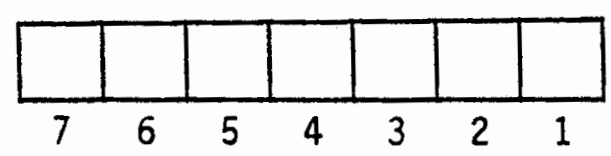

VERY PHYSICALLY

UNATTRACTIVE

M-3. THIS PERSON'S FACIAL EXPRESSION IS:

VERY PHYSICALLY

ATTRACTIVE

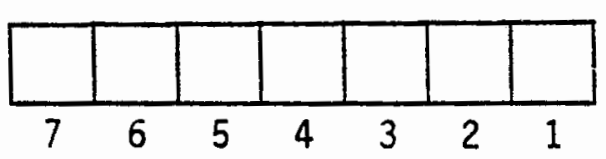

VERY PHYSICALLY

UNATTRACTIVE

M-4. THIS PERSON'S HEALTH IS:

VERY PHYSICALLY

ATTRACTIVE

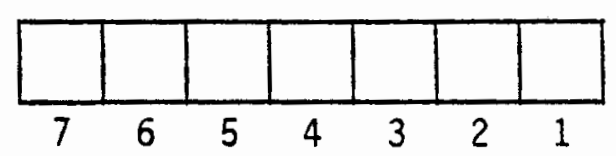

VERY PHYSICALLY UNATTRACTIVE

M-5. THIS PERSON'S EYES ARE:

VERY PHYSICALLY ATTRACTIVE

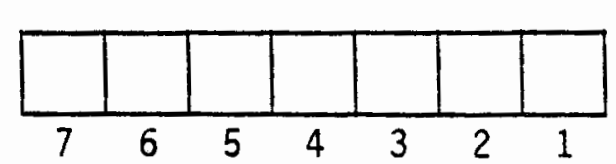

VERY PHYSICALLY UNATTRACTIVE 
M-6. THIS PERSON'S IS WELL GROOMED:

VERY PHYSICALLY ATTRACTIVE

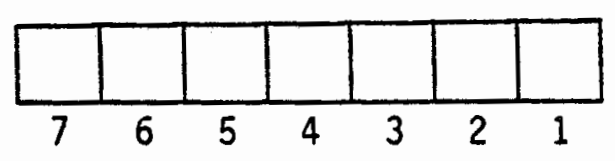

VERY PHYSICALLY UNATTRACTIVE

$M-7$.

THIS PERSON'S SKIN IS:

VERY PHYSICALLY ATTRACTIVE

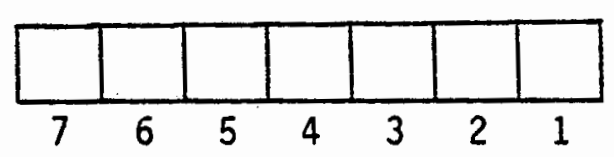

VERY PHYSICALLY UNATTRACTIVE

M-8.

THIS PERSON'S SMILE IS:

VERY PHYSICALLY ATTRACTIVE

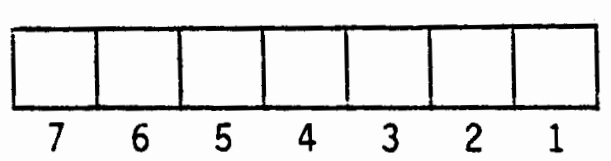

VERY PHYSICALLY UNATTRACTIVE

M-9. THIS PERSON'S FACIAL GROOMING IS:

VERY PHYSICALLY ATTRACTIVE

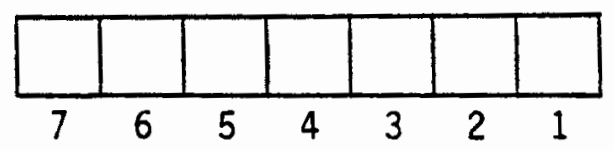

M-10. THIS PERSON'S IS:

VERY PHYSICALLY ATTRACTIVE

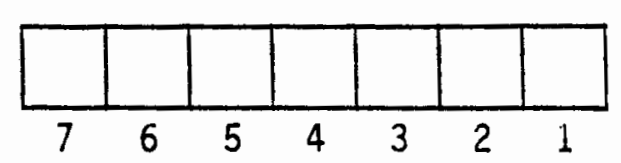

VERY PHYSICALLY UNATTRACTIVE 
SECTION II

I would like you to answer how you feel on the following 10 items consisting of descriptive traits (ranging from opposite to opposite) about the person in the photograph.

M-11. THIS PERSON APPEARS:

ACTIVE

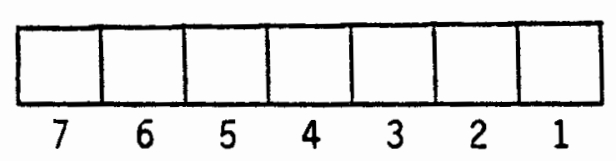

PASSIVE

M-12. THIS PERSON APPEARS:

CONSIDERATE

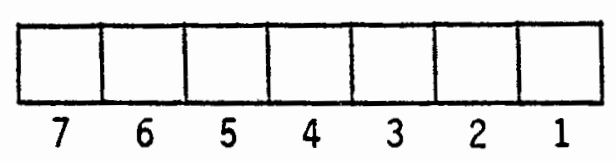

INCONS IDERATE

M-13. THIS PERSON APPEARS:

ENTHUSIASTIC

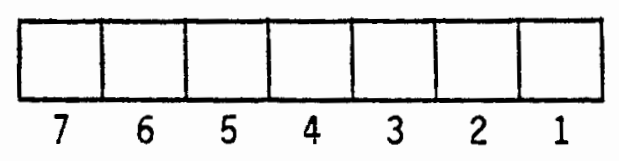

UNENTHUSIASTIC

M-14. THIS PERSON APPEARS:

PLEASANT

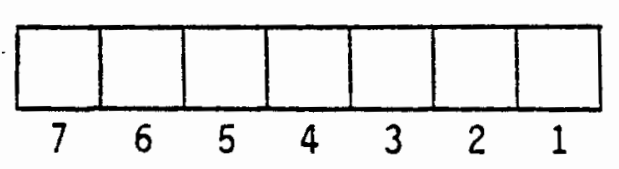

UNPLEASANT

M-15. THIS PERSON APPEARS:

STRONG

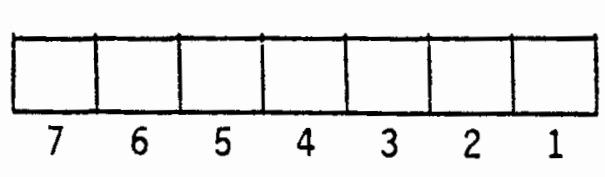

WEAK 
M-16. THIS PERSON APPEARS:

FLEXIBLE

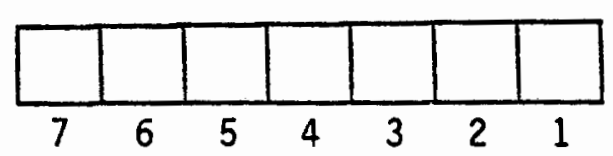

INFLEXIBLE

M-17. THIS PERSON APPEARS:

AMBITIOUS,

HAROW ORKING

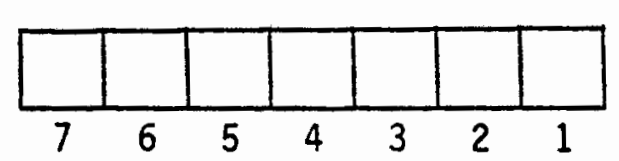

UNAMBITIOUS,

LAZY

M-18. THIS PERSON APPEARS:

UNDERSTANDING

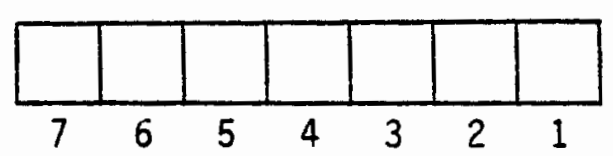

NOT UNDERSTANDING

M-19. THIS PERSON APPEARS:

ALERT

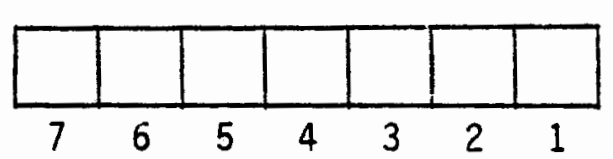

NONALERT

M-20. THIS PERSON APPEARS:

COOPERATIVE

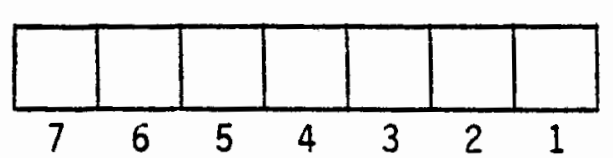

UNCOOPERATIVE 
SECTION III.

Another important part of this study is to learn more about interpersonal behavior. I would like you to indicate your degree of agreement with each of the folloiwng statements by checking how you feel.

M-21. I FEEL I WOULD LIKE THIS PERSON.

STRONGLY AGREE

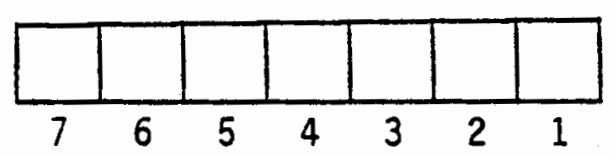

STRONGLY DISAGREE

M-22. I BELIEVE I WOULD ENJOY HAVING A FRIENDLY CHAT WITH THIS PERSON.

STRONGLY AGREE

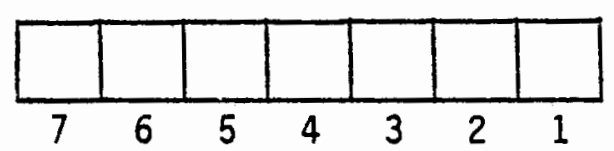

STRONGLY DISAGREE

M-23. THIS PERSON APPEALS TO ME.

STRONGLY AGREE

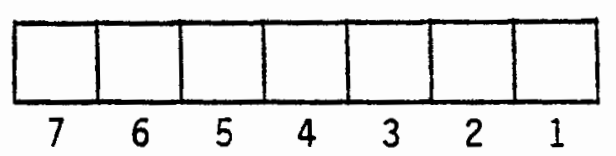

STRONGLY DISAGREE

M-24. IF I WERE WITH THIS PERSON, I WOULD PROBAELY INITIATE A CONVERSATION WITH HIM/HER.

STRONGLY AGREE

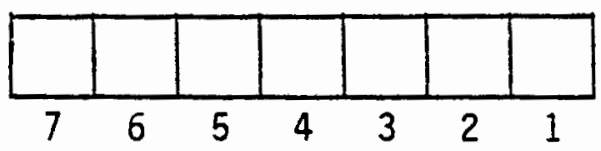

M-25 I THINK I WOULD ADMIRE THIS PERSON.

STRONGLY AGREE

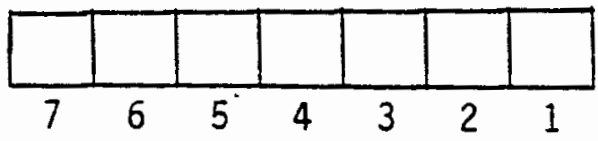

STRONGLY DISAGREE

STRONGLY DISAGREE 
M-26. I WOULD CONSIDER HAVING THIS PERSON AS A CLOSE, INTIMATE FRIEND.

STRONGLY AGREE

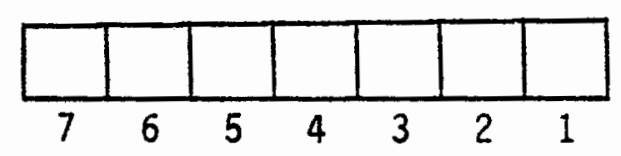

STRONGLY DISAGREE

M-27. I AM INTERESTED IN KNONING THIS PERSON.

STRONGLY AGREE

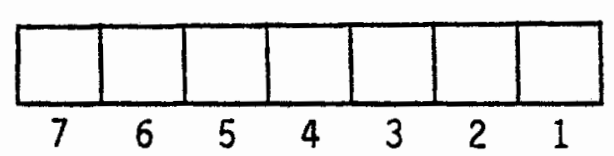

STRONGLY DISAGREE

M-28. I WOULD GLADLY RESPOND TO THIS PERSON IF HE/SHE INITIATED A CONVERSATION.

STRONGLY AGREE

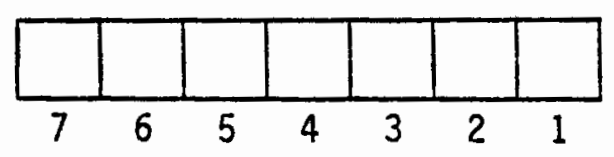

STRONGLY DISAGREE

M-29. I AM ATTRACTED TO THIS PERSON.

STRONGLY AGREE

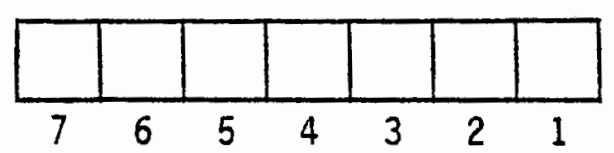

STRONGLY DISAGREE

M-30. I THINK I WOULD ENJOY SPENDING AN AFTERNOON WITH THIS PERSON.

STRONGLY AGREE

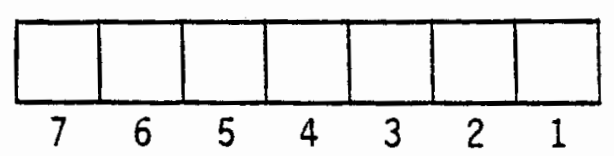

STRONGLY DISAGREE 


\section{APPENDIX E}

QUESIONNAIRE DEVELOPMENT 
1. General Statistics

Total

Test 1

Post test

2. Frequency Run

SEXSS by Q7 to Q111

AGESS by $Q 7$ to Q111

3. Reliabilities: 1) Correlation Matrix; 2) Alpha \#

PHYS (Physical) Q1 to Q22

Retest

Total

INSTRU (Instrumental Qualities) $023,25,27, .29,31,33,35,96$, 98,102

Test

Retest

Total

INTER (Interpersonal Qualities) Q24, 26, 28, 30, 32, 34, 36, 95, $97,99,100,104,105$

Test

Retest

Total

AFFECT Q39, 42, 45, 48, 51, 54, 57, 60,63, 66, 69 72, 75, 78, 81, Test $84,87,90,101,103$

Retest

Total

BEH PRE (Behavior Predisposition) Q 37, 38, 40, 41, 43, 44, 46, $47,49,50,52,53,55,56,58,59,61,62,64,65,67,68$, $70,71,73,74,76,77,79,80,82,83,85,86,88,89,91$,

Test Retest $92,93,94$

Total

4.

FACTOR ANALYSIS: 1) Correlation Matraix; 2) Factor loading

PHYSICAL - TOTAL, TEST, RETEST

INSTRUMENTAL QUALITIES - TOTAL, TEST, RETEST

INTERPERSONAL QUALITIES - TOTAL, TEST, RETEST

AFFECT - TOTAL, TEST, RETREST

BEHAVIOR PREDISPOSITION - TOTAL, TEST, RETEST

5. PEARSON $r$ CORRELATION BETWEEN ITEMS ON TEST VS. RETEST

EX: Q 7 VS R7

PHYSICAL (TOTAL)

INSTRUMENTAL QUALITIES (TOTAL)

INTERPERSONAL QUALITIES (TOTAL)

AFFECT (TOTAL)

BEHAVIOR PREDISPOSITION (TOTAL)

Figure 11. Statistical analysis used on the Honeywell 66/40 computer to validate the original OFIQ AND MFIQ items. 
APPENDIX $F$

CODESHEETS 
SOCIAL ECONOMIC STATUS (SES) QUESTIONNAIRE CODING SHEET

\begin{tabular}{|c|c|c|c|}
\hline $\begin{array}{l}\text { Column } \\
\text { No. }\end{array}$ & $\begin{array}{l}\text { Phase } \\
\text { IV }\end{array}$ & $\begin{array}{l}\text { Question No. } \\
\text { Coded AS On Question. As }\end{array}$ & Question \\
\hline $1-3$ & $\mathrm{~F}-1$ & $Q-1$ & Card No. 1 \\
\hline $4-5$ & & & Blank \\
\hline $6-9$ & $F-2$ & $Q-2$ & Subjects ID No. \\
\hline 10 & $F-3$ & $Q-3$ & $\begin{array}{l}\text { Type of Participant } \\
1 \text { SS (Subject 1) } \\
2 \text { Panel of Judges } \\
\text { (Subject 2) } \\
3 \text { Pre-test of } \\
\text { Instrument (Sub- } \\
\text { ject } 3 \text { ) } \\
4 \text { Subject } 4 \\
0 \text { MI/NA (Missing } \\
\text { Information/ } \\
\text { Not Applicable) }\end{array}$ \\
\hline
\end{tabular}

\begin{tabular}{|c|c|c|c|c|}
\hline 11 & $F-4$ & $Q-4$ & & $\begin{array}{l}\text { Age Group That } \\
\text { Respondent Has } \\
\text { Evaluated: } \\
1 \text { Young } \\
2 \text { Middle Group } \\
3 \text { old } \\
4 \text { No one } \\
5 \text { Young/Middle/01d } \\
0 \mathrm{MI} / \mathrm{NA}\end{array}$ \\
\hline 12 & $F-5$ & $Q-5$ & Q-1 & $\begin{array}{ll}\text { Your Sex: } & 1 \text { Male } \\
& 2 \text { Female }\end{array}$ \\
\hline 13 & $F-6$ & $Q-6$ & & $\begin{array}{l}\text { Age Group of } \\
\text { Participant: } \\
1 \text { Young } \\
2 \text { Middle Group } \\
3 \text { old }\end{array}$ \\
\hline $14-15$ & $F-7$ & Q-7 & $Q-2$ & $\begin{array}{l}\text { Your Present Age: } \\
\text { No. of Chrono- } \\
\text { logical Years. }\end{array}$ \\
\hline 16 & $F-8$ & Q-8 & $Q-3$ & $\begin{array}{l}\text { Are you Presently: } \\
1 \text { Employed full time } \\
2 \text { Employed part time } \\
3 \text { Unemployed } \\
4 \text { Retired } \\
0 \text { MI/NA }\end{array}$ \\
\hline
\end{tabular}




\begin{tabular}{|c|c|c|c|c|}
\hline $\begin{array}{l}\text { Col umn } \\
\text { No. }\end{array}$ & $\begin{array}{l}\text { Phase } \\
\text { IV }\end{array}$ & $\begin{array}{r}Q \\
\text { Coded AS }\end{array}$ & $\begin{array}{l}\text { stion No. } \\
\text { On Question. As }\end{array}$ & Question \\
\hline 17 & $F-9$ & Q-9 & $Q-4$ & $\begin{array}{l}\text { Please Describe your } \\
\text { Occupation: (See } \\
\text { Occupational Classi- } \\
\text { fication 1-8) } \\
0 \mathrm{MI} / \mathrm{NA}\end{array}$ \\
\hline 18 & $F-10$ & $Q-9 A$ & $Q-4 A$ & $\begin{array}{l}\text { Please Describe your } \\
\text { Spouse' Occupation: } \\
\text { (See Occupational } \\
\text { Classification 1-8) } \\
0 \mathrm{MI} / \mathrm{NA}\end{array}$ \\
\hline $19-20$ & $F-11$ & $Q-10$ & Q5 & $\begin{array}{l}\text { What was your approx. } \\
\text { Gross Family Income } \\
\text { From all resources, } \\
\text { before taxes in } 1980 \text { ? } \\
1 \text { Less than } \$ 4,999 \\
25,000 \text { to } 8,999 \\
39,000 \text { to } 12,999 \\
413,000 \text { to } 16,999 \\
517,000 \text { to } 20,999 \\
621,000 \text { to } 24,999 \\
725,000 \text { to } 28,999 \\
8 \text { over } \$ 29,000 \\
0 \mathrm{Mi} / \mathrm{NA}\end{array}$ \\
\hline 21 & $F-12$ & Q-11 & $Q-6$ & $\begin{array}{l}\text { Which is the highest } \\
\text { level of education } \\
\text { you have completed? } \\
1 \text { No formal education } \\
2 \text { Some grade school } \\
3 \text { Completed grade } \\
\text { school } \\
4 \text { Some high school } \\
5 \text { Completed high } \\
\text { school } \\
6 \text { Some college } \\
7 \text { Completed college } \\
8 \text { Some graduate work } \\
0 \text { MI/NA }\end{array}$ \\
\hline 22 & $F-13$ & $0-12$ & Q-7 & $\begin{array}{l}\text { Are you presently? } \\
1 \text { Not a college/ } \\
\text { university student } \\
2 \text { Enrolled part time } \\
\text { as a college/ } \\
\text { university } \\
\text { student }\end{array}$ \\
\hline
\end{tabular}




\begin{tabular}{|c|c|c|c|}
\hline $\begin{array}{l}\text { Col umn } \\
\text { No. }\end{array}$ & $\begin{array}{l}\text { Phase } \\
\text { IV }\end{array}$ & $\begin{array}{l}\text { Question No. } \\
\text { Coded As On Question. As }\end{array}$ & Question \\
\hline & & & $\begin{array}{l}3 \text { Enrolled full time } \\
\text { as a college/ } \\
\text { university student } \\
4 \text { Not enrolled as a } \\
\text { college/univ. } \\
\text { student, but work- } \\
\text { ing on thesis/ } \\
\text { dissertation } \\
\text { MI/NA }\end{array}$ \\
\hline 23 & $F-14$ & $Q-13$ & $\begin{array}{l}\text { Sexuality: } \\
\text { Homosexual } 1-7 \\
\text { Heterosexual } \\
0 \mathrm{MI} / \mathrm{NA}\end{array}$ \\
\hline
\end{tabular}

Dillman, Don A. (1978). Mail and telephone surveys: The

total design method, New York: John Wiley \& Sons, Inc., p. 135. 
ORIGINAL FIRST IMPRESSION QUESTIONNAIRE (OFIQ)

\begin{tabular}{lll}
$\begin{array}{l}\text { Column } \\
\text { No. }\end{array}$ & $\begin{array}{c}\text { Question No. } \\
\text { Coded As On Q As }\end{array}$ & Question \\
\hline $1-3$ & $Q-1$ & Card No. 1 \\
\hline 4 & $0-2$ & Test No.: $\begin{array}{l}1 \text { Test } \\
2 \text { Retest } \\
0 \mathrm{MI} / \mathrm{NA}\end{array}$ \\
\end{tabular}

\begin{tabular}{|c|c|c|c|}
\hline 5 & & Bl ank & \\
\hline $6-9$ & Q-3 & Identification No. & \\
\hline $10-13$ & Q-4 & Photo Identification & No. (Photo) \\
\hline 14 & Q-5 & Age Group of Photo: & $\begin{array}{ll}1 & \text { Young } \\
2 & \text { Middle } \\
3 & \text { old } \\
0 & \mathrm{MI} / \mathrm{NA}\end{array}$ \\
\hline
\end{tabular}

\begin{tabular}{lll}
\hline $15 \quad Q-6 \quad$ Sex of Photo; & 1 male \\
& 2 female \\
& $0 \mathrm{MI} / \mathrm{NA}$ \\
& \\
\end{tabular}

\begin{tabular}{llll}
\hline 16 & $0-7$ & I-1 & $\begin{array}{l}\text { Physical Appearance: } \\
\text { Attractive 7--1 Unattractive } \\
0 \mathrm{MI} / \mathrm{NA}\end{array}$ \\
\hline 17 & $0-8$ & $1-2$ & $\begin{array}{l}\text { Nose: } \\
\text { Attractive 7--1 Unattractive } \\
0 \mathrm{MI} / \mathrm{NA}\end{array}$ \\
\hline
\end{tabular}

\begin{tabular}{llll}
\hline 18 & $0-9$ & I-3 & $\begin{array}{l}\text { Face Shape: } \\
\text { Attractive 7--1 Unattractive } \\
\text { O MI/NA }\end{array}$ \\
\hline 19 & $0-10$ & I-4 & $\begin{array}{l}\text { Eyebrows: } \\
\text { Attractive 7--1 Unattractive } \\
\text { O MI/NA }\end{array}$ \\
\hline 20 & $0-11$ & I-5 & $\begin{array}{l}\text { Facial grooming: } \\
\text { Attractive 7--1 Unattractive } \\
\text { O MI/NA }\end{array}$ \\
\hline 21 & $0-12$ & I-6 & $\begin{array}{l}\text { Smile: } \\
\text { Attractive 7--1 Unattractive } \\
\text { 0 MI/NA }\end{array}$ \\
\hline
\end{tabular}




\begin{tabular}{clll}
$\begin{array}{c}\text { Column } \\
\text { No. }\end{array}$ & \multicolumn{2}{c}{ Question No. } \\
Coded As & On $Q$ As & Question \\
\hline 22 & Q-13 & I-7 & $\begin{array}{l}\text { Weight } \\
\text { Attractive } \\
0 \text { MI/NA }\end{array}$
\end{tabular}

\begin{tabular}{lll}
\hline 23 & Q-14 I-8 & Teeth: \\
Attractive 7--1 Unattractive \\
$0 \mathrm{MI} / \mathrm{NA}$
\end{tabular}

\begin{tabular}{lll}
\hline $24-15$ & I-9 & $\begin{array}{l}\text { Hairstyle: } \\
\text { Attractive 7--1 Unattractive } \\
0 \mathrm{MI} / \mathrm{NA}\end{array}$
\end{tabular}

\begin{tabular}{|c|c|c|c|}
\hline 25 & $Q-16$ & $\mathrm{I}-10$ & $\begin{array}{l}\text { Personal Hygiene: } \\
\text { Attractive } 7--1 \text { Unattractive } \\
0 \mathrm{MI} / \mathrm{NA}\end{array}$ \\
\hline
\end{tabular}

\begin{tabular}{lll}
\hline $26-17$ & I-11 & Chin: \\
Attractive 7--1 Unattractive & OMI/NA
\end{tabular}

\begin{tabular}{lll}
\hline $27-18$ & $\mathrm{I}-12$ & $\begin{array}{l}\text { Eyes: } \\
\text { Attractive } \\
0 \mathrm{MI} / \mathrm{NA}\end{array}$ \\
& & \\
&
\end{tabular}

\begin{tabular}{lll}
\hline 28 & $0-19 \quad$ I-13 & $\begin{array}{l}\text { Posture: } \\
\text { Attractive } \\
\\
0 \mathrm{MI} / \mathrm{NA}\end{array}$ \\
&
\end{tabular}

\begin{tabular}{|c|c|c|c|}
\hline 29 & $Q-20$ & I-14 & $\begin{array}{l}\text { Mouth: } \\
\text { Attractive 7--1 Unattractive } \\
0 \mathrm{MI} / \mathrm{NA}\end{array}$ \\
\hline 30 & Q-21 & I-15 & $\begin{array}{l}\text { Facial Expression: } \\
\text { Attractive } 7--1 \text { Unattractive } \\
0 \mathrm{MI} / \mathrm{NA}\end{array}$ \\
\hline 31 & Q-22 & I-16 & $\begin{array}{l}\text { Skin: } \\
\text { Attractive 7--1 Unattractive } \\
0 \mathrm{MI} / \mathrm{NA}\end{array}$ \\
\hline 32 & $Q-23$ & I-17 & $\begin{array}{l}\text { Ears: } \\
\text { Attractive 7--1 Unattractive } \\
0 \mathrm{MI} / \mathrm{NA}\end{array}$ \\
\hline 33 & $Q-24$ & I-18 & $\begin{array}{l}\text { Health: } \\
\text { Attractive 7--1 Unattractive } \\
0 \mathrm{MI} / \mathrm{NA}\end{array}$ \\
\hline
\end{tabular}


Column Question No.

No. Coded As on Q As Question

\begin{tabular}{|c|c|c|c|}
\hline 34 & Q-25 & I -19 & $\begin{array}{l}\text { Neck: } \\
\text { Attractive 7--1 Unattractive } \\
0 \mathrm{MI} / \mathrm{NA}\end{array}$ \\
\hline 35 & $Q-26$ & $I-20$ & $\begin{array}{l}\text { Hair: } \\
\text { Attractive 7--1 Unattractive } \\
0 \mathrm{MI} / \mathrm{NA}\end{array}$ \\
\hline 36 & Q-27 & I -21 & $\begin{array}{l}\text { Well Groomed: } \\
\text { Strongly Agree 7--1 Strongly } \\
0 \mathrm{MI} / \mathrm{NA}\end{array}$ \\
\hline
\end{tabular}

\begin{tabular}{|c|c|c|c|}
\hline 37 & $Q-28$ & $I-22$ & $\begin{array}{l}\text { Physical Appaerance: } \\
\text { Attractive } 7--1 \text { Unattractive } \\
0 \mathrm{MI} / \mathrm{NA}\end{array}$ \\
\hline
\end{tabular}

$0 \mathrm{MI} / \mathrm{NA}$

\begin{tabular}{lll}
\hline $38-29$ & I-23 & $\begin{array}{l}\text { Independence: } \\
\text { Independent } 7--1 \text { Dependent } \\
\text { O MI/NA }\end{array}$
\end{tabular}

\begin{tabular}{lll}
\hline $39 \quad$ Q-30 & I-24 & Attitude: \\
& Pleasant & 7--1 Unpleasant \\
& 0 MI/NA
\end{tabular}

\begin{tabular}{llll}
\hline 40 & Q-31 & I-25 & $\begin{array}{l}\text { Active: } \\
\text { Active 7--1 Passive } \\
\text { 0 MI/NA }\end{array}$ \\
\hline 41 & Q-32 & I-26 & $\begin{array}{l}\text { Open Mindedness: } \\
\text { Open Minded 7--1 Closed Minded } \\
\text { 0 MI/NA }\end{array}$ \\
\hline 42 & Q-33 & I-27 & $\begin{array}{l}\text { Enthusiasm: } \\
\text { Enthusiastic 7--1 Unenthusiastic } \\
\text { 0 MI/NA }\end{array}$ \\
\hline 43 & Q-34 & I-28 & $\begin{array}{l}\text { Consideration: } \\
\text { Considerate 7--1 Inconsiderate } \\
\text { 0 MI/NA }\end{array}$ \\
\hline 44 & Q-35 & I-29 & $\begin{array}{l}\text { Speed: } \\
\text { Fast 7--1 Slow } \\
\text { 0 MI/NA }\end{array}$ \\
\hline 45 & Q-36 & I-30 & $\begin{array}{l}\text { Loving (Caring): } \\
\text { Loving 7--1 Unioving } \\
\text { 0 MI/NA }\end{array}$ \\
\hline
\end{tabular}




\begin{tabular}{|c|c|c|c|}
\hline $\begin{array}{l}\text { Column } \\
\text { No. }\end{array}$ & $\begin{array}{r}\text { Que } \\
\text { Coded }\end{array}$ & $\begin{array}{l}\text { No. } \\
\text { On Q As }\end{array}$ & Question \\
\hline 46 & $Q-37$ & $I-31$ & $\begin{array}{l}\text { Strength: } \\
\text { Strong } 7--1 \text { Weak } \\
0 \mathrm{MI} / \mathrm{NA}^{2}\end{array}$ \\
\hline
\end{tabular}

\begin{tabular}{llll}
\hline 47 & $0-38$ & I-32 & $\begin{array}{l}\text { Cooperation: } \\
\text { Cooperative 7--1 Uncooperati } \\
0 \text { MI/NA }\end{array}$ \\
\hline 48 & $0-39$ & I-33 & $\begin{array}{l}\text { Ambition: } \\
\text { Ambitious, Hardworking 7--1 } \\
\text { Unambitious, Lazy } \\
\text { O MI/NA }\end{array}$ \\
& &
\end{tabular}

\begin{tabular}{lll}
\hline $49-40$ & $1-34$ & $\begin{array}{l}\text { Understanding: } \\
\text { Understanding } \\
0 \text { MI/NA }\end{array}$ \\
& &
\end{tabular}

\begin{tabular}{lll}
\hline $50-41$ & I-35 & $\begin{array}{l}\text { Alertness: } \\
\text { Alert } 7--1 \text { Non Alert } \\
0 \text { MI/NA }\end{array}$ \\
& \\
\hline
\end{tabular}

\begin{tabular}{|c|c|c|c|}
\hline 51 & Q-42 & $I-36$ & $\begin{array}{l}\text { Flexibility: } \\
\text { Flexible } 7--1 \text { Inflexible } \\
0 \mathrm{MI} / \mathrm{NA}\end{array}$ \\
\hline
\end{tabular}

\begin{tabular}{lll}
\hline 52 & Q-43 I-37 & $\begin{array}{l}\text { Enjoy a friendly chat with this } \\
\text { person. } \\
\text { Strongly Agree } 7--1 \text { Strongly Di sagree } \\
\end{array}$ \\
& 0 MI/NA
\end{tabular}

\begin{tabular}{|c|c|c|c|}
\hline 53 & Q-44 & $I-38$ & $\begin{array}{l}\text { Would talk more frequently then usual } \\
\text { SS. } \\
\text { Strongly Agree } 7--1 \text { Strongly Disagree } \\
0 \mathrm{MI} / \mathrm{NA}\end{array}$ \\
\hline
\end{tabular}

\begin{tabular}{|c|c|c|c|}
\hline 54 & $Q-45$ & I-39 & $\begin{array}{l}\text { Would enjoy knowing this person. } \\
\text { Strongly Agree } 7--1 \text { Strongly Disagree } \\
0 \mathrm{MI} / \mathrm{NA}\end{array}$ \\
\hline
\end{tabular}

\begin{tabular}{|c|c|c|c|}
\hline 55 & $Q-46$ & $I-40$ & $\begin{array}{l}\text { If in an informal conversation with } \\
\text { SS. I would be inclined to dominate } \\
\text { it. } \\
\text { Strongly Agree } 7--1 \text { Strongly Disagree } \\
0 \mathrm{MI} / \mathrm{NA}\end{array}$ \\
\hline 56 & $Q-47$ & $I-41$ & $\begin{array}{l}\text { If I spoke to this person, I probably } \\
\text { pause quite often in the conversation } \\
\text { Strongly Agree } 7--1 \text { Strongly Disagree } \\
0 \mathrm{MI} / \mathrm{NA}\end{array}$ \\
\hline
\end{tabular}




\begin{tabular}{|c|c|c|c|}
\hline $\begin{array}{l}\text { Column } \\
\text { No. }\end{array}$ & $\begin{array}{r}\text { Que } \\
\text { Coded }\end{array}$ & $\begin{array}{l}\text { No. } \\
\text { On Q As }\end{array}$ & Question \\
\hline 57 & $Q-48$ & $I-42$ & $\begin{array}{l}\text { I am attracted to this person. } \\
\text { Strongly Agree } 7--1 \text { Strongly Di sagree } \\
0 \mathrm{MI} / \mathrm{NA}\end{array}$ \\
\hline 58 & $Q-49$ & $I-43$ & $\begin{array}{l}\text { If I were having a small dinner party, } \\
\text { I would be pleased to invite SS. } \\
\text { Strongly Agree } 7--1 \text { Strongly Disagree } \\
0 \mathrm{MI} / \mathrm{NA}\end{array}$ \\
\hline
\end{tabular}

\begin{tabular}{|c|c|c|c|}
\hline 59 & $Q-50$ & I-44 & $\begin{array}{l}\text { I would gladly respond to SS if he/she } \\
\text { initiated a conversation. } \\
\text { Strongly Agree } 7--1 \text { Strongly Disagree } \\
0 \mathrm{MI} / \mathrm{NA}\end{array}$ \\
\hline
\end{tabular}

\begin{tabular}{|c|c|c|c|}
\hline 60 & $Q-51$ & $I-45$ & $\begin{array}{l}\text { I think I would admire this Ss. } \\
\text { Strongly Agree } 7--1 \text { Strongly Di sagree } \\
0 \mathrm{MI} / \mathrm{NA}\end{array}$ \\
\hline
\end{tabular}

\begin{tabular}{|c|c|c|c|}
\hline 61 & $Q-52$ & $I-46$ & $\begin{array}{l}\text { If I saw SS, I would probably } \\
\text { acknowl edge him/her when passing. } \\
\text { Strongly Agree } 7--1 \text { Strongly Di sagree } \\
0 \mathrm{MI} / \mathrm{NA}\end{array}$ \\
\hline
\end{tabular}

\begin{tabular}{|c|c|c|c|}
\hline 62 & $Q-53$ & I-47 & $\begin{array}{l}\text { I think I would enjoy spending a } \\
\text { week's vacation with SS. } \\
\text { Strongly Agree } 7--1 \text { Strongly Di sagree } \\
0 \mathrm{MI} / \mathrm{NA}\end{array}$ \\
\hline
\end{tabular}

\begin{tabular}{|c|c|c|c|}
\hline 63 & $Q-54$ & I -48 & $\begin{array}{l}\text { I think I could feel close to SS. } \\
\text { Strongly Agree } 7--1 \text { Strongly Di sagree } \\
0 \mathrm{MI} / \mathrm{NA}\end{array}$ \\
\hline
\end{tabular}

\begin{tabular}{|c|c|c|c|}
\hline 64 & Q-55 & $I-49$ & $\begin{array}{l}\text { If in a social conversation with SS, } \\
\text { would not be inclined to let SS get } \\
\text { in last word. } \\
\text { Strongly Agree } 7--1 \text { Strongly Disagree } \\
0 \mathrm{MI} / \mathrm{NA}\end{array}$ \\
\hline
\end{tabular}

\begin{tabular}{|c|c|c|c|}
\hline 65 & Q-56 & $I-50$ & $\begin{array}{l}\text { I would call SS by his/her first name. } \\
\text { Strongly Agree } 7--1 \text { Strongly Di sagree } \\
0 \mathrm{MI} / \mathrm{NA}\end{array}$ \\
\hline
\end{tabular}

\begin{tabular}{|c|c|c|c|}
\hline 66 & $Q-57$ & $I-51$ & $\begin{array}{l}\text { I feel favorable towards knowing SS. } \\
\text { Strongly Agree } 7--1 \text { Strongly Di sagree } \\
0 \mathrm{MI} / \mathrm{NA}\end{array}$ \\
\hline
\end{tabular}




\begin{tabular}{|c|c|c|c|}
\hline $\begin{array}{l}\text { Column } \\
\text { No. }\end{array}$ & $\begin{array}{r}\text { Que } \\
\text { Codec }\end{array}$ & $\begin{array}{l}\text { No. } \\
\text { On Q As }\end{array}$ & Question \\
\hline 67 & $Q-58$ & $I-52$ & $\begin{array}{l}\text { If I were having a 1-to-1 conversation } \\
\text { with SS, I would tend to let him/her } \\
\text { talk more than half the time. } \\
\text { Strongly Agree } 7--1 \text { Strongly Disagree } \\
0 \mathrm{MI} / \mathrm{NA}\end{array}$ \\
\hline
\end{tabular}

\begin{tabular}{|c|c|c|c|}
\hline 68 & Q-59 & $I-53$ & $\begin{array}{l}\text { I would tend to hesitate before } \\
\text { speaking to SS. } \\
\text { Strongly Agree } 7--1 \text { Strongly Disagree } \\
0 \mathrm{MI} / \mathrm{NA}\end{array}$ \\
\hline
\end{tabular}

\begin{tabular}{lll}
\hline $69-60$ & I-54 & SS appeals to me \\
Strongly Agree $7--1$ Strongly Disagree \\
$0 \mathrm{MI} / \mathrm{NA}$
\end{tabular}

\begin{tabular}{llll}
\hline 70 & $0-61$ & I-55 & $\begin{array}{l}\text { I think I would enjoy inviting SS to } \\
\text { my home for dinner. } \\
\text { Strongly Agree 7--1 Strongly Disagree } \\
\text { 0 MI/NA }\end{array}$ \\
\hline 71 & Q-62 & I-56 & $\begin{array}{l}\text { In speaking to SS, I would tend to } \\
\text { talk for long periods of time. } \\
\text { Strongly Agree 7--1 Strongly Disagree } \\
\text { OMI/NA }\end{array}$
\end{tabular}

\begin{tabular}{|c|c|c|c|}
\hline 72 & $Q-63$ & $I-57$ & $\begin{array}{l}\text { I think I would feel amiable towards } \\
\text { SS. } \\
\text { Strongly Agree } 7--1 \text { Strongly Disagree } \\
0 \mathrm{MI} / \mathrm{NA}\end{array}$ \\
\hline
\end{tabular}

\begin{tabular}{lll}
\hline 73 & Q-64 I-58 & If I were to speak with SS, I would be \\
inclined to let him/her start the \\
conversation. \\
Strongly Agree $7--1$ Strongly Disagree \\
OMI/NA
\end{tabular}

\begin{tabular}{|c|c|c|c|}
\hline 74 & $Q-65$ & $I-59$ & $\begin{array}{l}\text { I think I would enjoy spending an } \\
\text { afternoon with SS. } \\
\text { Strongly Agree } 7--1 \text { Strongly Disagree } \\
0 \mathrm{MI} / \mathrm{NA}\end{array}$ \\
\hline
\end{tabular}

\begin{tabular}{|c|c|c|c|}
\hline 75 & Q-66 & $I-60$ & $\begin{array}{l}\text { I am interested in knowing SS. } \\
\text { Strongly Agree } 7--1 \text { Strongly Disagree } \\
0 \mathrm{MI} / \mathrm{NA}\end{array}$ \\
\hline 76 & Q-67 & $I-61$ & $\begin{array}{l}\text { If I were with SS, I would rely on } \\
\text { him/her to keep the conversation } \\
\text { going. } \\
\text { Strongly Agree } 7--1 \text { Strongly Disagree } \\
0 \mathrm{MI} / \mathrm{NA}\end{array}$ \\
\hline
\end{tabular}




\begin{tabular}{lll}
$\begin{array}{c}\text { Column } \\
\text { No. }\end{array}$ & \multicolumn{2}{c}{ Question No. } \\
\hline 77 & Qoded As & \multicolumn{1}{c}{ Qn Q As }
\end{tabular}

\begin{tabular}{|c|c|c|c|}
\hline 78 & Q-69 & I -63 & $\begin{array}{l}\text { I feel I can identify with SS. } \\
\text { Strongly Agree } 7--1 \text { Strongly Disagree } \\
0 \mathrm{MI} / \mathrm{NA}\end{array}$ \\
\hline
\end{tabular}

\begin{tabular}{|c|c|c|c|}
\hline 79 & $Q-70$ & I-64 & $\begin{array}{l}\text { Most people would react favorably to } \\
\text { SS. } \\
\text { Strongly Agree } 7--1 \text { Strongly Disagree } \\
0 \mathrm{MI} / \mathrm{NA}\end{array}$ \\
\hline
\end{tabular}

\begin{tabular}{|c|c|c|c|}
\hline 80 & Q-71 & I-65 & $\begin{array}{l}\text { I would prefer to speak to SS rather } \\
\text { than to listen to him/her. } \\
\text { Strongly Agree } 7--1 \text { Strongly Di sagree } \\
0 \mathrm{MI} / \mathrm{NA}\end{array}$ \\
\hline
\end{tabular}

\begin{tabular}{|c|c|c|}
\hline $1-3$ & $Q-1$ & Card No. 2 \\
\hline 4 & $Q-2$ & $\begin{array}{ll}\text { Test No.: } & 1 \text { Test } \\
& 2 \text { Retest } \\
& 0 \text { MI/NA }\end{array}$ \\
\hline
\end{tabular}

\begin{tabular}{|c|c|c|c|}
\hline 5 & & & Blank \\
\hline $6-9$ & $Q-3$ & & Identification No. \\
\hline $10-13$ & $Q-4$ & & Photo Identification No. \\
\hline 14 & Q-72 & $I-66$ & $\begin{array}{l}\text { I would feel warmly towards SS. } \\
\text { Strongly Agree } 7--1 \text { Strongly Di sagree } \\
0 \mathrm{MI} / \mathrm{NA}\end{array}$ \\
\hline 15 & Q-73 & $I-67$ & $\begin{array}{l}\text { I would consider having SS as a close, } \\
\text { intimate, friend. } \\
\text { Strongly Agree } 7 \text {--1 Strongly Disagree } \\
0 \mathrm{MI} / \mathrm{NA}\end{array}$ \\
\hline 16 & $Q-74$ & $I-68$ & $\begin{array}{l}\text { If I were with SS in a social conver- } \\
\text { sation, I would direct the course } \\
\text { of it. } \\
\text { Strongly Agree } 7--1 \text { Strongly Disagree } \\
0 \text { MI/NA }\end{array}$ \\
\hline
\end{tabular}




\begin{tabular}{|c|c|c|c|}
\hline $\begin{array}{l}\text { Col umn } \\
\text { No. }\end{array}$ & $\begin{array}{l}\text { Que } \\
\text { Codec }\end{array}$ & $\begin{array}{l}\text { No. } \\
\text { On Q As }\end{array}$ & Question \\
\hline 17 & $Q-75$ & $I-69$ & $\begin{array}{l}\text { I wish I could meet SS. } \\
\text { Strongly Agree } 7--1 \text { Strongly Di sagree } \\
0 \mathrm{MI} / \mathrm{NA}\end{array}$ \\
\hline
\end{tabular}

\begin{tabular}{|c|c|c|c|}
\hline 18 & $Q-76$ & $\mathrm{I}-70$ & $\begin{array}{l}\text { On a job, I believe I would enjoy } \\
\text { having SS as a co-worker. } \\
\text { Strongly Agree } 7--1 \text { Strongly Disagree } \\
0 \mathrm{MI} / \mathrm{NA}\end{array}$ \\
\hline
\end{tabular}

\begin{tabular}{|c|c|c|c|}
\hline 19 & Q-77 & $\mathrm{I}-71$ & $\begin{array}{l}\text { If I wre having a conversation with } \\
\text { SS, I would probably speak shorter } \\
\text { periods of time than usual. } \\
\text { Strongly Agree } 7--1 \text { Strongly Disagree } \\
0 \text { MI/NA }\end{array}$ \\
\hline 20 & $Q-78$ & I-72 & $\begin{array}{l}\text { I think this SS subject is similar to } \\
\text { me. } \\
\text { Strongly Agree } 7--1 \text { Strongly Disagree } \\
0 \mathrm{MI} / \mathrm{NA}\end{array}$ \\
\hline
\end{tabular}

\begin{tabular}{|c|c|c|c|}
\hline 21 & Q-79 & $\mathrm{I}-73$ & $\begin{array}{l}\text { In speaking with SS, I feel I could } \\
\text { express myself quite freely. } \\
\text { Strongly Agree } 7--1 \text { Strongly Disagree } \\
0 \mathrm{MI} / \mathrm{NA}\end{array}$ \\
\hline 22 & Q-80 & I -74 & $\begin{array}{l}\text { If speaking to SS, I would probably } \\
\text { let him/her talk for long periods } \\
\text { of time. } \\
\text { Strongly Agree } 7--1 \text { Strongly Disagree } \\
0 \mathrm{MI} / \mathrm{NA}\end{array}$ \\
\hline
\end{tabular}

\begin{tabular}{lll}
\hline $23-81$ & I-75 & $\begin{array}{l}\text { This person is repul sive to me. } \\
\text { Strongly. Agree } 7--1 \text { Strongly Disagree } \\
0 \mathrm{MI} / \mathrm{NA}\end{array}$
\end{tabular}

\begin{tabular}{|c|c|c|c|}
\hline 24 & $Q-82$ & I-76 & $\begin{array}{l}\text { In conversing with SS, I would have } \\
\text { the tendency to let him/her determine } \\
\text { the course of the conversation. } \\
\text { Strongly Agree } 7--1 \text { Strongly Disagree } \\
0 \text { MI/NA }\end{array}$ \\
\hline
\end{tabular}

\begin{tabular}{|c|c|c|c|}
\hline 25 & $Q-83$ & $\mathrm{I}-77$ & $\begin{array}{l}\text { If I were with SS, I would probably } \\
\text { initiate a conversation with him/her. } \\
\text { Strongly Agree } 7--1 \text { Strongly Disagree } \\
0 \mathrm{MI} / \mathrm{NA}\end{array}$ \\
\hline 26 & Q-84 & I -78 & $\begin{array}{l}\text { I feel I could become friends with SS. } \\
\text { Strongly Agree } 7--1 \text { Strongly Disagree } \\
0 \mathrm{MI} / \mathrm{NA}\end{array}$ \\
\hline
\end{tabular}


Column Question No.

No. Coded As On Q As Question

\begin{tabular}{|c|c|c|c|}
\hline 27 & Q-85 & $I-79$ & $\begin{array}{l}\text { If I were in a social conversation } \\
\text { with SS, I would probably come on } \\
\text { strong. } \\
\text { Strongly Agree } 7--1 \text { Strongly Disagree } \\
0 \mathrm{MI} / \mathrm{NA}\end{array}$ \\
\hline
\end{tabular}

\begin{tabular}{|c|c|c|c|}
\hline 28 & $Q-86$ & $I-80$ & $\begin{array}{l}\text { If I were on a bus, I would probably } \\
\text { sit next to SS. } \\
\text { Strongly Agree } 7--1 \text { Strongly Disagree } \\
0 \mathrm{MI} / \mathrm{NA}\end{array}$ \\
\hline
\end{tabular}

\begin{tabular}{|c|c|c|c|}
\hline 29 & Q-87 & I -81 & $\begin{array}{l}\text { I would feel fond of SS. } \\
\text { Strongly Agree } 7--1 \text { Strongly Disagree } \\
0 \mathrm{MI} / \mathrm{NA}\end{array}$ \\
\hline 30 & Q-88 & I -82 & $\begin{array}{l}\text { If I were in a social conversation } \\
\text { with SS, I would speak quite } \\
\text { frequently. } \\
\text { Strongly Agree } 7--1 \text { Strongly Disagree } \\
0 \mathrm{MI} / \mathrm{NA}\end{array}$ \\
\hline
\end{tabular}

\begin{tabular}{lll}
\hline 31 & Q-89 I-83 & If I were speaking to SS, I would try \\
& to take charge of things in our \\
& conversation. \\
& Strongly Agree $7--1$ Strongly Disagree \\
& $0 \mathrm{MI} / \mathrm{NA}$
\end{tabular}

\begin{tabular}{llll}
\hline 32 & Q-90 & I-84 & $\begin{array}{l}\text { I would love to meet SS. } \\
\text { Strongly Agree 7--1 Strongly Disagree } \\
\text { O MI/NA }\end{array}$ \\
\hline 33 & Q-91 & I-85 & $\begin{array}{l}\text { If I were having trouble in my family, } \\
\text { I would confide in SS. } \\
\text { Strongly Agree 7--1 Strongly Disagree } \\
\text { 0 MI/NA }\end{array}$
\end{tabular}

\begin{tabular}{|c|c|c|c|}
\hline 34 & Q-92 & $\mathrm{I}-86$ & $\begin{array}{l}\text { If I wre having a conversation with } \\
\text { SS, I would probably be inclined to } \\
\text { talk more forceful than usual. } \\
\text { Strongly Agree } 7--1 \text { Strongly Disagree } \\
0 \mathrm{MI} / \mathrm{NA}\end{array}$ \\
\hline 35 & Q-93 & I-87 & $\begin{array}{l}\text { I feel I would dislike knowing SS. } \\
\text { Strongly Agree } 7--1 \text { Strongly Di sagree } \\
0 \mathrm{MI} / \mathrm{NA}\end{array}$ \\
\hline
\end{tabular}




\begin{tabular}{|c|c|c|c|c|}
\hline $\begin{array}{l}\text { Column } \\
\text { No. }\end{array}$ & $\begin{array}{l}\text { Questio } \\
\text { Coded As }\end{array}$ & $\begin{array}{l}\text { No. } \\
\text { On Q As }\end{array}$ & & Question \\
\hline 36 & Q-94 & $I-88$ & $\begin{array}{l}\text { If I were } \\
\text { with SS, } \\
\text { to let } h \\
\text { Strongly } \\
0 \mathrm{MI} / \mathrm{NA}\end{array}$ & $\begin{array}{l}\text { in a social conversation } \\
\text { I would probably be inclined } \\
\text { im/her get in the last word. } \\
\text { Agree } 7--1 \text { Strongly Disagree }\end{array}$ \\
\hline
\end{tabular}

\begin{tabular}{|c|c|c|c|}
\hline 37 & Q-95 & I-89 & $\begin{array}{l}\text { In speaking to SS, I would probably } \\
\text { feel inhibited. } \\
\text { Strongly Agree } 7--1 \text { Strongly Disagree } \\
0 \mathrm{MI} / \mathrm{NA}\end{array}$ \\
\hline
\end{tabular}

\begin{tabular}{lll}
\hline $38 \quad 0-96$ & I-90 & I feel I would like SS. \\
& Strongly Agree 7--1 Strongly Disagree \\
& $0 \mathrm{MI} / \mathrm{NA}$
\end{tabular}

\begin{tabular}{|c|c|c|c|}
\hline 39 & Q-97 & $I-91$ & $\begin{array}{l}\text { If I spoke to SS, I would prefer to } \\
\text { keep my comments brief. } \\
\text { Strongly Agree } 7--1 \text { Strongly Disagree } \\
0 \mathrm{MI} / \mathrm{NA}\end{array}$ \\
\hline
\end{tabular}

\begin{tabular}{|c|c|c|c|}
\hline 40 & Q-98 & I -92 & $\begin{array}{l}\text { I would be inclined to jump into } \\
\text { informal conversations with SS. } \\
\text { Strongly Agree } 7--1 \text { Strongly Disagree } \\
0 \mathrm{MI} / \mathrm{NA}\end{array}$ \\
\hline
\end{tabular}

\begin{tabular}{|c|c|c|c|}
\hline 41 & $Q-99$ & I-93 & $\begin{array}{l}\text { In speaking with SS, I would feel I } \\
\text { could not express my views. } \\
\text { Strongly Agree } 7--1 \text { Strongly Disagree } \\
0 \mathrm{MI} / \mathrm{NA}\end{array}$ \\
\hline 42 & $Q-100$ & I -94 & $\begin{array}{l}\text { If I wre in a social conversaton with } \\
\text { SS, I would probably dominate it. } \\
\text { Strongly Agree } 7--1 \text { Strongly Disagree } \\
0 \mathrm{MI} / \mathrm{NA}\end{array}$ \\
\hline
\end{tabular}

\begin{tabular}{llll}
\hline 43 & Q-101 & I-95 & $\begin{array}{l}\text { Kind 7--1 Cruel } \\
0 \text { MI/NA }\end{array}$ \\
\hline 44 & Q-102 & I-96 & $\begin{array}{l}\text { Intelligent 7--1 Unintelligent } \\
0 \text { MI/NA }\end{array}$ \\
\hline 45 & Q-103 & I-97 & $\begin{array}{l}\text { Modest 7--1 Immodest } \\
0 \text { MI/NA }\end{array}$ \\
\hline 46 & Q-104 & I-98 & $\begin{array}{l}+ \text { Self Concept 7--1 - Self Concept } \\
0 \text { MI/NA }\end{array}$ \\
\hline
\end{tabular}


Column Question No.

No. Coded As on Q As Question

47 Q-105 I-99 Sensitive 7--1 Insensitive

$0 \mathrm{MI} / \mathrm{NA}$

\begin{tabular}{llll}
\hline 48 & Q-106 & I-100 & $\begin{array}{l}\text { Sincere } \\
0 \mathrm{MI} / \mathrm{NA}\end{array}$
\end{tabular}

\begin{tabular}{llll}
\hline $49-107$ & I-101 & $\begin{array}{l}\text { Friendly 7--1 Unfriendly } \\
0 \mathrm{MI} / \mathrm{NA}\end{array}$
\end{tabular}

$50 \quad$ Q-108 I-103 Likeable 7--1 Unlikeable

$0 \mathrm{MI} / \mathrm{NA}$

\begin{tabular}{llll}
\hline 51 & Q-109 I-104 & $\begin{array}{l}\text { Sophisticated 7--1 Unsophisticated } \\
\text { OMI/NA }\end{array}$
\end{tabular}

\begin{tabular}{|c|c|c|c|}
\hline 52 & $Q-110$ & $I-105$ & $\begin{array}{l}\text { Warm } 7--1 \text { Cold } \\
0 \mathrm{MI} / \mathrm{NA}\end{array}$ \\
\hline
\end{tabular}

\begin{tabular}{llll}
\hline 53 & Q-111 & I-102 & Tidy 7--1 Untidy \\
& 0 MI/NA
\end{tabular}

Note: The five components defining Physical Attractiveness operational definitions were the following:

Physical Attributes -- external body attributes of a person.

Instrumental Attributes -- concerned with the non-social or internal aspects of personal ability; that is projected qualities such as kindness and warmth.

Interpersonal Qualities -- concerned with the social aspect of personal ability, that is the ability to get along with others.

Affect Qualities -- absolute liking.

Behavior Predisposition (BP) -- intent of the behavior. 
MODIFIED FIRST IMPRESSION QUESTIONNAIRE (MFIQ)

\begin{tabular}{|c|c|c|c|c|}
\hline $\begin{array}{l}\text { Column } \\
\text { No. }\end{array}$ & $\begin{array}{l}\text { Phase } \\
\text { Coded As }\end{array}$ & $\begin{array}{l}\text { IV } \\
\text { On Q As }\end{array}$ & $\begin{array}{l}\text { Phase III } \\
\text { Coded As On } Q \text { As }\end{array}$ & Question \\
\hline $1-3$ & $F-1$ & & $Q-1$ & Card No. 1 \\
\hline 4 & $F-2$ & $Q-2$ & & $\begin{array}{cl}\text { Test No. } & 1 \text { Test } \\
& 2 \text { Retest } \\
& 3 \text { Main Study } \\
& 0 \mathrm{MI} / \mathrm{NA}\end{array}$ \\
\hline
\end{tabular}

\begin{tabular}{|c|c|c|c|}
\hline 5 & & & $\begin{array}{l}\text { Photo Order: } 1,2,3, \\
4,5,6, \\
0 \mathrm{MI} / \mathrm{NA}\end{array}$ \\
\hline $6-9$ & $F-3$ & Q-3 & ID No. (Subjects) \\
\hline $10-13$ & $F-4$ & $Q-4$ & Photo ID No. \\
\hline 14 & $F-5$ & $Q-5$ & $\begin{array}{l}\text { Age Group of Photo: } \\
1 \text { Young } \\
2 \text { Middle } \\
3 \text { old } \\
0 \mathrm{Mi} / \mathrm{NA}\end{array}$ \\
\hline
\end{tabular}

\begin{tabular}{|c|c|c|c|c|c|c|}
\hline 15 & $F-6$ & Q-6 & & & $\begin{array}{l}\text { Sex of } \\
\text { Photo: }\end{array}$ & $\begin{array}{l}1 \text { Male } \\
2 \text { Female }\end{array}$ \\
\hline 16 & $F-7$ & $M-1$ & Q-7 & $I-1$ & $\begin{array}{r}\text { Physical } \\
\text { Attract } \\
\text { Unattr } \\
0 \mathrm{MI} / \mathrm{NA}\end{array}$ & $\begin{array}{l}\text { earance: } \\
7-1 \\
\text { ve }\end{array}$ \\
\hline
\end{tabular}

\begin{tabular}{|c|c|c|c|c|c|}
\hline 17 & $F-8$ & $M-2$ & $Q-26$ & I-20 & $\begin{array}{l}\text { Hair: } \\
\text { Attractive } 7-1 \\
\text { Unattractive } \\
0 \mathrm{MI} / \mathrm{NA}\end{array}$ \\
\hline
\end{tabular}

\begin{tabular}{|c|c|c|c|c|c|}
\hline 18 & $F-9$ & $M-3$ & $Q-21$ & $I-15$ & $\begin{array}{l}\text { Facial Expression: } \\
\text { Attractive } 7-1 \\
\text { Unattractive } \\
0 \mathrm{MI} / \mathrm{NA}\end{array}$ \\
\hline
\end{tabular}

\begin{tabular}{lllll}
\hline 19 & F-10 & M-4 & Q-24 & I-18 $\begin{array}{c}\text { Health: } \\
\text { Attractive 7-1 } \\
\text { Unattractive } \\
\text { O MI/NA }\end{array}$ \\
\hline 20 & F-11 & M-5 & Q-18 & I-12 $\begin{array}{l}\text { Eyes: } \\
\text { Attractive 7-1 } \\
\text { Unattractive } \\
\text { MI/NA }\end{array}$ \\
\hline
\end{tabular}


Column Phase IV Phase III

No. Coded As On Q As Coded As on Q As Question

\begin{tabular}{|c|c|c|c|c|c|}
\hline 21 & $F-12$ & $M-6$ & $Q-27$ & I-21 & $\begin{array}{l}\text { Wel1 Groomed: } \\
\text { Attractive } 7--1 \\
\text { Unattractive } \\
\text { O MI/NA }\end{array}$ \\
\hline
\end{tabular}

\begin{tabular}{|c|c|c|c|c|c|}
\hline 22 & $F-13$ & $M-7$ & Q-22 & I-16 & $\begin{array}{l}\text { Skin: } \\
\text { Attractive } 7--1 \\
\text { Unattractive } \\
0 \mathrm{MI} / \mathrm{NA}\end{array}$ \\
\hline
\end{tabular}

\begin{tabular}{|c|c|c|c|c|c|}
\hline 23 & $F-14$ & $M-8$ & $0-12$ & $I-6$ & $\begin{array}{l}\text { Smile: } \\
\text { Attractive 7--1 } \\
\text { Unattractive } \\
0 \mathrm{MI} / \mathrm{NA}\end{array}$ \\
\hline 24 & $F-15$ & $M-9$ & Q-11 & $I-5$ & $\begin{array}{l}\text { Facial Grooming: } \\
\text { Attractive } 7--1 \\
\text { Unattractive } \\
0 \mathrm{MI} / \mathrm{NA}\end{array}$ \\
\hline 25 & $F-16$ & $M-10$ & $Q-28$ & $I-22$ & $\begin{array}{l}\text { This person is: } \\
\text { Attractive } 7--1 \\
\text { Unattractive } \\
0 \mathrm{MI} / \mathrm{NA}\end{array}$ \\
\hline 26 & $F-17$ & $M-11$ & $Q-31$ & $I-25$ & $\begin{array}{l}\text { Active } \\
\text { Active } 7--1 \text { Pas } \\
0 \mathrm{MI} / \mathrm{NA}\end{array}$ \\
\hline
\end{tabular}

\begin{tabular}{|c|c|c|c|c|c|}
\hline 27 & $F-18$ & $M-12$ & Q-34 & I -24 & $\begin{array}{l}\text { Consideration } \\
\text { Considerate } 7-1 \\
\text { Inconsiderate } \\
0 \mathrm{MI} / \mathrm{NA}\end{array}$ \\
\hline 28 & $F-19$ & $M-13$ & $Q-33$ & $I-27$ & $\begin{array}{l}\text { Enthusiasm } \\
\text { Enthusiastic } 7--1 \\
\text { Unenthusiastic } \\
0 \mathrm{MI} / \mathrm{NA}\end{array}$ \\
\hline 29 & $F-29$ & $M-14$ & $Q-30$ & $\mathrm{I}-24$ & $\begin{array}{l}\text { Attitude } \\
\text { Pleasant } 7--1 \\
\text { Unpleasant } \\
0 \mathrm{MI} / \mathrm{NA}\end{array}$ \\
\hline
\end{tabular}

\begin{tabular}{|c|c|c|c|c|c|}
\hline 30 & $F-21$ & M-15 & Q-37 & I-3I & $\begin{array}{l}\text { Strength } \\
\text { Strong } 7--1 \text { Weak } \\
0 \text { MI/NA }\end{array}$ \\
\hline
\end{tabular}


Column Phase IV Phase III

No. Coded As on Q As Coded As on Q As Question

\begin{tabular}{ccccc}
\hline 31 & F-22 & M-16 & Q-42 & $\begin{array}{l}\text { I-36 } \\
\text { Flexibility } \\
\text { Flexible 7--1 } \\
\text { Unflexible } \\
\text { O MI/NA }\end{array}$ \\
\hline 32 & F-23 & M-17 & Q-39 & I-33 $\begin{array}{l}\text { Ambition } \\
\text { Hardworking 7--1 } \\
\text { Lazy } \\
0 \text { MI/NA }\end{array}$ \\
\hline 33 & F-24 & M-18 & Q-40 & I-34 $\begin{array}{l}\text { Understanding } \\
\text { Understanding 7--1 } \\
\text { Not Understanding } \\
\text { OMI/NA }\end{array}$ \\
\hline
\end{tabular}

\begin{tabular}{|c|c|c|c|c|c|}
\hline 34 & $F-25$ & $M-19$ & Q-41 & $I-35$ & $\begin{array}{l}\text { Alertness } \\
\text { Alert } 7--1 \text { Nonalert } \\
0 \mathrm{MI} / \mathrm{NA}\end{array}$ \\
\hline 35 & $F-26$ & $M-20$ & Q-38 & $I-32$ & $\begin{array}{l}\text { Cooperation } \\
\text { Cooperative } 7--1 \\
\text { Uncooperative } \\
0 \mathrm{MI} / \mathrm{NA}\end{array}$ \\
\hline 36 & $F-27$ & $M-21$ & Q-96 & I -90 & $\begin{array}{l}\text { I feel I would like } \\
\text { SS. } \\
\text { Strongly agree } 7--1 \\
\text { Strongly disagree } \\
0 \mathrm{MI} / \mathrm{NA}\end{array}$ \\
\hline 37 & $F-28$ & $M-22$ & Q-43 & I-37 & $\begin{array}{l}\text { I believe I would } \\
\text { enjoy having a } \\
\text { friendly chat with } \\
\text { SS. } \\
\text { Strongly agree } 7--1 \\
\text { Strongly disagree } \\
0 \mathrm{MI} / \mathrm{NA}\end{array}$ \\
\hline 38 & $F-29$ & $M-23$ & $Q-60$ & $I-54$ & $\begin{array}{l}\text { SS appeals to me. } \\
\text { Strongly agree } 7-- \\
\text { Strongly di sagree } \\
0 \mathrm{MI} / \mathrm{NA}\end{array}$ \\
\hline
\end{tabular}




\begin{tabular}{|c|c|c|c|c|c|}
\hline $\begin{array}{l}\text { Col umn } \\
\text { No. }\end{array}$ & $\begin{array}{l}\text { Phase } \\
\text { Coded As }\end{array}$ & $\begin{array}{l}\text { IV } \\
\text { On Q As }\end{array}$ & $\begin{array}{l}\text { Phase } \\
\text { Coded As }\end{array}$ & $\begin{array}{l}\text { II I } \\
\text { On Q As }\end{array}$ & Question \\
\hline 39 & $F-30$ & $M-24$ & Q-83 & $1-77$ & $\begin{array}{l}\text { If I were with SS-I } \\
\text { would probably } \\
\text { initiate a conver- } \\
\text { sation with SS. } \\
\text { Strongly agree 7--1 } \\
\text { Strongly disagree } \\
\text { OMI/NA }\end{array}$ \\
\hline
\end{tabular}

\begin{tabular}{|c|c|c|c|c|c|}
\hline ECT 40 & $F-31$ & $M-25$ & $Q-51$ & $I-45$ & $\begin{array}{l}\text { I think I would } \\
\text { admire SS. } \\
\text { Strongly agree } 7--1 \\
\text { Strongly disagree } \\
\text { OMI/NA }\end{array}$ \\
\hline
\end{tabular}

\begin{tabular}{|c|c|c|c|c|c|}
\hline 41 & $F-32$ & $M-26$ & Q-73 & $I-67$ & $\begin{array}{l}\text { I would consider } \\
\text { having SS a close } \\
\text { intimate friend. } \\
\text { Strongly agree 7-- } \\
\text { Strongly disagree } \\
0 \mathrm{MI} / \mathrm{NA}\end{array}$ \\
\hline ECT 42 & $F-33$ & $M-27$ & Q-66 & $I-60$ & $\begin{array}{l}\text { I am interested in } \\
\text { knowing SS. } \\
\text { Strongly agree } 7- \\
\text { Strongly disagree } \\
0 \mathrm{MI} / \mathrm{NA}\end{array}$ \\
\hline
\end{tabular}

\begin{tabular}{|c|c|c|c|c|c|}
\hline 43 & $F-34$ & $M-28$ & Q-50 & I -44 & $\begin{array}{l}\text { I would gladly } \\
\text { respond to SS if he/ } \\
\text { she initiated a } \\
\text { conversation. } \\
\text { Strongly agree } 7 \text { - } \\
\text { Strongly disagree } \\
\text { OMI/NA }\end{array}$ \\
\hline
\end{tabular}

\begin{tabular}{|c|c|c|c|c|}
\hline ECT 44 & $F-35$ & $M-29$ & $Q-48$ & $\begin{array}{c}\text { I-42 I am attracted to SS. } \\
\text { Strongly agree } 7 \text { - } \\
\text { Strongly disagree } \\
0 \mathrm{MI} / \mathrm{NA}\end{array}$ \\
\hline
\end{tabular}

\begin{tabular}{|c|c|c|c|c|c|}
\hline 45 & $F-36$ & $M-30$ & Q-65 & I -59 & $\begin{array}{l}\text { I think I would enjoy } \\
\text { spending an after- } \\
\text { noon with SS. } \\
\text { Strongly agree } 7- \\
\text { Strongly disagree } \\
0 \mathrm{MI} / \mathrm{NA}\end{array}$ \\
\hline
\end{tabular}


APPENDIX G

SELECTION JUDGES 
Transcription of Selection Judges Tape Recorded Responses

Young Male Judges Tape-recorded Responses Evaluating Male Stimulus Photographs Physical Attractiveness.

Young Stimulus Photographs:

Most Physically Attractive

LETTER T: Large piercing eyes, the way he holds his head inconjunction with his smile, it's a good photograph of the guy, he has high cheek bones, the shape of his face is kind of square with high cheek bones, he doesn't have a real large nose, mustache looks nice on him, he has a nice smile, dark eyes with dark hair, inconsideration together looks nice. you can tell he is a poor college student.... he's got straight teeth and a smile--he should be selling Marlboro cigarettes.

Least Physically Attractive

LETTER B: Oh God! His beard, hair, the look on his face, there is no smile in relationship to his hair the beard is kinky too... his hair is kinky that really makes his face look elongated. He looks just like an old friend of mine... He does. He look straggly.. he looks like he hasn't combed his hair for months...he hasn't trimmed his beard in six months. He's just let it grow and he doesn't look well kept--like this (sort of girl...she doesn't look like she takes real good care of herself. I'm kind of interested why he doesn't smile.. maybe because he does not brush his teeth. He doesn't have any smile whatsoever. His hair because it is kinky..it makes his forehead look twice as long as it normally would then if it came down and the beard accents the high forehead.. Poor guy!

Middle-Aged Stimulus Photographs:

Most Physically Attractive

LETTER FF: I like the combination of gray with blue, light blue, his suit and his shirt and the tie makes a good combination of color. About him, his face is not real strange... he's smiling a little bit..his hair is well combed.

Least Physically Attractive Male:

LETTER P: His face looks like he is afraid of something, his hair is not well combed. His eyeglasses are not straight, like his eyebrow is higher on one side then the other..his smile is really weird.. I like the color combination of red and blue-- that he is wearing. 
0ld Stimulus Photographs:

Most Physically Attractive Male

LETTER HH: It is probably the way he looks, it looks like he carries himself well. He has definite features... a mustache that stands out.. He is serious looking..that's about it.

Least Physically Attractive Male

LETTER 00: His whole expression.. looks like he is kind of lost. His hair is kind of thrown back. He doesn't look very old but he looks older than he probably is. Has big ears. It looks like he has not slept in a couple of weeks. 
Young Male Selection Judges Tape-recorded Responses Evaluating Female Stimulus Photographs Physical Attractiveness.

Young Stimulus Photographs:

Most Physically Attractive

LETTER M: Her eyes, her really big eyes--they are pretty, her hair...I really like long hair, fairly nice smile...that's it. She's got a nice oval face... it doesn't look like she is overweight.

Least Physically Attractive

LETTER S: Her hair is greasy, she has squint little eyes and real large eyebrows and she is definitely overweight. Her hair is not right, even though it is greasy and it is not attractive... it kind of hangs there and starts to look cluttered ...vomit all I can say.... Letter $M$ has long hair..just as Letter $S$ does but it is shaped around her face and it does$n ' t$ look quite as greasy and it looks like she takes care of herself better. It looks like she cares more about her personal appearance than the other person (Letter $S$ ). Letter $M$ doesn't have hair behind her ears and it looks ugly on this other girl (Letter $S$ ). Letter $S$ face is kind of fat and long and that's all I can say about that poor girl.

Middle-Aged Stimulus Photographs:

Most Physically Attractive

She's smiling, that attracts me...her eyes are really nice ...her hair is combed and well styled, I think. Maybe the red color of her dress and the color of her lips and her teeth--they are straight and her lips are red.... you know the lips.... the chin and the nose.

Least Physically Attractive

LETTER DD: First of all, her hair is uncombed and not well set. She wears eyeglasses, her mouth is kind of unstraight, then the photograph is pretty dark and her shirt is not.... well put it's like open in the middle.

01d Stimulus Photographs:

Most Physically Attractive

LETTER MM: Probably her narrow definition of her face...probably the richness she portrays that makes her look like she is kind of glamorous. 
Least Physically Attractive

LETTER EE: No definite features...everything is just kind of rounded. Her hair is a mess... That's about it. 
Middle-Aged Male Selection Judges Tape Recorded Responses Evaluating Female Stimulus Photographs Physícal Attractiveness.

Young Stimulus Photographs:

Most Physically Attractive

LETTER M: It may have to do with the facial expression, the kind of look of openness and the way the eyes are looking. Al so, the features are basically regular which I suppose is one characteristic and I like the way her hair looks and the general appearance of openness.

Least Physically Attractive

LETTER S: This is partly, I think an artifact of the photograph but the skin color is not particularly good and her face looks a bit heavy, her eyes are looking off instead of looking directly at you and maybe I'm not sure I'm responding to the general shape of her head or the combination of that and the hair style. I don't find any of these people particularly attractive.

Middle-Aged Stimulus Photographs:

Most Physically Attractive

LETTER BB: Generally, the shape of the face, the hair, the eyes and the kind of look on her face is pleasant.

Least Physically Attractive

LETTER I: The look on her face and to me the shape of her face. The hair color is alright looking. Again, I would not call her an unattractive person but of the ones I had to pick from, she is the one of the least physically attractive. The expression on her face.

0ld Stimul us Photographs:

Most Physically Attractive

LETTER AAA: He's clean cut.. None of them are particularly overweight. The eyes are looking at you. For one thing, he reminds me of my brother. There are two rules of people: what is in here and what is up here. Appearance is.....all of them look clean. Their all clean looking.

Least Physicall Attractive

LETTER 00: His appearance and he is not smiling, his eyes are...the rest of the photographs appear friendly. 
01d Male Selection Judges Tape-recorded Responses Evaluating Male Stimulus Photographs Physical Attractiveness.

Young Stimulus Photographs:

Most Physically Attractive

LETTER D: He looks like he would make a good football player. He looks like he might like to have a good time and be the life of the party. He looks like he might be interesting to know.

Least Physically Attractive

LETTER N: He has the super silliest look about him and that he might not be to nice to know. He looks like he has a weak personality and he doesn't have very strong features in his face.

Middle-Aged Stimulu Photographs:

Most Physically Attractive

LETTER W: The same thing holds true as Letter BB. That is an infectious picture whereas the other is a tantalizing picture and that is why the least physically attractive was not picked. Letter $W$ has an infectious grin and it looks like he is having some fun. Maybe, I would get along with him and maybe I wouldn't.

Least Physically Attractive

LETTER FF: He goes right along with the gal on the bottom, Letter I. He's not mean but he is very straight, he does not have a particular sense of humor as far as I can see.... I never saw an architect or a painter that looks like that. Maybe... he is a salesman... That's why.

01d Stimulus Photographs:

Most Physically Attractive

LETTER BB: Projection of a sense of warmth and a sense of humor.

Least Physically Attractive

LETTER UU: Fat--that's enough in the sense to find her unattractive. 
01d Male Selection Judges Tape-recorded Responses Evaluating Female Stimulus Photographs Physical Attractiveness.

Young Stimulus Photographs:

Most Physically Attractive

LETTER G: Very nice teeth, smile, intelligent look about her....eyes and her hair is attractive.

Least Physically Attractive

LETTER S: It does not look like a very tidy person with her unkept hair and because of her obesity..... that leads me to believe that she likes to eat alot. It might be unbecoming to her. It has a tendency to show up in her personality..... I believe...her looks. The type of person she is with her unkept hair. It looks like a mop and she looks like she likes junk food.

Middle-Aged Stimulus Photographs:

Most Physically Attractive

LETTER BB: Nothing physically, they are all very ordinary people. I... she has a good complexion, nice face, it's what is happening to that face that interests me and the same thing goes for the next one. You know what? What they are saying in their face that is intriguing. There is not a classic beauty in the lot.

Least Physically Attractive

LETTER I: She is sagging and I can't be bothered with people who are sagging. They tend to sag on me and I don't need that... She looks unhappy.. I don't need to borrow trouble. I have enough of my own.

0ld Stimulus Photographs:

Most Physically Attractive

LETTER BB: Her neatness....maybe it is someone that reminds me of.....in my past. Physical features, appearance not overweight I suppose are the most obvious. That's about it. She doesn't look like my wife either.

Least Physically Attractive

LETTER TT: The evidence of overweight, that's one thing that bothers me the most. I wouldn't say the hair or the facial

expression....no just the weight. 
01d Female Selection Judges Tape-recorded Responses Evaluating Male Stimulus Photographs Physical Attractiveness.

Young Stimulus Photographs:

Most Physically Attractive

LETTER N: Nothing in particular. I couldn't find in all the men, one that I really liked. He has a sparkle in his eyes. I am not satisfied with my first choice. He looks healthly.

Least Physically Attractive

LETTER D: I don't like the way he has his mouth open like that---it's kind of a crooked smile. Yet, I'm sure he's not the worst but he is the one I have in my hand.

Middle-Aged Stimulus Photographs:

Most Physically Attractive

LETTER W: He seems to have a nice smile and his eyes seem to smile as well. . It seems to be that he doesn't find it hard to do. I suppose he just has a pleasant..friendly look about him... that's what I assume to enjoy about all people I like best. He looks unpretentious looking, he doesn't have himself all gussied up. He is an ordinary man but a pleasant one.

Least Physically Attractive

LETTER FF: This man, he has a slightly foxy look. I think...he might be a little tricky but that could be perhaps..... I recall people that way that he reminds me of. Other than that I guess..neat, pleasant enough looking man...he's not unattractive but I i ike $W$ better.

01d Stimulus Photographs:

Most Physically Attractive

LETTER YY: He is neat, he doesn't look like an egotistical.....perhaps less egotistical than the majority of people... A good twinkle in his eyes that would indicate a good sense of humor. He doesn't have much hair but I'm sure he offsets it with his intelligence.

Least Physically Attractive

LETTER 00: He seems to be neat and all that...his appearance doesn't appeal to me. Well, maybe it's his seriousness or hair do--it's sort of the structure of his face and it doesn't look like he's got a good sense of humor. Also, his ears stick out. 
01d Female Selection Judges Tape-recorded Responses Evaluating Female stimulus Photographs Physical Attractiveness.

Young Stimulus Photographs:

Most Physically Attractive

LETTER K: She looks pleasant, healthy, very pretty, nice smile and I was just instantly attracted to her.

Least Physically Attractive

LETTER AA: She looks emaciated and I didn't like her grin--it's a little bit repulsive. She is probably an attractive girl to someone else. Also, she has that kind of shine on her face.... see...... I don't like that. Her hair is pushed up on one end and out of order.

Middle-Aged Stimulus Photographs:

Most Physically Attractive

LETTER EE: She has a calm, look, pleasant look, her mouth looks like she might smile quite....fairly often. She doesn't have a turn down look on her mouth. She's neat looking and all around pleasant. Someone you feel you could approach and get her response from her. She's not outstandingly beautiful. She is a neat, pleasant, friendly..looking woman.

Least Physically Attractive

LETTER DD: I think...perhaps the fact she is looking down a bit and her face is tilted down rather than looking up...possibly why. This is not the best pose of her this is the reason why the picture doesn't do her justice. She's not a homely person but of..... it could be that her hair do isn't quite becoming, pose, she is sort of grining, smiling a little sideways.. that might be a nervous habit but other than that she's not unpleasant looking. She doesnt' come on as positive in appearance as Letter EE does.

0ld Stimulus Photographs:

Most Physically Attractive

LETTER KK: She is very neat, pleasant smile, has a twinkle in her eyes, her hair is well groomed and I would evaluate her as having a good sense of humor, intelligent with a lust for life.

Least Physically Attractive

LETTER TT: Her obesity, she is neat as any obese person can be. She must have a good sense of humor---most heavy set people do...but I find mostly obese people unattractive. 
Young Female Selection Judges Tape-recorded Responses Evaluating Male Stimulus Photographs Physical Attractiveness.

Young Stimulus Photographs:

Most Physically Attractive

LETTER D: I like his clean cuttness and his whole outlook....just looks more cleancut..more than others, he just seems to be more down to earth and more natural.... the whole picture. I guess I can not think of one specific thing that I like about him, it is just his whole outlook.

Least Physically Attractive

LETTER L: His glasses, I don't think they are....look very attractive on him and the way he is bowing his head. He has a double chin.

Middle-Aged Stimulus Photographs:

Most Physically Attractive

LETTER $X$ : It's amazing that he has almost the same eyeglasses that my husband has .... the same color and style of hair, the same complexion but the only thing that is different is the mustache, essentially the same eye color. Besides from the fact he looks like my husband, he has a pleasant smile, the softness about his eyes, he doesn't have hard look about his eyes..he has a causal look..his hair is not combed and sprayed and dupped out of place or cuffs it to stick up. and..al though he is clean shaving, it looks as though there is some shadow in....generally a nice person you would like to be around.

Least Physically Attractive

LETTER Y: On God! This is something that should of stayed on the farm. He's grossly overweight, coming out of his shirt, a little smiling and it looks like it hurts to smile. He is losing his hair and that does not appeal to me. It looks like he needs a haircut even though he is losing most of it. The back is curling up and that is just tacky in my opinion. What can I say... I just find him offensive...just his appearance. Other than that, he is probably a very pleasant person. 
01d Stimulus Photographs:

Most Physically Attractive

LETTER BBB: Again, he has a friendly type of face.. of his oval face..his big nose, he's got a lot of hair and his brown eyes.

Least Physically Attractive

LETTER 00: I guess a combination of things.. which would be his receding hairline and his ears kind of stick out a little bit and I guess his slanted eyes too. 
Young Female Selection Judges Tape-recorded Responses Evaluating Female Stimulus Photographs Physical Attractiveness.

Young Stimulus Photographs:

Most Physically Attractive

LETTER G: I believe ..possibly.. the sparkle in her eyes and her curly hair and she seems to be or like herself and happy about herself so that's what I like about her. I think it is just the whole person that appeals to me and no specific thing.

Least Physically Attractive

LETTER S: Maybe the straightness in her hair and the more plumpness she seems to be. She just seems to be less appealing to me than the other person in picture G. I like her smile and I like her eyes that are open..... and facing me and maybe that's why I think she's unattractive.

Middle-Aged Stimulus Photographs:

Most Physically Attractive

LETTER C: The quality of the skin, it seems to have a nice luster to $i t$, the moist, well applied lipstick enhances the lips, the pleasant smile, there is a light in the eyes that come through, the hair is nicely done as to being cut and styled. Some jewelry on her ears and that adds to the pleasantness. Just a nice complexion and nice pleasant features not to obtusive or overhearing or underdone. The motherly type.

Least Physically Attractive

LETTER I: Oh my god! It looks like something left over from last Christmas. Her hair reminds me of someone who has a Mr. Potatoe head and taken a straight pin and stuck a piece of hair on it. It just is hard to imagine what really coming out of her skull. The neck, you can see the lines and the throat... the esophagus...her hair...skin is lined more than the other picture---she looks sunken in. And the glasses tend to hide any light that might be shining out of her eyes that is if she is alive. Looking at this picture, it can be undoubtful. This... if she were lying down could be the final picture before they put her under. I see no light in her eyes, there is no jewelry, she has no lip stick on--she looks gone but that color doesn't do anything for her. And the disshoveled hair and even the color of clothing ... she has on makes her look drab! Even the eyeglasses seem to enhance the hollowed out nature of her cheek bones.... the underneath portion keeps adding up to ugly, ugly, 
ugly.... The background behind her is darker than the background behind the prettier picture.

0ld Stimulus Photographs:

Most Physically Attractive

LETTER KK: She has a very pleasing face, a very warm face. The gray hair.. she portrays a very warm grandma like personality... I think.

Least Physically Attractive

LETTER TT: I guess, the obesity. I think that is most of it and the glasses. The hair looks nice but I think it is her obesity and her glasses. 
Middle-Aged Selection Male Judges Tape-recorded Responses Evaluating Male Stimulus Photographs Physical Attractiveness.

Young Stimulus Photographs:

Most Physically Attractive

LETTER T: I guess again, it has to do with the particular posture of the head and it may have to do with the regular features or the shape of the features. I guess I like the relationship of the amount of hair he has in relationship to the shape of his face, the eyes are good looking, it's kind of an alert look, that's what I would say.

Least Physically Attractive

LETTER R: I guess it has something to do with the relationship of the heaviness of his face--I think again the eyes are looking right at me which I like. I would not define this person on an absolute scale as being unattractive. I guess I am responding to the weight and the shape of the facial plain. In the photograph there is sort of a flatness--that I'm not sure would be the case with the person.

Middle-Aged Stimulus Photographs:

Most Physically Attractive

LETTER X: Well, it has to do with the shape of her face, the eyes, the hair and the shape of the hair. The guy looks nice with a mustache and that is what is attractive about him.

Least Physically Attractive

LETTER U: Again, it would be the expression on his face and the shape of his face and that sort of thing. As I have mentioned to you, you could of taken, the last five photos, $Z$ B Y P U and put them in any order and it would of been approximatly the same--it would of been for the same reason, I guess... Facial expression, the shape of his face, the eyes perhaps and not that any of them are unpleasant individuals---not that I would have difficulty dealing with anyone of them.

old Stimulus Photographs:

Most Physically Attractive

LETTER AAA: Projection of sense of humor--things of that nature. Least Physically Attractive

LETTER TT: Reflection of stereotypical person that you would find in mental illness or a detoxication center. 
Middle-Aged Female Selection Judges Tape-recorded Responses evaluating Female Stimulus Photographs Physical Attractiveness.

Young Stimulus Photographs:

Most Physically Attractive

LETTER M: Well, basically, I think she has a pretty smile--maybe the shape of her smile, I think her smile, other than her hair. She is not all made up but she looks like a clean cut young girl.

Least Physically Attractive

LETTER F: In the first place, her hair--I think... And really it is not.... She looks heavy but if something was done to her hair and to her eyes. It's her hair... it doesn't even look clean... really. That's what it reminds me of, somebody who doesn't care. She just needs a whole going over. I think if she.... she doesn't have a bad smile but that is all I can say for her. She doesn't look like a well groomed person.

Middle-Aged Stimulus Photographs:

Most Physically Attractive

LETTER H: There is some influence by the expression that she has an amicable expression on her face. From just bare appearance.... somebody who cares enough about her appearance to project an attractive image. There's definitely.... not stunningly.... but something about her that makes you think you would like her. She's not outstandingly beautiful.

Least Physically Attractive

LETTER C: It's really interesting, it's.... I don't find any of the females really unattractive. It's in fact....looking at her and the most physically attractive one...there isn't much difference. If you took off the glasses I'm sure there wouldn't be much difference, whatsoever. If you frizz her hair up....maybe it is that I don't find that style of hair attractive on the woman--as some people. It's not the glasses. Maybe an off-stear look, the coloring of her clothes --seems to go with her skin color. Maybe I have kind of a fundamental religious type look which I would tend to shy away with...but I don't find any of the woman unattractive. I find them all attractive in different ways. 
0ld Stimulus Photographs:

Most Physically Attractive

LETTER WW: She just has a sparkle about her, she looks bright eyed and bushy tailed.... She's got a nice smile... she looks perky and has kind eyes. She just looks like a nice lady. She was probably physically attractive when she was younger. That's about it.

Least Physically Attractive

LETTER TT: Mainly, because she is so much overweight which is an unattractive quality. That is about the main thing, her obesity...otherwise she looks like a bright lady, probably kind of jolly and alot of fun. It is hard to say, because people can be different from your impressions. 
Middle-Aged Female Selection Judges Tape-recorded Responses Evaluating Male Stimulus Photographs Physical Attractiveness.

Young Stimulus Photographs:

Most Physically Attractive

LETTER N: I think he looks like a nice young boy. I don't like mustaches but that is the only thing I don't like about him. He just looks like a nice young boy and I see alot of them. He just looks like a nice kid. He looks like a nice clean cut kid.

Least Physically Attractive

LETTER B: His beard, his mustache, his expression on his face...he might be a nice kid too but I just of... but maybe because he is not smiling--he is just unattractive..probably both the beard and hair. He probably can't help his hair because it is curly or maybe because he isn't smiling. I don't know...but I didn't like the beard or the mustache, just as he reminds me of the Letter $S$ in the girls. He looks like he could be sullen.

Middle-Aged Stimulus Photographs:

Most Physically Attractive

LETTER W: The expression, obviously somebody who enjoys life. If that is projected in someway physically...he's not unkept--but just from brief appearance not someone who is overly concerned about how they look. He's not as I said, I know I tend to be especially... on first appearances..prejudice of people--men.. who are overweight. However, one of the nicest persons I know and I have good feelings about on a friendship level is extremely overweight but he has so much going for him that compensates.

Least Physically Attractive

LETTER E: The four men I considered to be overweight here again I'm.... he has a short cropped hair cut--sort of military mentality. I'm projecting someone who again might represent a conservative point of view, looks like he has kind of a pantsuit on...kind of an overweight effect. Its not the mustache---because the last few men I have dated.... He's conservative, salesman type person. I tend to go for the casual look. 
Old Stimulus Photographs:

Most Physically Attractive

LETTER BBB: He has a nice smile, he looks like he is kind of self-assured and...he looks like he has weathered a few storms but he has come out with a positive attitude in life. He looks like he would be a friendly fellow, very congenial and he was probably a very handsome man when he was younger, but he is still an attractive man.

Least Physically Attractive

LETTER HH: He just has got a firmness set in his jaw or something and something in his eye that sort of..... is kind of cold to me, like he might be a little hard to warm up to... that may not be the case at all but something about his jaw is.... That sounds crazy. 
Selection Judges Tape-recorded Comments on their Definition of Physical Attractiveness on Each Age Group's Gender.

Young Male Stimulus Photographs:

What is your Definition of Physical Attractiveness?

Well, um.... I think there are two elements of which I have been saying ....one has to do with the overall impression that the appearance gives and I'm attributing some personal characteristics or attitude based on appearance to some degree. Then, on the other hand, strictly speaking the warm, the graphic quality of the pictures so that I would have to say things like skin, tone, color, or the balance of the features and that sort. I guess... I am responding to both senses to some degree too... kind of posture and I am sure you have tried to control to get the same pictures but there are some slight difference in which I am responding to that. I am responding to these as partly photographs then partly making attributions of what the people would be like based on the photographs. I think objectively speaking this $M$ is a better photograph just in terms of composition, color and so forth then the other three. Partly the formal ity maybe a little off kind of a fluorescent green to that picture.... the background is off agan....it is the photograph.

\section{What is Your Definition of Physical Attractiveness?}

I think Physical attractiveness is an attitude, more than anything else. A person that is unattractive.... a person he/she does certain things to their appearance and their personality and with their attitude they can decrease the unattractiveness by doing something with their hair differently, maybe losing a little bit of weight, dressing differently. Your attitude has alot to do with your outside appearance. I have seen people who have frowned for 25 years and they have got lines in their face, where they have a permanent frown. Her attitude has effected her physical attractiveness over a period of time. That is my definition of physical attractiveness.

Middle-Aged Male Stimulus Photographs:

What is your Definition of Physical Attractiveness?

In general, first of all, the way they are dressed is important to me, the way the hair is styled and generally a smile is important to me and if their face is showing something--like smiling where you can see like in Letter P picture you don't know if he is kind of smiling or if he's really afraid of something, scared--you can't tell what he is thinking. Like the CC picture, she is smiling and showing happiness.

What is your definition of physical attractiveness?

Those people that find physical attractiveness... I really can't define physical attractiveness. Physical attractiveness is a variable for different kinds of persons and different kinds of contexts.... attributes become precursors of physical attractiveness. 
Middle-Aged Female Stimulus Photographs:

What is your Definition of Physical Attractiveness?

I can recall vague recognitions or people I have identified with both the most and least physically attractive.

What is your Definition of Physical Attractiveness?

A person that is trying to be relational and not contrast with such...as a person with a very strong ego, rather than coming from the mind from the heart in relationships with people and trying to relate in that way-heart felt relationships rather than mental relationships--mentalizing. And that's basically it.

Old Male Stimulus Photographs:

What is your Definition of Physical Attractiveness?

Someone who looks the part, physically alert... By there profile, there look, eyes, nose, mouth--whether they are alert and the nose is in the middle of their face.. whether their mouth is a happy mouth.

What is your Definition of Physical Attractiveness?

Age is a matter of self concept. If you were to ask me how old I was I would say between the age of 30 and 35 however, I may have acquired more knocks, but I had learned the most within that age span. Aging is societal.. When you say you are 30 you are considered over the hill and down. I think a child equates aging with height. I have seen some 30 year olds who act 65 and should be retired at 30 . As I had said, I think aging is societal where it completes a conflict because of the successfuliy.........according to our society, a kind being 21-22, has got to be a success out of necessity. Anybody occuping a place where he is, or sees himself obviously becomes a threat and an enemy but of course it works the other way. You have this big fat juicy conflict. Old Female Stimulus Photographs:

What is your Definition of Physical Attractiveness?

They have to look alive or what I call it--sparkle and healthy. I don't like too much hair or too long. I remembered the time when everything was trim and maybe I'm thinking a little like that.

What is your Definition of Physical Attractiveness?

Person has to have a natural look about them.....to me... If...for instance... I'm not attracted to a bowl hair cut or his hair shaved off, sideburns not to the extreme is attractive but it depends on the type if a man is sort of a Latin type... I think they look especially good and also mustaches like down in Mexico... they give them a romantic look... 
they wouldn't have otherwise. For men I don't like their hair do's to be too sharp to look like they came out of a beauty shop...spraying them and doing this or that. I like a man to look like a real man. A man that could go outdoors, go fishing or ride a horse--that's the type of man I would be attracted to.

What is your Definition of Physical Attractiveness?

Physical attractiveness is a sense of humor, neatness, not being overly obese or underweight but having a trim figure..but being mentally alert and vivacious. 


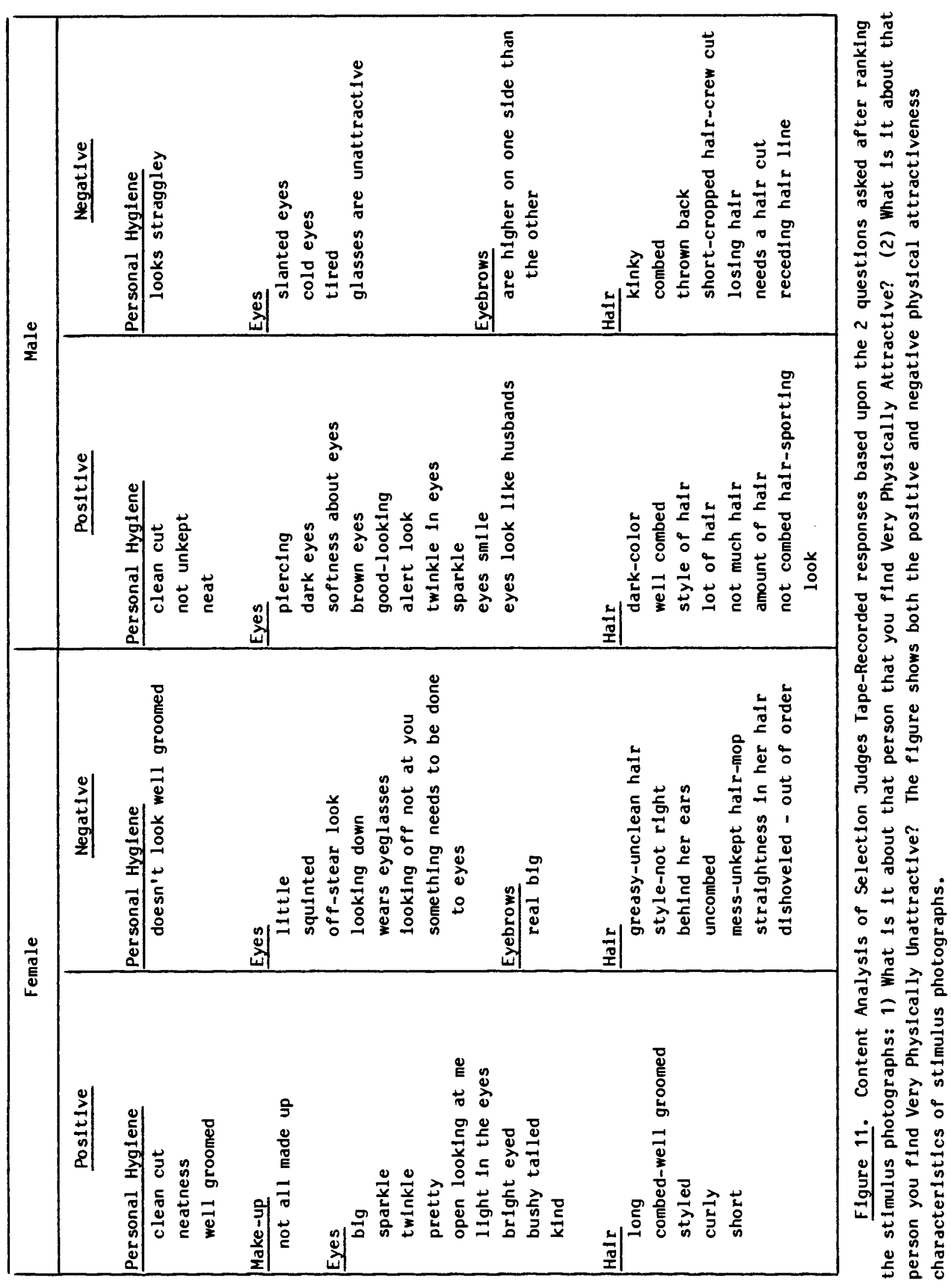




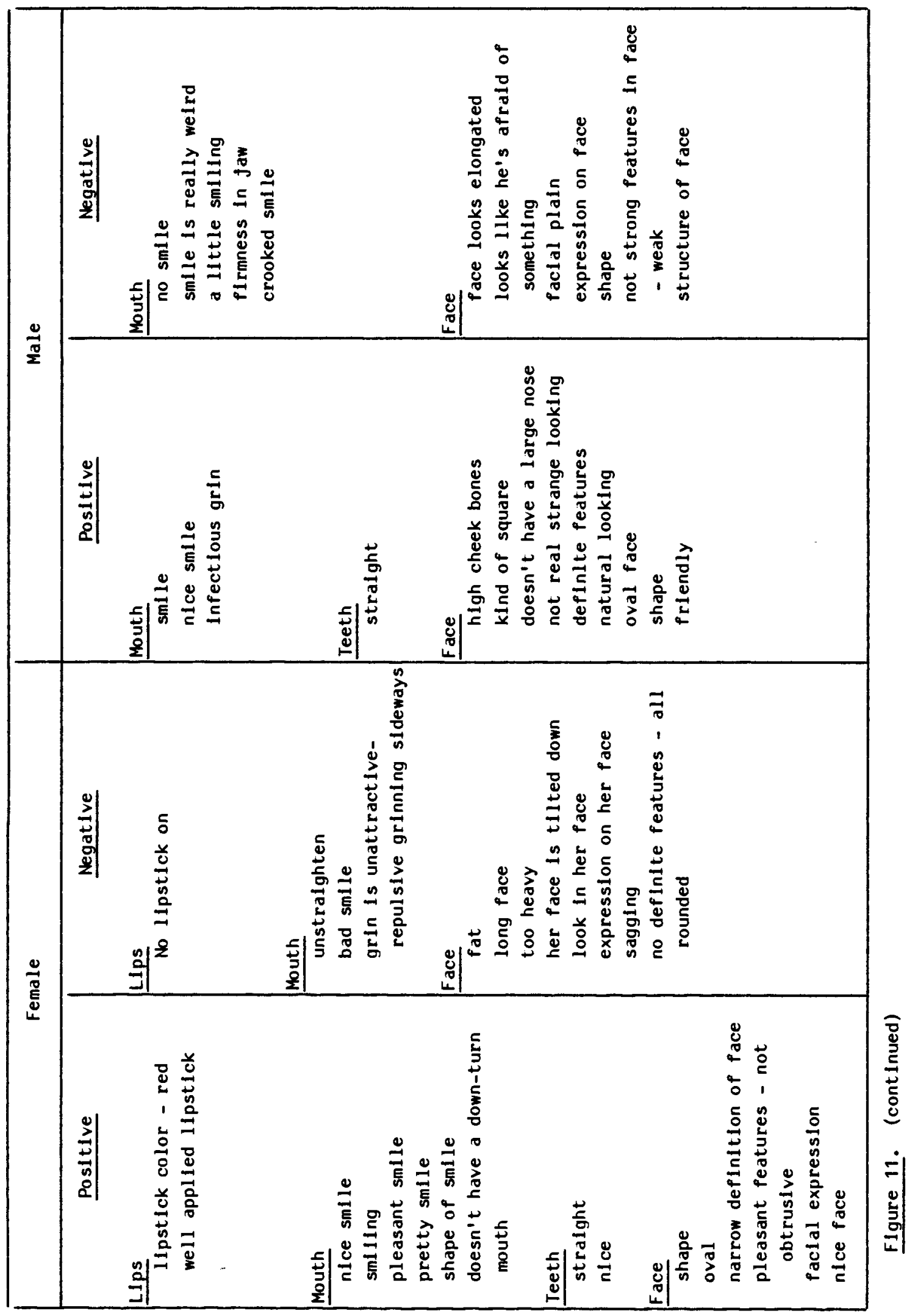




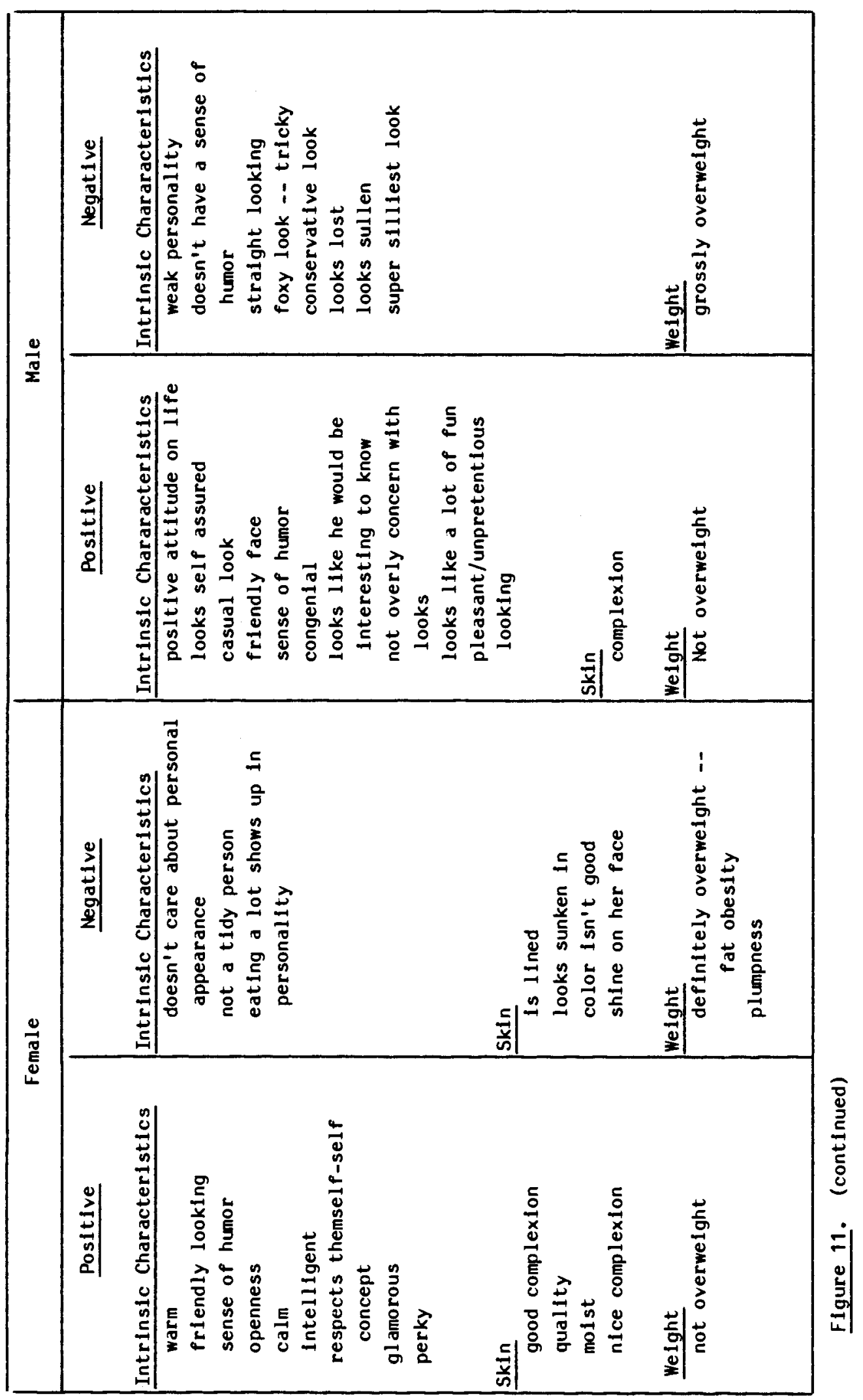




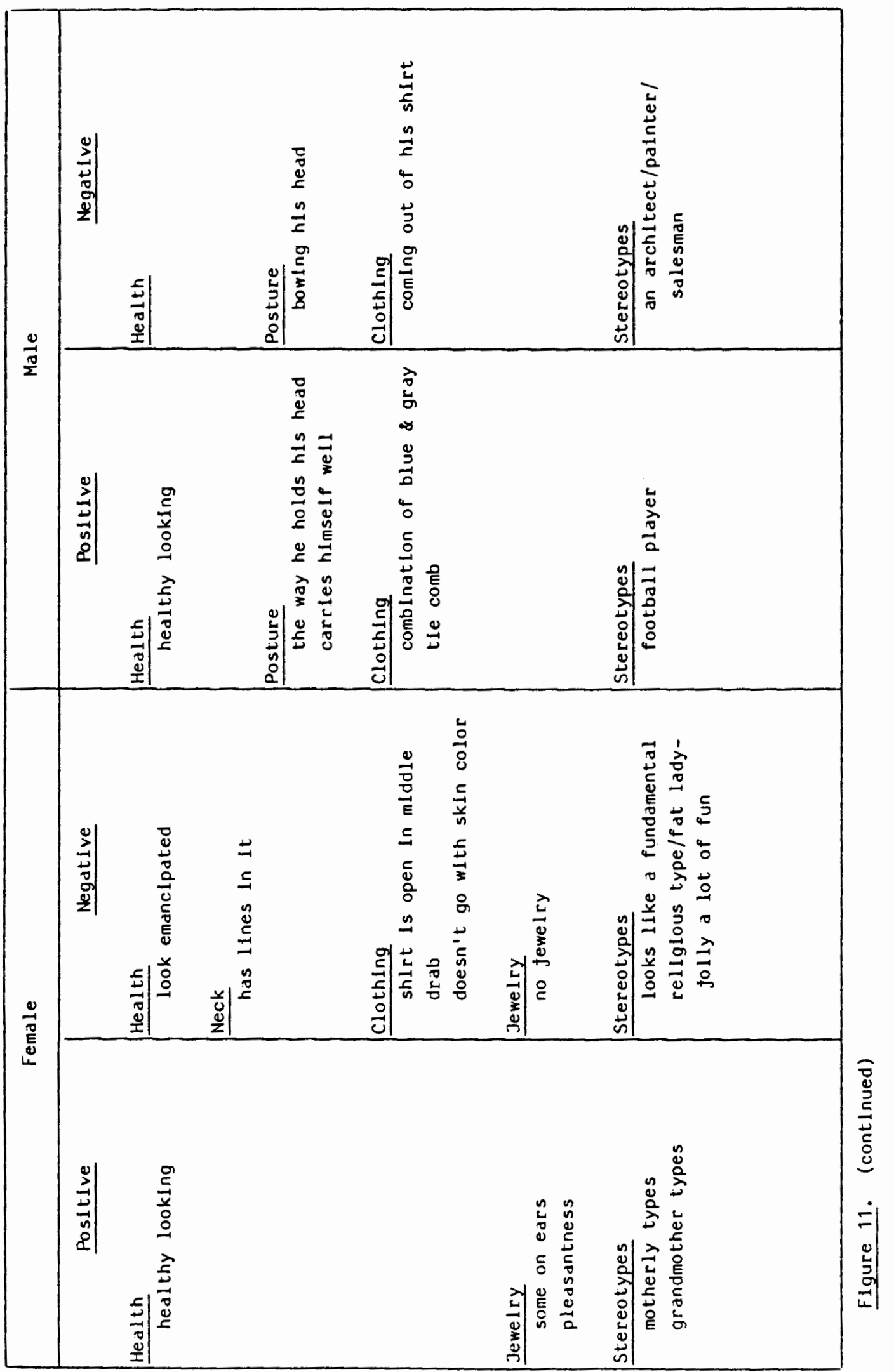


TABLE XXXIII

DESCRIPTIVE STATISTICS OF YOUNG, MIDDLE-AGED, OLD SELECTION JUDGES RANKING/RATING YOUNG STIMULUS PHOTOGRAPHS

PHYSICAL ATTRACTIVENESS

\begin{tabular}{|c|c|c|c|c|}
\hline Photo ID & Mean & $\begin{array}{l}\frac{\text { ngs }}{\text { Standard }} \\
\text { Deviation }\end{array}$ & Mean & $\begin{array}{l}\text { ings } \\
\text { Standard } \\
\text { Deviation }\end{array}$ \\
\hline
\end{tabular}

\begin{tabular}{|c|c|c|c|c|}
\hline \multicolumn{5}{|c|}{ Young Male Stimuli } \\
\hline $\begin{array}{r}D \\
N \\
\star J \\
Q \\
\star T \\
L \\
B \\
\star R\end{array}$ & $\begin{array}{l}3.17 \\
4.00 \\
4.17 \\
5.50 \\
3.17 \\
5.17 \\
5.00 \\
5.83\end{array}$ & $\begin{array}{l}2.79 \\
2.68 \\
2.04 \\
1.76 \\
1.83 \\
2.48 \\
2.68 \\
1.60\end{array}$ & $\begin{array}{l}4.50 \\
4.50 \\
4.30 \\
5.17 \\
4.00 \\
5.17 \\
5.17 \\
4.67\end{array}$ & $\begin{array}{r}1.76 \\
1.22 \\
1.03 \\
1.47 \\
1.26 \\
.75 \\
1.17 \\
1.03\end{array}$ \\
\hline \multicolumn{5}{|c|}{ Young Female Stimuli } \\
\hline $\begin{array}{c}G \\
K \\
\star M \\
\star 0 \\
\star F \\
A A \\
E \\
S\end{array}$ & $\begin{array}{l}2.00 \\
2.33 \\
2.17 \\
4.50 \\
4.67 \\
6.33 \\
6.17 \\
7.83\end{array}$ & $\begin{array}{l}1.79 \\
1.03 \\
1.21 \\
1.38 \\
1.21 \\
1.21 \\
1.17 \\
2.36\end{array}$ & $\begin{array}{l}2.50 \\
2.50 \\
3.33 \\
4.83 \\
3.70 \\
4.50 \\
4.83 \\
5.50\end{array}$ & $\begin{array}{r}1.05 \\
1.37 \\
1.63 \\
1.47 \\
1.63 \\
1.64 \\
.75 \\
1.16\end{array}$ \\
\hline
\end{tabular}

Number of observations, $\mathrm{N}=8$

*The asterisk symbol indicates the selected stimuli used throughout the study.

Note: Each mean and standard deviation was rounded off to the nearest hundredth. 
TABLE XXXIV

DESCRIPTIVE STATISTICS OF YOUNG, MIDDLE-AGED, OLD SELECTION JUDGES RANKING/RATING MIDDLE-AGED STIMULUS PHOTOGRAPHS

PHYSICAL ATTRACTIVENESS

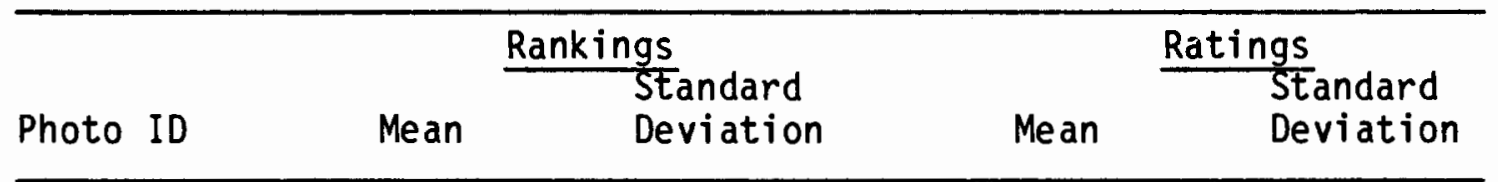

\begin{tabular}{|c|c|c|c|c|}
\hline \multirow[b]{2}{*}{$\begin{array}{c}X \\
W \\
P \\
V \\
\star * F F \\
\star Z \\
\star U \\
Y\end{array}$} & \multicolumn{3}{|c|}{ Middle-age Male Stimuli } & \multirow[b]{2}{*}{$\begin{array}{r}.41 \\
.75 \\
1.17 \\
1.37 \\
1.38 \\
1.79 \\
1.03 \\
1.67\end{array}$} \\
\hline & $\begin{array}{l}3.50 \\
1.67 \\
5.50 \\
5.00 \\
4.83 \\
5.83 \\
5.67 \\
5.33\end{array}$ & $\begin{array}{r}1.17 \\
.82 \\
2.43 \\
1.10 \\
2.79 \\
4.16 \\
1.75 \\
2.34\end{array}$ & $\begin{array}{l}3.17 \\
2.83 \\
3.83 \\
3.67 \\
4.50 \\
4.00 \\
3.67 \\
4.00\end{array}$ & \\
\hline \multicolumn{5}{|c|}{ Middle-age Female Stimuli } \\
\hline $\begin{array}{c}C \\
\star E E \\
\star C C \\
H \\
A \\
\text { BB } \\
\text { DD } \\
\star I\end{array}$ & $\begin{array}{l}3.83 \\
3.00 \\
4.33 \\
3.33 \\
3.83 \\
3.83 \\
7.50 \\
6.33\end{array}$ & $\begin{array}{l}1.94 \\
1.79 \\
2.16 \\
2.16 \\
1.72 \\
2.23 \\
.55 \\
2.25\end{array}$ & $\begin{array}{l}3.33 \\
3.17 \\
3.50 \\
3.67 \\
3.67 \\
3.67 \\
3.67 \\
3.67\end{array}$ & $\begin{array}{r}1.21 \\
1.17 \\
.84 \\
1.03 \\
.82 \\
1.21 \\
1.63 \\
1.63\end{array}$ \\
\hline
\end{tabular}

Number of observations, $\mathrm{N}=8$

*The asterisk symbol indicates the selected stimuli used throughout the study.

Note: Each mean and standard deviation was rounded off to the nearest hundredth. 
TABLE XXXV

DESCRIPTIVE STATAISTICS OF YOUNG, MIDDLE-AGED, OLD SELECTION JUDGES RANKING/RATING OLD STIMULUS PHOTOGRAPHS

PHYSICAL ATTRACTIVENESS

\begin{tabular}{llll}
\hline & Rankings & Ratings \\
\cline { 3 - 4 } & Mhoto ID & Meandard \\
Deviation & Mean & \begin{tabular}{l} 
Deviation \\
\hline
\end{tabular}
\end{tabular}

01d Male Stimuli

$\begin{array}{ccccc}\star 00 & 7.83 & .41 & 4.83 & .98 \\ \text { AAA } & 2.67 & 1.86 & 4,16 & 1.17 \\ \text { BBB } & 2.83 & 1.83 & 3.83 & 1.33 \\ \star Y Y & 3.33 & 2.25 & 4.00 & 1.79 \\ \text { LL } & 6.33 & .82 & 4.50 & 1.38 \\ \text { HH } & 5.17 & 2.32 & 3.83 & 2.41 \\ \star P P & 3.50 & 1.52 & 3.67 & 1.63 \\ Z Z & 4.33 & 1.37 & 3.83 & 1.17\end{array}$

0ld Female Stimuli

\begin{tabular}{|c|c|c|c|c|}
\hline $\begin{array}{l}\text { *MM } \\
\text { *KK } \\
\text { WW } \\
\text { NN } \\
\text { *I I } \\
\text { TT } \\
\text { VV } \\
\text { UU }\end{array}$ & $\begin{array}{l}3.00 \\
3.00 \\
2.83 \\
4.83 \\
4.67 \\
7.67 \\
2.67 \\
7.33\end{array}$ & $\begin{array}{r}1.41 \\
1.90 \\
1.47 \\
1.47 \\
1.63 \\
.52 \\
1.63 \\
.52\end{array}$ & $\begin{array}{l}3.83 \\
3.33 \\
4.00 \\
4.33 \\
4.83 \\
5.83 \\
3.67 \\
5.83\end{array}$ & $\begin{array}{r}.75 \\
.52 \\
1.53 \\
1.21 \\
1.33 \\
1.60 \\
1.03 \\
.98\end{array}$ \\
\hline
\end{tabular}

Number of observations $\mathrm{N}=8$

*The asterisk symbol indicates the selected stimuli used throughout the study.

Note: Each mean and standard deviation was rounded off to the nearest hundredth. 
TABLE XXXVI

DESCRIPTIVE STATISTICS OF YOUNG MIDDLE-AGED, OLD MALE SELECTION JUDGES RANKING/RATING YOUNG STIMULUS PHOTOGRAPHS

PHYSICAL ATTRACTIVENESS

\begin{tabular}{|c|c|c|c|c|}
\hline Photo ID & Mean & $\begin{array}{l}\text { ngs } \\
\text { Standard } \\
\text { Deviation }\end{array}$ & Mean & $\begin{array}{l}\text { Stgs } \\
\text { Standard } \\
\text { Deviation }\end{array}$ \\
\hline
\end{tabular}

Young Male Stimuli

$\begin{array}{rrrrr}D & 1.67 & .58 & 3.67 & 1.15 \\ N & 6.00 & 1.73 & 5.00 & 1.73 \\ \star J & 5.67 & 1.53 & 5.00 & 1.00 \\ Q & 4.00 & 2.44 & 4.33 & 1.53 \\ \star T & 2.00 & 1.73 & 3.33 & 1.53 \\ \star \mathrm{L} & 5.67 & 2.31 & 5.00 & 1.00 \\ \mathrm{~B} & 4.33 & 3.21 & 4.67 & 1.53 \\ \mathrm{R} & 6.67 & 1.15 & 4.67 & .58\end{array}$

Young Female Stimuli

$\begin{array}{crrrr}G & 2.33 & 1.15 & 2.33 & 1.53 \\ K & 2.70 & 1.15 & 2.33 & 1.53 \\ \star M & 1.70 & 1.15 & 2.00 & 1.00 \\ 0 & 4.10 & 2.08 & 4.67 & 1.73 \\ \star F & 4.30 & .58 & 3.00 & 1.00 \\ A A & 6.00 & 1.00 & 3.67 & 1.53 \\ E & 6.67 & .58 & 4.67 & .58 \\ S & 8.00 & 0 & 5.33 & 1.53\end{array}$

Number of observations $\mathrm{N}=8$

*The asterisk symbol indicates the selected stimuli used throughout the study.

Note: Each mean and standard deviation was rounded off to the nearest hundredth. 
TABLE XXXVII

DESCRIPTIVE STATISTICS OF YOUNG, MIDDLE-AGED, OLD MALE SELECTION JUDGES RANKING/RATING MIDDLE-AGED STIMULUS PHOTOGRAPHS

PHYSICAL ATTRACTIVENESS

\begin{tabular}{llll}
\hline & Rankings & Ratings \\
\cline { 3 - 4 } & Meandard & $\begin{array}{l}\text { Standard } \\
\text { Deviation }\end{array}$ \\
\hline
\end{tabular}

Middle-age Male Stimuli

$\begin{array}{lllll}X & 2.33 & 1.53 & 3.33 & .58 \\ W & 2.00 & 1.00 & 3.00 & 1.00 \\ P & 7.00 & 1.00 & 4.00 & 1.00 \\ V & 5.33 & 1.53 & 3.00 & 1.73 \\ \star F F & 4.00 & 3.61 & 4.33 & .58 \\ \star Z & 5.67 & 1.53 & 3.67 & 2.31 \\ \star U & 6.00 & 1.73 & 3.33 & 1.15 \\ Y & 3.70 & 2.08 & 3.00 & 1.00\end{array}$

Middle-age Female Stimuli

\begin{tabular}{crrrr}
$C$ & 5.00 & 1.73 & 3.67 & 1.53 \\
*EE & 4.33 & 1.53 & 3.33 & 1.53 \\
*CC & 3.33 & 2.08 & 3.67 & .58 \\
H & 2.67 & 1.15 & 3.67 & .58 \\
A & 3.33 & 1.53 & 4.00 & 0 \\
BB & 2.33 & 2.31 & 3.67 & 1.15 \\
DD & 7.33 & .58 & 3.00 & 1.73 \\
*I & 7.67 & .58 & 3.67 & 1.53 \\
\hline
\end{tabular}

Number of observations $\mathrm{N}=8$

*The asterisk symbol indicates the selected stimuli used throughout the study.

Note: Each mean and standard deviation was rounded off to the nearest hundredth. 
TABLE XXXVIII

DESCRIPTIVE STATISTICS OF YOUNG, MIDDLE-AGED, OLD MALE SELECTION JUDGES RANKING/RATING OLD STIMULUS PHOTOGRAPHS

PHYSICAL ATTRACTIVENESS

\begin{tabular}{|c|c|c|c|c|}
\hline Photo ID & Mean & $\begin{array}{l}\text { kings } \\
\text { Standard } \\
\text { Deviation }\end{array}$ & Mean & $\begin{array}{l}\frac{\text { ngs }}{\text { Standard }} \\
\text { Deviation }\end{array}$ \\
\hline \multicolumn{5}{|c|}{ Old Male Stimuli } \\
\hline $\begin{array}{l}\star 00 \\
\text { AAA } \\
\text { BBB } \\
\star Y Y \\
\text { LL } \\
\text { HH } \\
\star P P \\
Z Z\end{array}$ & $\begin{array}{l}8.00 \\
1.67 \\
3.33 \\
3.67 \\
6.33 \\
3.67 \\
4.33 \\
5.00\end{array}$ & $\begin{array}{r}0 \\
.73 \\
.97 \\
.37 \\
.37 \\
1.4 \\
.97 \\
1.10\end{array}$ & $\begin{array}{l}4.67 \\
4.00 \\
4.67 \\
4.67 \\
5.00 \\
4.00 \\
4.67 \\
4.67\end{array}$ & $\begin{array}{r}.73 \\
1.10 \\
.73 \\
.37 \\
.63 \\
0 \\
.73 \\
.37\end{array}$ \\
\hline \multicolumn{5}{|c|}{ Old Female Stimuli } \\
\hline $\begin{array}{l}\star M M \\
\star K K \\
W W \\
W N \\
\star I I \\
\text { TT } \\
\text { VV } \\
\text { UU }\end{array}$ & $\begin{array}{l}3.00 \\
4.33 \\
2.33 \\
5.33 \\
4.67 \\
7.33 \\
1.33 \\
7.67\end{array}$ & $\begin{array}{l}1.10 \\
.97 \\
.37 \\
.37 \\
.73 \\
.37 \\
.37 \\
.37\end{array}$ & $\begin{array}{l}4.00 \\
3.67 \\
4.33 \\
4.67 \\
5.00 \\
6.00 \\
4.33 \\
6.00\end{array}$ & $\begin{array}{r}.63 \\
.37 \\
.37 \\
.37 \\
1.10 \\
.63 \\
.37 \\
0\end{array}$ \\
\hline
\end{tabular}

Number of observations $\mathrm{N}=8$

*The asterisk symbol indicates the selected stimuli used throughout the study.

Note: Each mean and standard deviation was rounded off to the nearest hundredth. 
TABLE XXXIX

DESCRIPTIVE STATISTICS OF YOUNG, MIDDLE-AGED, OLD FEMALE SELECTION JUDGES RANKING/RATING YOUNG STIMULUS PHOTOGRAPHS

PHYSICAL ATTRACTIVENESS

\begin{tabular}{|c|c|c|c|c|}
\hline \multirow[b]{2}{*}{ Photo ID } & \multicolumn{2}{|c|}{ Rankings } & \multicolumn{2}{|c|}{ Ratings } \\
\hline & Mean & $\begin{array}{l}\text { Standard } \\
\text { Deviation }\end{array}$ & Mean & $\begin{array}{l}\text { Standard } \\
\text { Deviation }\end{array}$ \\
\hline
\end{tabular}

Young Male Stimuli

$\begin{array}{rrrrr}L & 4.70 & 3.51 & 5.33 & .58 \\ B & 5.70 & 1.15 & 5.67 & .58 \\ \star T & 4.33 & 2.52 & 4.67 & .58 \\ Q & 7.00 & 0 & 6.00 & 1.00 \\ \star R & 5.00 & 1.73 & 4.67 & 1.53 \\ \star \mathrm{J} & 2.70 & 1.15 & 3.67 & .58 \\ D & 4.70 & 3.51 & 5.33 & 2.08 \\ \mathrm{~N} & 2.00 & 1.73 & 4.00 & 0\end{array}$

Young Female Stimuli

\begin{tabular}{rrrrr}
$G$ & 1.67 & .37 & 2.67 & .58 \\
$K$ & 2.00 & 1.65 & 2.67 & 1.53 \\
$\star M$ & 2.70 & .97 & 4.67 & .58 \\
$E$ & 5.70 & .96 & 2.50 & 1.00 \\
$\star 0$ & 4.70 & .82 & 5.67 & .58 \\
$\star F$ & 5.00 & 1.10 & 4.33 & 2.08 \\
$A A$ & 6.70 & .96 & 5.33 & 1.53 \\
$S$ & 7.70 & .37 & 6.33 & .58 \\
\hline
\end{tabular}

Number of observations $\mathrm{N}=8$

*The asterisk symbol indicates the selected stimuli used throughout the study.

Note: Each mean and standard deviation was rounded off to the nearest hundredth. 
TABLE XL

DESCRIPTIVE STATISTICS OF YOUNG, MIDDLE-AGED, OLD FEMALE SELECTION JUDGES RANKING/RATING MIDDLE-AGED STIMULUS PHOTOGRAPHS

PHYSICAL ATTRACTIVENESS

\begin{tabular}{llll}
\hline & Rankings & & Ratings \\
\cline { 3 - 4 } & $\begin{array}{l}\text { Standard } \\
\text { Peviation }\end{array}$ & Mean & $\begin{array}{r}\text { Stand } \\
\text { Deviation }\end{array}$ \\
\hline
\end{tabular}

Middle-age Male Stimuli

$\begin{array}{ccccc}X & 2.00 & 1.00 & 3.00 & 0 \\ W & 1.33 & .58 & 2.67 & .58 \\ P & 4.00 & 2.65 & 3.67 & 1.53 \\ V & 4.67 & .58 & 4.33 & .58 \\ \star F F & 5.67 & 2.08 & 4.67 & 2.08 \\ & & & & \\ \star Z & 6.00 & 2.00 & 4.33 & 1.53 \\ \star U & 5.33 & 2.08 & 4.00 & 1.00 \\ Y & 7.00 & 1.00 & 5.00 & 1.73\end{array}$

Middle-age Female Stimuli

$\begin{array}{crrrr}C & 2.67 & 1.53 & 3.00 & 1.41 \\ \star \mathrm{EE} & 2.00 & .58 & 3.00 & 1.41 \\ { }^{\mathrm{CC}} & 5.33 & 2.08 & 3.33 & 1.15 \\ \mathrm{H} & 4.00 & 3.00 & 3.67 & 1.53 \\ \mathrm{~A} & 4.33 & 2.08 & 3.33 & 1.15 \\ \mathrm{BB} & 5.33 & .58 & 3.67 & 1.53 \\ \mathrm{DD} & 7.67 & .58 & 4.33 & 1.53 \\ \text { *I } & 5.00 & 2.65 & 3.67 & 2.08\end{array}$

Number of observations $\mathrm{N}=8$

*The asterisk symbol indicates the selected stimuli used throughout the study.

Note: Each mean and standard deviation was rounded off to the nearest hundredth. 
TABLE XLI

DESCRIPTIVE STATISTICS OF YOUNG, MIDDLE-AGED, OLD FEMALE SELECTION JUDGES RANKING/RATING OLD STIMULUS PHOTOGRAPHS

PHYSICAL ATTRACTIVENESS

\begin{tabular}{llll}
\hline & Rankings & Ratings \\
\cline { 3 - 4 } & Mhoto ID & Standard \\
Deviation & Mean & $\begin{array}{l}\text { Standard } \\
\text { Deviation }\end{array}$ \\
\hline
\end{tabular}

0ld Male Stimul $\mathbf{i}$

$\begin{array}{ccccr}\star 00 & 7.67 & .37 & 5.00 & .63 \\ \text { AAA } & 3.67 & 1.32 & 4.33 & .37 \\ \text { BBB } & 2.33 & 1.46 & 3.00 & .63 \\ \star Y Y & 3.00 & 1.26 & 3.33 & 1.59 \\ \text { LL } & 6.33 & .73 & 4.00 & 1.10 \\ \text { HH } & 6.67 & .73 & 5.00 & 0 \\ \star P P & 2.67 & .003 & 2.67 & .97 \\ \text { ZZ } & 6.00 & .37 & 3.00 & .63\end{array}$

01d Female Stimuli

\begin{tabular}{rrrrr}
$\star M M$ & 2.67 & .63 & 3.67 & .37 \\
$\star$ KKK & 1.67 & .73 & 3.00 & 0 \\
WW & 3.33 & 1.32 & 3.67 & 1.46 \\
NN & 4.33 & 1.32 & 4.00 & 1.10 \\
$\star I I$ & 4.67 & 1.46 & 4.67 & 3.16 \\
TT & 8.00 & 0 & 5.67 & .01 \\
VV & 4.00 & .63 & 3.00 & .63 \\
UU & 7.00 & 0 & 5.67 & .97 \\
\hline
\end{tabular}

Number of observations $\mathrm{N}=8$

*The asterisk symbol indicates the selected stimuli used throughout the study.

Note: Each mean and standard deviation was rounded off to the nearest hundredth. 
TABLE XLII

GROUP MEAN, STANDARD DEVIATION AND RANGE OF YOUNG, MIDDLE-AGED, OLD SELECTION JUDGES RATING MALE AND FEMALE YOUNG, MIDDLE-AGED

AND OLD STIMULUS PHOTOGRAPHS'

\begin{tabular}{llll}
\hline $\begin{array}{l}\text { Gender of Stimuli } \\
\text { Age Group }\end{array}$ & $\begin{array}{l}\text { Group } \\
\text { Mean }\end{array}$ & $\begin{array}{l}\text { Standard } \\
\text { Deviation }\end{array}$ & Range \\
\hline
\end{tabular}

Male

$\begin{array}{llll}\text { Young } & 4.59 & 1.38 & 3-5 \\ \text { Middle-Aged } & 3.63 & 1.36 & 2-5 \\ \text { Old } & 4.08 & 1.38 & 2-5\end{array}$

Female

$\begin{array}{llll}\text { Young } & 3.91 & 1.77 & 3-7 \\ \text { Middle-Aged } & 3.46 & 1.24 & 2-4 \\ 01 d & 4.36 & 1.53 & 3-6\end{array}$

$\underline{\text { Total * }}$

$\begin{array}{llll}\text { Young } & 4.29 & 1.56 & 3-7 \\ \text { Middle-Aged } & 3.58 & 1.25 & 2-5 \\ \text { 01d } & 4.26 & 1.40 & 2-6\end{array}$

Number of observations, $N=48$

* Number of total observations, $N=96$ (combination of both Male \& Female Stimuli within each age group). 
TABLE XLIII

GROUP MEAN, STANDARD DEVIATION AND RANGE OF YOUNG, MIDDLE-AGED, OLD MALE SELECTION JUDGES RATING MALE AND FEMALE YOUNG, MIDDLE-AGED AND OLD STIMULUS PHOTOGRAPHS

\begin{tabular}{llll}
\hline $\begin{array}{l}\text { Gender of Stimuli } \\
\text { Age Group }\end{array}$ & $\begin{array}{l}\text { Group } \\
\text { Mean }\end{array}$ & $\begin{array}{l}\text { Standard } \\
\text { Deviation }\end{array}$ & Range \\
\hline $\begin{array}{l}\text { Male } \\
\text { Young }\end{array}$ & 4.28 & 1.51 & $3-4$ \\
Middle-Aged & 3.32 & 1.34 & $2-4$ \\
Old & 4.36 & 1.28 & $2-4$ \\
Female & & & \\
Young & 3.28 & 1.72 & $3-5$ \\
Middle-Aged & 3.44 & 1.26 & $3-4$ \\
Old & 4.56 & 1.44 & $3-5$ \\
\hline
\end{tabular}

Number of observations, $N=24$ 


\section{TABLE XLIY}

GROUP MEAN, STANDARD DEVIATION AND RANGE OF YOUNG, MIDDLE-AGED, OLD FEMALE SELECTION JUDGES RATING MALE AND FEMALE YOUNG, MIDOLE-AGED AND OLD STIMULUS PHOTOGRAPHS

\begin{tabular}{llll}
\hline $\begin{array}{l}\text { Gender of Stimuli } \\
\text { Age Group }\end{array}$ & $\begin{array}{l}\text { Group } \\
\text { Mean }\end{array}$ & $\begin{array}{l}\text { Standard } \\
\text { Deviation }\end{array}$ & Range \\
\hline $\begin{array}{l}\text { Male } \\
\text { Young }\end{array}$ & 4.72 & 1.51 & $4-5$ \\
Middle-Aged & 3.80 & 1.52 & $2-5$ \\
O1d & 3.64 & 1.60 & $3-5$ \\
Female & & & \\
Young & 4.40 & 1.82 & $4-7$ \\
Middle-Aged & 3.36 & 1.41 & $2-3$ \\
01d & 4.00 & 1.80 & $4-6$ \\
\hline
\end{tabular}

Number of observations, $N=24$ 
APPENDIX H

STIMULUS PHOTOGRAPHS 


\section{YOUNG STIMULUS PHOTOGRAPHS}
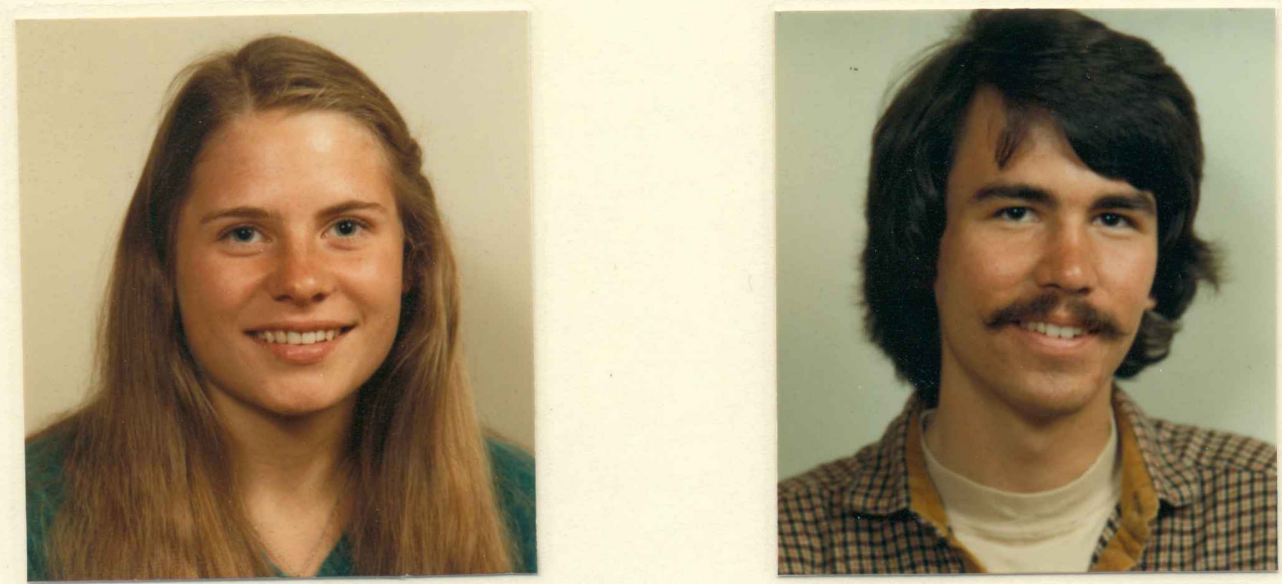

HIGH PHYSICAL ATTRACTIVENESS INTENSITY LEVEL
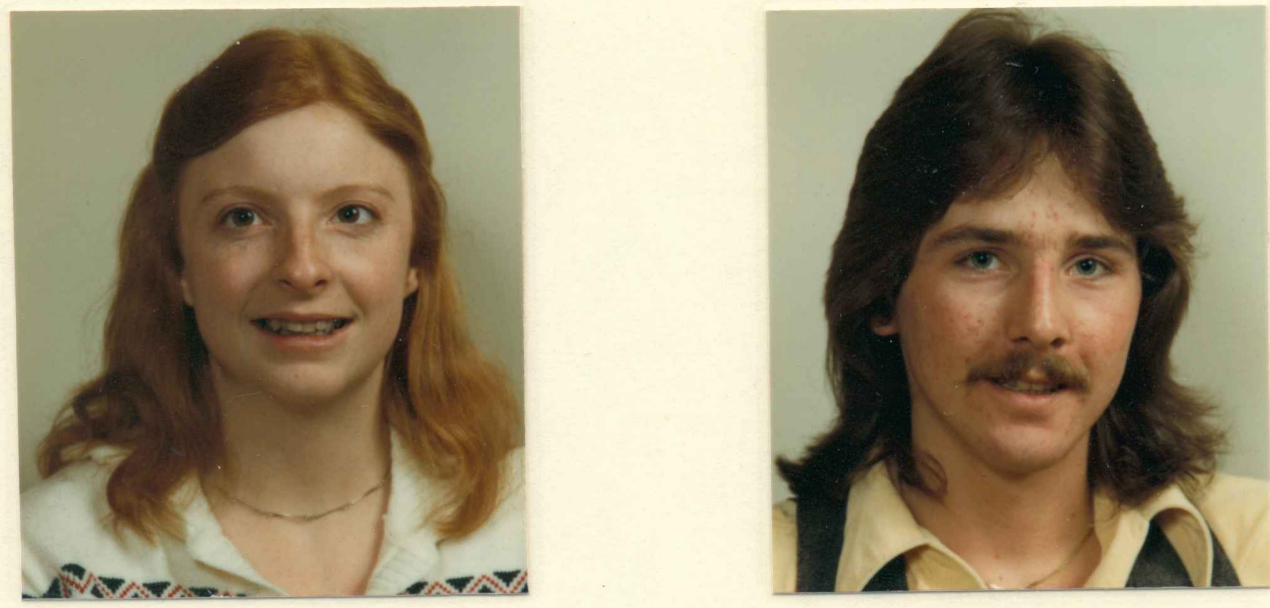

MEDIUM PHYSICAL ATTRACTIVENESS INTENSITY LEVEL
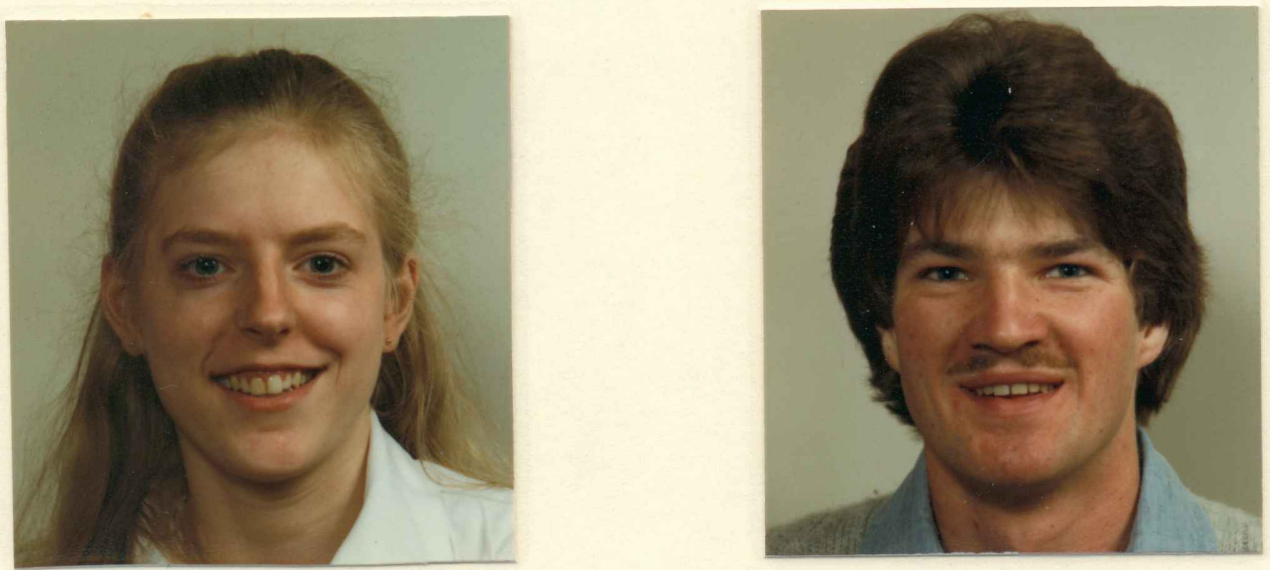

LOW PHYSICAL ATTRACTIVENESS INTENSITY LEVEL 


\section{MIDDLE-AGED STIMULUS PHOTOGRAPHS}
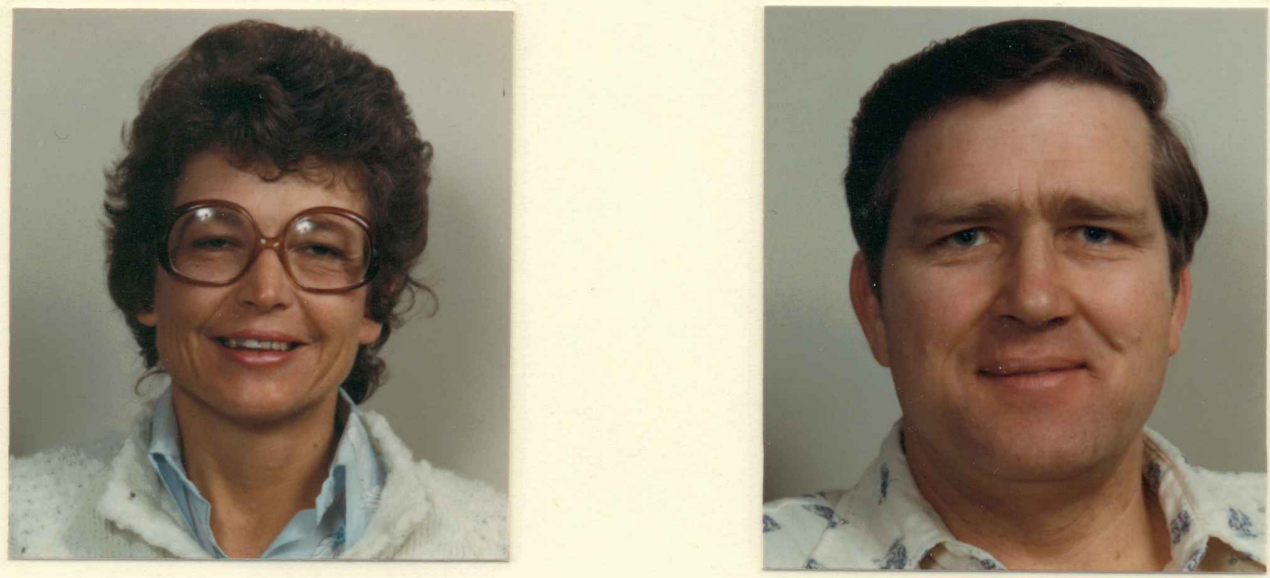

HIGH PHYSICAL ATTRACTIVENESS INTENSITY LEVEL
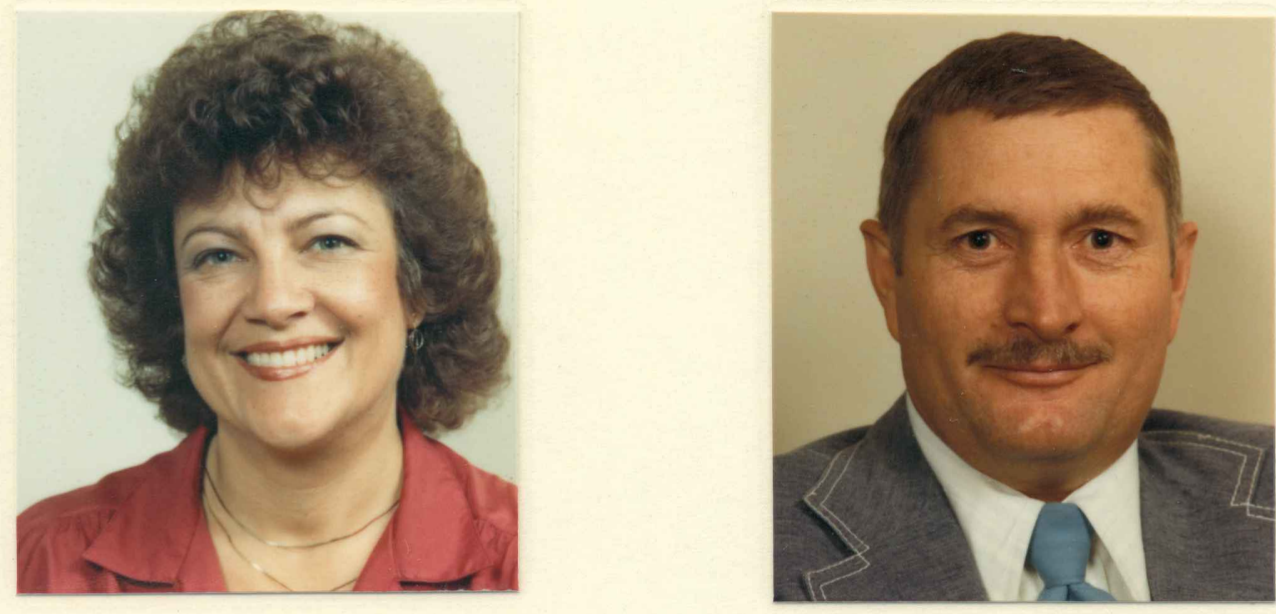

MEDIUM PHYSICAL ATTRACTIVENESS INTENSITY LEVEL
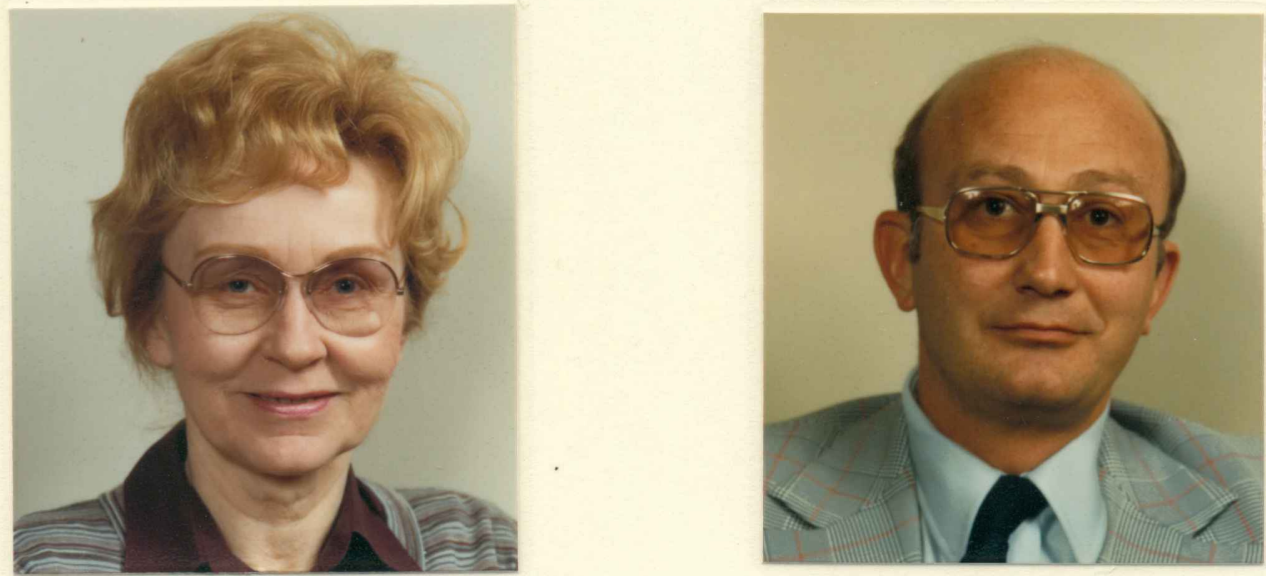

LOW PHYSICAL ATTRACTIVENESS INTENSITY LEVEL 


\section{OLD STIMULUS PHOTOGRAPHS}
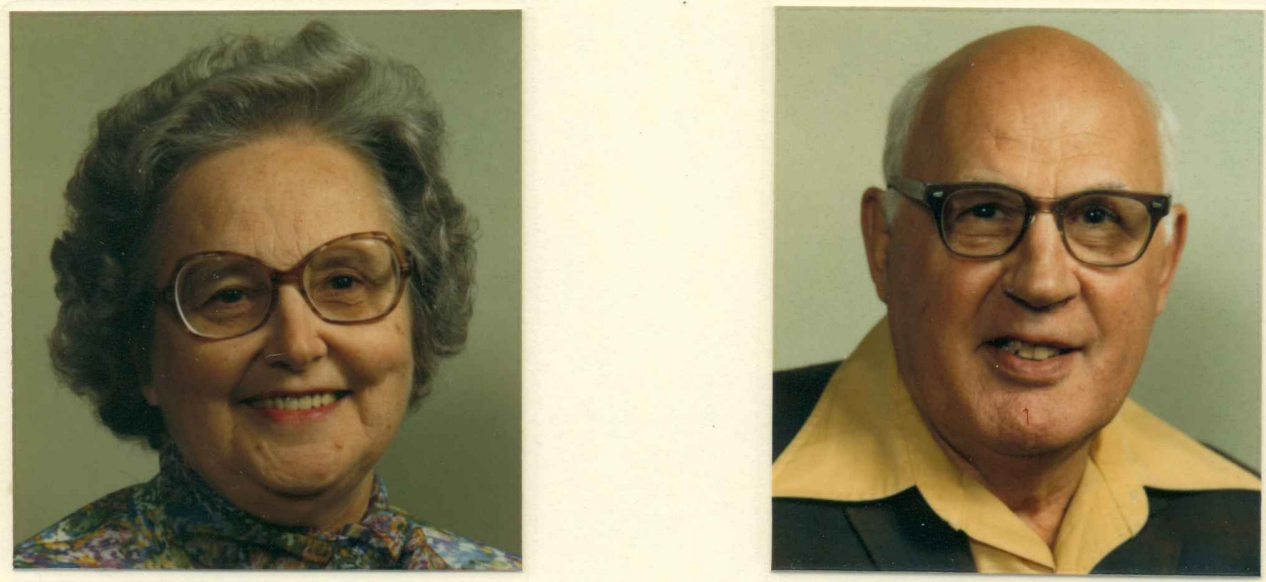

HIGH PHYSICAL ATTRACTIVENESS INTENSITY LEVEL
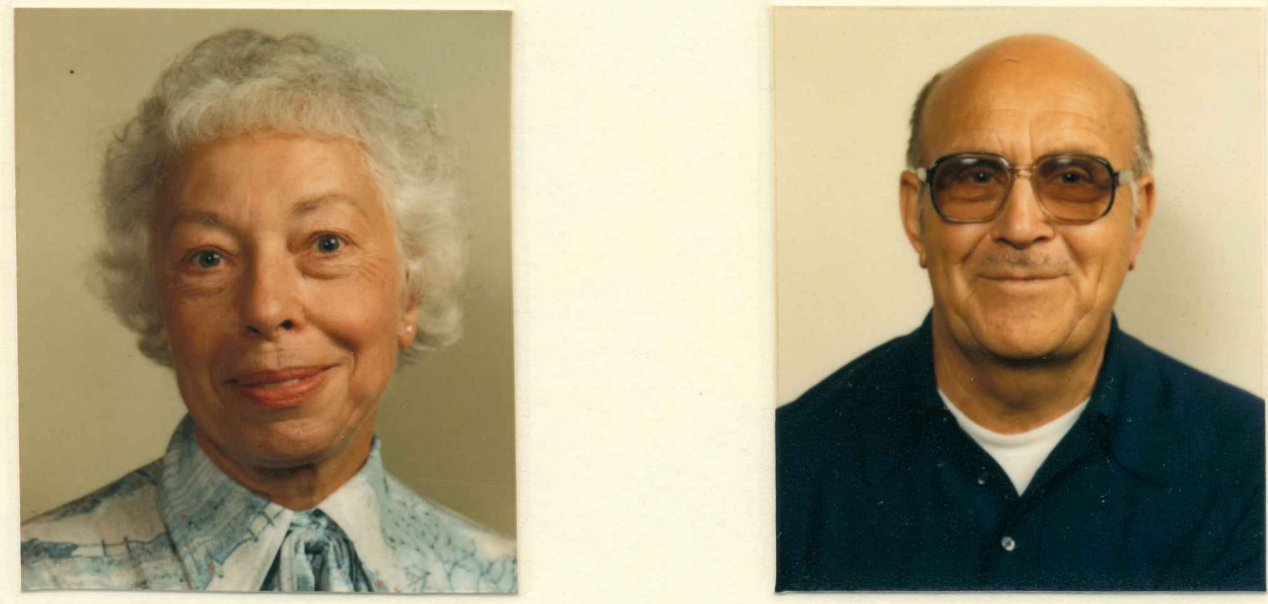

MEDIUM PHYSICAL ATTRACTIVENESS INTENSITY LEVEL
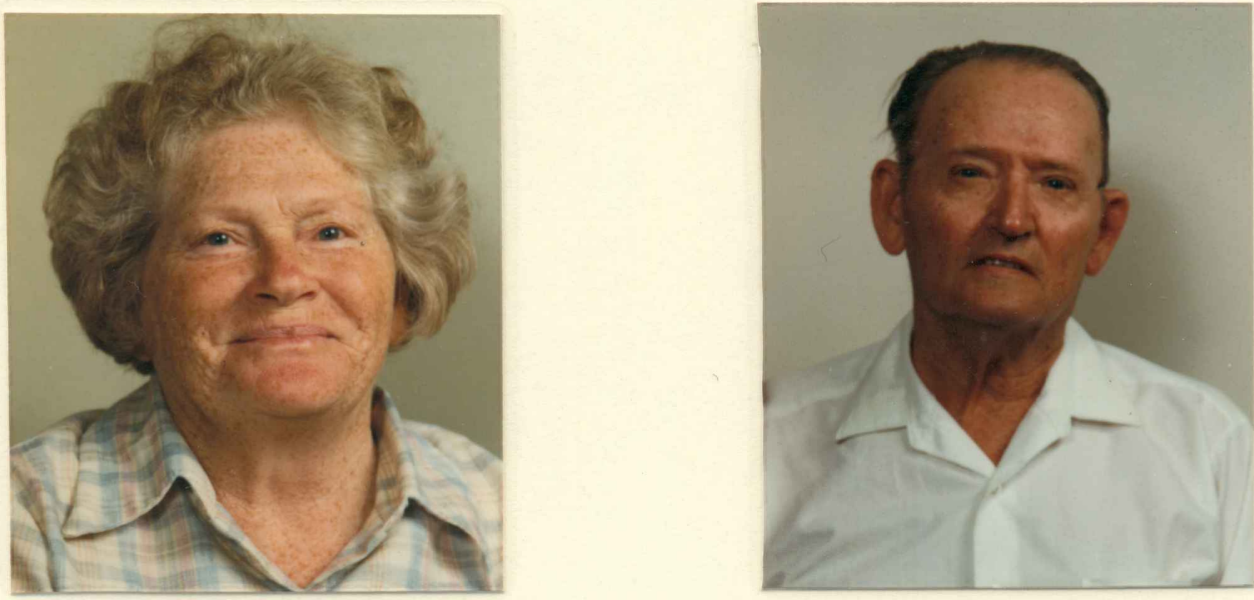

LOW PHYSICAL ATTRACTIVENESS INTENSITY LEVEL 
APPENDIX I

SUBJECTS 


\begin{tabular}{|c|c|c|c|}
\hline$\frac{\text { Photo Order } 1}{\text { Stimuti }}$ & $\begin{array}{l}\text { Intensity } \\
\text { Level s* }\end{array}$ & Age Group & Sex 1 \\
\hline $\mathbf{R}$ & Low & Young & M \\
\hline $\mathrm{F}$ & Medium & Young & $\mathrm{F}$ \\
\hline U & High & Middie-age & M \\
\hline I & Low & Middle-age & $F$ \\
\hline YY & Medium & $01 \mathrm{~d}$ & M \\
\hline KK & High & old & $\mathrm{F}$ \\
\hline $\mathrm{J}$ & Medium & Young & $M$ \\
\hline$M$ & High & Young & $\mathrm{F}$ \\
\hline $\mathrm{FF}$ & Low & Middle-age & $M$ \\
\hline CC & Medium & Middle-age & $\mathrm{F}$ \\
\hline 00 & Low & old & M \\
\hline II & Low & old & $\mathrm{F}$ \\
\hline$T$ & High & Young & $M$ \\
\hline 0 & Low & Young & $\mathrm{F}$ \\
\hline$z$ & Medium & Middle-age & M \\
\hline $\mathrm{EE}$ & High & Middle-age & $\mathrm{F}$ \\
\hline PP & High & $01 d$ & M \\
\hline MM & Medium & ord & $\mathrm{F}$ \\
\hline
\end{tabular}

Number of observations (subjects rating stimuli within each photo order), $N=12$, Six males and six females.

Number of stimuli, $N=18$

*Each intensity level was assigned a numerical value of High (3), Medium (2), or Low (1) determined by the judged (refer to page 112).

Sex was indicated by letter $M$ indicating Male or $F$ indicating Female above.

Figure 12. Photo order 1: Physical Attractiveness Intensity Levels, Age Group, and Sex. 
Photo Order 2 Intensity

Stimuli 10 Level s*

J

$\mathrm{FF}$

CC

00

II

R

$F$

U

I

YY

KK

$T$

0

Z

EE

PP

MM
Medium

High

Low

Medium

Low

Low

Low

High

Low

Medium

High

High

Low

Medium

$\mathrm{High}$

High

Medium
Medium
Age Group $\quad$ Sex 1

Young

Young

Middle-age

Middle-age

old

old

Young

Young

Middle-age

Middle-age

old

old

Young

Young

Middle-age

Mi ddle-age

old

$01 d$
M

$F$

M

$\mathrm{F}$

M

$F$

M

$F$

M

$F$

M

$F$

M

$F$

M

$F$

$M$

$F$

Number of observations (subjects rating stimuli within each photo order), $N=12$, Six males and six females.

Number of stimuli, $N=18$

*Each intensity level was assigned a numerical value of High (3), Medium (2), or Low (1) determined by the judged (refer to page 112). $1_{\text {Sex }}$ was indicated by letter $M$ indicating Male or $F$ indicating Female above.

Figure 13. Photo order 2: Physical Attractiveness Intensity Levels, Age Group, and Sex. 


\begin{tabular}{|c|c|c|c|}
\hline$\frac{\text { Photo Order } 3}{\text { Stimuli }}$ & $\begin{array}{l}\text { Intensity } \\
\text { Levels** }\end{array}$ & Age Group & Sex 1 \\
\hline$T$ & High & Young & $M$ \\
\hline 0 & Low & Young & $\mathrm{F}$ \\
\hline$z$ & Medium & Middle-age & M \\
\hline $\mathrm{EE}$ & $\mathrm{High}$ & Middle-age & $F$ \\
\hline $\overrightarrow{P P}$ & High & $01 \mathrm{~d}$ & $M$ \\
\hline MM & Medium & $01 d$ & $F$ \\
\hline $\mathrm{R}$ & Low & Young & M \\
\hline $\mathrm{F}$ & Medi um & Young & $\mathrm{F}$ \\
\hline U & High & Mi ddle-age & M \\
\hline I & Low & Middle-age & $F$ \\
\hline YY & Medium & $01 \mathrm{~d}$ & M \\
\hline KK & High & $01 \mathrm{~d}$ & $F$ \\
\hline $\mathrm{J}$ & Medium & Young & M \\
\hline M & $\mathrm{High}$ & Young & $F$ \\
\hline FF & Low & Middle-age & M \\
\hline CC & Medium & Middle-age & $F$ \\
\hline 00 & Low & $01 \mathrm{~d}$ & M \\
\hline II & Low & $01 d$ & $F$ \\
\hline
\end{tabular}

Number of observations (subjects rating stimuli within each photo order), $\mathrm{N}=12$, Six males and six females.

Number of stimul $i, \mathrm{~N}=18$

*Each intensity level was assigned a numerical value of High (3), Medium (2), or Low (1) determined by the judged (refer to page 112).

1 Sex was indicated by letter $M$ indicating Male or $F$ indicating Female above.

Levels, Age Group, and Sex. 3 : Physical Attractiveness Intensity 


\begin{tabular}{|c|c|c|c|}
\hline $\begin{array}{l}\text { Photo Order } 4 \\
\text { Stimuli }\end{array}$ & $\begin{array}{l}\text { Intensity } \\
\text { Levels } s^{\star}\end{array}$ & Age Group & Sex 1 \\
\hline $\mathbf{R}$ & Low & Young & M \\
\hline $\mathrm{F}$ & Medium & Young & $\mathrm{F}$ \\
\hline U & High & Middle-age & M \\
\hline I & Low & Middle-age & $\mathrm{F}$ \\
\hline$Y Y$ & Medium & $01 \mathrm{~d}$ & M \\
\hline KK & High & $01 \mathrm{~d}$ & $\mathrm{~F}$ \\
\hline$T$ & High & Young & $M$ \\
\hline 0 & Low & Young & $\mathrm{F}$ \\
\hline$z$ & Medi um & Middle-age & M \\
\hline EE & High & Middle-age & $\mathrm{F}$ \\
\hline $\mathrm{PP}$ & High & $01 \mathrm{~d}$ & M \\
\hline MM & Medium & $01 \mathrm{~d}$ & $\mathrm{~F}$ \\
\hline $\mathrm{J}$ & Medi um & Young & $M$ \\
\hline$M$ & $\mathrm{High}$ & Young & $\mathrm{F}$ \\
\hline $\mathrm{FF}$ & Low & Middle-age & M \\
\hline CC & Medium & Middle-age & $\mathrm{F}$ \\
\hline 00 & Low & $01 d$ & M \\
\hline II & Low & $01 \mathrm{~d}$ & $\mathrm{~F}$ \\
\hline
\end{tabular}

Number of observations (subjects rating stimuli within each photo order), $N=12$, Six males and six females.

Number of stimuli, $N=18$

*Each intensity level was assigned a numerical value of High (3), Medium (2), or Low (1) determined by the judged (refer to page 112). $1_{\text {Sex }}$ was indicated by letter $M$ indicating Male or $F$ indicating Female above.

Figure 15. Photo order 4: Physical Attractiveness Intensity Leve1s, Age Group, and Sex. 


\begin{tabular}{|c|c|c|c|}
\hline $\begin{array}{l}\text { Photo Order } 5 \\
\text { Stimuti }\end{array}$ & $\begin{array}{l}\text { Intensity } \\
\text { Level s* }\end{array}$ & Age Group & Sex 1 \\
\hline $\mathrm{J}$ & Medium & Young & M \\
\hline$M$ & $\mathrm{High}$ & Young & $F$ \\
\hline $\mathrm{FF}$ & Low & Middie-age & $M$ \\
\hline CC & Medium & Middle-age & $F$ \\
\hline 00 & Low & $07 \mathrm{~d}$ & M \\
\hline II & Low & $01 \mathrm{~d}$ & $\mathrm{~F}$ \\
\hline$T$ & High & Young & M \\
\hline 0 & Low & Young & $\mathrm{F}$ \\
\hline Z & Medium & Middle-age & M \\
\hline$\overline{E E}$ & High & Middle-age & $F$ \\
\hline $\mathrm{PP}$ & High & $01 \mathrm{~d}$ & $M$ \\
\hline MM & Medium & $01 d$ & $F$ \\
\hline$R$ & Low & Young & M \\
\hline $\mathrm{F}$ & Medium & Young & $\mathrm{F}$ \\
\hline$U$ & High & Middle-age & M \\
\hline I & Low & Middle-age & $F$ \\
\hline YY & Medium & old & $M$ \\
\hline KK & High & $01 d$ & $\mathrm{~F}$ \\
\hline
\end{tabular}

Number of observations (subjects rating stimuli within each photo order), $N=12$, Six males and six females.

Number of stimuli, $N=18$

*Each intensity level was assigned a numerical value of High (3), Medium (2), or Low (1) determined by the judged (refer to page 112).

Sex was indicated by letter $M$ indicating Male or $F$ indicating Female above.

Figure 16. Photo order 5: Physical Attractiveness Intensity Levels, Age Group, and Sex. 


\begin{tabular}{|c|c|c|c|}
\hline $\begin{array}{l}\text { Photo Order } 6 \\
\text { Stimuli } 10\end{array}$ & $\begin{array}{l}\text { Intensity } \\
\text { Level s* }\end{array}$ & Age Group & $\operatorname{Se} x^{1}$ \\
\hline$T$ & High & Young & $M$ \\
\hline 0 & Low & Young & $F$ \\
\hline$z$ & Medium & Middle-age & $M$ \\
\hline EE & High & Middle-age & $F$ \\
\hline PP & High & old & $M$ \\
\hline MM & Medium & old & $\mathrm{F}$ \\
\hline$J$ & Medium & Young & $M$ \\
\hline$M$ & High & Young & $F$ \\
\hline FF & Low & Middle-age & $M$ \\
\hline $\mathrm{CC}$ & Medi um & Mi ddle-age & $F$ \\
\hline 00 & Low & old & $M$ \\
\hline II & Low & $01 \mathrm{~d}$ & $F$ \\
\hline $\mathbf{R}$ & Low & Young & $M$ \\
\hline $\mathbf{F}$ & Medi um & Young & $\mathrm{F}$ \\
\hline U & High & Middle-age & $M$ \\
\hline $\mathrm{I}$ & Low & Middle-age & $\mathrm{F}$ \\
\hline YY & Medi um & $01 \mathrm{~d}$ & $M$ \\
\hline KK & High & $01 d$ & $F$ \\
\hline
\end{tabular}

Number of observations (subjects rating stimuli within each photo order), $N=12$, Six males and six females.

Number of stimuli, $\mathrm{N}=18$

*Each intensity level was assigned a numerical value of High (3), Medium (2), or Low (1) determined by the judged (refer to page 112).

$1_{\text {Sex }}$ was indicated by letter $M$ indicating Male or $F$ indicating Female above.

Figure 17. Photo order 6: Physical Attractiveness Intensity Levels, Age Group, and Sex. 